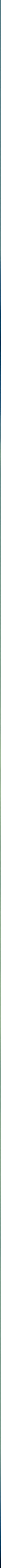

Multi-level Reconfigurable Self-organization in

Overlay Services

Evangelos Pournaras 



\title{
Multi-level Reconfigurable Self-organization in Overlay Services
}

\author{
Evangelos Pournaras
}

Delft University of Technology 



\title{
Multi-level Reconfigurable Self-organization in Overlay Services
}

\section{Proefschrift}

\begin{abstract}
ter verkrijging van de graad van doctor aan de Technische Universiteit Delft, op gezag van de Rector Magnificus prof. ir. K.C.A.M. Luyben, voorzitter van het College voor Promoties, in het openbaar te verdedigen op dinsdag 26 maart 2013 om 10:00 uur door
\end{abstract}

Evangelos POURNARAS

Master of Science in Internet Computing geboren te Athene, Griekenland. 
Dit proefschrift is goedgekeurd door de promotor:

Prof. dr. F.M.T. Brazier

Copromotor: Dr. M.E. Warnier

Samenstelling promotiecommissie:

Rector Magnificus, voorzitter

Prof. dr. F.M.T. Brazier, Technische Universiteit Delft, promotor

Dr. M.E. Warnier, Technische Universiteit Delft, copromotor

Prof. dr. R.E. Kooij, Technische Universiteit Delft

Prof. dr. G.J.M. Smit, Universiteit Twente

Prof. dr. I. Stavrakakis, National and Kapodistrian University of Athens

Prof. dr. M.N. Huhns, University of South Carolina

Dr. R. Ambrosio, $\quad$ IBM Thomas J. Watson Research Center

Prof. dr. Y. Tan, Technische Universiteit Delft, reservelid

Cover concept by Evangelos Pournaras:

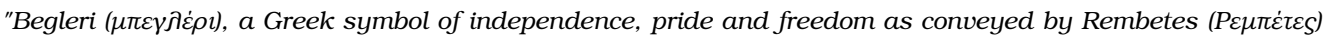
and their culture. In the cover of this thesis, begleri is introduced as a metaphor of overlay networks by lying on top of a tree topology built by AETOS, as shown in Table 4.2. Mutual reflections between the begleri and the topology of AETOS imply the interactions and interdependences in overlay networks."

Cover design by Hanike Studio: www . hanike.nI

Typeset with $\mathrm{IAT}_{\mathrm{E}} \mathrm{X} 2_{\mathcal{\varepsilon}}$

Layout by Evangelos Pournaras

Kerkis (C) Department of Mathematics, University of the Aegean

Printed by Uitgeverij BOXPress: www.proefschriftmaken.nI

Published by Uitgeverij BOXPress, 's-Hertogenbosch

This research was funded by NLnet: www. nl net. nl

Copyright (C) 2013 by Evangelos Pournaras

E-mail: mail@evangelospournaras.com

www. evangelospournaras.com

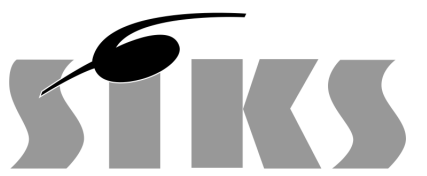

SIKS Dissertation Series No. 2013-11

The research reported in this thesis has been carried out under the auspices of SIKS, the Dutch Research School for Information and Knowledge Systems.

All rights reserved. No part of the material protected by this copyright notice may be reproduced or utilized in any form or by any means, electronic or mechanical, including photocopying, recording or by any information storage and retrieval system, without the prior permission of the author.

ISBN: 978-94-6186-098-9 
Your Life has brought me an aura of Sofia. Dedicated to You! 



\section{Preface}

The endeavor of scientific research sets out a traveling surge to a discovery path lying towards a far and hovering destination. The completion of this thesis has been such a traveling experience towards the "Ithaca" of Cavafy [1992]. As he wisely says, "hope for a long voyage full of adventure, full of knowledge". During this delightful journey, several people made the actual journey to matter the most. I would like to heartily thank them for this.

First and foremost, I would like to thank my promoter, Frances M.T. Brazier, for giving me the opportunity to join her group at VU University Amsterdam. I would also like to thank her for the encouragement and support when we moved to Delft University of Technology. Her words made me shape a more complete understanding about the world of academia. Frances provided me with new insights about how to communicate research. Through my interactions with her I learned how to ground my expectations, yet expand my ambitions.

Special thanks go to Martijn Warnier, my supervisor. Martijn has been an invaluable source of knowledge, inspiration and motivation. He has always been present in so many different ways whenever I needed him. My scientific writing would not improve to such an extent without his continuous feedback and tips. His encouragement to improve various technical skills has proven to be prophetic. Our brainstorming sessions made research so much fun. Without him, this thesis would not reach this quality. I am lucky and proud to be the first PhD student of Martijn.

It has been a pleasure to have several great colleagues at Delft University of Technology. One representative example is Kassidy Clark, my paranymph. I enjoyed so much accompanying the hard moments of our PhD studies with the cholesterol of our BBQs. Eventually, Kassidy knows now why you always serve lamp chops with lemon! I also thank him for his enthusiasm to provide feedback on my work. I enjoy discussions with Yakup Koç as much as I enjoy Greek coffee! I am so glad we are currently working on the same project. Moments in the gray Netherlands became more colorful with Jordan Janeiro and his Brazilian caipirinhas. Special thanks to Michel Oey for his technical support and advice throughout my PhD studies. Furthermore, it was a pleasure to spend moments in and out of work with Çağri Tekinay, Michele Fumarola, Shalini Kurapati, Yilin Huang, Selin Ebeci, Farideh Heidari and Jan-Paul van Staalduinen. I shared the same pleasure having interesting discussions with Reza Haydarlou, Pieter de Vries, Jos Vrancken, Mohsen Davarynejad, Caroline Nevejan, Thieme Hennis, Rick van Krevelen, Rens Kortmann, Joseph Barjis, Elizabeth Ogston and Sander van Splunter. I would like to truly thank all these people and many more from the System Engineering section for creating a pleasant working atmosphere. Finally, I would like to thank Robert Kooij, Piet Van Mieghem and all my other new colleagues in the Network Architecture 
and Services group for their warm welcoming and support. One of them, Martijn van Egdom, provided me with comments on Chapter 6 and translated the summary of this thesis in Dutch.

Looking back at VU University of Amsterdam, I would like to thank Rienier Timmer for his technical support, Rogier van het Schip for always switching to English and Thomas Quillinan for his initial support to set myself up in the Netherlands. I am sure it was not how he imagined this beginning for me, however, I truly did not imagine as well the large impact of Greek spirit on him! The coffee discussion with Spyros Voulgaris in Zurich was so encouraging for me to finally come at VU University Amsterdam. Furthermore, the voice of Andrew S. Tanenbaum has been enlightening in various fundamental issues of computer science. Finally, I would like to thank Maarten van Steen for the very few but very constructive discussions we had. His work and view on large-scale distributed systems have been inspiring and influential.

I furthermore would like to thank all members of my PhD committee for their fruitful comments and discussions. Credits also go to Wojciech Galuba and the development team of the Protopeer toolkit. Wojciech's technical support and responsiveness was crucial to complete the experimental work of this thesis. Veronique Eijkemans and her colleagues from Uitgeverij BOXPress have provided a highly professional technical support for printing and publishing this thesis. Hani Alers and Lucy Gunawan have been so patient to convey my continuously new ideas and desires in the cover of this thesis. Finally, I would also like to thank John Paravantis and George Lekakos for their invaluable advice throughout the time I am abroad.

It was a fortune to spend three incredible months as an intern at IBM Thomas J. Watson Research Center. I will never forget the times I spent with Mark Yao and Ron Ambrosio in their fascinating lab. I am especially proud that my work contributes to the Pacific Northwest Smart Grid Demonstration Project, thanks to Mark and Ron for giving me the opportunity to work in such a world-leading and cutting-edge research effort. I would also like to thank Dario Gil, my manager in IBM, for his support during my internship. Special thanks go to Brian Gaucher and Jeff Kephart who brought me in touch with Mark and Ron. Finally, I would like to thank my flatmate in New York, Bhooshan Ganu, for our unforgettable moments in crazy Rod's house!

Another fortune has been the traveling opportunities during my $\mathrm{PhD}$ studies. My travels to Portugal, Cyprus, Great Britain, Italy, Austria, Switzerland, Canada, the USA and especially the ones to Japan and South Korea have been an intellectual catalyst. I would like to send greetings to Haruka Fujisaki, Mamoru Nagasaki, Elise Bonzon and many other people all over the world! I am so glad to be in touch with you.

My close connection with several other people made expatriate life so much easier. I cannot underestimate the 'weight' of the undutchable George Portokalidis since I met him in Amsterdam. George was so patient listening me talking non-stop about Dutch girls during our legendary moments in 'our' whiskey bar. I have enjoyed the awesome hospitality and cooking skills of George in Amsterdam and New York, an integral part of his special Greek spirit. Time with Maria Tsimpiri looses its sense. I miss her so much! Interesting discussions with the Fred Hardig make it always easier to follow the German way of beer consumption. My first nights in Amsterdam with Anthi Schina and Michele Maio were enjoyably long. Moreover, there are some people back to Greece that make me always feel like I have never left from there. The remote support, encouragement and continuous communication with Sakis Mistakidis (my paranymph), Vasilis Manthopoulos and Maria Krikou have been a Greek shine in the darkness of the 
Netherlands. Dimitris Angelis, Charoula Manolioudaki and Yiannis Arapoglou make always their best to keep alive extraordinary memories from our BSc studies during my visits to Greece.

Life has also devastating moments during which you look for hope and someone to believe. Linda Pieper has definitely been my 'deus ex machina' (anó unxavńs Эعós). Linda has rotated my life. Her devotion, support, patience and care during the writing of this thesis and beyond are unique. Linda is always interested in hearing about my research, or Vasilis Tsitsanis, something that says a lot for her. I appreciate the great

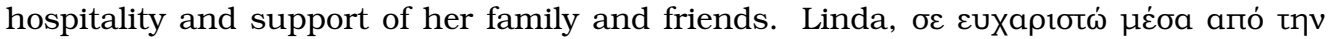

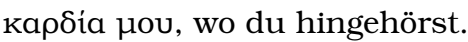

Last but not least, I would like to especially thank my family back in Greece, Stergios, Fanouria, Katerina $\supset$ and Elias. During all of the years I am abroad and despite the physical distance, my family has persistently been my vivid and unconditional supporter. Nothing can be compared with the truthful encouragement of my mother or the

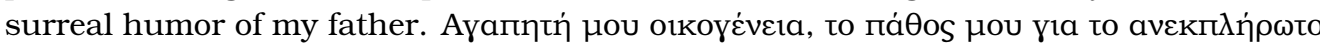

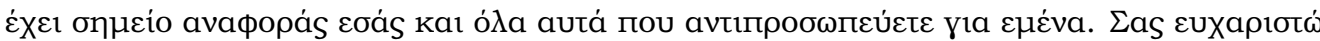

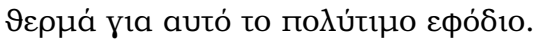

Evangelos Pournaras

March 2013

Delft 



\section{Summary}

The design and management of networked systems that are large-scale and decentralized is challenging. These systems are usually organized in virtual networks: the overlay networks. An overlay network lies at the application-level and on top of physical or other overlay networks. Overlay networks implement complex application and organizational functionality not supported by underlying network services. This integration and design approach results in low abstraction, modularity and reconfigurability of applications that are based on overlay networks. In contrast to this practice, this thesis introduces the conceptual architecture of ASMA, the Adaptive Self-organization in a Multi-level Architecture. ASMA is the main contribution of this thesis and is designed for building middleware systems of overlay networks that provide generic capabilities to different distributed applications: the overlay services.

The abstraction, modularity and reconfigurability of ASMA is achieved by its multilevel design approach. Three conceptually defined levels of overlay networks and their interactions provide discovery, structuring and coordination of system entities without a centralized management authority. The interactions between the three levels of ASMA form feedback loops that improve the quality of an overlay service incrementally. This thesis shows that a few lines of algorithmic expressions defined by ASMA are adequate to realize the complex system functionality of two introduced overlay services: (i) AETOS, the Adaptive Epidemic Tree Overlay Service and (ii) DIAS, the Dynamic Intelligent Aggregation Service. Both overlay services advance the state of the art by providing two generic application capabilities. AETOS builds and maintains overlay networks organized in tree topologies that meet different application criteria. DIAS computes different aggregation functions over a set of dynamically changing values distributed in an overlay network. Both overlay services of ASMA provide a proof-of-concept about their higher abstraction, modularity and reconfigurability at the cost of higher communication overhead compared to related work.

AETOS provides self-organization of tree topologies with the graph properties of degree-bounding, ordering, balancing and completeness. AETOS performs a gossip-based discovery of nodes in a network. These nodes, ranked according to application criteria, are clustered based on their proximity computed by their ranking distance. Clustering of nodes as candidate parents and children provides a more cost-effective search space compared to random searching. Bidirectional links are negotiated and established with these parents and children based on 'request', 'acknowledgment', 'rejection' and 'removal' interactions. Different tree topologies can be self-organized by adopting adaptation strategies that hide complex clustering and selection configurations. Experimental evaluation illustrates the performance trade-offs and reconfigurability of AETOS in various experimental settings. This evaluation concludes that AETOS is a generic and 
flexible overlay service for the self-organization of tree topologies.

DIAS makes aggregates, such as AVERAGE, SUMMATION, MAXIMUM, etc., locally available in every node of an overlay network. In contrast to other related methodologies, aggregation in DIAS is function-independent, routing-independent and dynamic as aggregates are adapted if distributed input values change during runtime. DIAS achieves this abstraction and flexibility by introducing the concept of aggregation memberships. An aggregation membership provides historic information about a computed aggregation value by indicating if this value is new, outdated or duplicate. This distinction guarantees accurate computation of aggregates. It also provides two adaptation strategies based on which new or outdated aggregation values may be preferred in computations of aggregates. An explicit storage of aggregation memberships is not a scalable and decentralized aggregation approach. Nevertheless, DIAS stores aggregation memberships in probabilistic data structures: the bloom filters. A bloom filter provides large space savings at the cost of false positives. A distributed consistency mechanism is introduced to detect false positives and, therefore, prevent inaccuracies in the computations of aggregates. Experimental evaluation confirms the high accuracy of DIAS under different experimental settings and performance trade-offs.

The applicability of AETOS and DIAS is studied in the domain of the Smart Power Grid. More specifically, two decentralized demand-side energy management mechanisms are introduced based on these overlay services: (i) EPOS, the Energy Plan Overlay Selfstabilization and (ii) ALMA, the Adaptive Load Adjustment by Aggregation. EPOS and ALMA are the contributions of this thesis in the application domain of the Smart Power Grid.

EPOS coordinates the energy consumption of a large number of thermostatically controlled devices such as water heaters, refrigerators etc., to achieve the global system objectives. More specifically, EPOS performs self-stabilization by eliminating the oscillations and power peaks in the total energy consumption if and when it is required. Thermostatic devices are controlled by communicating software agents that generate, select and execute operational plans for their devices without direct involvement and impact on consumers. EPOS achieves energy self-stabilization by using AETOS to selforganize agents in a tree overlay network within which they perform a decentralized aggregation and coordinated decision-making of their local energy consumption. Experimental evaluation using synthetic data shows the high energy stabilization achieved in various experimental settings.

ALMA complements EPOS under extreme conditions in which the Smart Power Grid requires an actual decrease or increase in the power demand due to failures or excessive micro-generation. ALMA achieves adjustments of aggregate energy consumption with possible demand options of local energy consumption, representing a wide range of comfort and economy levels, that can be pre-defined and dynamically selected by incentivized consumers. Aggregate information about power demand can be made locally available to consumers by the DIAS overlay service. The feasibility of ALMA is evaluated analytically using data from an operational Smart Power Grid: the Olympic Peninsula Smart Grid Demonstration Project.

In conclusion, this thesis indicates that introducing decentralized computing systems in an information era expanding to new critical application domains, such as the Smart Power Grid, is a promising endeavor towards more sustainable development and a resource-based economy in future societies. 


\section{Contents}

Preface $\quad$ vii

Summary $\quad$ xi

1 Introduction 1

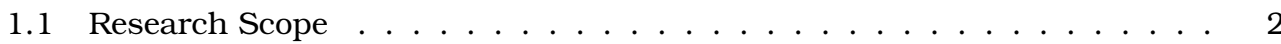

1.2 Research Objectives . . . . . . . . . . . . . . . . . 3

1.2.1 Conceptual gap . . . . . . . . . . . . . . . 3

1.2.2 Prototyping and performance evaluation . . . . . . . . . . 4

1.2 .3 Domain applicability ................ . . 4

1.3 Research Approach . . . . . . . . . . . . . . . . . . . . . 4

1.3.1 Research philosophy ................. 4

1.3.2 Research strategy . . . . . . . . . . . . . . . 5

1.3.3 Research instruments . . . . . . . . . . . . . 6

1.4 Contributions . . . . . . . . . . . . . . . . . . . 6

1.5 Thesis Outline . . . . . . . . . . . . . . . . 7

2 Overlay Networks and Services: Research Positioning 9

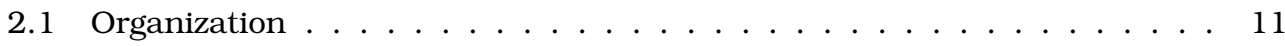

2.1.1 Structured overlay networks . . . . . . . . . . . . . . 12

2.1.2 Unstructured overlay networks . . . . . . . . . . . . . . 13

2.1.3 Comparison ..................... . . 14

2.2 Multiple Overlay Networks . . . . . . . . . . . . . . . . 16

2.3 Main Concepts and Positioning . . . . . . . . . . . . . . . 17

2.4 Prototyping Overlay Services . . . . . . . . . . . . . . . . . . 19

2.4.1 Protopeer . . . . . . . . . . . . . . . . 19

2.4.2 AgentScape and its simulation framework . . . . . . . . . . 20

2.5 Discussion and Future Work . . . . . . . . . . . . . . . . 22

2.5.1 The purist vs pluralist argument ... . . . . . . . . 23

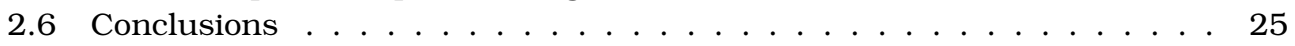

3 Adaptive Self-organization in a Multi-level Architecture $\quad 27$

3.1 Architecture Overview . . . . . . . . . . . . . . . . 27

3.1.1 The architectural levels . . . . . . . . . . . . . . . . 30

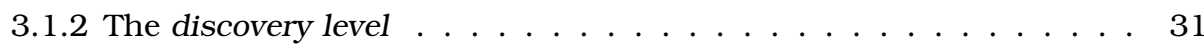

3.1 .3 The structuring level . . . . . . . . . . . . . . . . . . . . . . . . . . . . . . 33

3.1.4 The coordination level . . . . . . . . . . . . . . 36 
3.2 Architecture Coupling with Applications . . . . . . . . . . . . . . . . 38

3.3 Architectural Realization . . . . . . . . . . . . . . . . . . . . . 39

3.4 Comparison with Related Work . . . . . . . . . . . . . . . . . . 39

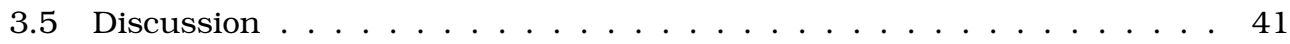

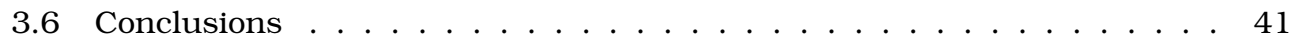

4 The Adaptive Epidemic Tree Overlay Service $\quad 43$

4.1 Problem Description . . . . . . . . . . . . . . . . . . 44

4.1.1 Graph properties and applications . . . . . . . . . . . . . 45

4.1 .2 Organizational goal . . . . . . . . . . . . . . . . . . . . . . . . . . . . . . . . . .

4.2 System Overview . . . . . . . . . . . . . . . . . . 51

4.3 Dissemination and Collection . . . . . . . . . . . . . . . 52

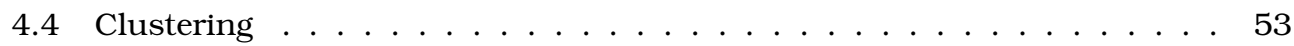

4.4.1 The input of the proximity view ... . . . . . . . . 54

4.4 .2 Adaptations . . . . . . . . . . . . . . . . . 55

4.5 Building and Maintenance . . . . . . . . . . . . . . . 57

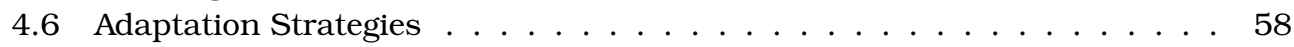

4.7 Experimental Evaluation . . . . . . . . . . . . . . . 6 60

4.7.1 Evaluation outline . . . . . . . . . . . . . . . . . . . . . . . . . . . . . 62

4.7.2 Adaptation strategies . . . . . . . . . . . . . . . . . . . . . . . . . . 63

4.7.3 The effect of adaptations . . . . . . . . . . . . . . . . 67

4.7.4 Dynamic adoption of the adaptation strategies . . . . . . . . . . 67

4.7.5 Reseting adaptations . . . . . . . . . . . . . . . 70

4.7.6 Tree topologies with different graph properties . . . . . . . . . 71

4.7.7 Adjusting the periodical executions . . . . . . . . . . . . 71

4.7.8 The effect of T-MAN . . . . . . . . . . . . . . . . . . . . 73

4.8 Evaluation Summary . . . . . . . . . . . . . . . . . . 73

4.9 Comparison with Related Work . . . . . . . . . . . . . . . 73

4.10 Discussion and Future Work . . . . . . . . . . . . . . . 77

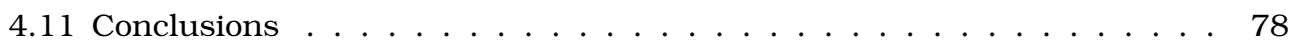

5 The Dynamic Intelligent Aggregation Service $\quad 79$

5.1 Problem Description . . . . . . . . . . . . . . . 81

5.2 System Overview . . . . . . . . . . . . . . . . . . . . . . . . . . . . . . . . . 82

5.3 Modeling of Dynamics . . . . . . . . . . . . . . . . . . 84

5.4 Dissemination and Collection . . . . . . . . . . . . . . . 85

5.5 Consistent Aggregation Sessions . . . . . . . . . . . . . . . 85

5.5.1 Aggregation memberships . . . . . . . . . . . . . 86

5.5 .2 Classification . . . . . . . . . . . . . . . . . 88

5.5 .3 Adaptation strategies . . . . . . . . . . . . . . . . . . . . . . . . . . . . 88

5.5 .4 Aggregation session . . . . . . . . . . . . . . . . . 89

5.6 Computation of Aggregates . . . . . . . . . . . . . . . . . 91

5.7 Realization based on Bloom Filters . . . . . . . . . . . . . . . . 93

5.7.1 The mutual membership check . . . . . . . . . . . . . . . 94

5.7 .2 First level check . . . . . . . . . . . . . . . . . . . 96

5.7 .3 Second level check . . . . . . . . . . . . . . . . . . . . 97

5.8 Experimental Evaluation . . . . . . . . . . . . . . . . 98

5.8 .1 Evaluation outline . . . . . . . . . . . . . . 101 
5.8 .2 Adaptation strategies . . . . . . . . . . . . . . . 101

5.8 .3 Bloom filter aggregation memberships . . . . . . . . . . . . . . 104

5.8 .4 Adjusting the size of the aggregation pool . . . . . . . . . . . 106

5.8.5 Adjusting the size of the aggregator classes . . . . . . . . . . . . . 107

5.8.6 Adjusting the number of aggregator samples . . . . . . . . . . . . . 107

5.8.7 Adjusting the number of aggregation sessions . . . . . . . . . . . 107

5.8 .8 Adjusting the periodical executions . . . . . . . . . . . . . 108

5.9 Evaluation Summary . . . . . . . . . . . . . . . . 108

5.10 Comparison with Related Work . . . . . . . . . . . . . . . . 109

5.11 Discussion and Future Work . . . . . . . . . . . . . . . 111

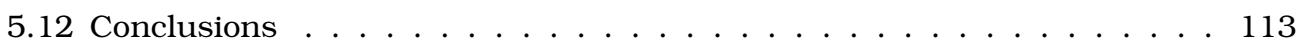

6 Demand-side Energy Management using Overlay Services 115

6.1 Demand-side Energy Management . . . . . . . . . . . . . . . 116

6.1 .1 Research background . . . . . . . . . . . . . 116

6.1 .2 Research focus . . . . . . . . . . . . . . . . 119

6.2 Overlay Services for Energy Management . . . . . . . . . . . . . . 119

6.3 Energy Plan Overlay Self-stabilization . . . . . . . . . . . . . . 120

6.3.1 Coordination of energy consumption . . . . . . . . . . . . 123

6.3.2 Using the AETOS overlay service . . . . . . . . . . . . . . 127

6.3 .3 Experimental settings . . . . . . . . . . . . . . . 128

6.3 .4 Evaluation results . . . . . . . . . . . . . . . . 130

6.3 .5 Evaluation summary . . . . . . . . . . . . . . . . . . . . . . . . . . . . . 134

6.4 Adaptive Load Management by Aggregation . . . . . . . . . . . . . . . . . 135

6.4.1 The Olympic Peninsula Project . . . . . . . . . . . . . . . 137

6.4 .2 Validation approach . . . . . . . . . . . . . . . 138

6.4 .3 Analytical results . . . . . . . . . . . . . . . . 140

6.4 .4 Interpretation of results . . . . . . . . . . . . . . . . 142

6.5 Comparison with Related Work . . . . . . . . . . . . . . . . . . 143

6.6 Discussion and Future Work . . . . . . . . . . . . . . . . . . . 144

6.7 Conclusions ........................ 145

7 Conclusions and Future Work 147

7.1 Meeting Research Objectives . . . . . . . . . . . . . . . . . . . . 148

7.2 Open Issues and Future work . . . . . . . . . . . . . . . . . . . 149

$\begin{array}{lr}\text { Bibliography } & 167\end{array}$

$\begin{array}{ll}\text { A Supplemental Material of Chapter } 4 & 169\end{array}$

B Supplemental Material of Chapter $5 \quad 175$

$\begin{array}{ll}\text { C Supplemental Material of Chapter } 6 & 177\end{array}$

$\begin{array}{ll}\text { List of Symbols } & 179\end{array}$

$\begin{array}{ll}\text { List of Figures } & 185\end{array}$

$\begin{array}{lr}\text { List of Tables } & 187\end{array}$ 
List of Algorithms

Samenvatting (Dutch Summary)

SIKS Dissertation Series

Publications

204

Biography

205

xvi 


\section{Chapter 1

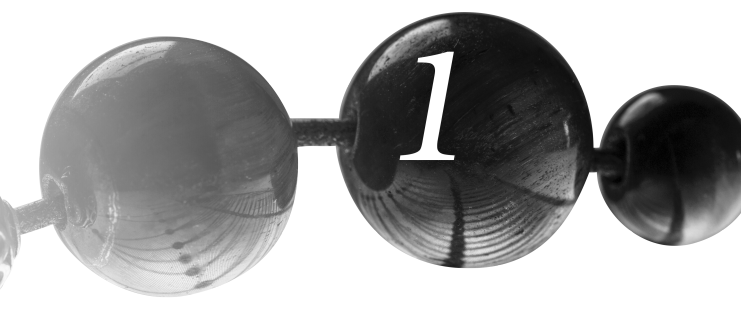

\section{Introduction}

"Every perfect traveler always creates the country where he travels."

Nikos Kazantzakis ${ }^{1}$

The design and management of networked systems that are large-scale and decentralized is challenging. These systems are complex and dynamic with a large number of autonomous software and hardware entities, whose interconnections, interactions and distributed operations frequently generate bursts of dynamically changing information. Such large-scale decentralized networked systems play a crucial role in emergent sociotechnical domains of application such as energy management [Ipakchi and Albuyeh, 2009].

Centralized control and management is not always a scalable and cost-effective option [Tanenbaum and Van Steen, 2007]. Within decentralized environments, meeting organizational and system objectives requires coordination of system entities and their interactions. This thesis studies self-organization and adaptation as a means for decentralized coordination. More specifically, the focus of this thesis is the design, prototyping and evaluation of generic and adaptive self-organizing systems for different applications with different requirements that operate within large-scale decentralized environments. Extending the scope, applicability and interoperability of a self-organizing system to a wide range of applications can be achieved with a conceptual architecture of higher abstraction and modularity based on services [Spohrer et al., 2007].

Cost-effective management of energy resources is the cornerstone for the sustainability and development of today's societies. Electrical power grids are critical sociotechnical systems that face new emergent challenges such as the variation of power demand [Strbac, 2008], the outdated infrastructure [Massoud Amin and Wollenberg, 2005], cyber-security threats [Khurana et al., 2010] and the introduction of new technologies, i.e., electrical vehicles [Clement-Nyns et al., 2010] and renewable energy resources [Brandstätt et al., 2011]. These challenges motivate the need for a more intelligent, adaptive, robust and cost-effective electrical power grid, the so called Smart Power Grid [Massoud Amin and Wollenberg, 2005, Ipakchi and Albuyeh, 2009]. Such a socio-technical system is more complex, heterogeneous, interconnected, decentralized

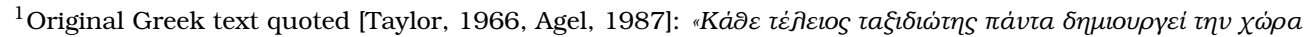

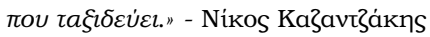


and information-oriented than the traditional electrical power grid. A representative example in this domain is demand-side energy management [Strbac, 2008, Albadi and El-Saadany, 2008, Palensky and Dietrich, 2011] that is the application focus of this thesis. Demand-side energy management is based on the principle of adapting power demand to existing available production. In contrast to this approach, production-side energy management requires costly investments and configurations for scaling up and down energy production. Demand-side energy management involves a large number of interactive entities that require coordination to match demand/supply, balance the load in the network and optimize the use of new emerging technologies such as electrical vehicles and renewable energy resources.

The introduction of generic and adaptive self-organization systems in the Smart Power Grid and beyond is a promising endeavor towards a more sustainable society that manages energy resources more cost-effectively. This thesis explores this endeavor.

\subsection{Research Scope}

A distributed system is a collection of independent entities that appears to its users to be a single coherent system [Tanenbaum and Van Steen, 2007]. These entities are usually autonomous with computational and networking capabilities, e.g., a computer, a mobile device or a cyber-physical system. A distributed system is decentralized if none of its entities have centralized control or authority over the system. Their operation may involve synergies between system entities and collective decisions. Large-scale decentralized systems consist of a large number, e.g., thousands or hundred of thousands, of remote networked entities that interoperate in parallel. These systems are complex as their size and operational interdependencies result in emergent phenomena that govern their behavior [Mogul, 2006]. Management and control of large-scale decentralized systems are challenges that this thesis addresses.

Examples of complex decentralized systems are overlay [Kurian and Sarac, 2010], peer-to-peer [Lua et al., 2005] and ad-hoc [Boulis et al., 2003] networks that can be part of socio-technical systems such as the Smart Power Grid [Birman, 2001, Fan et al., 2010, Hammerstrom et al., 2010, Erol-Kantarci et al., 2011]. Application examples include routing, search, aggregation, information dissemination and decision-making in domains such as multimedia multicasting [Birrer and Bustamante, 2007], distributed databases [González-Beltrán et al., 2008] and demand-side energy management [Palensky and Dietrich, 2011].

Overlay networks are subject of this thesis. They are a network virtualization [Anderson et al., 2005] of physical or other networks. Virtual (software) entities in an overlay network manage and control entities in an underlying network. The set of virtual interconnections (links) in an overlay network represents an organizational structure of the system or application logic. The concept of overlay networks is applicable to a wide range of large-scale decentralized systems and applications [Kurian and Sarac, 2010]. Information and interactions in a decentralized system can be modeled and organized in an overlay network. For example, a set of routing tables, a publish-subscribe system or an indexing structure of a distributed database contain information that can be managed within the virtual entities of an overlay network.

Operational functionality of a distributed application based on an overlay network often determines topological management and organization [Matei et al., 2002, Tan 
et al., 2005a]. This practice results in a complex integration of application logic with the management of its overlay network. In other words, such applications deal with topological and organizational complexity as a way to be dynamic, adaptive and capture information changes during their runtime. Improved performance is the main reason behind this practice, especially in cases of limited computational and storage resources on the Internet. This design approach results in low extensibility and customization of an application that requires one or more overlay networks to operate.

Yet, reusing and customizing services of large-scale decentralized systems organized in overlay networks is the challenge addressed in this thesis. Introducing a higher abstraction and modularity is a promising approach to meet this challenge as Grace et al. [2008], Joseph et al. [2006], Mao et al. [2008], Li et al. [2004], Rodriguez et al. [2004], Braynard et al. [2002] and Joita et al. [2005] identify. Abstraction concerns a hierarchical representation or generalization of system functionality. This functionality is made more domain-specific when implemented within an application domain, without redefining its principal concepts. Moreover, modularity is the process of subdividing a large complex system to smaller modules as a way to make the system more rigorous, reusable and customizable. Abstraction and modularity decrease the development and maintenance cost of a system, usually by sacrificing a level of performance [Balsamo et al., 2004]. Such performance trade-offs, made in order to introduce more generic application capabilities for overlay networks, are experimentally evaluated in this thesis.

This thesis studies the applicability of generic and large-scale decentralized systems organized in overlay networks in the energy domain. Distribution, decentralization and automation are potential requirements in several aspects of the Smart Power Grid, e.g., energy resources, market and home respectively. These requirements are some of the main features of large-scale decentralized computing systems. Therefore, this thesis views and studies the Smart Power Grid as a complex, networked, large-scale and decentralized socio-technical system.

\subsection{Research Objectives}

The research objectives of this thesis are summarized in the following research question:

Question. Can large-scale decentralized networked systems be designed to provide modular and reconfigurable overlay services?

The rest of this section summarizes the three aspects investigated to answer the above research question.

\subsubsection{Conceptual gap}

Generic services provide a broad scope of application capabilities that can be reused between different types or classes of applications. In the context of the aforementioned research question, such services can be designed by introducing an architecture with a higher abstraction and modularity concerning the (i) configuration, (ii) organization and (iii) optimization of overlay networks.

This thesis introduces a new conceptual architecture for the design of such services. The main modules of this architecture, their interactions and inter-relations are defined and studied in Chapter 3. 


\subsubsection{Prototyping and performance evaluation}

The abstraction and modularity of the aforementioned architecture is validated by prototyping two services that meet the requirements of a broad scope of applications.

The first service is the focus of Chapter 4. It addresses decentralized construction and maintenance of self-organized overlay networks in tree topologies. These topologies are organized with different graph properties to match different application criteria. The second service is the focus of Chapter 5. It addresses decentralized computation of a wide range of aggregation functions over dynamically changing values distributed in an overlay network.

Both services require different generic application capabilities: (i) The organization of tree topologies with different graph properties and (ii) the computation of multiple aggregation functions. This thesis experimentally studies performance trade-offs required to design and apply such generic services.

\subsubsection{Domain applicability}

The applicability of the two services discussed above is studied in the domain of the Smart Power Grid. Energy resources are distributed and interconnected to cyberphysical systems and networks of power grid infrastructures. These large and complex networks generate a large amount of management information that can be used to reason about system adaptation during runtime.

Overlay networks are a promising self-management solution for the future Smart Power Grid. They provide an intuitive design separation between (i) the underlying power grid infrastructure built by physical/engineering assets and (ii) a virtual communication network positioned above. An overlay network organizes and manages information required for the configurations of the underlying infrastructure.

This thesis specifically studies the feasibility of services based on overlay networks for demand-side energy management. Chapter 6 focuses on this domain. Two reasons motivate the introduction of overlay networks for demand-side energy management: (i) The virtualization of overlay network does not impose major infrastructural changes and (ii) consumers are increasingly networked on an lnternet-scale.

\subsection{Research Approach}

Meeting the research objectives of this thesis requires knowledge and significant understanding of various research areas. Computer networks, distributed systems, complex systems and networks, artificial intelligence, software and electrical engineering are some examples. This section illustrates the research approach to answer the research question of this thesis.

\subsubsection{Research philosophy}

The main research philosophy followed in this thesis is positivism. According to the philosophy of positivism [Creswell, 2008], scientific knowledge comes from an objective, observable and measurable reality from which collected data build an empirical evidence. The process of data collection and the findings coming from empirical evidence should be repeatable. In practice, positivists are reductionists in the sense that 
they decompose a studied problem to its simplest possible elements. This is a common practice in the research area of systems that are large, decentralized and complex.

A secondary research philosophy adopted is interpretivism. According to interpretivism [Creswell, 2008], scientific knowledge comes from a non-rigid reality influenced by its context, therefore, building different realities to generate knowledge is possible. The scientific understanding of interpretivists is hermeneutic and prejudiced as it stands between the studied events and the individuals, who concern both researchers and participants.

Part of Chapter 6 focuses on demand-side energy management using data concerning the power demand of consumers. These data are collected from a specific demonstration project of Smart Power Grid technologies [Hammerstrom, 2007]. Therefore, this demonstration project is the context in which part of the scientific knowledge in this thesis is grounded.

Note that a pluralistic research methodology supported by more than one research philosophy is an approach motivated among various research communities [Mingers, 2001, Crossan, 2003].

\subsubsection{Research strategy}

The research strategy followed in this thesis is design science, outlined in the seven guidelines of [Hevner et al., 2004].

The outcome of design science research is a purposeful and innovative artifact ${ }^{2}$ (guideline 1). In this thesis, the artifact is a conceptual architecture for building generic services for large-scale decentralized systems. The artifact is studied in the current reality and practice of an application domain, i.e., the energy domain in this thesis (guideline 2). The effectiveness of the artifact is rigorously shown using relevant and well-established methods (guideline 3) that provide verifiable contributions in the studied research area (guideline 4). Prototyping and evaluation of two artifact (architecture) realizations, meet these two guidelines in this thesis. Construction and evaluation of the designed artifact relies on the application of rigorous methods that entail mathematical formulations and appropriate performance metrics (guideline 5). Chapter 4 and 5 study services of large-scale decentralized systems based on such rigorous methods. The artifact is improved during the evaluation process by testing design alternatives that satisfy the research problem and objectives (guideline 6). Various performance trade-offs discussed throughout this thesis are a result of these design alternatives. Finally, the results of design science should be communicated in a broader research context as a means to build a cumulative knowledge and motivate future work (guideline 7). Chapter 6 and 7 discuss and envision the impact of this research in future energy systems and sustainable societies.

In contrast to the alternative paradigm of behavioral science that seeks to discover "what is true", design science pursues to create "what is effective". In design science, the building and applicability of a designed artifact generates scientific knowledge. Design science does not exclude behavioral aspects in the study of artifacts, as these aspects are the ones that confirm the feasibility of the artifact in a context. The same holds for this thesis. The conceptual architecture introduced is studied within the application

\footnotetext{
${ }^{2}$ Constructs, models, methods and instantiations are general examples of artifacts in design science that refer to concepts, symbols, abstractions, representations, algorithms, prototypes, frameworks etc.
} 
domain of energy management as a way to show the purposefulness and innovation of its realizations, the services.

\subsubsection{Research instruments}

The research of this thesis involves five types of research instruments [Sjoberg et al., 2007]: (i) literature review, (ii) experiment, (iii) evaluation, (iv) case study and (v) aggregate data analysis.

Literature review provides the background knowledge required to conduct research. It motivates the research question of this thesis and is used for the comparison of the designed services with related work.

Experiment is the tool used for testing the functionality of the services and their applicability in the energy domain. The empirical evidence that this thesis provides comes from controlled experiments performed in simulation and emulation software environments. Section 2.4 illustrates the main experimental software environments used. Chapter 4, 5 and 6 illustrate the experiments that support the claims of this thesis.

Evaluation follows each of the performed experiments. For example, performance trade-offs are evaluated to show the cost-effectiveness of the introduced services.

The applicability of the introduced services is studied in Chapter 6 using case studies in the energy domain. Both case studies represent scenarios of demand-side energy management.

Finally, aggregate data analysis follows each illustrated case study. Both synthetic and real data in the energy domain are used in the analyses.

\subsection{Contributions}

The main contribution of this thesis in a conceptual architecture for generic services of a broad application scope based on overlay networks (Chapter 3).

This contribution advances the state of the art in the research area of large-scale decentralized systems by introducing, in these systems, two novel generic capabilities as services:

1. Self-organization of different tree topologies of overlay networks for different application criteria (Chapter 4).

Overlay networks are optimized in different tree topologies by only using highlevel adaptation strategies within the same self-organization architecture. The combination of these strategies results in performance benefits compared to the outcome of each adaptation strategy independently.

2. Computation of different aggregation functions over a set of dynamically changing values distributed in an overlay network (Chapter 5).

Each entity of a large-scale decentralized system is aware of system-wide parameters computed by aggregation functions. Computations are routing-independent. High-level adaptation strategies manage accuracy trade-offs concerning the computation of aggregation functions, i.e., aggregating undiscovered values versus aggregating values that change. 
Furthermore, this thesis contributes to the current research efforts of moving the traditional electrical power infrastructures towards the Smart Power Grid [Massoud Amin and Wollenberg, 2005, Ipakchi and Albuyeh, 2009]. The role of demand-side energy management is crucial in this research effort [Palensky and Dietrich, 2011]. Two decentralized mechanisms are introduced based on the aforementioned services that achieve the following energy management goals (Chapter 6):

1. Self-stabilization of energy consumption without direct involvement of consumers.

Demand is shifted or spread more uniformly in time resulting in lower power peaks and oscillations.

2. Self-adjustment of energy consumption by incentivized consumers who choose between multiple demand options.

Demand is increased or decreased based on the availability of power supply.

Note that the contributions of this thesis bring two research areas closer to each other: (i) distributed computing and (ii) energy management: On the one hand, the applicability of services based on overlay networks is studied in the domain of demandside energy management. On the other hand, large-scale decentralization in demandside energy management is studied using services based on overlay networks.

\subsection{Thesis Outline}

This thesis is outlined in the seven chapters illustrated in Figure 1.1.

Chapter 2 positions the research of this thesis within related work on overlay networks and services of large-scale decentralized systems.

Chapter 3 illustrates the main introduced artifact, ASMA, the Adaptive Self-organization in a Multi-level Architecture. ASMA is the conceptual architecture that can be used to build generic services based on overlay networks.

Chapter 4 introduces AETOS, the Adaptive Epidemic Tree Overlay Service. AETOS is the first generic service realized by the ASMA architecture. It builds and maintains different tree topologies of self-organized overlay networks that match different application criteria.

Chapter 5 introduces DIAS, the Dynamic Intelligent Aggregation Service. DIAS is the second service realized by the ASMA architecture and is designed to compute different aggregation functions over a set of dynamically changing values distributed in an overlay network.

Chapter 6 studies the applicability of AETOS and DIAS in the energy domain. More specifically, two decentralized demand-side energy management mechanisms are introduced. Each mechanism uses AETOS and DIAS respectively. These mechanisms make possible large-scale and automated self-management of networked consumers in the Smart Power Grid.

The first demand-side energy management mechanism is EPOS, the Energy Plan Overlay Self-stabilization. EPOS stabilizes the total energy consumption originated within a network of consumers. This is achieved by controlling and coordinating the power demand of specific household devices that do not require significant involvement of consumers. 


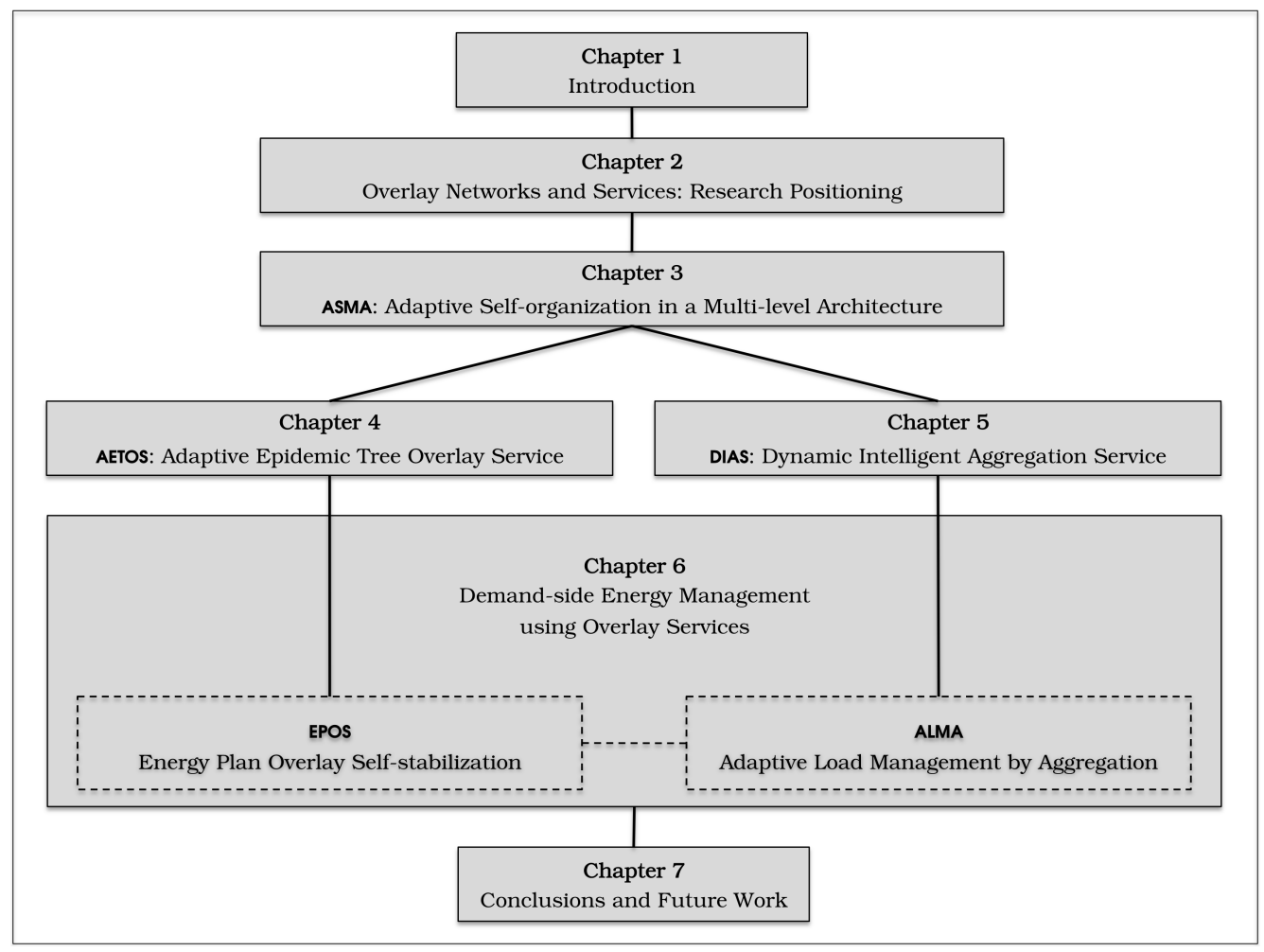

Figure 1.1: An outline of this thesis.

The second mechanism is ALMA, the Adaptive Load Management by Aggregation. ALMA adjusts the total energy consumption by involving incentivized consumers who choose between multiple demand options representing comfort and economy levels of their energy consumption.

Finally, Chapter 7 concludes this thesis by discussing the main findings and limitations. It also outlines the future research opportunities and work.

Note that lists with the symbols, figures, tables and algorithms are summarized at the end of this thesis. 


\section{Chapter 2

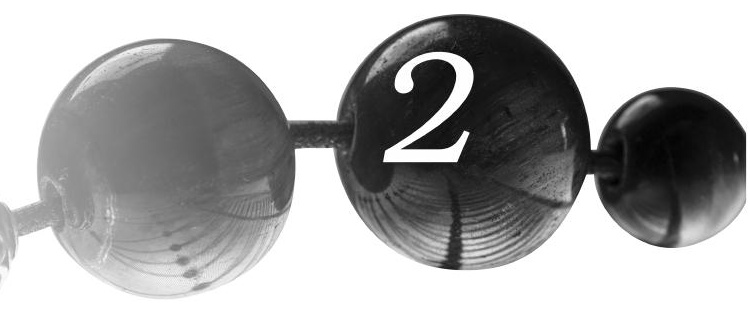

\section{Overlay Networks and Services: Research Positioning}

"The field needs a philosophical revolution in how developers use overlays, not a technical alteration in how they build them."

Anderson et al. [2005]

This chapter discusses network virtualization of overlay networks in large-scale dynamic decentralized systems that support different applications. This chapter shows that the organizational aspects of overlay networks is a critical influential factor to consider in the design of these systems.

The concept of network virtualization [Anderson et al., 2005, Chowdhury and Boutaba, 2009] provides network abstraction, transparency and interoperability to interconnected systems such as the Internet. An overlay network is a virtual network residing over a another network that is either an overlay or a physical network. It is often represented as a graph with links that interconnect nodes corresponding to virtual computational entities as explained in Section 2.3. The links of an overlay network are also virtual and may correspond to routing paths in a physical network. Note that overlay links can either be bidirectional or unidirectional. A bidirectional overlay link between two overlay nodes may represent a $\mathrm{TCP}^{1}$ connection. The unique network identifiers of physical hosts, such as the IP address and port number may define an overlay link. However, other application-related meta-information may also be associated with an overlay link.

Figure 2.1 illustrates the concept of overlay networks. Similar to other types of network virtualization, overlay networks are based on the following architectural principles discussed by Chowdhury and Boutaba [2009]: (i) coexistence, (ii) recursion (nesting) and (iii) inheritance. Coexistence refers to two or more independent overlay networks, usually one for each distributed application, positioned over a given physical network. In Figure 2.1, overlays (1)-(2) and (3)-(4) coexist. Recursion (nesting) refers to an overlay network that relies on information inherited from a second underlying overlay. Recursion defines a parent-child relationship between two overlay networks. The overlay

\footnotetext{
${ }^{1}$ Transmission Control Protocol [Tanenbaum and Van Steen, 2007]
} 
networks (1)-(3) and (2)-(4) in Figure 2.1 are nested and inherit organizational information such as overlay links or other application-related information managed by an overlay network.

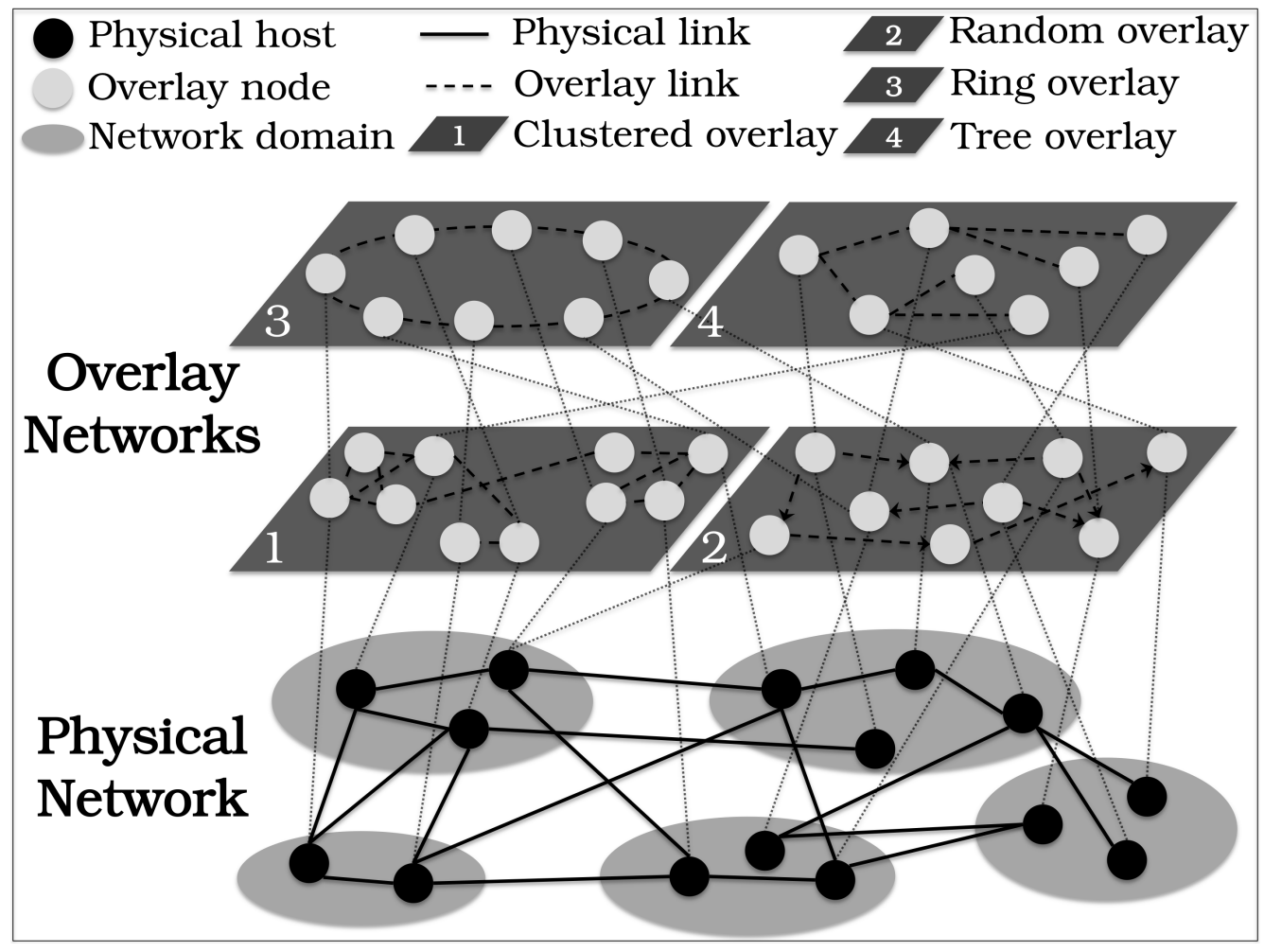

Figure 2.1: The concept of overlay networks as a network virtualization over physical and other overlay networks.

Clark et al. [2006] identify three main reasons that distributed applications adopt overlay networks: (i) heterogeneous user interests and application requirements, (ii) dynamic evolution of the Internet that entails incremental innovation adoption and (iii) potential conflicts between customers, service providers and policy-makers. Furthermore, these three reasons shape six classes of overlay networks: (i) Peer-to-peer, (ii) contentdelivery, (iii) routing, (iv) security, (v) experimental and (vi) other. Note that the Internet itself started as an overlay network on top of telecommunication networks [Clark et al., 2006, Chowdhury and Boutaba, 2009].

Overlay networks are not only used as a (i) deployment step for radical Internet architectural interventions but also as a (ii) design artifact for a wide range of large-scale dynamic decentralized systems. Distributed applications adopt overlay-based solutions to overcome the inflexibility and technical challenges of a physical network. For example, the Internet faces technical challenges such as the adoption of IPv6 [Colitti et al., 2010], the deployment of IP multicasting [Diot et al., 2000], the increased peer-to-peer traffic that Internet Service Providers (ISPs) experience [Chang et al., 2006] and the heterogeneity of the Internet of Things that ranges from small sensors to mainframe 
machines. Overlay networks provide application-level network virtualization as the means to handle underlying network complexity. Application-level multimedia multicasting [Tan et al., 2005a] is an example of such a widely adopted alternative to IP multicasting. Overlay networks support distributed applications [Birrer and Bustamante, 2007, González-Beltrán et al., 2008] with operations such as decentralized information lookup, discovery, routing, aggregation, dissemination, etc.

The choice of topological organization in an overlay network is related to the supported application capabilities and the performance or application requirements that need to be met. This chapter distinguishes two classes of topologies in overlay networks: structured and unstructured. This chapter shows that these two classes exhibit different topological properties that are able to meet different application requirements. Combining overlay networks, structured or unstructured ones, has been documented as an approach with potential collective performance advantages from both classes [Castro et al., 2005, Honghao et al., 2005]. The aforementioned architecture principle of nesting is an example that indicates a way of combining overlay networks. However, introducing generic architectures of multiple (nested) overlay networks, able to provide generic capabilities to applications of large-scale dynamic decentralized systems, is a research area that has not yet been fully explored. The research work of this thesis is positioned in exactly this research area.

This chapter is organized as follows: Section 2.1 illustrates and compares the two main organizational approaches for designing overlay networks and their applications: structured and unstructured. Sections 2.2 addresses architectures that combine multiple overlay networks. Section 2.3 introduces and positions the main concepts used in the rest of this thesis. Section 2.4 illustrates the prototyping tools used in this thesis for system design, development and evaluation. Section 2.5 discusses the purist vs. pluralist argument and other issues related to overlay networks and services. Finally, Section 2.6 concludes this chapter.

\subsection{Organization}

The organization of an overlay network is defined as the topological arrangement of its overlay links, nodes and their information. Organization often determines the functionality that an application performs, i.e., information routing, lookup, search, decisionmaking, dissemination, aggregation, etc. The topological (graph) properties of an overlay network influence the efficiency of applications. A wide range of self-organization mechanisms have been proposed that configure the organizational properties to enhance application performance.

This section reviews two main organizational classes of overlay networks and their applications: (i) structured and (ii) unstructured. The importance of these two classes is addressed in literature [Lua et al., 2005, Castro et al., 2005, Yang et al., 2006, Scholtes and Tessone, 2012]. This chapter reviews their generic application capabilities. Note that large-scale decentralized systems can also be designed with multiple structured and unstructured overlay networks as discussed in Section 2.2. 


\subsubsection{Structured overlay networks}

Structured systems of overlay networks are usually built by the mapping of system entities and their information, e.g., IP address or application-related information, to an identifier or proximity space [Aberer et al., 2005]. This space can be represented as a topological structure, e.g., ring or tree topology, of certain graph properties that may be related to available network resources or other application requirements and constraints.

A wide range of structured overlay networks are built on distributed hash tables (DHTs), i.e., CAN [Ratnasamy et al., 2001], Chord [Stoica et al., 2003], Tapestry [Zhao et al., 2004], Pastry [Rowstron and Druschel, 2001], Kademlia [Maymounkov and Mazières, 2002] and Viceroy [Malkhi et al., 2002]. Most of these DHTs are based on an abstract keyspace, e.g., a typical string represented by a set of 160 bits information. This space is partitioned by a hash function such as the Secure Hash Algorithm (SHA1) [Wang et al., 2005]. Each node has a unique identifying key and is responsible for an area of the keyspace, defining the organization of the nodes in a DHT overlay network. If some information is added or queried in a DHT, this information is hashed producing a key. This key and the information from which it is derived are forwarded at each step to the neighboring node whose identifier is closer to this key. The final destination is the single node responsible for the key as specified by the keyspace partitioning.

DHTs, depending on their protocols and implementation [Lua et al., 2005], provide a near $O(\log n)$ routing performance as well as node joins and leaves for $n$ number of nodes in the overlay network. As such, DHTs provide a scalable and fault-tolerant decentralized solution for certain applications: lookup services, multimedia multicasting, and storage management systems. In contrast to lookup services of exact key matching, keyword search based on DHTs is neither easily supported nor efficient. Load-balancing and maintenance of DHTs under frequent changes is challenging. These drawbacks restrict the applicability scope of DHTs.

Hierarchical overlay networks also have a structured organization. Some representative examples include (i) super-peer overlay networks [Yang and Garcia-Molina, 2003, Garbacki et al., 2010], (ii) tree overlay networks [Tan et al., 2005a, Fei and Yang, 2007] and (iii) skip lists [Harvey et al., 2003].

Super-peer systems assign additional roles and responsibilities to a number of nodes in an overlay network, the super-peers. Their election is based on the heterogeneity of the computational, storage or network resources and mandates how the super-peer hierarchy is structured. Other factors for their election can be adopted such as the semantic content similarity in a file-sharing application [Garbacki et al., 2010]. Note that the election of super-peers is usually a domain-specific problem that is challenging to address with a generic approach or methodology [Sacha, 2009]. Moreover, super-peer overlay networks usually have power-law properties that approximate the power-law topology of the Internet resulting in improved routing performance and lower communication overhead. Super-peer systems can tolerate infrequent random failures, however, targeted attacks or super-peer failures have a significant impact on the topology that is clustered. Super-peer overlay networks may simply add another layer of complexity and overhead and, therefore, further research is required towards the possibility of super-peer overlay networks of a broader application scope.

Tree overlay networks are a subject of this thesis. They are discussed in depth in Chapter 4. Trees can be used for their efficiency in distributed operations such as 
information dissemination and aggregation. Performing these operations with trees is applicable for multimedia multicasting [Tan et al., 2005a] and distributed databases [Jagadish et al., 2006b]. However, note that trees require application-specific topology optimization and continuous topology adaptations in large-scale dynamic decentralized environments.

Finally, skip lists [Harvey et al., 2003] are randomized balanced tree data structures built by multiple levels of increasingly sparse linked lists. Based on this concept, overlay networks can be organized in various complex indexing structures such as skip graphs [Aspnes and Shah, 2007] or skip tree graphs [Beltran et al., 2007] providing flexibility and efficiency in applications of distributed databases and storage management systems. Nevertheless, an overlay network based on skip lists requires a complex building and maintenance in a distributed context.

Another structuring approach that can be applied in overlay networks is clustering [Koloniari and Pitoura, 2012, Singh and Haahr, 2007, Cantin et al., 2008, Ogston et al., 2004, Jelasity et al., 2009]. Clustered overlay networks shrink the search space of neighboring nodes and perform efficient information exchange within groups of nodes that share common properties [Ogston et al., 2004]. There are various abstract and generic clustering algorithms for overlay networks. For example, Singh and Haahr [2007] illustrate the applicability of the Schelling's social model for decentralized clustering. This clustering approach is abstract in the sense that it is not based on any domainspecific criteria. The main challenge in clustering methodologies is the adaptation of clusters under dynamically changing clustering criteria. Most related methodologies assume recomputations of the clusters that is usually cost-ineffective. However, Koloniari and Pitoura [2012] study dynamic clustering for content-sharing applications and under varying clustering criteria using a game-theoretic approach. Liu et al. [2010] illustrate this potential in a particle swarm optimization approach. Note that clustering can be used as a supportive and optimization structure in other topologies of overlay networks and distributed applications.

\subsubsection{Unstructured overlay networks}

The Gnutella overlay network, as described by the Gnutella 0.4 protocol specification ${ }^{2}$, is one of the first decentralized and unstructured overlay networks [Stutzbach et al., 2008]. It has been a reference system for other decentralized systems and its variations. Gnutella is mainly applied to file-sharing peer-to-peer systems. The overlay network of Gnutella is built as a random graph with graph properties that support flooding queries based on which users discover content in a decentralized fashion. However, due to the heterogeneity of the participating hosts and user profiles, the topology of Gnutella evolves to a power-law graph [Stutzbach et al., 2008]. Furthermore, the original flooding mechanisms of Gnutella are unscalable and result in a significant communication overhead. This drawback has limited its applicability to file-sharing applications.

In contrast to Gnutella 0.4 that is based on a statically organized unstructured overlay network, gossip-based overlay networks are highly dynamic systems of information dissemination [Jelasity et al., 2007, Kermarrec and van Steen, 2007]. Their topology changes continuously but remains random and highly connected even under catastrophic failures in an overlay network. Gossiping protocols, also referred to as epidemic

\footnotetext{
${ }^{2}$ Available at: http://rfc-gnutella.sourceforge.net/developer/stable/index.html (last accessed: March 2012)
} 
protocols, usually define periodical interactions in a 'push', 'pull' or 'push-pull' pairwise fashion to exchange information [Jelasity et al., 2007]. Nodes of most gossiping protocols execute three basic tasks [Kermarrec and van Steen, 2007]: (i) selection of nodes with which gossiping is performed, (ii) selection of the information exchanged during gossiping and (iii) processing of the received information based on application-related criteria. Gossiping protocols provide robust information dissemination, collection and discovery. They are also a generic solution and basic component in various large-scale distributed applications such as multimedia multicasting [Leitao et al., 2007] and wireless sensor networks [Modiano et al., 2006]. For example, the peer sampling service of Jelasity et al. [2007] is a generic mechanism that unifies a wide range of gossiping protocols in one framework and provides, under various network conditions, random samples of information distributed in an network. Although such a mechanism can be reused by several applications, random samples usually require some form of structuring, e.g., classification. Therefore, additional mechanisms need to be employed for this purpose.

Beyond Gnutella and gossiping, unstructured overlay networks can be built by various mechanisms that employ some random node selection. For instance, Vishnumurthy and Francis [2006] study various intelligent random walks that build unstructured overlay networks by considering heterogeneous computational or storage capacities in a network. Similar intelligent random walks are introduced by Scholtes and Tessone [2012] in an organic design of massive unstructured overlay networks studied from a complex systems perspective. The authors introduce a statistical mechanics approach based on thermodynamic laws to build and maintain unstructured overlay networks of certain graph properties.

\subsubsection{Comparison}

The organizational approaches of structured and unstructured overlay networks and their applications exhibit different properties and trade-offs. Figure 2.2 summarizes some of the most important ones that have also been identified in literature [Lua et al., 2005, Castro et al., 2005, Yang et al., 2006, Scholtes and Tessone, 2012].

- Complex us simple design: Structured overlay networks have more complex organizational constraints and requirements than unstructured ones. Satisfying structural constraints and meeting organizational requirements in dynamic distributed environments is challenging. For example, concurrency or load balancing issues need to be addressed when nodes join, or leave as discussed by Scholtes and Tessone [2012]. In contrast, unstructured overlay networks built and maintained by, for example, gossiping protocols are asynchronous and introduce simpler interactions.

- Specific vs abstract usage: Structured overlay networks are usually designed or optimized to perform certain operations. For example, DHTs perform information lookup and tree overlay networks perform information multicasting. This fact restricts the application scope of structured topologies, however, they are usually more efficient than unstructured ones that are abstract and exhibit random properties. Abstraction provides flexibility, however, the application development requires a higher effort for its design and optimization. 


\section{Structured
Overlay Networks}

Complex Design

Specific Usage

$$
\text { Fragile }
$$

\section{Lower Scalability}

\author{
Lower Cost
}

\section{Deterministic}

Unstructured

Overlay Networks

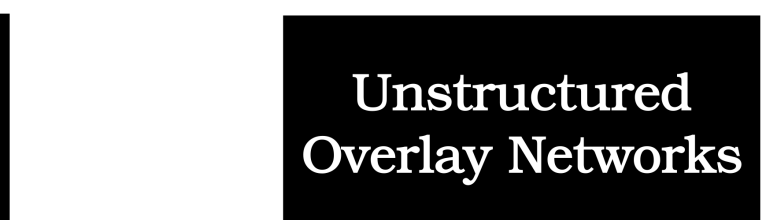

Simple Design

\section{Abstract Usage}

\section{Robust}

\section{Higher Scalability}

Higher Cost

Stochastic

Figure 2.2: A simplified comparison of structured and unstructured overlay networks.

- Fragile vs robust: Structured overlay network require explicit repair mechanisms to deal with failures of nodes. Multiple failures may disconnect the topology and result in performance degradation of applications. Repair and maintenance mechanisms make system design more complex and increase computational, storage and communication cost of a network. In contrast, robustness is usually an inherent design property of unstructured overlay networks. For example, in topologies built by gossiping protocols, node removals affect a small portion of the overlay network and the continuous topology update guarantees that the overlay network is not clustered.

- Lower vs higher scalability: In contrast to structured overlay networks with bounded scalability, unstructured overlay networks are known to be highly scalable [Scholtes and Tessone, 2012] and are able to support massive distributed systems of millions of nodes.

- Lower vs higher cost: Computational, storage and communication cost is a design consideration of structured overlay networks. Certain topologies minimize costs [Qiao and Bustamante, 2006], e.g. minimum spanning trees. In contrast, unstructured overlay networks usually exhibit higher costs due to their redundancy and randomness. For example, Gnutella uses flooding that causes the receipt of duplicate queries imposing a significant processing and communica- 
tion overhead. Similarly, gossiping protocols require continuous and periodical information exchange that can be expensive for some applications. However, note that the costs of structured overlay networks may be greater than for unstructured overlay networks under certain conditions. For example, in a decentralized, heterogeneous and unreliable environment of frequent system changes, maintenance, load balancing and repair mechanisms required for structured overlay networks may have a higher cost than the regular overhead experienced in unstructured overlay networks. Scholtes and Tessone [2012] underline this issue.

- Deterministic vs stochastic: Structured systems are designed for a purpose to have a more predictable and manageable deterministic behavior. In contrast, unstructured overlay networks are mainly stochastic as they are governed by random and emergent phenomena that may not be apparent in system design. Management and control of large-scale complex decentralized systems that experience emergent phenomena is one of the most critical future challenges that need to be addressed.

These trade-offs between structured and unstructured overlay networks show that none of these two organization types alone can fully address the challenges of future large-scale complex decentralized systems and their applications. This is one reason why overlay networks mainly remain application-dependent and domain-specific. Castro et al. [2005] and Yang et al. [2006] have shown that combining design aspects of both structured and unstructured overlay networks is an approach that potentially (i) inherits most of their beneficial properties and (ii) minimizes their drawbacks. The next section discusses the practice of using multiple overlay networks.

\subsection{Multiple Overlay Networks}

Two or more overlay networks can be coexistent or nested over a physical network as discussed at the beginning of this chapter. However, overlay networks can also be integrated. This means that one overlay network, e.g., its links, is embedded in another one resulting in a compositional overlay network with new (topological) properties and features. For example, Leitao et al. [2007] introduce a gossip-based overlay network that sends 'push' gossiping messages over tree branches to broadcast content. The rest of the gossiping links are used for tree repair. Although this method provides high performance and low cost for the specific type of tree built, it is not straightforward to generalize this approach for other types of tree optimizations and applications and even for other types of overlay networks. Often, integration results in less modular applications of overlay networks.

Multiple overlay networks may be cooperative or competitive. Cooperative overlay networks inter-support a certain distributed application by exchanging resources or information required to perform their operations. For example, Exarchakos et al. [2007] propose a capacity sharing load-balancing mechanism among multiple overloaded and underloaded structured overlay networks. Cooperative overlay networks are often modular components of a distributed application. In contrast, competitive overlay networks aim to maximize the utilization of their shared physical network and application resources [Corez and Robledo, 2011]. Zhenhai et al. [2003] introduce competitive overlay networks that allocate network resources based on Service Level Agreements (SLAs). 
There are several open issues regarding competitive overlay networks. For example, Keralapura et al. [2008] have identified race conditions and cascading effects originated by independent closed controlled loops in overlapping routing paths and periodic probing processes.

Hybrid overlay networks are usually combined structured and unstructured overlay networks designed to improve the performance of an application compared to only structured or unstructured organizational approaches. There are various proposed hybrid overlay networks in literature. For example, the second generation of Gnutella networks, as described by the Gnutella 0.6 protocol specification ${ }^{3}$, are super-peer overlay networks (structured) in which super-peers are randomly connected (unstructured). A similar approach is followed by Schmid and Wattenhofer [2007] who introduce clusters over unstructured overlay networks to improve the performance of flooding mechanisms. Moreover, Holzer et al. [2009] position multiple 'overlay graphs' as the means to improve the shortest path search for different applications. Overlay networks are decomposed to ones of fewer nodes as a way to minimize the shortest path length. Finally, the approaches of Zhao et al. [2002], Mizutani et al. [2010] and Zhao et al. [2006] combine structured only or unstructured only overlay networks as a means for redundancy and higher application performance. The actual drawbacks of the structured and unstructured overlay networks illustrated in Section 2.1.3 are not addressed.

These research efforts show that the introduction of multiple overlay networks is a design choice that enables performance enhancements and trade-offs between structured and unstructured overlay networks. In other words, multiple overlay networks are usually introduced for application-oriented optimization. In contrast to this practice, this thesis motivates the adoption of multiple overlay networks towards providing more generic application capabilities for large-scale decentralized systems. Abstraction and self-organization are crucial for this requirement as discussed by Prehofer and Bettstetter [2005], and, therefore, multi-level self-organization and control via nested cooperative overlay networks is the approach studied throughout this thesis.

\subsection{Main Concepts and Positioning}

The concept of overlay networks appears in a wide range of research areas, such as middleware, peer-to-peer, multi-agent and telecommunication systems. Each of these areas defines its own concepts and terminology to describe and model overlay networks resulting in overloaded terms and conceptual inconsistencies. For example, overlay networks are built by virtual nodes that appear to be related to 'peers', 'agents', 'hosting machines' or 'software clients' in literature. This section positions these terms in the context of decentralized computing systems of overlay networks that support different applications. Note that this positioning serves the content illustration of this thesis.

Figure 2.3 illustrates the following four entities:

1. Host: This entity is a physical machine with a network interface connected to a physical network in which one or more services of overlay networks are located.

2. Peer: This entity is a software environment that hosts agents and enables their communication.

\footnotetext{
${ }^{3}$ Available at: http://rfc-gnutella.sourceforge.net/src/rfc-0_6-draft.html (last accessed: March 2012)
} 
3. Agent: This entity is a software system that carries out, with some degree of independence or autonomy, a set of operations defined by a service of overlay networks.

4. Node: This entity is a logical abstraction and representation of an agent in an overlay network.

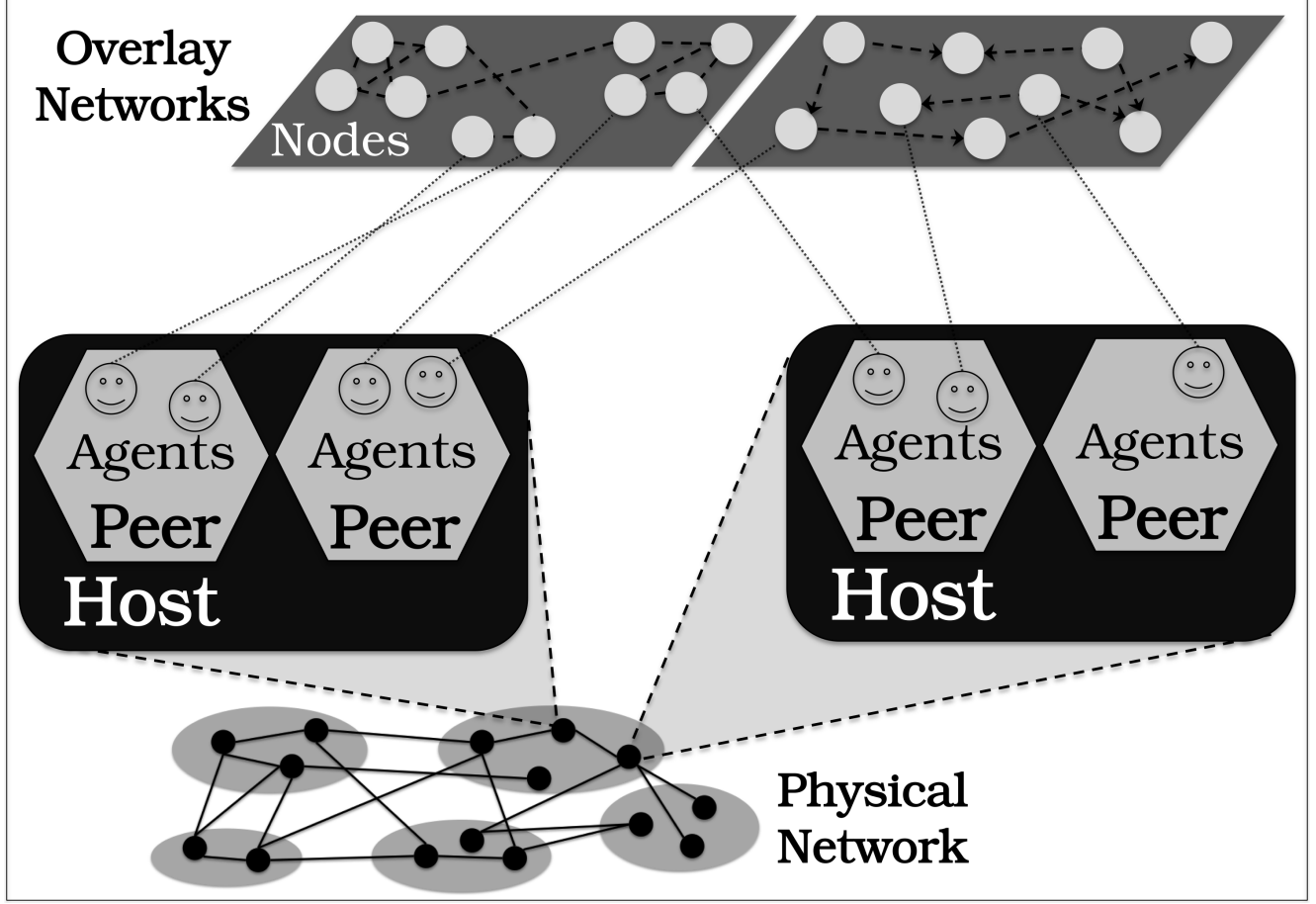

Figure 2.3: The four entities defined within the context of decentralized systems of overlay networks: (i) host, (ii) peer, (iii) agent and (iv) node.

An overlay network is a graph representation of information managed by agents. Peers and agents can be part of a middleware system or integral parts of distributed applications. As shown in Figure 2.3, a host may contain more than one peer that each may also contain multiple agents. Finally, note that an overlay network is defined by agent memberships: Every agent in a network stores unique network identifiers and other information of other agents in a limited (partial) set. The memberships known to an agent are its partial view of the system.

This thesis focuses on generic overlay network solutions that are made accessible to a wide range of distributed applications. These generic solutions are referred to in this thesis as overlay services ${ }^{4}$. An overlay service is defined as a decentralized

\footnotetext{
${ }^{4}$ In contrast to overlay services as defined in this chapter, service overlay networks (SONs), introduced by Zhenhai et al. [2003], refer to a number of dedicated hosts in ISPs that explicitly allocate resources for peer-to-peer or other decentralized systems. Bandwidth resources of certain quality are provisioned based
} 
software mechanism that provides a number of generic application capabilities of a broad application scope enabled by one or more overlay networks. Note that overlay services are often realized as distributed middleware systems.

An overlay service is characterized by its quality. The quality of an overlay service is defined as a measurable metric that quantifies the degree to which this overlay service can meet certain application objectives [Mahambre et al., 2007], for instance, the average response time [Nahrstedt et al., 2003] of queries to a directory service that relies on an overlay network.

\subsection{Prototyping Overlay Services}

This section illustrates two prototyping software environments for distributed systems and applications, the Protopeer [Galuba et al., 2009] and AgentScape [Overeinder and Brazier, 2006] platforms. These are the platforms used in this thesis for the experimental evaluation of the overlay services introduced in Chapter 4 and 5.

\subsubsection{Protopeer}

Protopeer $^{5}$ is a prototyping toolkit for event-driven simulation and live network deployment of distributed systems and applications. Its interface allows the development of an application once and its deployment in different systems without changes in the application code. Protopeer provides generic application programming interfaces (APIs) for message passing and queuing, timer operations and management of overlay networks. Figure 2.4 illustrates the architecture of Protopeer.

The Protopeer API defines a 'peer' software entity that provides the runtime context for 'peerlets'. Peerlets encapsulate the functionality of applications and correspond to the agents shown in Figure 2.3. Moreover, Protopeer supports overlay routing, bootstrapping and neighborhood management capabilities in peers ${ }^{6}$. Protopeer provides tools for logging and aggregation of measurements during runtime. Peer, time-related, or user-defined measurements can be logged locally or sent to a server for further processing or storage. This capability supports testing and evaluation of Protopeer applications.

The notion of time and networking in Protopeer is abstracted by two generic APIs respectively. This allows the usage of different network implementations beyond the Apache Mina ${ }^{7}$ that is currently supported as a backend. This flexibility also allows a distributed application implemented by Protopeer peerlets to move from simulation to a live deployment in an infrastructure such as PlanetLab ${ }^{8}$ without changing application

on SLAs. SONs provide a generic model for the allocation of Internet resources to decentralized systems and applications rather than a methodology of how to provide generic application capabilities enabled by overlay networks. Note that this distinction is identified by Haage et al. [2009] that refers to overlay services as overlay-based services. Overlay services can coexist on top of SONs. In this case, SONs provide a business model for the Internet resource allocation required for overlay services and their applications [Fan and Ammar, 2006].

${ }^{5}$ Available at: http://sourceforge. net/projects/protopeer/ (last accessed: March 2012)

${ }^{6}$ Experience with the use of Protopeer in the experimental work of this thesis shows that this support is rather limited for the overlay services introduced in this thesis and therefore overlay networks are managed within the implemented peerlets.

${ }^{7}$ Available at: http: / / mina. apache.org/ (last accessed: March 2012)

${ }^{8}$ Available at: http: / /www.planet-lab.org/ (last accessed: March 2012) 


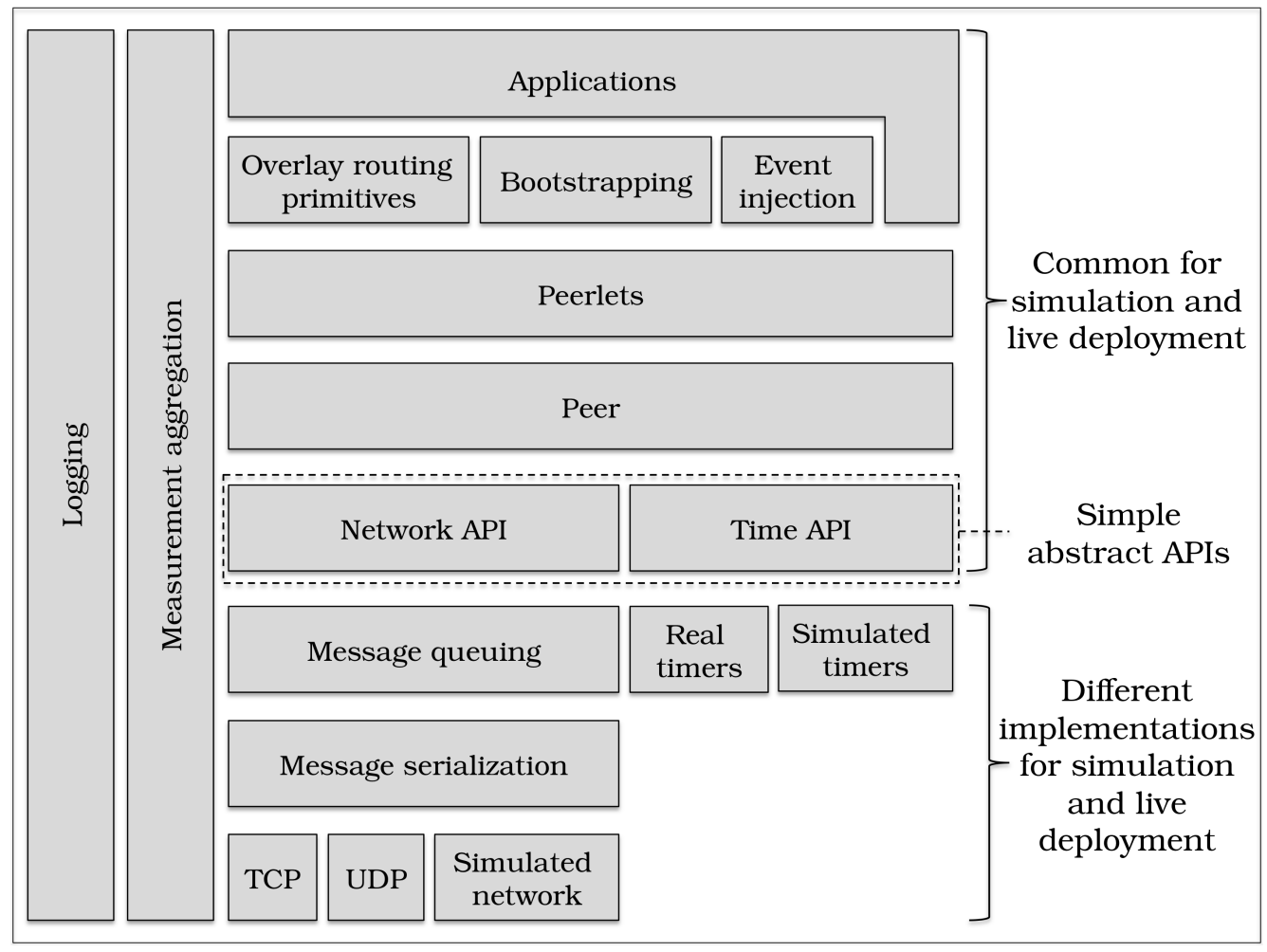

Figure 2.4: The architecture of Protopeer (adjusted from Galuba et al. [2009]).

code. The experience gained through the implementation work of this thesis shows that this assumption holds if and only if the application code is written without assumptions related to specific implementations of the network and time APIs, i.e., asynchronous communication. Therefore, the actual flexibility of Protopeer is its support to a more incremental development of distributed applications. Simulation allows a more controlled and deterministic environment without requiring a significant effort on issues such as concurrency that can be validated and addressed during the phase of live deployment.

\subsubsection{AgentScape and its simulation framework}

AgentScape $^{9}$ is a middleware platform for large-scale multi-agent systems that can interoperate with other agent platforms. The AgentScape middleware can run on different operating systems, e.g., Solaris, Linux, or Windows, and applications can be written in different code bases, such as Java, C, C++, Python and other. The AgentScape model defines two entities: (i) 'agents' and (ii) 'services'. Agents are active entities that interact with each other by message-passing. Every agent is accessible at a certain 'location' from which it can migrate to a different one based on a weak mobility ${ }^{10}$ scheme. Loca-

\footnotetext{
${ }^{9}$ Available at: http: / / www . agentscape. org (last accessed: March 2012)

${ }^{10}$ Weak mobility is the transfer of the agent code and data state from one location to another one. In contrast, strong mobility additionally transfers the execution state of the agent such as its program stack and counters.
} 
tions can be used as a default clustered overlay network on which applications manage based on their own defined criteria. Services provide information or activities on behalf of agents or the AgentScape middleware. Figure 2.5 illustrates the main concepts of the AgentScape middleware platform.

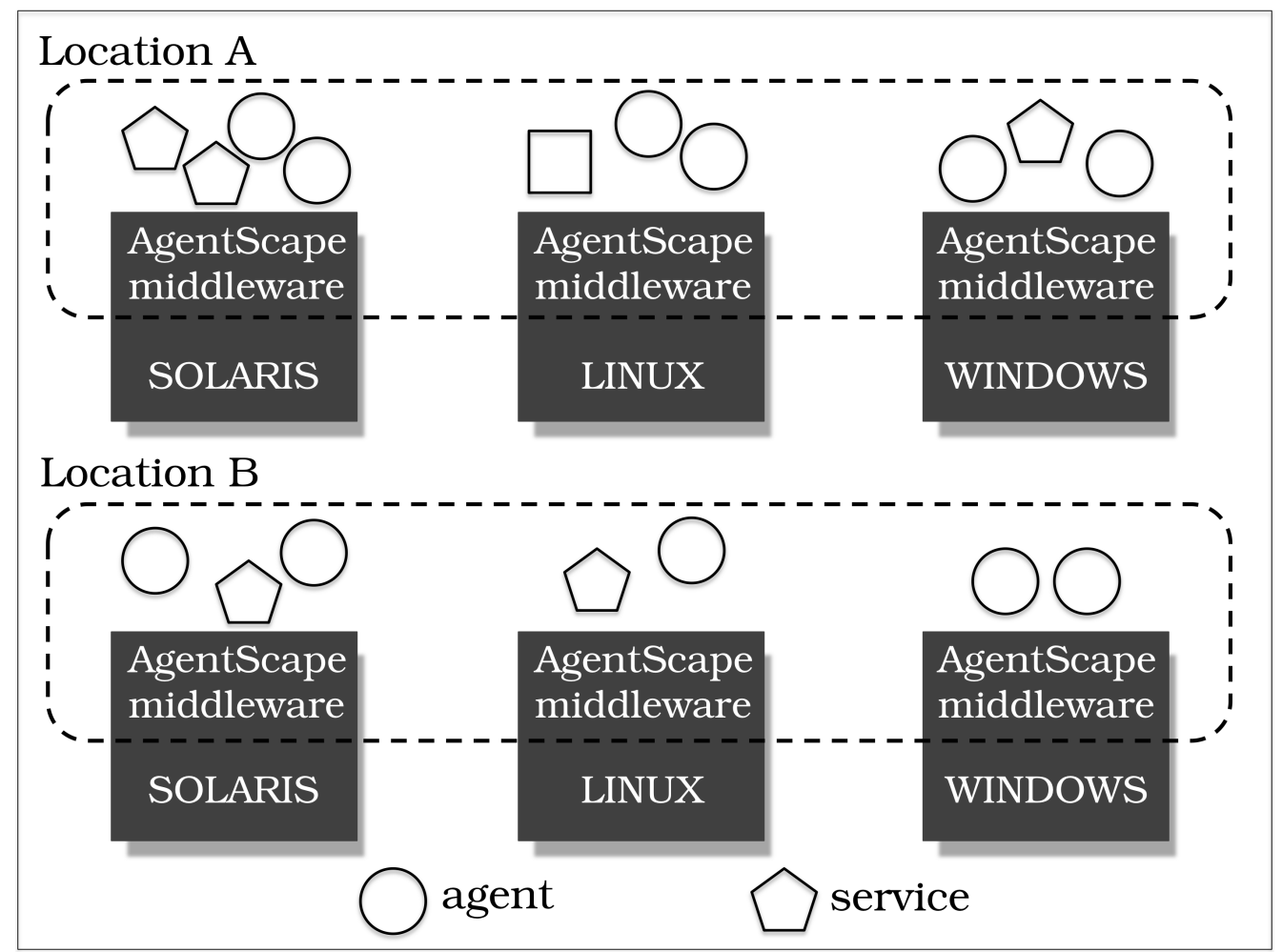

Figure 2.5: The main concepts and elements of the AgentScape middleware platform (adjusted from [Overeinder and Brazier, 2006]).

AgentScape consists of a kernel that couples AgentScape middleware with different operating systems. The kernel manages a secure communication [Oey et al., 2010b] and acts as an agent container. The AgentScape middleware provides a number of system services such as 'location managers', 'host managers', 'agent servers' and 'service servers'. These services are managed by a number of components outlined in Figure 2.6. More specifically, the location manager manages the locations of the agents and their migration based on location-wide security policies. The host manager is a representative entity of each location and performs local resource access and management based on host-wide security policies. Finally, the agent server provides to the implemented agents secure runtime support in the AgentScape middleware, whereas the service server provides service runtime support.

The AgentScape simulation framework [Oey et al., 2010a] provides incremental and gradual development lifecycle: (i) design, (ii) simulation, (iii) emulation and (iv) deployment. This approach provides a simpler system prototyping and a more effective debugging in issues related to concurrency, latency, fault-tolerance and applications. 
- Location configuration

- Location-wide security policies

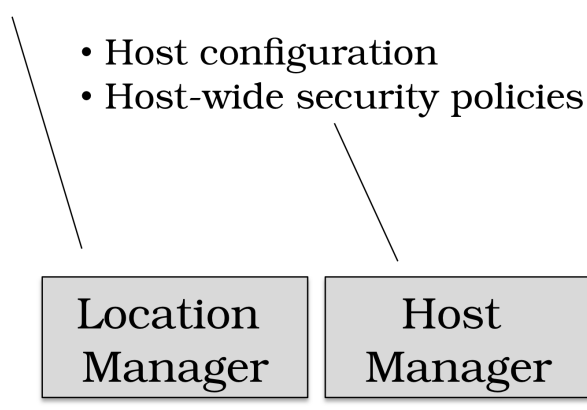

- Agent runtime support

- Sandboxing

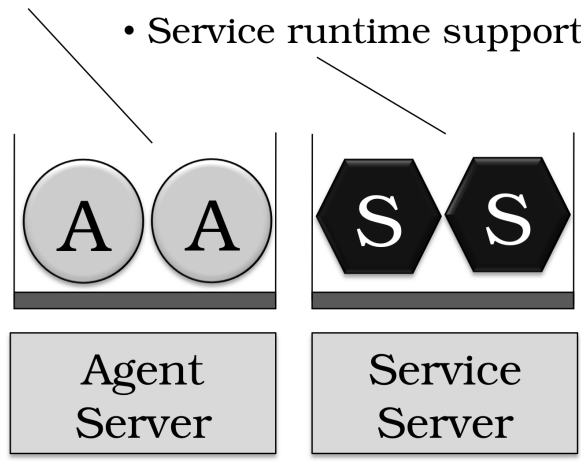

\section{AOS Kernel}

- Secure communication

- Agent container management

- Secure agent container transfer

Figure 2.6: The main architectural components of AgentScape (adjusted from [Overeinder and Brazier, 2006]).

The simulation framework of AgentScape defines a common simulation interface for multiple backends. Figure 2.7 illustrates the architecture of the simulation framework.

The simulation framework of AgentScape defines four types of backends: (i) single machine, single threaded, (ii) single machine, multi-threaded, (iii) multiple machines, multiple threads, lock-step and (iv) multiple machines, multiple threads, asynchronous. The lock-step provides some synchronization in the events generated by agents.

\subsection{Discussion and Future Work}

Research on overlay networks has generated a wide range of application-specific solutions for large-scale dynamic decentralized systems. This thesis introduces a more critical and targeted research effort towards unifying existing efforts under the umbrella of new novel and generic architectures of overlay services for Internet-based and other emerging application domains. One of the challenges for the design of such architectures is the introduction of more flexible architectural compositions between structured and unstructured overlay networks that have a more generic scope and move beyond application-specific optimizations.

This thesis does not underestimate several other open issues about overlay networks. Security issues, such as trust, privacy, fairness, Denial of Service (DoS) attacks 


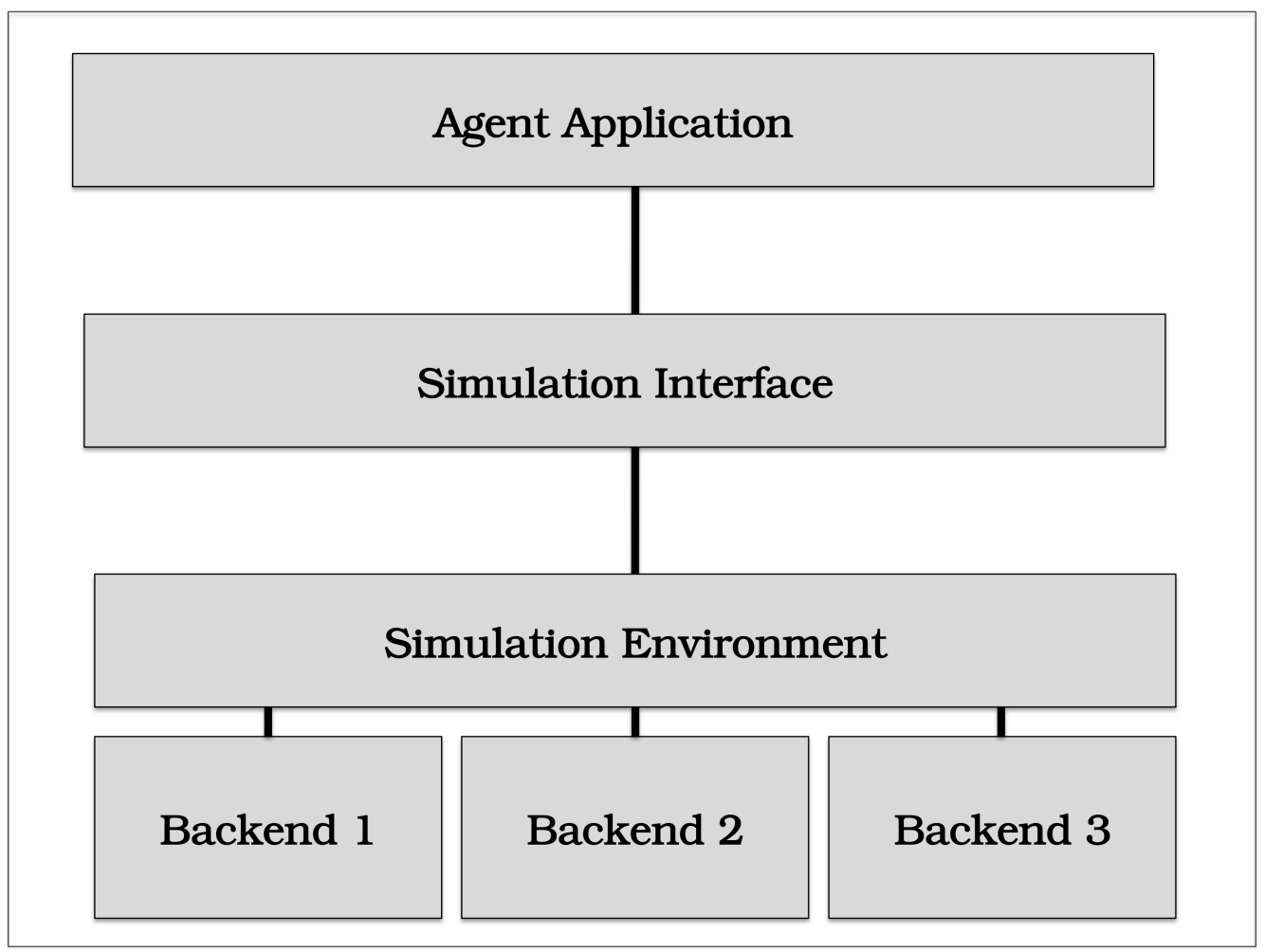

Figure 2.7: A high-level illustration of the simulation framework architecture in AgentScape (adjusted from [Oey et al., 2010a]).

and malicious nodes, require further research efforts as acknowledged by Keromytis et al. [2002], Karakaya et al. [2009], Kurian and Sarac [2010], Walters et al. [2008]. For example, Walters et al. [2008] study three types of Byzantine ${ }^{11}$ attacks in topology adaptations of unstructured overlay networks that are handled with local measurement analyses. Multiple outlier detections are performed based on which a trust model is constructed to classify the topology adaptations performed by nodes as malicious or not. These nodes are isolated to reduce their impact on the overlay network. Although security issues are not subject of this thesis, studying the further applicability of such mechanisms beyond unstructured overlay networks and certain distributed applications is crucial for realizing more generic and reliable overlay services.

Finally, the rest of this section discusses an interesting argument in literature about the role of overlay networks on the Internet and applications.

\subsubsection{The purist vs pluralist argument}

Research on overlay networks has generated an argument and discussion about their impact on future development of the Internet. Two views are supported among the re-

\footnotetext{
${ }^{11}$ In contrast to attacks such as DoS, Byzantine failures do not completely interrupt a system but result in a malfunction, corruption or inconsistency of an execution state.
} 
lated research communities: The purist and the pluralist views. Crowcroft et al. [2003], Turner and Taylor [2005], Anderson et al. [2005], Clark et al. [2006], Kurian and Sarac [2010] discuss these opposing views and are positioned, to a certain degree, concerning this argument. The purists view overlay networks as testbeds used for the implementation and experimentation of novel Internet architectures. Purists do not view overlay networks as viable or coexisting architectural elements of the future Internet in contrast to pluralists who envision overlay networks as a possible solution to deal with the heterogeneity of applications and the business challenges that ISPs experience. Pluralists support the idea that the development and adoption of technological innovations in the Internet infrastructure have become a hassle for the current business model of ISPs and, therefore, overlay networks will unavoidably remain a growing feature of the Internet.

The knowledge about overlay networks transferred from the domain of Internetbased computer networks to other application domains and socio-technical infrastructures such as the Smart Power Grid [Birman, 2001, Fan et al., 2010, Erol-Kantarci et al., 2011], wireless sensor networks [Hui and Culler, 2008] and intelligent transportation systems [Rybicki et al., 2007, Zhang et al., 2010], shows that overlay networks evolve as a design artifact for complex, large-scale, dynamic decentralized systems and their applications. Principal operations such as information dissemination, decision-making, aggregation, lookup and search have been studied as overlay-based solutions [Zhuge and Feng, 2008, Chakravarti et al., 2006, Gkantsidis et al., 2006, Jelasity et al., 2007, Van Renesse et al., 2003, Yang et al., 2006]. The aforementioned emerging application domains become more information-driven, cyber-physical, ambient and heterogeneous and therefore have similar operational requirements that can be met by overlay networks. This thesis is an example that shows this extended and generic scope of overlay networks by studying in Chapter 6 the applicability of generic overlay services in the Smart Power Grid.

Although the previous observation seems to favor the pluralists, puristic views have a lot to gain from encouraging overlay services as a design artifact for complex decentralized systems and applications. This is because overlay networks designed as integrated mechanisms in distributed applications result in more complex and less modular applications. This is a practice in which technological innovation in the Internet or other underlying network infrastructures is not a main concern or is a secondary priority. In contrast, an overlay service that is reused by different distributed applications shows the roadmap and guides the future technological adoption in the underlying physical network. Such an overlay service unifies existing application-level solutions and shows the acceptance of a current application practice towards a more broad technological adoption and support.

Both of the purist and pluralist views on overlay networks provide invaluable insights about the future development and sustainability of the Internet. This debate provides more structured observations of the current challenges and the future issues that need to be addressed. Therefore, neither of these two views should be neglected. Nonetheless, the contributions of this thesis are mainly positioned within the pluralistic view. Future work should show the actual impact of these contributions from the purist's viewpoint. 


\subsection{Conclusions}

This chapter concludes that designing generic overlay services for large-scale decentralized systems requires a new conceptual architecture about their organization and use. The current design of overlay services is highly optimization-driven and applicationoriented, especially for overlay services of hybrid overlay networks. Shifting this current practice towards more generic overlay services extends the scope of overlay networks in new emerging application domains and also promotes future architectural Internet innovations. Both requirements are supported by puristic and pluralistic views about the future impact of overlay networks. 



\section{Chapter 3

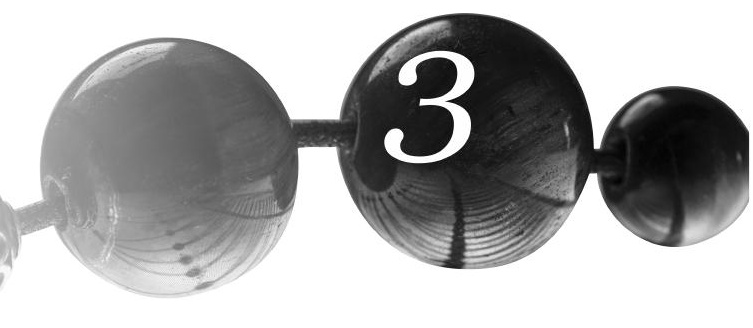

\section{Adaptive Self-organization in a Multi-level Architecture}

"The strongest is necessity, for it masters all."

Thales of Miletus ${ }^{1}$

This chapter introduces ASMA, the Adaptive Self-organization in a Multi-level Architecture. ASMA is a conceptual, multi-level, self-organization architecture that can be used to design and build overlay services as a decentralized middleware system. An overlay service of ASMA can be designed independently of an application. It is positioned as a stand-alone software system between an underlying network and different applications to provide generic application capabilities.

Multi-levelness in a hierarchical organization is the key property of ASMA and the one that provides abstraction, modularity and customization for different applications. ASMA is composed of three hierarchical self-organization levels that provide discovery, structuring and coordination of information distributed in a network. Feedback loops of continuous inter-level interactions provide a dynamic adaptation when this information changes during runtime of an overlay service. Every level provides some transparency to the level above and together they compose a service that can be used by different applications.

This chapter is organized as follows: Section 3.1 illustrates the ASMA architecture. Section 3.2 shows how this architecture is coupled to distributed applications and how its services are utilized. Section 3.3 introduces two overlay services studied in this thesis. Section 3.4 compares ASMA with related architectures, frameworks and methodologies. Section 3.5 discusses the ASMA architecture. Finally, Section 3.6 concludes this chapter.

\subsection{Architecture Overview}

ASMA is a conceptual self-organization architecture with which different generic overlay services can be designed. The implementation of an individual overlay service in the

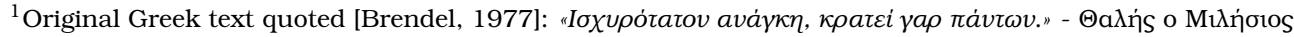


ASMA architecture is referred to as architecture realization. An architecture realization entails the realizations of tasks defined in ASMA.

ASMA addresses the challenges of abstraction and dynamic adaptation in overlay services by introducing (i) a multi-level self-organization architecture and (ii) inter-level interactions. The complexity of overlay networks is managed by multiple applicationindependent levels, each with a specific self-organization goal. Each level in ASMA supports the level above and configures the level below. These bottom-up and top-down inter-level interactions tune the self-organization operations in each level to improve the quality of an overlay service as defined in Section 2.3.

Figure 3.1 illustrates the ASMA architecture in a single peer. The design of an overlay service in ASMA is defined by sets of (i) criteria and (ii) samples within three application-independent reconfigurable self-organization levels: (i) the discovery level, (ii) the structuring level and (iii) the coordination level. For each set of criteria at each level in the architecture, a set of samples is generated. The discovery level discovers required information in the network. The structuring level structures this information. Finally the coordination level uses the structured information to build and provide the overlay service to an application.

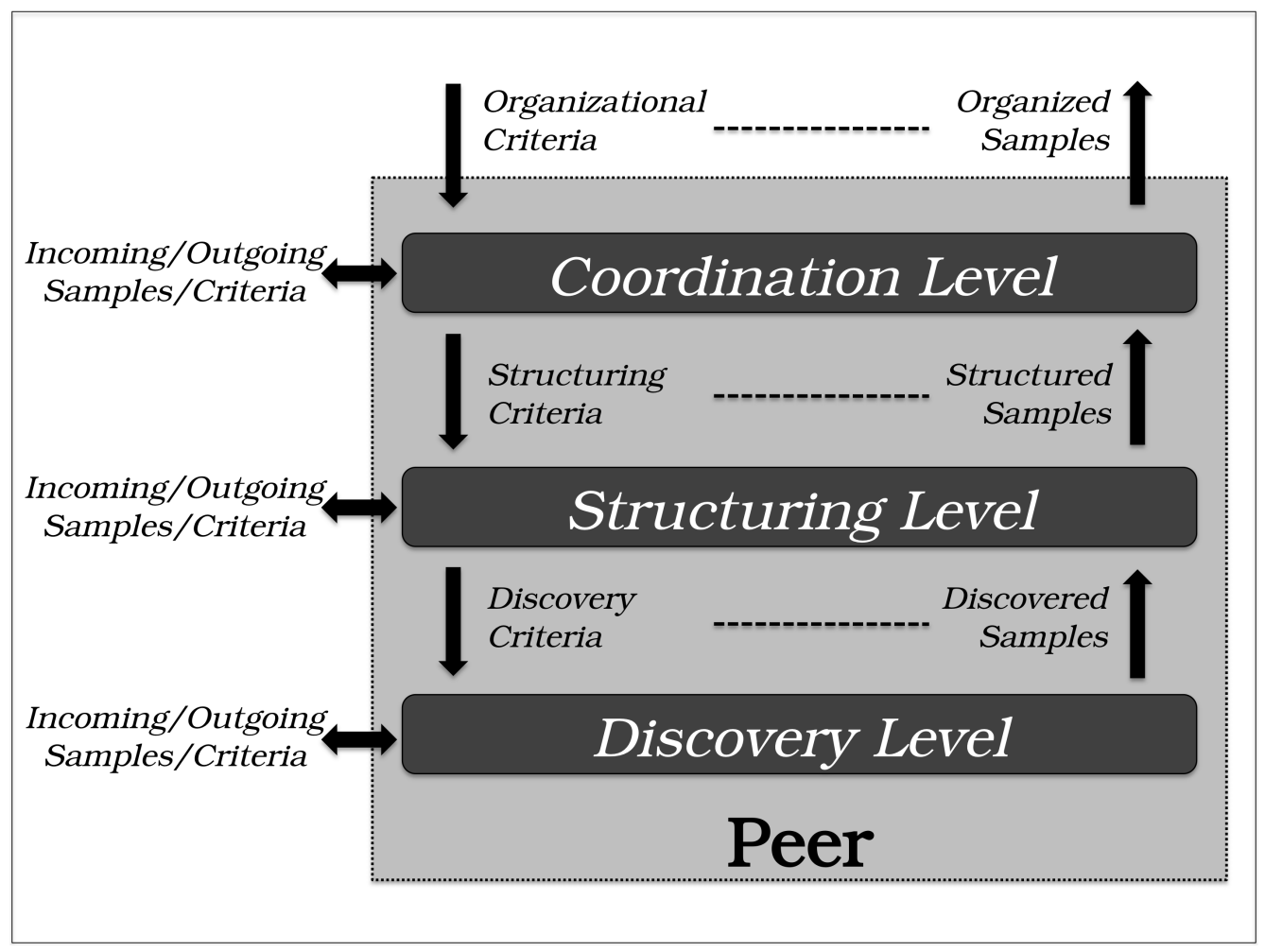

Figure 3.1: The three levels of the ASMA architecture in a peer.

A realization of the ASMA architecture is positioned within the runtime environment of a peer. Each level of this architecture is managed by one or more autonomous agents. Note that two corresponding levels in two different peers are able to communicate re- 
motely. In other words, agents of the same type are able to remotely interact. ASMA defines two types of interactions: (i) vertical and (ii) horizontal. A vertical interaction is the (local) exchange of criteria and samples between two different levels of ASMA located within the same peer. In contrast, a horizontal interaction is the (remote) exchange of criteria and samples between the same two levels of ASMA located within two different peers.

A criterion is runtime feedback information that parameterizes the operation of a level in the architecture. In vertical interactions, criteria are provided in a top-down fashion: from each level to the level below. In horizontal interactions, criteria are provided by a remote corresponding level. A criterion is generated based on some given samples and can be practically any information related to a framework realization. Criteria can be generated by a change in the value of a monitored metric, changes in the underlying network or the result of an agent negotiation. ASMA defines three types of criteria in its vertical interactions:

- Organizational criteria: These are criteria that parameterize the self-organization operation of an overlay service. They are externally provided by an application as input to the coordination level.

- Structuring criteria: These are criteria that parameterize the structuring of samples to improve the quality of an overlay service. They are provided by the coordination level to the structuring level.

- Discovery criteria: These are criteria that parameterize the dissemination and collection of samples in a network. They are provided by the structuring level to the discovery level.

A sample is continuously updated information required for the operation of a level in the ASMA architecture. In vertical interactions, samples are provided in a bottom-up fashion: from each level to the level above. In horizontal interactions, samples are provided by a corresponding level. A sample is defined within the context of an overlay service and may represent a wide range of information related to any of the entities of Figure 2.3: from information about the local host, such as its IP address or its geographic location, to information about the user, such as his/her reputation and trust in an online community. This information is usually abstracted from the application. For example, the reputation of a user in an online community can be represented as an abstract rank value of a node in the overlay network of this community. Three types of samples are defined in the vertical interactions of ASMA:

- Discovered samples: These are samples discovered in the network that are locally provided to the structuring level in which they are managed.

- Structured samples: These are discovered samples required for building an overlay service. They are provided by the structuring level to the coordination level.

- Organized samples: These are the output samples achieving a certain quality of an overlay service. They are provided by the coordination level to the applications.

The criteria and samples illustrated above are application-independent and are abstracted from the application as illustrated in Section 3.2. Samples are provided from 
the one level to the other if a condition is satisfied. Criteria are feedback parameterization triggered by the consumption of sample in a level of the architecture.

The criteria and samples exchanged in horizontal interactions are referred to as (i) incoming criteria, (ii) incoming samples, (iii) outgoing criteria and (iv) outgoing samples. The semantic of these samples and criteria is defined by an architecture realization.

\subsubsection{The architectural levels}

Each individual level of ASMA is defined according to Figure 3.2. Algorithms 3.1 and 3.2 illustrate event generations and reactions for each ASMA level. Assume an arbitrary ASMA level that (i) generates some arbitrary output criteria and samples and (ii) reacts to some arbitrary input criteria and samples. Input criteria trigger execution of the adapt task that generates the output samples. Similarly, input samples trigger execution of the consume task that generates output criteria. The provide task sends the output samples to the level above and the configure task sends the output criteria to the level below. Samples and criteria can also be sent to a remote corresponding level in a horizontal interaction. However, the provide task may call the consume task of the same level instead of the one in the level above. Similarly, the configure task may call the adapt task of the same level instead of the one in the level below. These internal calls, within a level, are possible options defined within a realization of an ASMA level. The realization of a level is defined by the implementation and scheduling of its tasks.

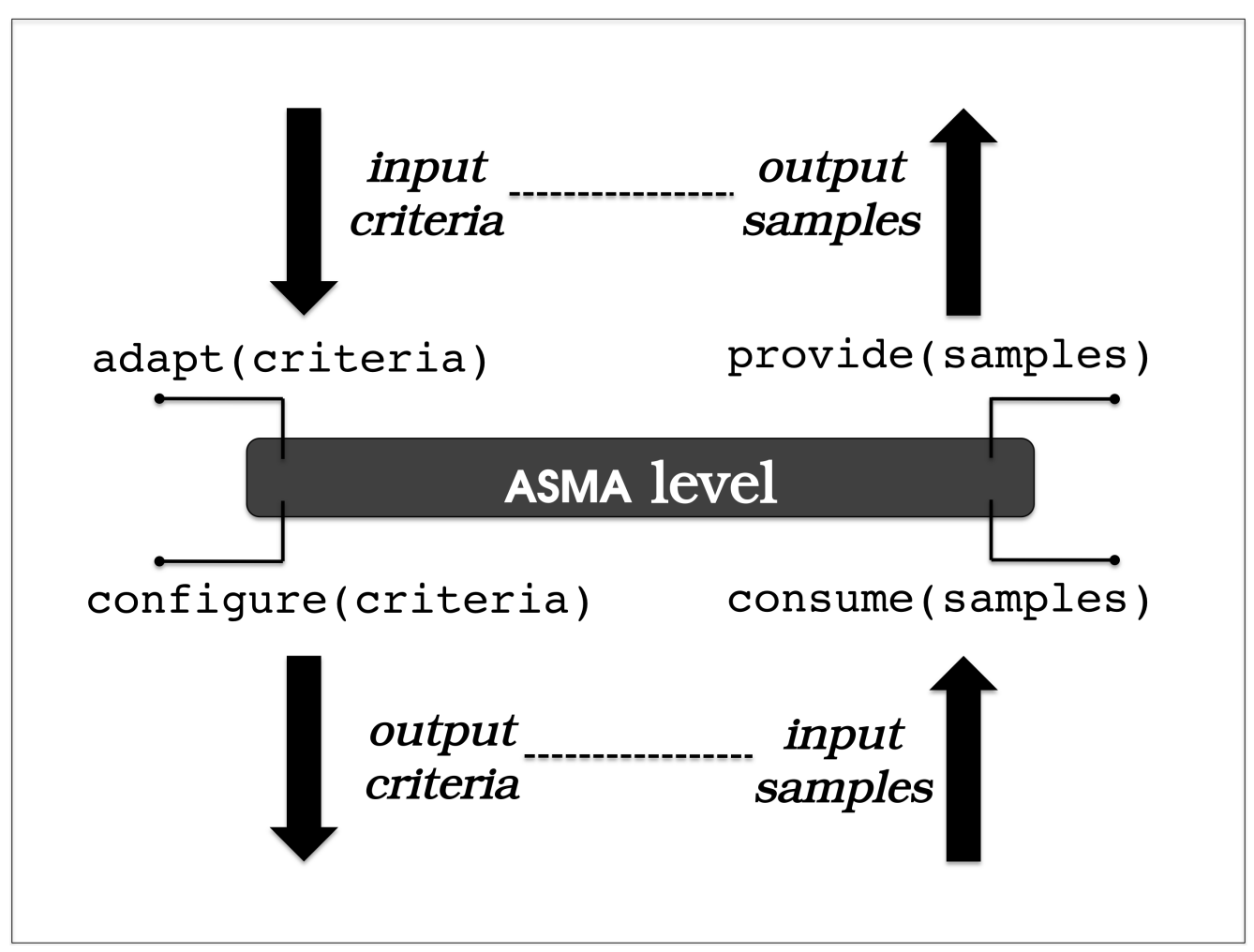

Figure 3.2: The executed tasks of an abstract ASMA level. 


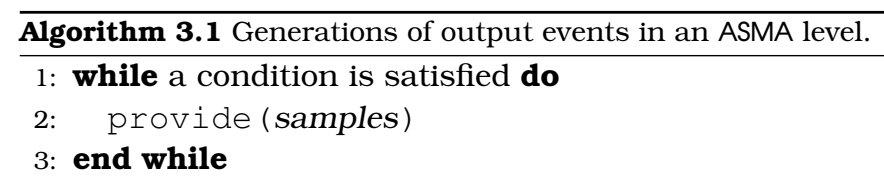

Ensure: output

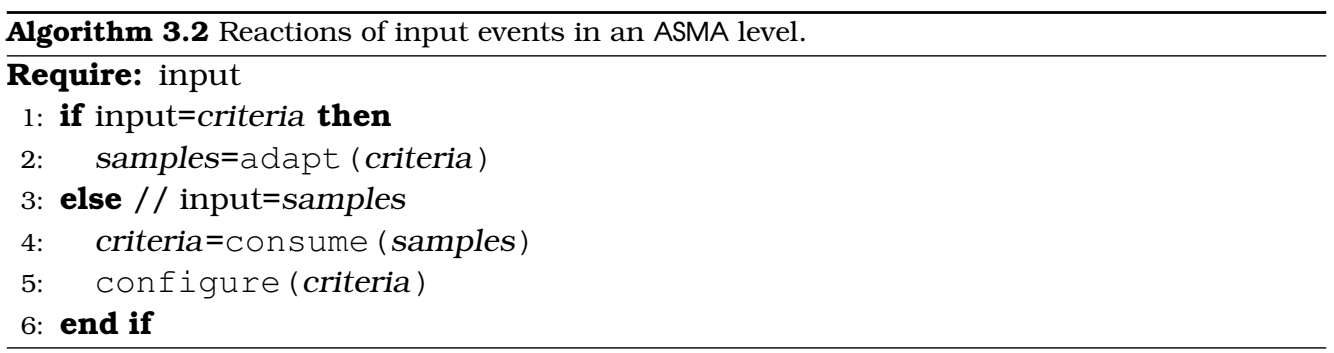

The three levels of ASMA are summarized as follows: The discovery level, positioned at the bottom of the architecture, performs discovery of remote samples required by an overlay service. Sample discovery is achieved by horizontal interactions that disseminate outgoing criteria and trigger the remote collection of incoming samples from the network. The dissemination and collection of samples is parameterized by the discovery criteria received from the structuring level. The structuring level structures the discovered samples provided by the discovery level or other incoming samples received by horizontal interactions. The structuring criteria customize the structuring and selection of the structured samples provided to the coordination level. Finally, the coordination level coordinates the main functionality of an overlay service according to a set of organizational criteria. The coordination level uses the structured samples or other incoming samples received by horizontal interactions to update the organized samples provided to an application. The quality of an overlay service achieved with certain structured samples is evaluated resulting in a new set of structuring criteria that trigger new structured samples to improve the quality of an overlay service.

Note that the organized samples are a result of a continuous inter-level adaptations and optimizations between the three levels of ASMA. The rest of this section illustrates each level of the ASMA architecture in detail.

\subsubsection{The discovery level}

The discovery level is responsible for the distribution and availability of samples to every peer of the hosts of a network providing the abstraction of sample discovery in the structuring level. The dissemination of outgoing samples in the network is performed using outgoing criteria and the configure task. Symmetrically, the collection of remote incoming samples triggers the consume task. Furthermore, the execution of the adapt task is triggered by incoming criteria and by the discovery criteria that configure the dissemination and collection of samples in favor of the structuring level. Note that remote communication between peers is possible as the samples disseminated and collected in the network contain routing information, e.g. the IP address and port number. 
Making distributed samples locally available to the peers of a network is challenging and crucial for building decentralized overlay services. Middleware systems based on centralized information lookup or distributed lookup mechanisms designed with specific applications types in mind cannot always support scalable and generic overlay services. ASMA introduces the discovery level in the foundations of the architecture to bridge the information gap of decentralization in overlay networks.

This thesis focuses on a gossip-based realization of the discovery level within the context of the overlay services studied in this thesis. Gossiping is a simple and generic probabilistic communication model according to which agents exchange samples in a 'push', 'pull', or 'push-pull' fashion [Jelasity et al., 2007]. The exchange of samples is random to certain degree but other more intelligent policies can be applied as well. Gossiping information is spread in an epidemic fashion within a network [Van Mieghem et al., 2009, Tang et al., 2011]. Furthermore, gossiping is able to prevent clustering of a network by cascading failures of its hosts.

Algorithm 3.3 and 3.4 illustrate a high-level description of a 'push-pull' gossiping protocol that realizes the horizontal interactions of the discovery level. The outgoing criteria represent a 'push' message and the incoming samples represent a 'pull' message. Both contain local discovered samples that are exchanged in a gossiping fashion. A gossip-based discovery level periodically sends outgoing criteria to a remote discovery level of a selected peer defined within these criteria (line 3 of Algorithm 3.3). The discovered samples are also provided periodically to the structuring level (line 2 of Algorithm 3.3). Furthermore, the discovery level reacts to incoming criteria by adapting its discovered samples and providing in return outgoing samples (line 2-3 of Algorithm 3.4). The incoming samples are consumed and generate the next outgoing criteria (line 5 of Algorithm 3.4).
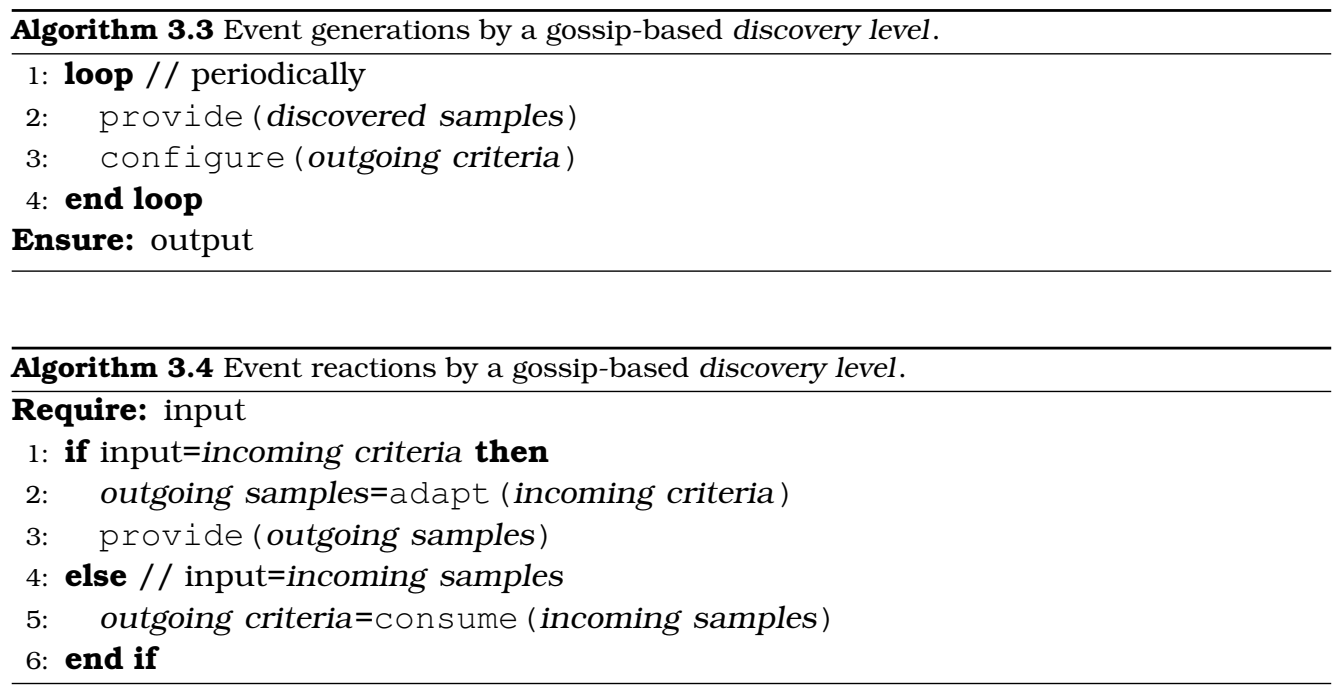

The core gossiping operations are performed in the adapt and consume tasks illustrated in Algorithm 3.5 and 3.6. The adapt task (i) handles incoming criteria that are actual 'push' gossiping messages and (ii) generates outgoing samples that are actual 'pull' gossiping messages (line 1-5 of Algorithm 3.5). The discovery criteria parameterize gossiping by, for example, selecting policies [Jelasity et al., 2007] that 
tune the dissemination of samples under various network conditions, e.g. failures in hosts. The consume task updates the discovered samples with incoming samples (line 1 of Algorithm 3.6) and selects outgoing samples to disseminate from the discovered samples (line 2 of Algorithm 3.6).
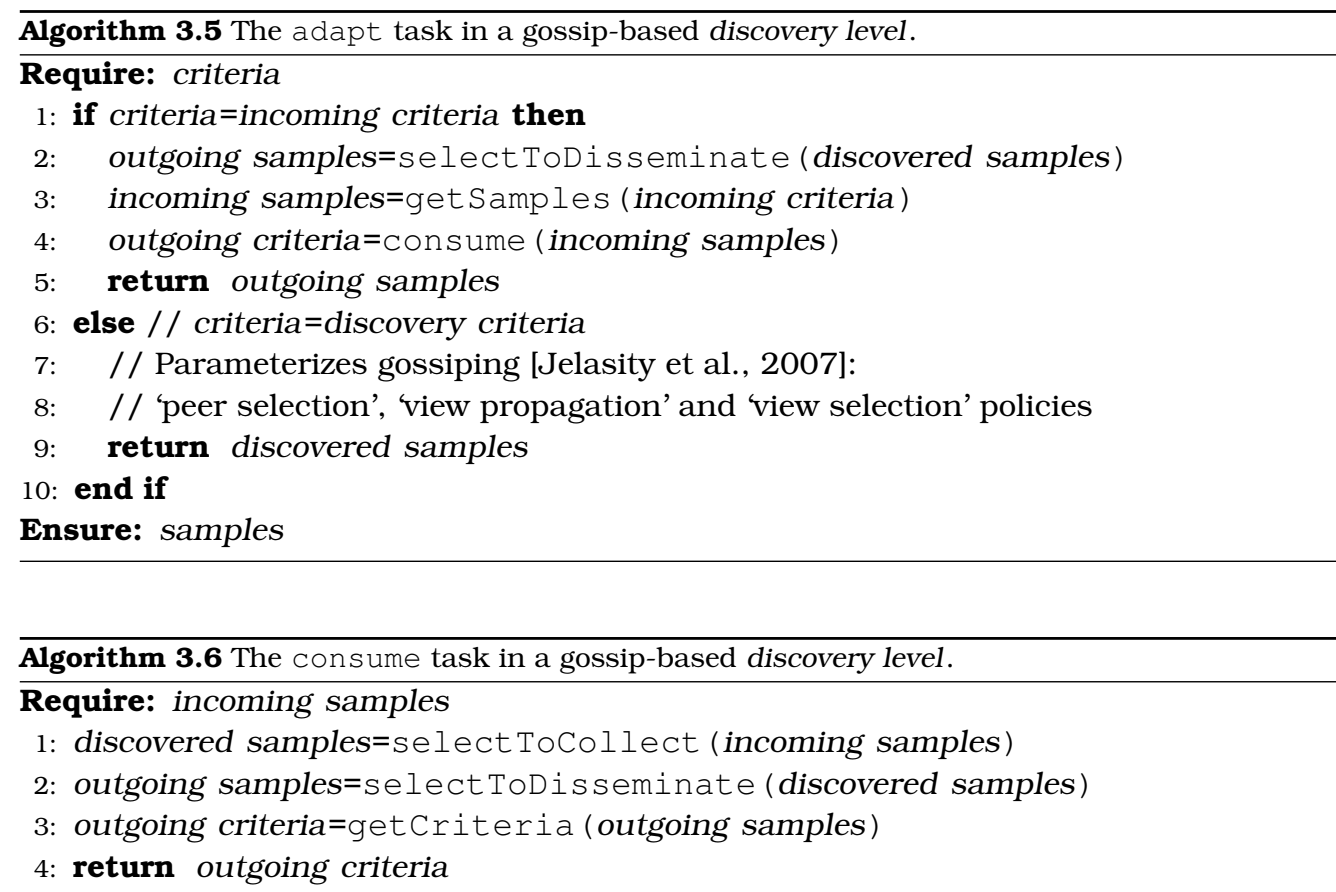

Ensure: outgoing criteria

Finally, the selectToCollect task (line 1 of Algorithm 3.6) corresponds to the respective selection task of the peer sampling service. A detailed realization of this task is illustrated by Jelasity et al. [2007]. The implementation of the selectToDisseminate task (line 2 of Algorithm 3.5 and 3.6) is also illustrated in that work. The getCriteria task performs the selection of the agent to gossip with (line 3 of Algorithm 3.6) and the get Samples task simply derives the incoming samples included in a set of incoming criteria (line 3 of Algorithm 3.5).

Other mechanisms for discovery such as flooding [Jiang et al., 2003], random walks [Gkantsidis et al., 2006] and DHT overlays [Yuh-Jzer et al., 2005] are not excluded from the architecture as they can also achieve decentralized dissemination and collection of samples. However, these mechanisms require a high customization and DHTs need maintenance that makes their utilization more complex in the context of a generic self-organization architecture for overlay services. For this reason, a gossipbased realization of the discovery level is chosen and studied in this thesis.

\subsubsection{The structuring leve1}

The structuring level is responsible for the management of discovered samples, providing in this way an abstraction to the coordination level. More specifically, the structuring level performs (i) structuring, such as sorting, clustering and classification of the 
discovered samples received from the discovery level and (ii) selection of the structured samples provided to the coordination level.

Structuring and selection are based on criteria defined by an adaptation strategy. An adaptation strategy is a set of reconfigurable parameters that define these criteria. The following three examples illustrate some adaptation strategies:

- Sorting a list of ranked samples in an ascending order and selecting the first sample from the list.

- Clustering a set of ranked samples based on their ranking distance and selecting the highest or lowest ranked sample in each cluster.

- Classifying a set of samples in a number of classes and selecting the most recently added sample from each class.

There is a wide range of adaptation strategies that can be designed regarding a certain self-organization goal supporting an overlay service. Note that adaptation strategies provide dynamic management of samples as:

- Multiple adaptation strategies can be designed and adopted dynamically during runtime.

- The parameters of an adaptation strategy that define the structuring and selection of samples can be reconfigured during runtime.

Both approaches can be enabled based on structuring criteria received from the coordination level. The structuring criteria result in new structured samples that potentially improve the quality of an overlay service. In this case, the structuring criteria are an actual feedback about the provided structured samples that can be interpreted by the structuring level as either (i) a switch to a different adaptation strategy or (ii) a reconfiguration of the parameters that define a certain adaptation strategy.

Algorithms 3.7 and 3.8 illustrate the event generations and reactions in the structuring level. Note that the tasks executed by the structuring level are specializations of an abstract ASMA level. Criteria and samples exchanged in horizontal interactions are defined in a level realization.

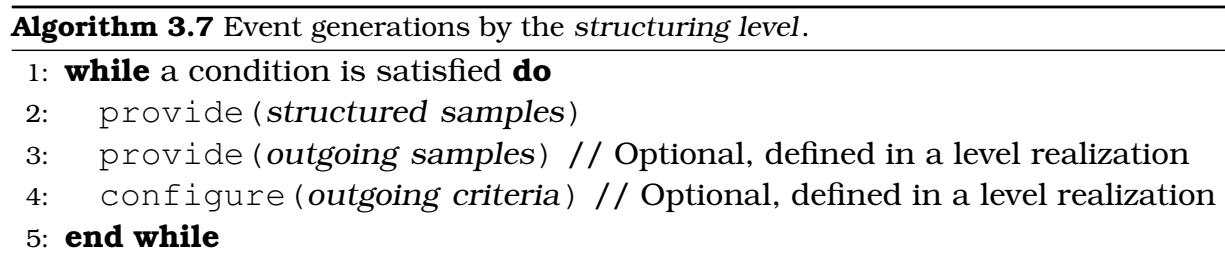

Ensure: output

Algorithms 3.9 and 3.10 illustrate the adapt and consume task in the structuring level. The adapt task is based on two subtasks, the adopt and select ToProvide. The first subtask is responsible for the selection and reconfiguration of the adaptation strategy based on which the structured samples are selected (line 2 of Algorithm 3.9). A learning or rule-based system may be used to correlate certain feedback information 


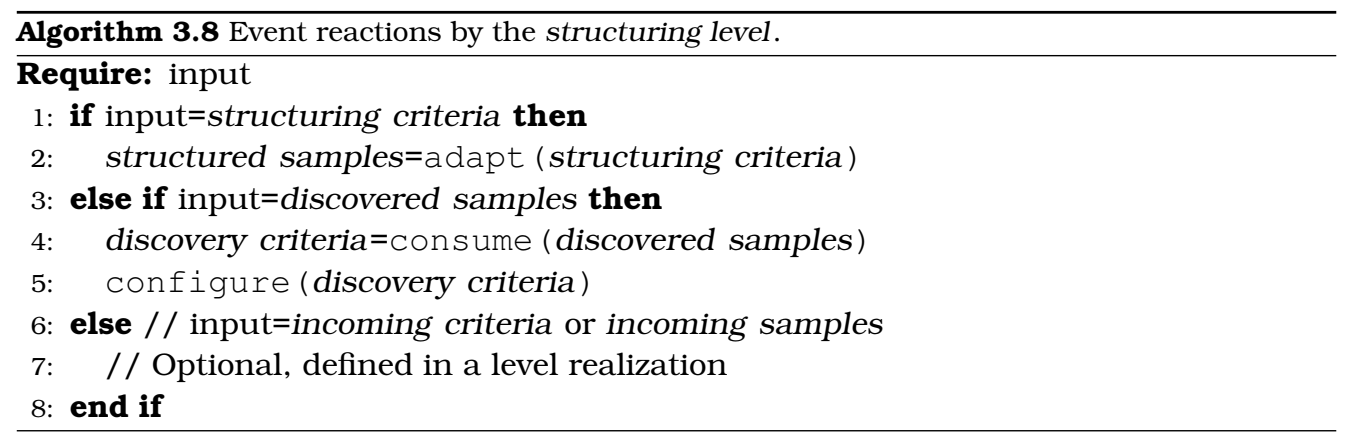

contained in the structuring criteria with a number of adaptation strategies supported by the structuring level. Furthermore, the select ToProvide subtask selects a number of structured samples provided to the coordination level (line 3 of Algorithm 3.9). The selection of structured samples is performed based on criteria defined within the selected adaptation strategy. The consume task defines the structuring of the discovered samples, such as sorting, clustering, classification, etc., based on the selected adaptation strategy (line 2 of Algorithm 3.10). The adopt, select ToProvide and structure subtasks are realized by each overlay service and therefore their definition is subject of an architecture realization
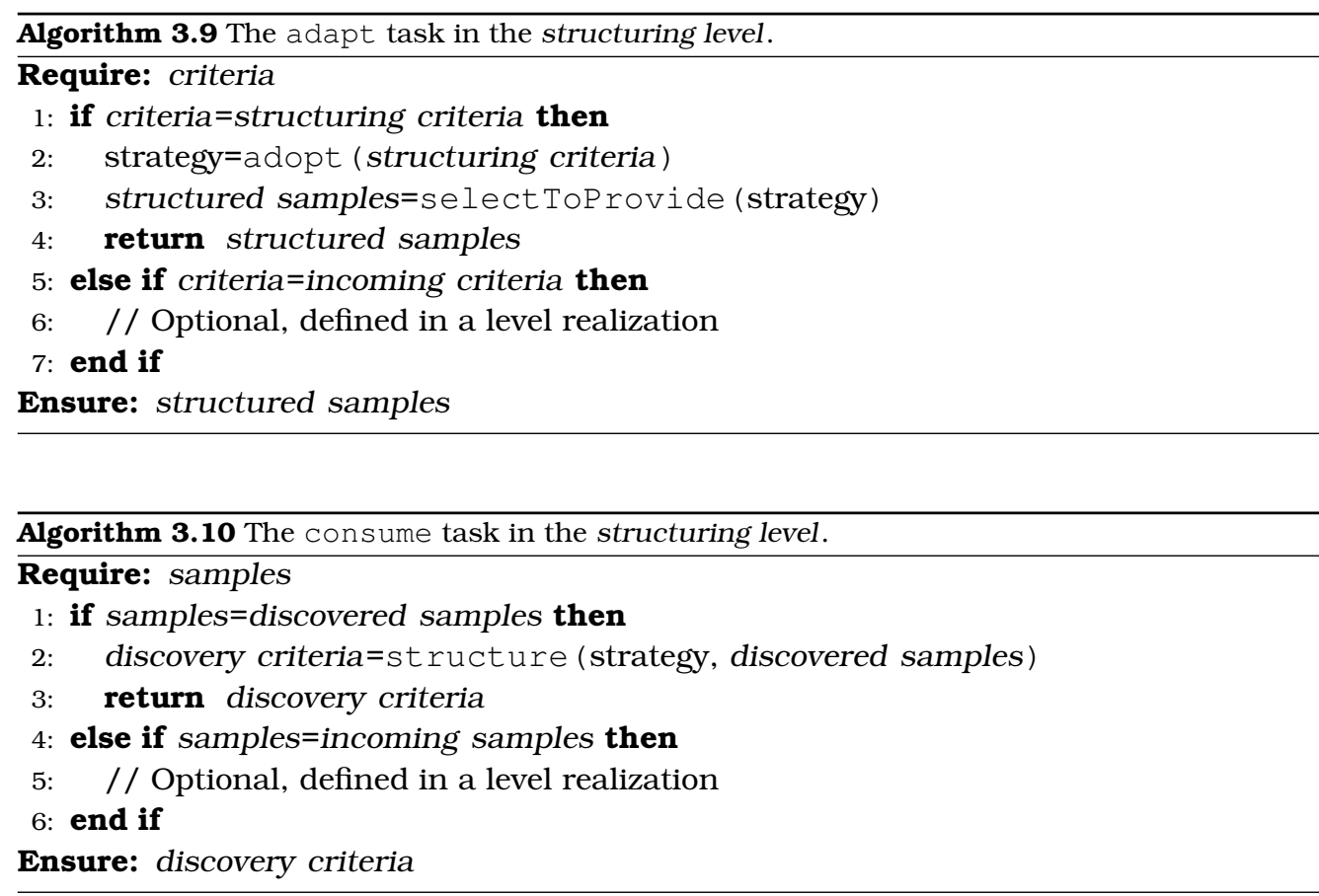

Adaptation strategies introduce a modularity level in the design phase of an overlay service. Self-organization by adopting multiple adaptation strategies or by reconfiguring a certain strategy provides a flexible compositional environment for supporting selforganization goals related to various application criteria. 


\subsubsection{The coordination level}

The coordination level is responsible for the continuous organizational update of the organized samples provided to an application. The update of the organized samples is based on the structured samples provided by the structuring level and the organizational criteria provided by an application. Updating the organized samples may require some coordination between remote agents of the coordination level. These agents are defined by the structured samples. Coordination may concern the exchange of samples required for the operation of an overlay service, a negotiation between two agents about their required samples, a query, or some other type of remote interaction and operation.

The organized samples are organized as defined by the organizational criteria. Organization can be tuned by a fitness function [Nelson et al., 2009] or another evaluation scheme that maximizes the quality of an overlay service. This process generates a set of structuring criteria containing feedback for the structuring level to trigger the next structured samples that improve the quality of an overlay service. Therefore, the exchange of samples and criteria between the structuring level and the coordination level is a continuous and iterative optimization process of the quality of an overlay service.

Algorithm 3.11 illustrates the event generations in the coordination level. The delivery of the organized samples to the application is defined by the organizational criteria (line 1 and 2 in Algorithm 3.11). The organizational criteria related with this delivery may concern a certain quality of an overlay service, or an elapsed runtime period.

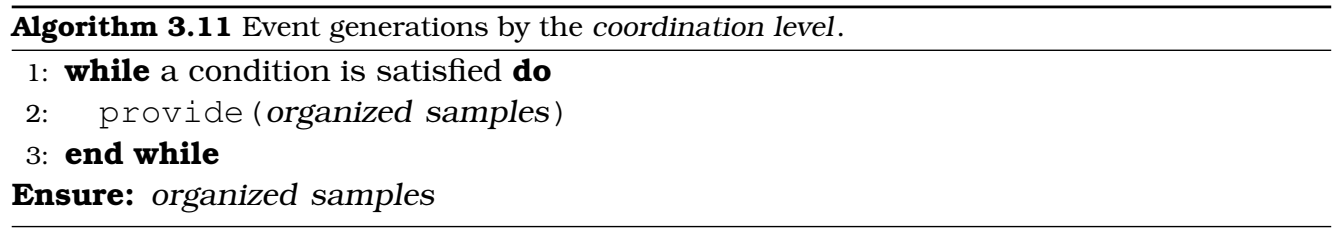

Algorithm 3.12 shows the event reactions. A simple coordination scenario of horizontal interactions is assumed in which an agent of the coordination level sends a set of outgoing criteria containing some outgoing samples and receives back incoming samples. This scenario corresponds to a coordination based on an information exchange. Consuming structured samples triggers the outgoing criteria (line 2 in Algorithm 3.12) and a set of incoming criteria results in providing outgoing samples to the agent from which these criteria are received (line 8 and 9 in Algorithm 3.12). Receiving incoming samples completes the coordination by sending a set of structuring criteria to the structuring level (line 5 and 6 in Algorithm 3.12). Finally, the organizational criteria adapt the organized samples (line 11 in Algorithm 3.12) by parameterizing the operation of an overlay service.

Algorithm 3.13 illustrates the adapt task. The adapt task performs the parameterization of the coordination level as defined in the organizational criteria (line 2 in Algorithm 3.13). It also handles the coordination by (i) consuming the incoming samples contained in a set of received incoming criteria, (ii) configuring the structuring level with the structuring criteria and (iii) generating the outgoing samples that is sent back to the initiator agent of the coordination (line 4-9 in Algorithm 3.13).

The consume task, illustrated in Algorithm 3.14, (i) initiates the coordination by generating outgoing criteria (line 2 in Algorithm 3.14) and (ii) organizes the organized samples provided to the application (line 5 in Algorithm 3.14). This task results in the 

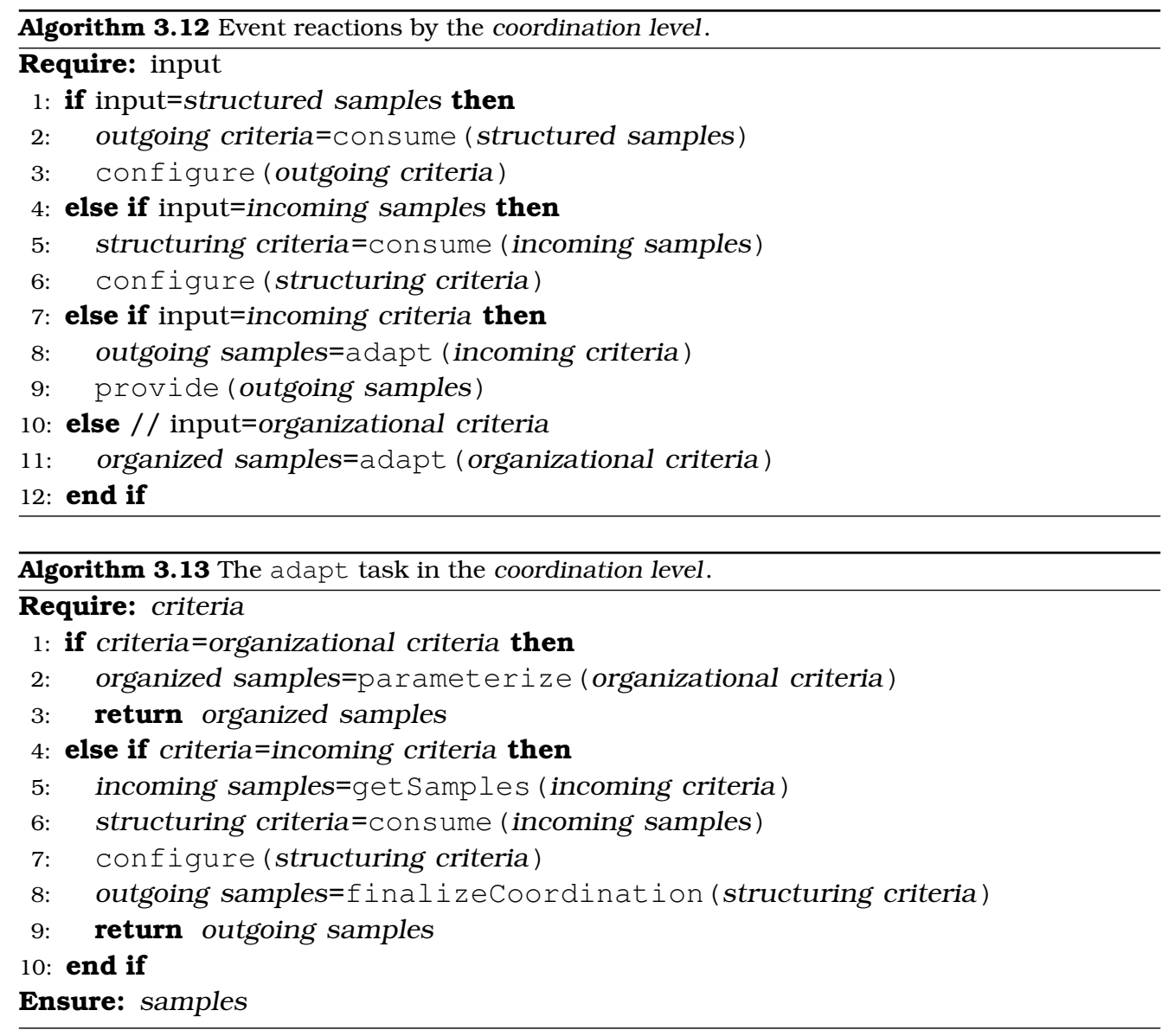

structuring criteria provided to the structuring level as feedback for the improvement of the quality of an overlay service.

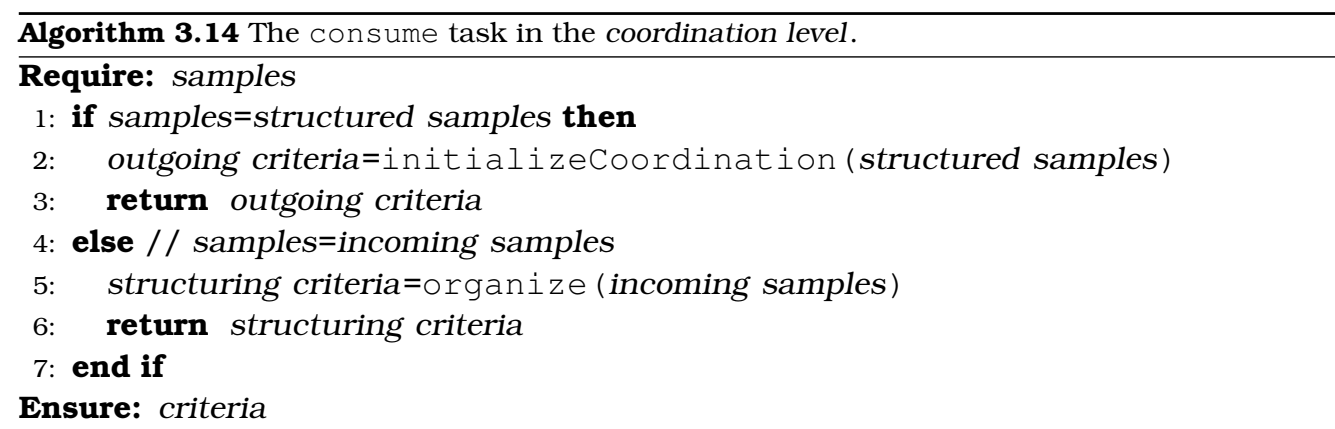

The adapt and consume tasks show that coordination is an actual response of outgoing samples to a set of incoming criteria based on the structuring criteria (line 8 in Algorithm 3.13) and structured samples (line 2 in Algorithm 3.14) respectively. Note that the initializeCoordination, finalizeCoordination, organize 
and parameterize subtasks are realized within an overlay service.

\subsection{Architecture Coupling with Applications}

An overlay service can be used by one or more distributed applications and an application instance can be composed by one or more overlay services. Based on this concept, this section illustrates how applications can utilize overlay services of ASMA. A distributed application is modeled in two levels: (i) the utilization level that facilitates the broader context of an application and (ii) the application level that facilitates the actual application. Both levels are application-dependent and exchange application samples and application criteria. Figure 3.3 illustrates this two-level application modeling.

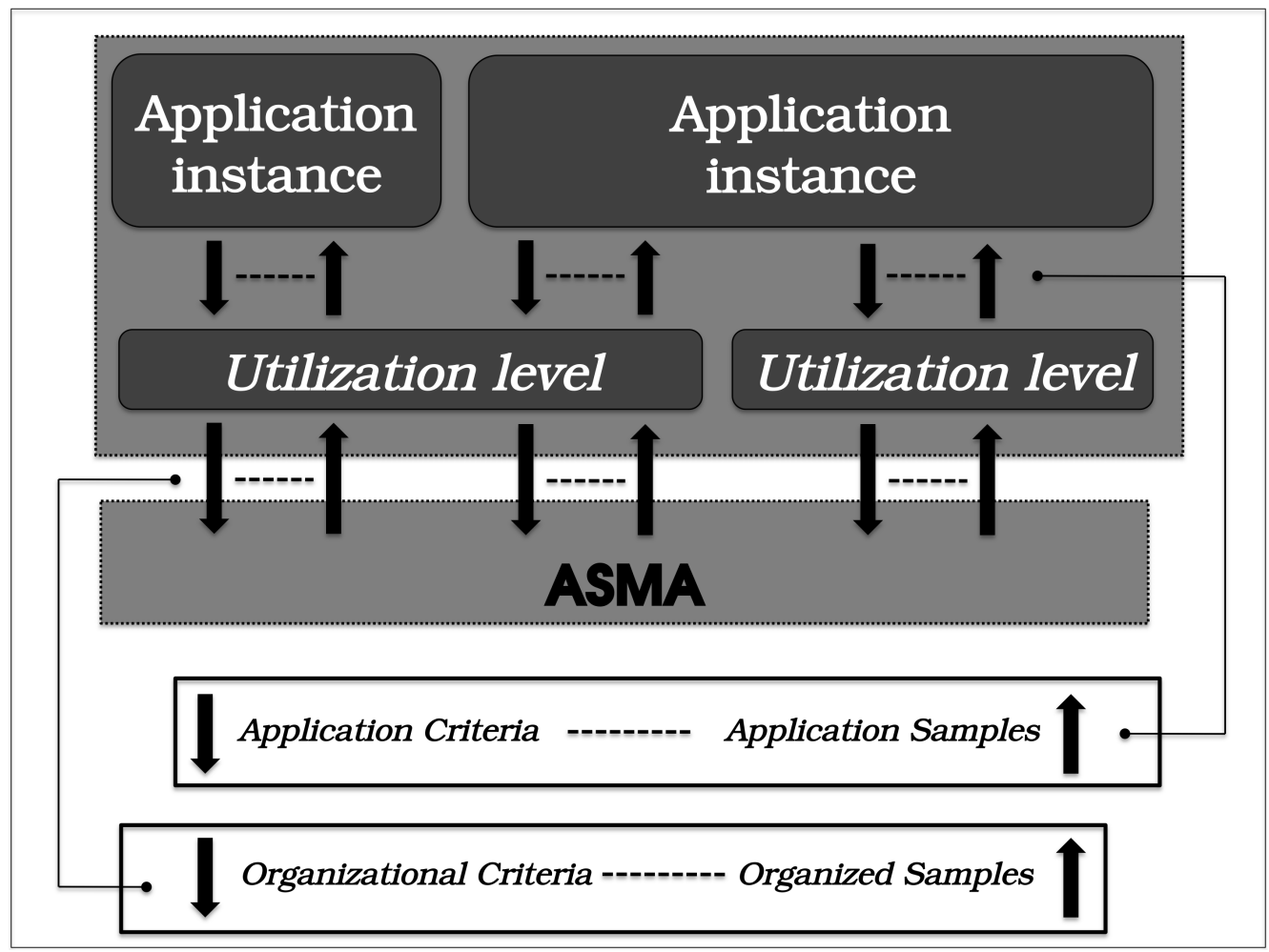

Figure 3.3: A two-level utilization of overlay services by distributed applications.

The utilization level is responsible for providing a context within which an overlay service is utilized. This context can be relevant for more than one application. Furthermore, the utilization level is responsible for the abstraction of application requirements to service requirements contained in the organizational criteria. Similarly, the organized samples are specialized to application samples that an application instance acquires for its operation. Therefore, the utilization level couples the ASMA architecture with distributed applications.

The application level contains the domain-specific logic of the application. It benefits from this two-level split of concerns as a distributed application can be actually 
composed by different utilization levels.

Finally, note that matching domain-specific and application-dependent requirements to the organizational criteria is challenging. Even more challenging is the specialization of organized samples to application samples that meet the application objectives. The effectiveness of this abstraction and specialization depends on how flexible and generic an overlay service is realized within the ASMA architecture. The multi-level architecture of ASMA is designed to support this flexibility.

\subsection{Architectural Realization}

This section introduces two overlay services designed within the ASMA architecture and illustrated in the rest of this thesis:

- AETOS, the Adaptive Epidemic Tree Overlay Service (Chapter 4).

- DIAS, the Dynamic Intelligent Aggregation Service (Chapter 5).

AETOS is a self-organization and self-optimization overlay service for overlay networks organized in various tree topologies. Trees are used for several operations such as decision-making, aggregation, information dissemination etc., with an applicability in a wide range of distributed applications, e.g., distributed databases [González-Beltrán et al., 2008, Zhuge and Feng, 2008] and multimedia multicasting [Tan et al., 2005a]. The three levels of the ASMA architecture are appropriate to model the complexity of such an overlay service: (i) discovery for accessing every possible peer in the overlay network, (ii) structuring/selecting candidate parents and children according to the target topology to be built and finally (iii) coordination between the parents and children to form bidirectional links. Distributed applications focus entirely on the utilization of a tree topology, leaving the building and maintenance to the AETOS overlay service.

DIAS computes aggregates, such as AVERAGE, SUMMATION, MAXIMUM, and STANDARD DEVIATION, of dynamically changing values distributed in every peer of an unstructured overlay network. Decentralized computation of aggregates benefits a wide range of distributed applications as this information is used for load-balancing [Pournaras et al., 2008a], data mining [Van Renesse et al., 2003], sensor networks [Boulis et al., 2003] etc. The three-level self-organization of the ASMA architecture supports the computation of continuously updated and duplicate-free aggregates: (i) discovery for accessing the information of every possible peer, (ii) structuring this information as exploited (duplicate information), unexploited (new information) or updated (changed information) and (iii) using this information for coordinating an accurate computation of aggregates between the peers of an unstructured overlay network. In this way, distributed applications are able to have a global view of various system-wide metrics, leaving the computations and update of aggregate information entirely to the DIAS overlay service.

This thesis studies these two overlay services as a proof of concept for the generic design of the ASMA architecture.

\subsection{Comparison with Related Work}

Grace et al. [2004, 2008] introduce the idea of 'open overlays' supported by a generic framework for overlay networks and their applications. This framework receives plug- 
in overlays defined by three components: 'Forwarding', 'state' and 'control'. These components are, in some aspects, similar to the three levels of ASMA. Applications are also introduced as plug-ins and are associated with possible overlay plug-ins that can support them. A plug-in can be positioned in the framework as independent or stacked with other plug-ins. Similarly with ASMA, top-down configurations are applied during deployment starting from the application plug-ins to the lower level network plug-ins. However, the number of possible combinations defined by the top-down configurations between the available overlay plug-ins can be large resulting in complex compositions.

Although dealing with overlay plug-ins is a generic, extensible and highly modular approach, Grace et al. [2004, 2008] identify that the development of the three overlay components may be blended and cannot always be intuitive enough. Furthermore, the framework of overlay plug-ins does not define any high-level semantic of the component interactions. In contrast, ASMA provides a narrower defined context and objective for every self-organization level. It also shows how these objectives are mutually supported via the exchange of criteria and samples.

Some related work focuses on supporting multiple overlays network capabilities as an IP-layer solution instead of a middleware solution that ASMA proposes for its realization. Joseph et al. [2006] and Mao et al. [2008] illustrate two representative mechanisms of this approach. OCALA [Joseph et al., 2006] positions the 'overlay convergence' layer, built by an overlay-independent and an overlay-dependent component, under the transport layer. These layers provide a level of routing and lookup transparency between physical machines belonging to different overlay networks. However, there is a plethora of problems and open issues related to the support of existing IPbased applications, security, efficiency and access to overlay functions beyond routing. MOSAIC [Mao et al., 2008] is a declarative methodology for the composition of 'horizontal' (bridged via gateways) or 'vertical' (layered similarly to ASMA) overlay networks. Although this methodology provides a highly configurable and reasoning compositional environment for overlay networks, a large amount of information must be known a priori for each individual peer of the network. In addition, MOSAIC is highly dependent on a directory service that supports the composition process. It is unclear how changes to the directory service can be automatically reflected in the composed overlay networks. Note that MOSAIC also faces the restrictions of an IP-layer solution similarly with OCALA. These approaches could in theory function complementary to ASMA overlay services for supporting communication between heterogeneous networks, e.g., wireless and wired networks.

iOverlay [Li et al., 2004] provides an interface for building overlay networks and their applications. This interface is rather limited as it only supports overlay communication leaving excessive freedom to the developer. In comparison with ASMA, the main functionality of iOverlay corresponds to the discovery level of ASMA. A similar approach with iOverlay is followed by MACEDON [Rodriguez et al., 2004]. Opus [Braynard et al., 2002] is based on a backbone service to optimize the resource allocation for different applications. Therefore, the scope of this approach is limited compared to ASMA and the other approaches illustrated in this section. Joita et al. [2005] illustrate a multilevel economic framework for Grid services and resource allocation. Self-organization is engaged for the discovery of agents that negotiate for resources. An overlay abstraction is provided to the agents. The system is designed based on web service technologies and therefore some of its components remain centralized. In contrast, ASMA introduces multiple self-organization levels for the discovery, structuring and coordination 
of samples without centralized components.

A comparison of the ASMA overlay services with related approaches is illustrated within the context of these overlay services in Section 4.9 and 5.10.

\subsection{Discussion}

The multi-level self-organization approach of the ASMA architecture addresses the challenge of abstraction in overlay services. Discovery, structuring and coordination are the components that support self-organization. The architecture of ASMA provides a split of concerns that shows how to design more generic, modular and reconfigurable overlay services. Furthermore, the continuous exchange of criteria and samples in a top-down and bottom-up fashion respectively forms inter-level feedback loops that enable dynamic adaptation and more specifically the optimization of quality of an overlay service.

Self-organization in ASMA enables different overlay services to operate in largescale decentralized environments. Furthermore, self-organization supports more informative and structured interactions between agents with semantic information. Selforganization makes overlay services customizable and flexible to perform topology adaptations according to certain application objectives. Complex distributed systems and their applications that are not based on any form of self-organization lack the flexibility required to handle frequent system changes.

The proposed utilization of overlay services designed according to the ASMA architecture provides a new compositional environment for applications. Building applications as a collection of utilization levels results in more modular and extensible applications. Furthermore, application requirements and objectives can be abstracted to applicationindependent parameters contained in the organizational criteria via the utilization level. All these motivate a more modular and incremental development cycle of applications based on overlay services of ASMA.

Making the best out of this generic approach requires a degree of domain or application awareness: an overlay service can be used by different distributed applications and an application can be composed by different overlay services. Note that this thesis does not aim to prove that distributed applications perform better or overlay services achieve higher quality when using ASMA. The ASMA architecture motivates a higher abstraction, modularity and reconfigurability for building overlay services. However, introducing multiple abstraction levels may influence performance. This trade-off is studied in the overlay service realizations of ASMA.

\subsection{Conclusions}

The management of distributed applications with an increasing number of integrated features supported by overlay networks is challenging. Moving some of this complexity in a middleware architecture is required. This is an issue discussed in literature [Braynard et al., 2002, Grace et al., 2004, 2008, Joita et al., 2005, Joseph et al., 2006, Li et al., 2004, Mao et al., 2008, Rodriguez et al., 2004]. ASMA is a conceptual selforganization architecture that is able to incorporate some of this complexity and more specifically, the continuous and reconfigurable discovery, structuring and coordination 
of system entities.

The next two chapters introduce two complex overlay services whose design and implementation adopts the ASMA architecture. 


\title{
Chapter 4

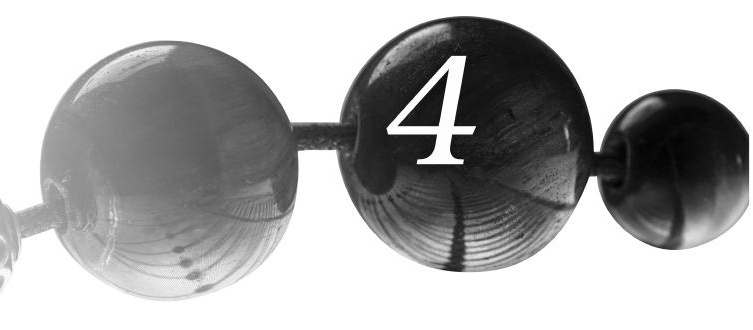

\section{The Adaptive Epidemic Tree Overlay Service*}

\author{
"Opposition brings concord. Out of discord comes the fairest \\ harmony."
}

Heraclitus $^{1}$

Distributed systems and their applications often require an organizational structure to perform their operations efficiently. Certain topologies of overlay networks are defined by graph properties that enable or enhance a specific application. Tree topologies are one of them. Their properties, e.g., path uniqueness, and the hierarchy they reflect enable operations such as decentralized search, decision-making, aggregation or information dissemination. These operations are fundamental in various application domains such as distributed databases [González-Beltrán et al., 2008, Zhuge and Feng, 2008], application-level multimedia multicasting [Tan et al., 2005a] and grid computing [Chakravarti et al., 2006].

However, trees suffer from lack of redundancy and, therefore, their structure is very sensitive to single node or link failures. Moreover, a tree experiences heterogeneous load, meaning that nodes close to the root of the topology receive messages forwarded from all other nodes at the bottom part of this tree. This effect is also related to the impact of a failure that is much higher for nodes close to the root of a tree. All of these issues make the use of trees in dynamic distributed environments challenging and sometimes infeasible.

Self-organization in trees is required to overcome these limitations. A robust tree requires both building and maintenance during runtime of a distributed application. Nodes are self-organized in a tree topology that ideally minimizes the impact of their failures. One way to achieve this optimization is by ordering a tree according to specific application criteria, i.e., the performance profile of nodes. An optimized tree may also have constraints such as the number of children with which each node can connect.

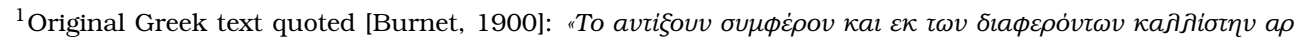

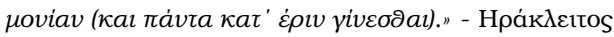

*This chapter is based on three published papers [Pournaras et al., 2010b,a, 2009a]. 
All of these criteria should be met during self-organization. However, because these criteria may vary significantly between different distributed applications that utilize tree topologies, creating a generic overlay service for building a wide range of tree topologies is the challenge this chapter addresses. This overlay service is AETOS, the Adaptive Epidemic Tree Overlay Service.

Self-organization in AETOS is based on the following approach: (i) Discovering candidate parents and children in the network, (ii) structuring these nodes based on their proximity information related to application criteria and (iii) coordinating tree building and maintenance by establishing bidirectional parent-child links. The conceptual architecture of ASMA, the Adaptive Self-organization in a Multi-level Architecture, introduced in Chapter 3, provides the design of this approach. AETOS is an overlay service realization of ASMA. This chapter also introduces a number of performance metrics used as an expression of the quality of the AETOS overlay service.

A number of adaptation strategies within the structuring level provide abstraction, modularity and customization in the self-organization of trees. Tree topologies with different graph properties are formed by simply adopting adaptation strategies. The complexity of adaptation strategies is hidden from the coordination level and they are applied in an automated fashion during self-organization. Adaptation strategies can be combined dynamically, providing a powerful meta-level of adaptation and abstraction in the self-organization of tree topologies based on which a wide range of performance trade-offs can be explored as confirmed by the experimental findings of this chapter.

The organization of this chapter is outlined as follows: Section 4.1 illustrates the graph properties of tree topologies and their relation with distributed applications. It also defines the organizational goal. Section 4.2 provides a high-level overview of AETOS. Section 4.3 illustrates the discovery level of AETOS. Section 4.4 discusses the input and adaptations of the structuring level of AETOS. Section 4.5 shows the building and maintenance process of trees in the coordination level. Moreover, Section 4.6 introduces a number of generic adaptation strategies engaged in the structuring level of AETOS. Section 4.7 illustrates an experimental evaluation of AETOS and Section 4.8 summarizes the experimental results. Section 4.9 compares AETOS with related methodologies. Section 4.10 discusses various aspects of AETOS and outlines future work. Finally, Section 4.11 concludes this chapter.

\subsection{Problem Description}

This chapter illustrates the problem of designing a generic self-organization overlay service for building and maintaining tree topologies defined by various graph properties. Trees are connected undirected acyclic graphs [Baldwin and Scragg, 2004, Gross and Yellen, 2005] with some graph properties such as degree-bounding, ordering, balancing and completeness that influence the performance of distributed applications, making a tree more robust to node failures or balancing the load between nodes. This chapter focuses on building optimized tree topologies that tune and reflect specific graph properties to meet requirements of various application types.

This section illustrates these graph properties and their relation to various distributed applications. It also defines the organizational goal for a certain tree topology that (optimally) reflects the illustrated graph properties, referred to as the optimum topology for the given properties. For illustration purposes, the organizational goal is 
explained as a centralized algorithm. A number of application-independent performance metrics is introduced for the evaluation of this goal. These metrics quantify the quality of the incremental self-organization process.

\subsubsection{Graph properties and applications}

A tree topology can be represented as a connected undirected acyclic graph with its name denoting its properties [Baldwin and Scragg, 2004, Gross and Yellen, 2005]:

- Connected: A graph is connected if there is at least one path between any two vertices ${ }^{2}$. A disconnection of a node from a tree may have significant impact as the graph is split in two branches forming a type of graph called a forest. The impact of disconnection and the new structure of the graph depends on the position of the disconnected node and specifically if this node is placed close to the root or close to the leaves. A distributed application based on a connected overlay network is able to access all of the available information in nodes. Disconnected overlay networks do not perform well as network resources remain unexploited in multiple disconnected clusters or components as referred in graph theory. For example, a multimedia multicasting application is disrupted [Frey and Murphy, 2008] if a tree is disconnected and, therefore, content cannot be forwarded further. Similarly, aggregation queries over a disconnected tree result in inaccurate estimations of aggregates [Li et al., 2006, Ogston and Jarvis, 2010]. There is a significant research effort on maintaining dynamic and connected overlay networks using, for example, gossiping protocols as studied by Jelasity et al. [2007].

- Undirected: A graph is undirected if its edges ${ }^{3}$ do not have a direction. An undirected link between two nodes of an overlay network denotes that communication is permitted in both ways between these nodes. Establishment of an undirected link in an overlay network is more complex than a directed one as this may be subject of mutual confirmation or negotiation between two nodes. Furthermore, failures need to be explicitly detected via, for example, heartbeat mechanisms [Yang and Fei, 2007]. An undirected tree allows top-down and bottom-up communication: from the root to the leaves and from the leaves to the root respectively.

- Acyclic: A graph is acyclic if the edges of its vertices do not form cycles. In such a graph, there is a unique path between any two vertices. Path uniqueness can be critical for distributed applications as it guarantees that the information exchanged between the nodes of a tree overlay network is not duplicated. Furthermore, path uniqueness provides a lower communication overhead as redundant communication is prevented. For example, multimedia multicasting applications [Tan et al., 2005a] aim for efficient dissemination of multimedia content. Efficiency is achieved by maximizing delivery speed and minimizing the delay experienced in a network. One possible way to meet these requirements is by acquiring unique paths to reach all of the nodes of a network efficiently. Path uniqueness increases delivery speed as nodes receive multimedia content only once. Therefore, bandwidth consumption for each node is lower and more efficient. However, path uniqueness does not come without drawbacks in overlay

\footnotetext{
${ }^{2}$ Vertices, as usually referred to in graph theory, correspond to the nodes of an overlay network topology

${ }^{3}$ Edges, as usually referred to in graph theory, correspond to the links of an overlay network topology.
} 
networks. A tree topology cannot easily tolerate arbitrary failures of nodes due to the lack of path redundancy between nodes.

Figure 4.1 illustrates an example of transforming a disconnected directed cyclic graph to a tree that is a connected undirected acyclic graph.

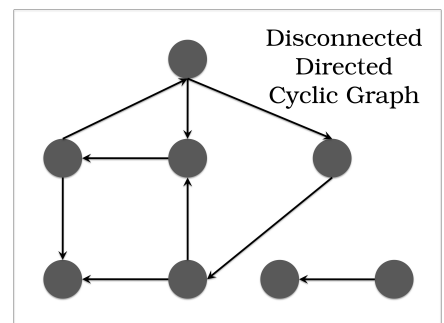

(a) Starting from a disconnected directed cyclic graph.

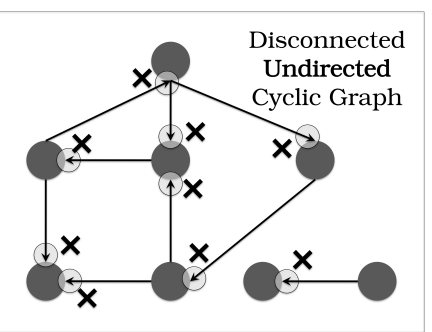

(b) Removing the direction from all the edges of the graph. The graph becomes undirected.

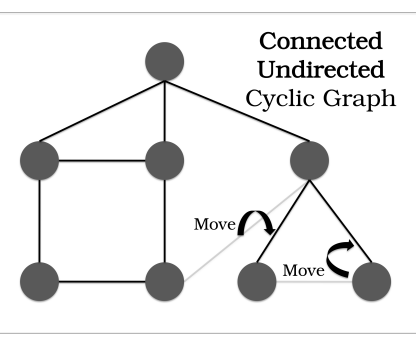

(c) Moving one or more edges to connect the disconnected component of the graph. Graph becomes connected

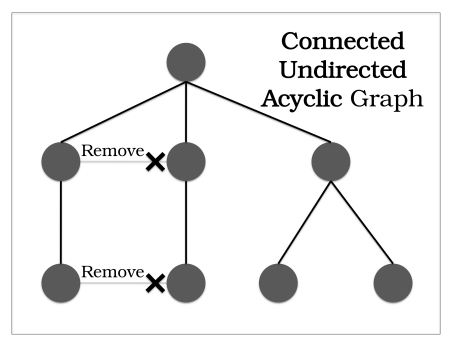

(d) Removing edges that create cycles. Graph becomes acyclic.

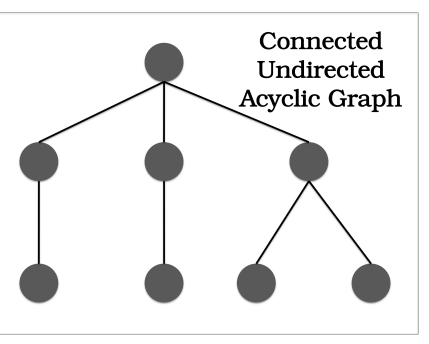

(e) The resulting tree.

Figure 4.1: An example of forming a connected undirected acyclic graph (tree).

The trees referred to in this chapter are rooted trees, meaning trees that have a distinguished root [Gross and Yellen, 2005]. The height of a rooted tree is the length of the longest path from the root to the leaves. Similarly, every vertex in a tree graph has a depth. Selecting and studying graph properties of networks is a challenging problem as illustrated by Li et al. [2011]. This chapter focuses on trees with specific graph properties that influence the performance of several distributed applications. These properties and their relation to applications are the following:

- Degree-bounding: The degree of a vertex is the number of (non-directional) edges attached to this vertex. The degree of a vertex that belongs to a tree is the number of its children plus one for its parent. Degree-bounding of a tree topology refers to the maximum number of children that a node supports and is related to various application constraints. For instance, bandwidth, storage and processing capacity of a node are related to the maximum number of nodes to which multimedia content can be disseminated [Fei and Yang, 2007]. Degree-bounding should be respected during building and maintenance of tree topologies. If the degree of a node is much lower than the upper bound, then the node is underloaded or the resources allocated for its application are not fully exploited. In contrast, if the 
degree of a node exceeds the upper bound, then the node is overloaded resulting in a bottleneck or unavailability of this node. In this case, the topology may disconnect and the performance of the application degrades.

- Ordering: An ordered tree is a rooted tree in which the children of each vertex are assigned a fixed ordering. This ordering is performed according to assigned weights that rank vertices of a tree. These weights are usually related to an application. For example, multimedia multicasting applications [Birrer and Bustamante, 2007] compute the bandwidth and availability [Bhagwan et al., 2003] of nodes. The latter is usually related to the uptime of the nodes in the network. Information about these metrics can be used for the maximization of content delivery and speed that is crucial for real-time applications such as multimedia multicasting. Similarly, social recommender systems [Manouselis and Costopoulou, 2007] capture the profiles of users based on their preferences about an online product or service. Based on preference matching, these systems aim to increase engagement of users and the quality of recommendations.

There are different ordering schemes that can be applied to a tree such as the levelorder, pre-order, post-order and in-order [Gross and Yellen, 2005]. This chapter focuses on the level-order scheme which is described as a top-down and left-toright traversal. However, note that in practice the actual communication between the nodes always occurs via the parent-children links. Because of this fact, the distributed applications to which this chapter refers to mainly require a top-down order rather than a left-to-right order.

Ordering can make a tree used by a specific distributed application more robust. For example, positioning nodes with high availability [Bhagwan et al., 2003] close to the root instead of nodes with low availability, reduces the impact of a node failure on the tree as the size of disconnected branches is smaller on average. Similarly, node close to the root of a tree with low bandwidth is a bottleneck for multimedia multicasting applications. Therefore, ordering provides a proactive robustness by minimizing the impact that a low-performing node causes to the topology and its distributed applications.

- Balancing: A balanced tree with a degree-bounding for each vertex is a rooted tree from which the leaves have the same depth or their depth difference is ' 1 ', if degreebounding of the vertices does not permit having the same depth. An imbalanced tree experiences an unequal load among different branches with different path lengths. Therefore, distributed applications may experience higher latency and communication overhead due to the higher number of hops to reach each node of a tree [Jagadish et al., 2006a].

- Completeness: A complete tree is a rooted tree that is balanced and all its vertices, except the leaves, have the maximum possible number of children as defined by the degree-bounding. Complete trees have the minimum possible height given the degree-bounding of the vertices. Overlay networks organized in complete tree topologies make effective resource allocation for their applications as nodes exploit the maximum number of children without exceeding the upper bound of the node degrees [Wang et al., 2010]. 
Figure 4.2 illustrates an example of transforming an any-ary unordered imbalanced incomplete tree to a 2-ary (binary) ordered balanced complete tree. This chapter addresses the challenge of building and maintaining trees that reflect these properties in a dynamic distributed environment.

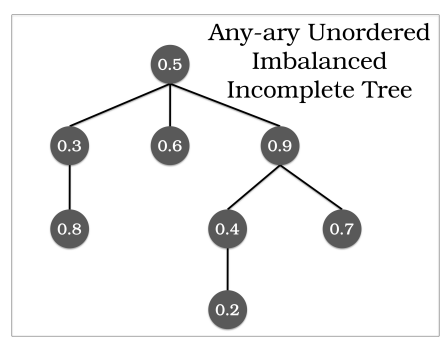

(a) Starting from an any-ary unordered imbalanced incomplete tree.

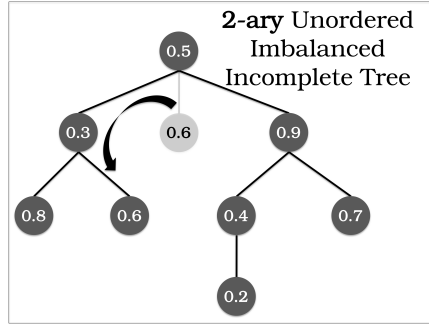

(b) Moving a third child connected to the root at a lower level. Tree becomes 2-ary (binary).

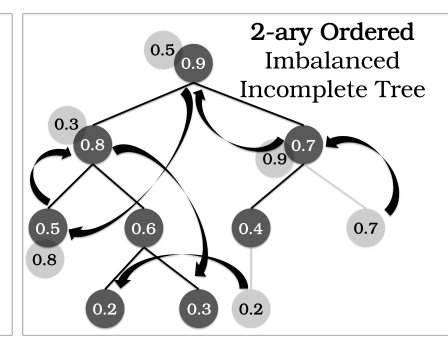

(c) Positioning the higher ranked nodes close to the root and the lower ranked ones as leaves. Tree becomes ordered. Each node has a higher ranked parent, lower ranked children and respects the degree-bounds $(\leq$ 2).

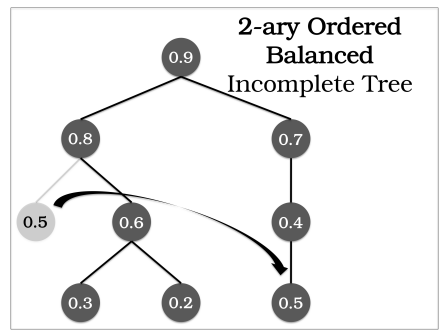

(d) Moving a node at a lower level to make the tree balanced.

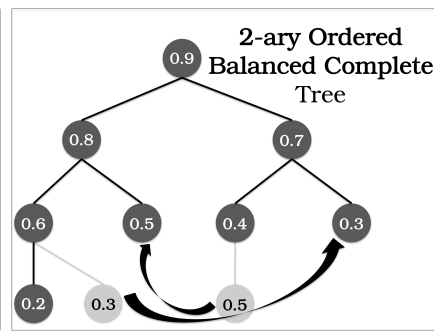

(e) Moving two nodes at a higher level to make the tree complete.

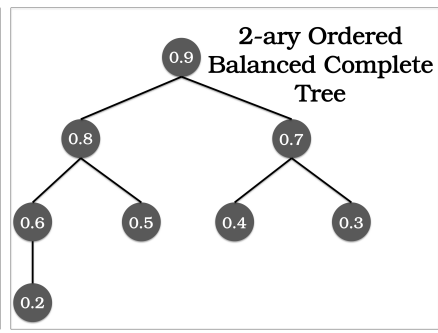

(f) The resulting tree.

Figure 4.2: An example of forming a 2-ary (binary) ordered balanced complete tree.

\subsubsection{Organizational goal}

The properties described in Section 4.1.1 form the building blocks of the organizational goal of this chapter that is formalized as follows:

Assume an overlay network of $n$ nodes that forms a tree topology. Each node $i$ is ranked with a unique ${ }^{4}$ weight $w_{i}$ in the range $[0,1)$ that represents one or more application criteria. Furthermore, each node $i$ has a node degree $d_{i}$ that refers to the maximum number of links that this node can establish. Similarly, the maximum number of children that a node $i$ has is $k_{i} \leq d_{i}-1$. Finally, each node $i$ has a tree view $\mathbf{v}_{i}$ (tree) that is a list of other ranked nodes. The first element of a tree view corresponds to the parent and the rest of the elements to the children of node $i$.

Next, assume an ordered list of ranked nodes $\mathbf{w}=\left\{w_{0}, \ldots, w_{n-1}\right\}$ under the '>' relation with the respective number of children $\mathbf{k}=\left\{k_{0}, \ldots, k_{n-1}\right\}$. Each size $k_{i} \in \mathbf{k}$ corresponds

\footnotetext{
${ }^{4}$ The requirement for a rank uniqueness can be technically met with a randomization scheme.
} 
to the ranked node $w_{i} \in \mathbf{w}$. An ordered tree $\mathbf{T}=\left\{\mathbf{w}_{0}, \ldots, \mathbf{w}_{h-1}\right\}$ can be built by splitting the ordered list $\mathbf{w}$ in $h$ number of levels $w_{0}, \ldots, w_{h-1}$. An indexed level $u-1$ of parents $\mathbf{w}_{u-1}=\left\{\mathbf{w}_{p_{\vdash}}, \ldots, \mathbf{w}_{p_{t}}\right\}$ is defined by the list of the lowest ranked node $w_{p_{\vdash}} \in \mathbf{w}$ and the highest ranked node $w_{p_{\uparrow}} \in \mathbf{W}$ in this level respectively. Similarly, the lowest ranked child $w_{c_{\vdash}} \in \mathbf{w}$ and the highest ranked child $w_{c_{\uparrow}} \in \mathbf{w}$ form the list of ranked children $\mathbf{w}_{u}=\left\{\mathbf{w}_{c_{\vdash}}, \ldots, \mathbf{w}_{c_{\uparrow}}\right\}$ that builds the next level with size $\left|\mathbf{w}_{u}\right|=g_{u}$.

A degree-bounded, ordered, balanced and complete tree can be built in a centralized fashion according to Algorithm 4.1. The tree is built level-by-level using a buffer of parents $p_{\vdash}, \ldots, p_{\dashv}$ and children $c_{\vdash}, \ldots, c_{\dashv}$ indexing nodes in the ordered list $\mathbf{w}$. The number of children that the next level includes is computed at the beginning (line 4-8 of Algorithm 4.1). Next, every indexed node from the level of parents is matched with the indexed nodes from the level of children (line 9-19 of Algorithm 4.1). Therefore, the tree views can be filled using these indices. This process repeats by incrementing the level of parents to level of children and recomputing the next level of children at the next iteration (line 21 of Algorithm 4.1). Figure 4.3 visualizes this incremental process.

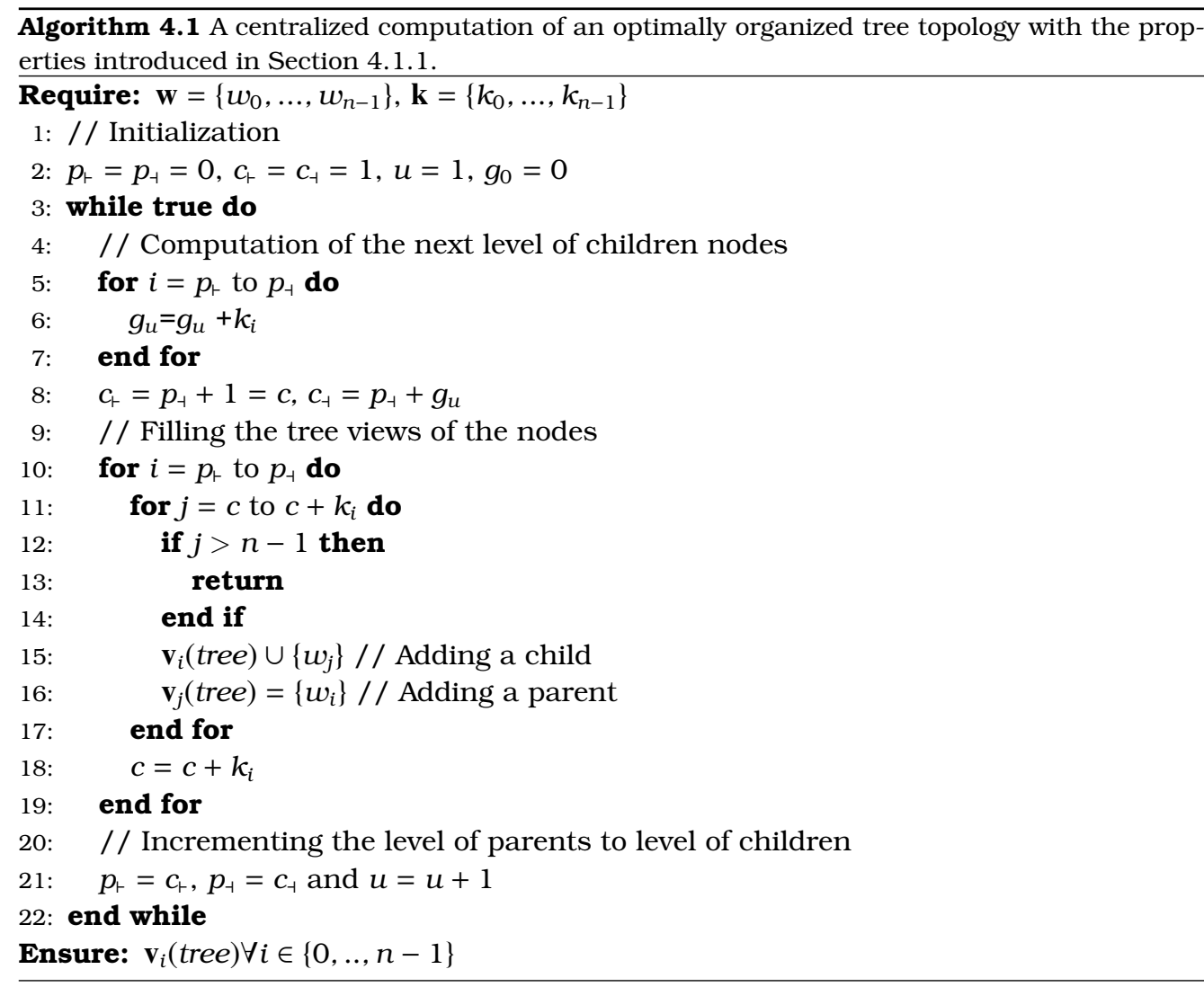

The above tree topology is optimally and centrally organized as the whole set of ranked nodes is known. However, in a decentralized self-organization, agents have limited information about other agents and therefore, they need a certain execution time to discover, structure and coordinate with other agents with which they form parent-child links. Performance metrics are required to quantify the degree to which 


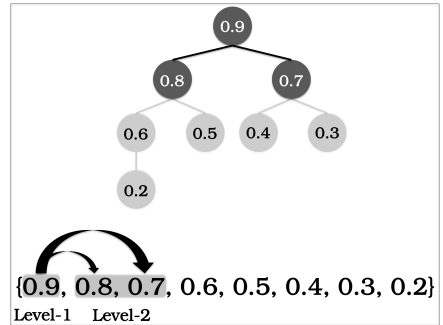

(a) Level-2.

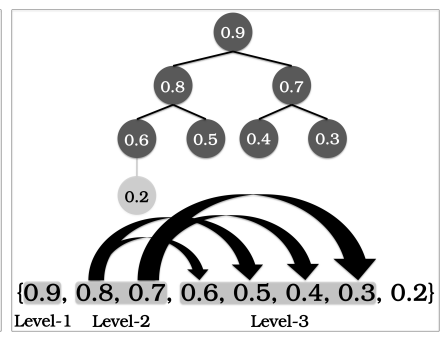

(b) Level-3.

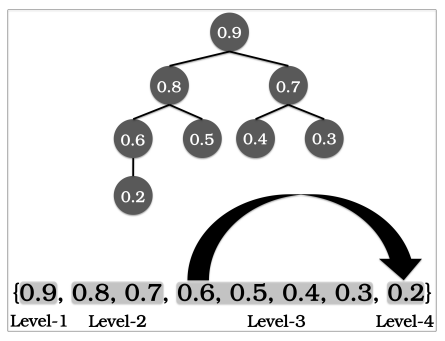

(c) Level-4.

Figure 4.3: Centralized building of a binary, ordered, balanced, and complete tree by defining the tree levels incrementally.

the illustrated graph properties are met during the self-organization runtime. This chapter introduces four performance metrics for this purpose: (i) connectedness $\beta$, (ii) connectivity $\gamma$, (iii) fitness $\rho$ and (iv) instability $\delta($ ).

Connectedness $\beta$ is the proportion of nodes that are connected to the largest component of the forest: the main tree. For a forest $\mathbf{F}$ of $n$ tree components $\left\{\mathbf{T}_{0}, \ldots, \mathbf{T}_{n-1}\right\}$, connectedness is defined as:

$$
\beta=\frac{\max \left(\left|\mathbf{T}_{0}\right|, \ldots,\left|\mathbf{T}_{\mathrm{n}-1}\right|\right)}{n}
$$

In contrast, the tree connectivity $\gamma$ refers to the number of links established between the nodes relative to the maximum number of links $n-1$ that can be established in a tree topology [Gross and Yellen, 2005]:

$$
\gamma=\frac{1}{2(n-1)} \sum_{i=0}^{n-1} \mid \mathbf{v}_{i}(\text { tree }) \mid
$$

Note that the computation of connectedness is mainly related to the nodes of a tree, whereas, connectivity is associated with the links. This is an intuition for readers to distinguish their difference.

The fitness $\rho$ expresses the quality of the ordering process based on the selection of the parent and children for each node. Fitness is associated with the ranking of nodes that form parent-child links. This chapter correlates the fitness $\rho$ with the relative ranking distance between nodes and their parents. This is the distance $w_{0} \in \mathbf{v}_{i}($ tree $)-w_{i}$ between a ranked node $w_{i}$ and its parent $w_{0} \in \mathbf{v}_{i}$ (tree) in relation to the maximum distance that they can have if the node $i$ has the highest ranked node for parent. For example, assuming nodes with uniformly distributed weights in $[0,1)$, the maximum possible ranking distance is approximately $1-w_{i}$. Therefore, fitness $\rho$ is defined as:

$$
\rho \approx \frac{1}{n \rho^{\prime}} \sum_{i=0}^{n-1} \frac{w_{0} \in \mathbf{v}_{i}(\text { tree })-w_{i}}{1-w_{i}}
$$

where $\rho^{\prime}$ is the optimum fitness computed using Algorithm 4.1.

Note that the optimum fitness $\rho^{\prime}$ depends on the topology built and therefore it is related to the other tree properties such as the degree-bounding. The maximum optimum fitness value corresponds to a tree organized in a star topology. In this case, 
every node has the highest ranked node as a parent. In contrast, the minimum optimum fitness value corresponds to a tree organized in an ordered list.

Finally, the instability $\delta()$ of a performance metric describes the variation of this metric during a self-organization runtime period. Instability is calculated using relative standard deviation. For example, instability $\delta(\rho)$ of fitness $\rho$ during a period of time $\hat{t}$ is defined as follows:

$$
\delta(\rho)=\frac{1}{\bar{\rho}} \sqrt{\frac{1}{\hat{t}-1} \sum_{\substack{t-1 \\ t=0}}^{t}[\rho(t)-\bar{\rho}]^{2}}
$$

Note that minimizing instability of a performance metric is a factor to detect the convergence of self-organization according to this performance metric.

\subsection{System Overview}

This chapter introduces AETOS, the Adaptive Epidemic Tree Overlay Service. AETOS is an overlay service that provides middleware-level self-organization capabilities in overlay networks to organize themselves in tree topologies with the properties of degreebounding, ordering, balancing and completeness illustrated in Section 4.1. Discovery, structuring and coordination are crucial operations in such an overlay service. Discovery is required to access the samples of agents such as their network address for communication and weights with which their nodes are ranked. Structuring of ranked nodes is crucial for a more informed selection of the parent and children for every node. Finally, the parent-child bidirectional links cannot be established without coordination because of the constraints that should be respected such as the degree-bounding of the nodes and the weights with which each node is ranked. These are reasons that make the levels of the ASMA architecture, illustrated in Chapter 3, relevant for the realization of the AETOS overlay service.

Figure 4.4 illustrates the architecture of AETOS realizing the three levels of the ASMA architecture. Note that this figure is not a redefinition of the architecture but a specialization of the main concepts defined in Figure 3.1. The discovery level in AETOS provides a gossip-based dissemination and collection of discovered samples corresponding to the aforementioned samples of agents. The discovery criteria are actual gossiping criteria that parameterize the discovery level and inject the weights of the nodes that participate in the tree self-organization. These samples together with the network addresses of the agents are continuously exchanged in a gossip fashion. The discovered samples are the actual agent samples provided to the structuring level that performs clustering. Agent samples are clustered based on the proximity of their weights defined by an adopted adaptation strategy. The structuring criteria contain an actual adaptation feedback and other clustering criteria that parameterize the adopted adaptation strategy. Clustering provides a search space from which candidate parents and children are selected. These are the structured samples provided to the coordination level responsible for coordinating the building and maintenance of the tree topology. The agents corresponding to the candidate parents and children are contacted for the establishment of parent-child links. If this negotiation is successful, the tree view is filled and is provided to applications as the organized samples. Finally, the organizational criteria are tree criteria containing information about the graph properties of the built tree. 


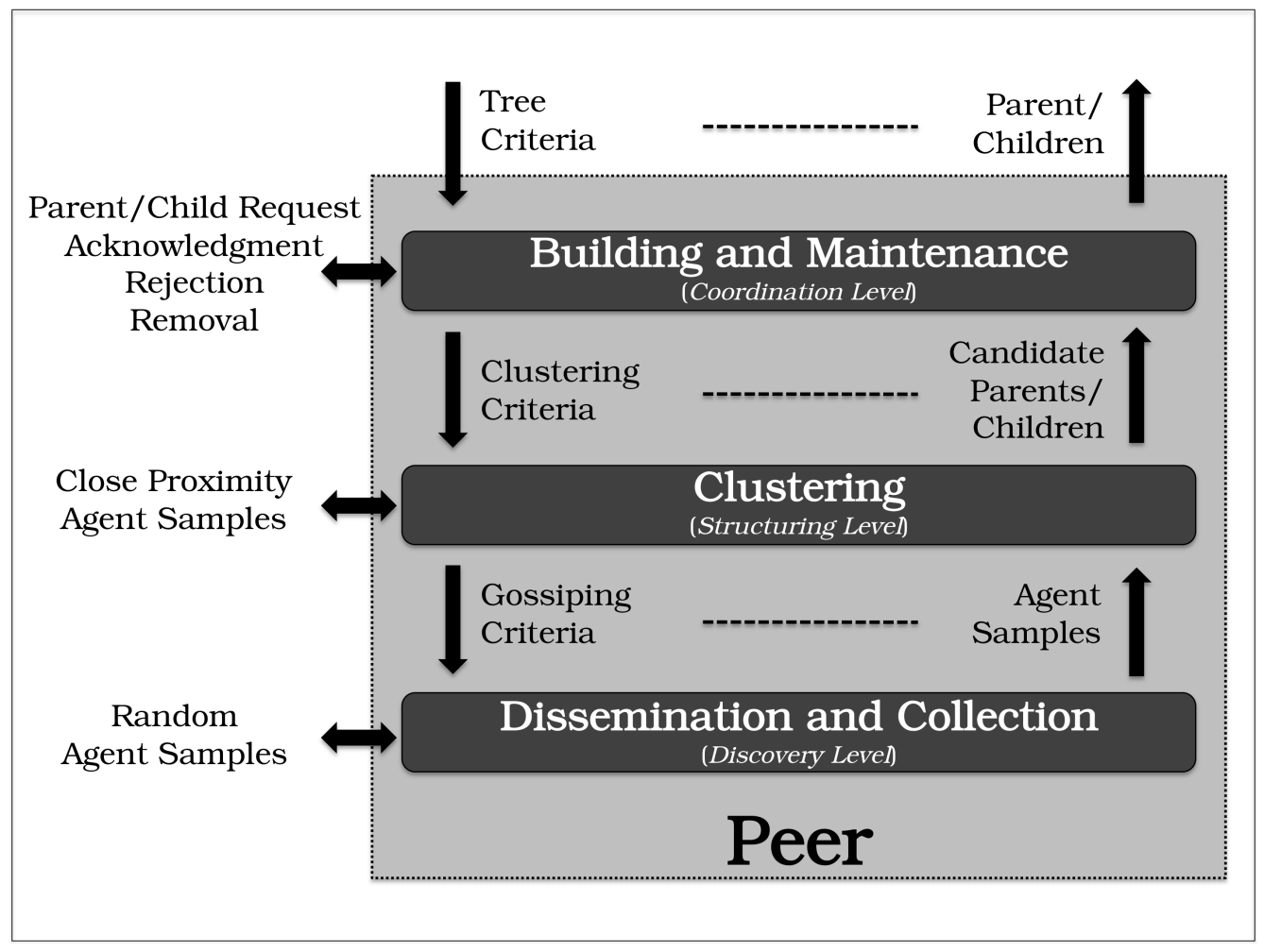

Figure 4.4: The AETOS middleware architecture. The architectural levels, criteria and samples realize the ones defined in ASMA as illustrated in Figure 3.1.

Figure 4.5 illustrates the main concept of how AETOS performs self-organization of tree topologies. Each level of AETOS contains a view containing ranked agent samples. Each view is filled incrementally by the view at the level below. Each peer of a network has three views that correspond to layered overlay networks. The bottom overlay network is defined by random samples of agents collected by the discovery level. These random discovered samples become the input at the structuring level in which they are clustered and form a clustered overlay network of candidate parents and children based on the proximity of their weights. Finally, based on a selection scheme defined by an adaptation strategy, such as the selection of the highest ranked agents, candidate parents and children potentially fill the tree view.

A more detailed illustration of the functionality of each level follows in the next sections.

\subsection{Dissemination and Collection}

The discovery level of AETOS is realized by the peer sampling service, a gossiping protocol introduced by Jelasity et al. [2007]. This gossiping protocol is illustrated in Section 3.1.2 as a possible realization of the discovery level in ASMA. The intuition of this selection is to position a highly connected and dynamic overlay network under the tree topologies 


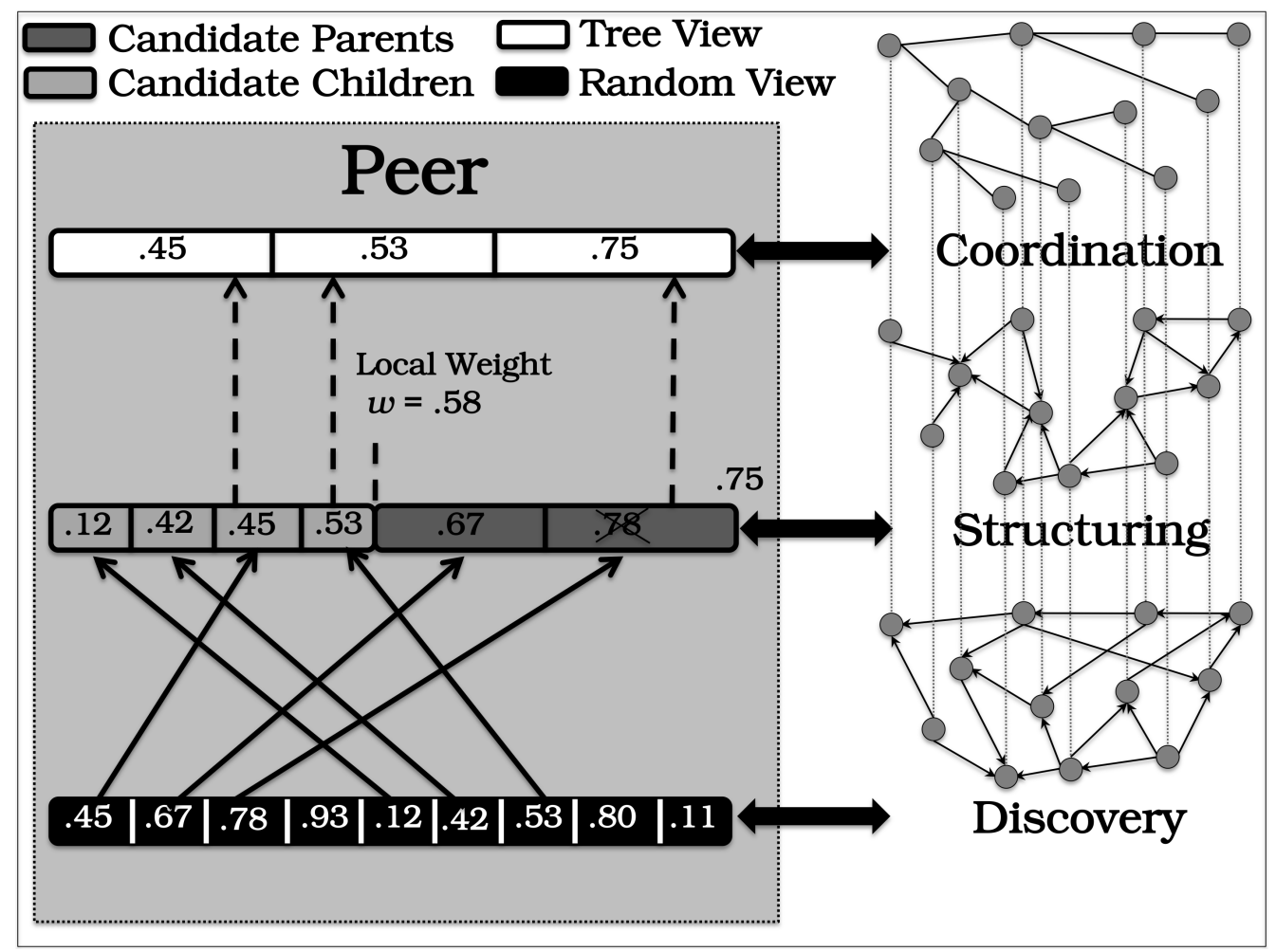

Figure 4.5: Managing the weights of agents in three levels with their views forming three layered overlay networks.

built and maintained by AETOS. This is crucial because of the sensitivity of trees in single node and link failures that can easily disconnect the topology. An underlying dynamic gossiping mechanism provides (i) a connected overlay network and (ii) the discovery of agent samples required for the self-organization of trees.

The discovery level maintains the random view that is a list of limited size $r$ containing the discovered samples. The discovered samples exchanged by the discovery level in AETOS concern the network identifiers of the respective agents located in the peers of a network, e.g., the IP address and the port number. For each unique identifier of an agent, the weight $w_{i}$ of its node $i$ is included in the discovered samples. Additional information can be included such as a timestamp of this weight or the uptime of an agent. These scenarios are discussed in Section 4.10.

\subsection{Clustering}

This section illustrates the cluster-based structuring performed in the structuring level of AETOS. This level periodically selects and provides candidate parents and children to the coordination level based on clustering criteria received by the coordination level. Event generations of the structuring level in AETOS are time-based (line 1 of Algorithm 3.7). This section shows how the input of agent samples is managed, enhanced 
and how these samples are clustered dynamically based on a number of introduced adaptation strategies.

\subsubsection{The input of the proximity view}

Searching randomly for the parent and children of each node may not be a cost-effective approach for the self-organization of tree topologies. In many cases, a metric based on a proximity distance may be more effective. The proximity distance between a ranked node $i$ and its input node $j$ provided by the discovery level is defined by their ranking distance $\left|w_{i}-w_{j}\right|$. This computation indicates the proximity of the nodes. Discovered samples are structured in an ordered list of limited size, the proximity view $\mathbf{v}_{i}$ (proximity), based on their ranking distance. Each proximity view $\mathbf{v}_{i}$ (proximity) of a node $i$ is virtually split in two parts containing candidate parents and candidate children. The weight $w_{i}$ of a node $i$ is the reference point for this split: For example, nodes ranked higher than $w_{i}$ are candidate parents and nodes ranked lower are candidate children. The maximum size of the proximity view $q_{i}$ of a node $i$ is controlled by the scaling factor $f$ and is related to the maximum number of children $k_{i}$ as follows: $q_{i}=f *\left(k_{i}+1\right)$. The scaling factor determines the number of times the proximity view is larger than the tree view.

The proximity view has random samples of ranked nodes provided by the discovery level of AETOS. The proximity view provides a smaller search space for nodes to select a parent or child in closer proximity distance. Although clustering of random nodes is possible, other methodologies can enhance the discovery speed. This section outlines the T-MAN gossiping protocol [Jelasity et al., 2009]. Its impact on the performance of AETOS is shown experimentally in Section 4.7.8. T-MAN is introduced in AETOS to improve performance, i.e., higher convergence speed in the self-organization process, by determining the proximity view with ranked nodes in closer proximity. Therefore, tree building, balancing and ordering is more efficient as defined in Section 4.1.2. Nodes in T-MAN are clustered on the basis of proximity. Agents exchange their proximity views with other agents in close proximity. T-MAN is relevant in the context of AETOS for three reasons: (i) It is a decentralized self-organization mechanism. (ii) It performs organization of nodes based on their ranking distance indicating their proximity. (iii) It requires samples of ranked nodes that the architecture of ASMA defines and AETOS supports at the discovery level. Although T-MAN is a generic topology management mechanism for building and maintaining various topologies based on the ranking distance of nodes, it is not generic enough to organize complex topologies such as the tree topologies illustrated in Section 4.1 that have organizational constraints, e.g., degree-bounding, acyclic paths during building, etc. Furthermore, T-MAN cannot guarantee a cycle-free topology during self-organization.

Algorithms 4.2 and 4.3 illustrate the T-MAN functionality embedded in the adapt and consume tasks of the structuring level. Note that the T-MAN functionality blocks replace the optional blocks indicated in Algorithm 3.9 and 3.10 respectively. Furthermore, the event generations of T-MAN are periodic, meaning that the configure (outgoing criteria) (line 4 of Algorithm 3.7) is executed every fixed period of time. The periodic executions are studied experimentally in Section 4.7.7.

The exchange of the proximity views between agents in close proximity is performed using horizontal interactions of ASMA. Outgoing criteria and outgoing samples contain the exchanged proximity views. The selectToCluster subtask selects the ranked node with which the proximity views are exchanged and embeds the proximity view in 

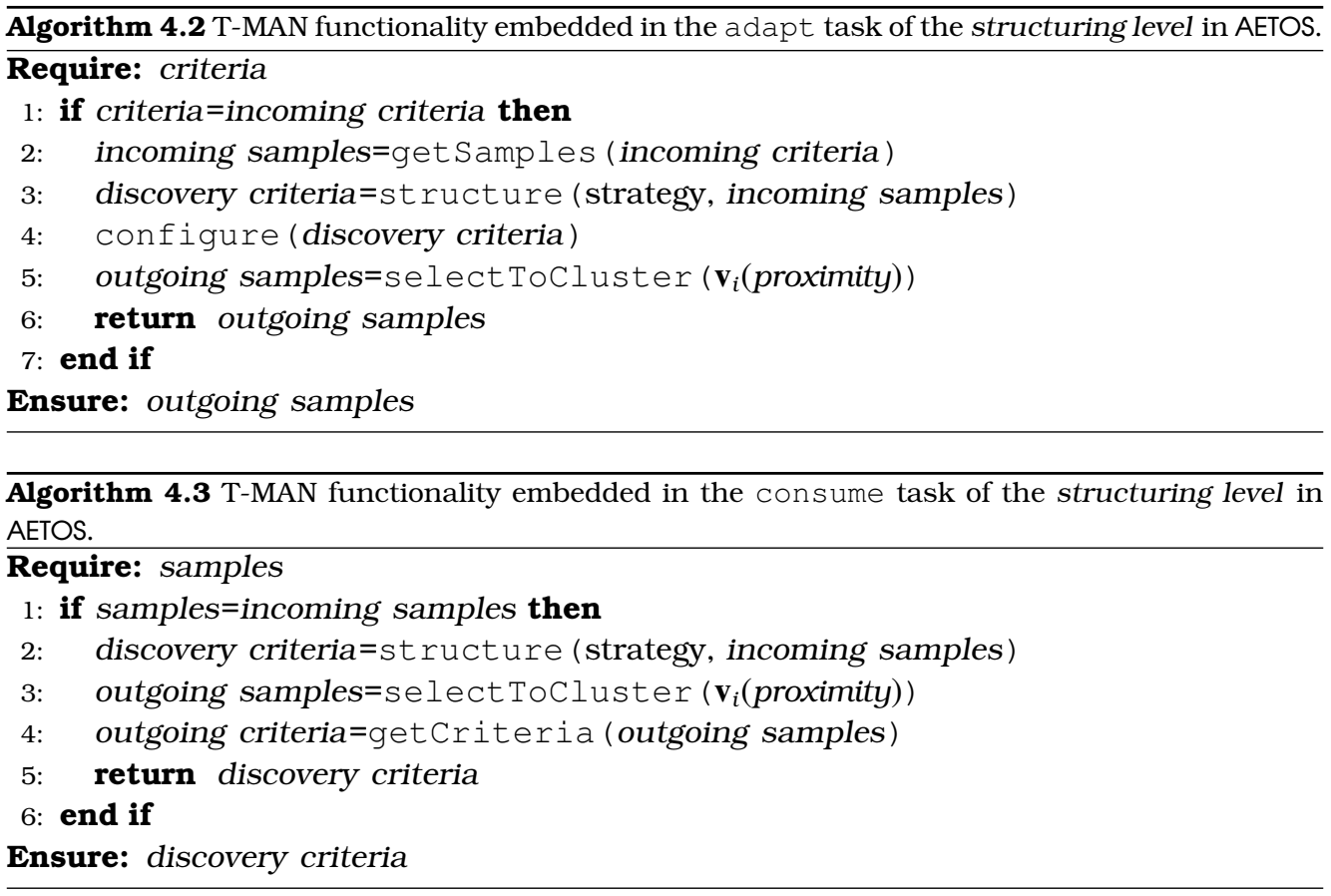

the outgoing samples respectively (line 5 and 3 of Algorithm 4.2 and 4.3 respectively). More in-depth technical details about the operations of T-MAN are provided by Jelasity et al. [2009]. The structure subtask (line 3 and 2 of Algorithm 4.2 and 4.3 respectively) performs the building of the proximity view according to the adopted adaptation strategy illustrated in Section 4.6.

\subsubsection{Adaptations}

The proximity view is not a simple static container of ranked nodes from which candidate parents and children are selected based on static criteria. In contrast with the random view and tree view, the proximity view is dynamic and its input is controlled by a number of adaptations illustrated in this section.

The agent of node $i$ includes in its proximity view nodes ranked in the range $\left[0, w_{i}\right) \cup$ $\left(w_{i}, 1\right)$ by default. Restricting this space results in a more targeted search for candidate parents and children given the limited size of the proximity view. Performing exclusions of a range of weights from the default range of weights $\left[0, w_{i}\right) \cup\left(w_{i}, 1\right)$ is the approach followed to limit this search space. Figure 4.6 illustrates the concept of such adaptations applied in the range of ranked nodes in proximity view. Four types of adaptations are applied:

- Reset: This adaptation defines the default range $\left[0, w_{i}\right) \cup\left(w_{i}, 1\right)$ of ranked nodes in the proximity view. It is applied if the proximity view remains empty for a period of time as a result of multiple upgrade and downgrade adaptations applied.

- Upgrade: This adaptation excludes nodes that are ranked with low weights in favor of the highly ranked nodes. The new range is defined as $\left(w_{c_{\vdash}}, w_{i}\right) \cup\left(w_{p_{\vdash}}, 1\right)$ with 


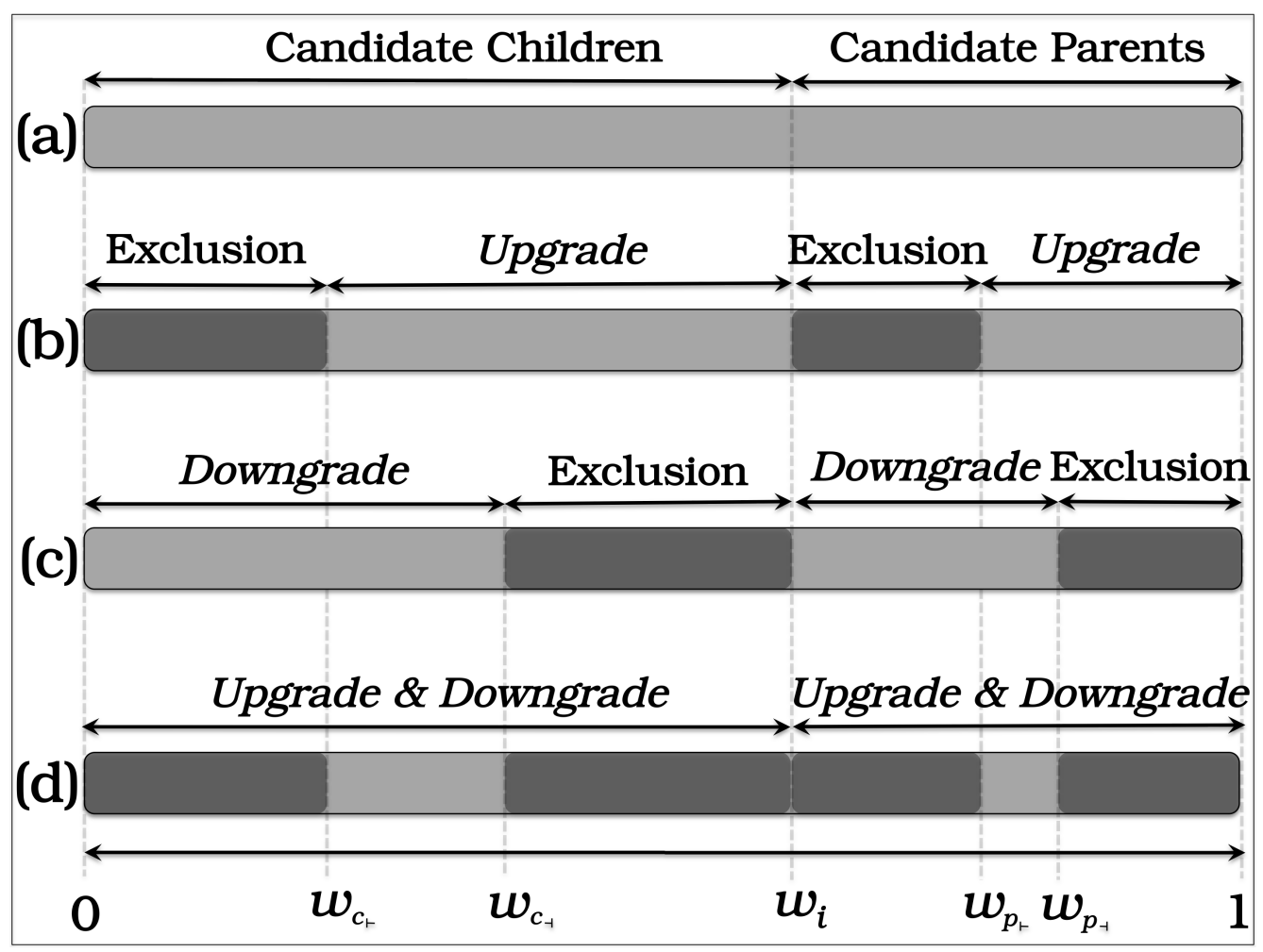

Figure 4.6: Adaptations in the range of ranked nodes in the proximity view: (a) reset adaptation, (b) upgrade adaptation, (c) downgrade adaptation, (d) upgrade and downgrade adaptation.

$c_{\vdash}$ and $p_{\vdash}$ indexing a child and parent respectively based on which this reconfiguration is defined. This adaptation results in potentially higher ranked candidate parents and children provided to the coordination level of AETOS.

- Downgrade: This adaptation excludes nodes that are ranked with high weights in favor of the low ranked nodes. The new range is defined as $\left[0, w_{c_{4}}\right) \cup\left(w_{i}, w_{p_{4}}\right)$ with $c_{\dashv}$ and $p_{\dashv}$ indexing a child and parent respectively based on which this reconfiguration is defined. This adaptation results in potentially lower ranked candidate parents and children provided to the coordination level of AETOS.

- Upgrade and downgrade: This adaptation excludes the low and highly ranked nodes and defines an in-between range from which candidate parents and children are selected. This new range is defined as $\left(w_{c_{\vdash}}, w_{c_{-}}\right) \cup\left(w_{p_{\vdash}}, w_{p_{-}}\right)$.

The purpose of adaptations is to improve the selections of candidate parents and children provided to the coordination level for building and maintaining the tree topology. The structuring criteria concerning the clustering of AETOS contain a positive or negative feedback about the earlier provided candidate parents and children. This feedback is associated with the applied adaptations. For example, positive feedback can be interpreted by the structuring level as selecting higher ranked candidate parents and children than before and therefore, positive feedback is associated with an upgrade 
adaptation. Note that a ranked node, such as the $w_{p_{\vdash}}, w_{p_{\uparrow}}, w_{c_{\vdash}}$ and $w_{c_{\uparrow}}$ in the above examples is a reference weight based on which adaptations are performed and is also part of the structuring criteria in AETOS.

Adaptations support more informed selections of candidate parents and children provided to the coordination level. The proximity view is constrained to higher or lower ranked candidate parents and children. The same approach can be applied for the actual selections provided to the coordination level: Highly or low ranked candidate parents and children may be preferred. Therefore adaptations and selections can be combined in various schemes forming adaptation strategies illustrated in Section 4.6.

\subsection{Building and Maintenance}

The coordination level is responsible for building and maintaining the tree topology. Self-organization is performed by coordinating parent-child links that respect the graph properties of nodes, e.g., degree-bounding. The links of the organized tree topologies should be bidirectional. For this reason, the agents of the coordination level contact the respective agents of the candidate parents/children provided by the structuring level to establish parent-child links that respect these properties. Links are established if and only if they are mutually accepted between communicating agents. Furthermore, a link can be removed by a connected node without mutual acceptance in favor of a new link with another node. Mutual acceptance of a link corresponds to positive feedback whereas rejection or removal of a link is negative feedback. This feedback is part of the structuring criteria provided to the structuring level.

Furthermore, the agents of the coordination level are competitive and seek to connect with the highest or lowest possible ranked parent and children. This requirement is part of the organizational criteria and therefore it is abstracted from an actual factor that influences the performance of an application. For simplicity, this chapter assumes that the highest ranked nodes are preferred.

AETOS introduces peer-to-peer coordination based on four types of exchanged messages:

- Request: This message is sent to a candidate parent (parent request) or candidate child (child request). It contains agent samples of the sender agent such as the weight of its node and its network identifier. A request is sent if and only if the tree view is not filled or the candidate parents and children provided by the structuring level are ranked higher than the parents and children in the tree view.

- Acknowledgment: This message is an acceptance of a request. Given a parent request, an acknowledgment is sent if the parent in the tree view is ranked lower than the sender node of the parent request. Similarly, given a child request, an acknowledgment is sent if the children in the tree view are not all ranked higher than the node of the sender node of the child request. The receipt of an acknowledgment follows the filling of the tree with a new parent or child and positive feedback to the structuring level. If these actions require the replacement of a parent or child from the tree view, a removal message is sent to this parent or child.

- Rejection: This message is denial of a request. For a parent request, a rejection is sent if the parent in the tree view is ranked higher than the sender node of the 
parent request. Similarly, in case of a child request, a rejection is sent if the children in the tree view are all ranked higher than the sender node of the child request. The receipt of a rejection follows a negative feedback to the structuring level.

- Removal: This message triggers deletion of a parent or child from the tree view. In case of a receipt of a removal message, negative feedback is provided to the structuring level.

Appendix A illustrates the communication algorithm of the agents in detail.

\subsection{Adaptation Strategies}

The structuring level of AETOS performs selection of ranked nodes within the adaptive range of the proximity view based on two concepts:

- Selecting candidate parents only, candidate children only, or both: three selection options

- Selecting the highest or lowest ranked candidate parents and children: two selection options

These selection options create $2^{3}=8$ combinations. These combinations are the adaptation strategies of AETOS and they are illustrated in Figure 4.7. Adaptation strategies provide (i) different preference schemes of candidate parents and children and (ii) different schemes for the applicability of adaptations in the range of ranked nodes in the proximity view as illustrated below:

- PRESBYOPIC: It defines the selection of the highest ranked candidate parents and the lowest ranked candidate children in the proximity view. A positive feedback is associated with an upgrade adaptation for the candidate parents and a downgrade adaptation for the candidate children of the proximity view. In contrast, a negative feedback corresponds to a downgrade adaptation for the candidate parents and an upgrade adaptation for the candidate children.

- MYOPIC: It defines the selection of the lowest ranked candidate parents and the highest ranked candidate children in the proximity view. A positive feedback is associated with a downgrade adaptation for the candidate parents and an upgrade adaptation for the candidate children of the proximity view. In contrast, a negative feedback corresponds to an upgrade adaptation for the candidate parents and a downgrade adaptation for the candidate children.

- HUMBLE: It defines the selection of the lowest ranked candidate parents and children in the proximity view. Positive feedback is associated with a downgrade adaptation for the candidate parents and children of the proximity view. In contrast, negative feedback corresponds to an upgrade adaptation for the candidate parents and children. 


\begin{tabular}{|c|c|c|c|c|}
\hline \multirow[b]{2}{*}{ Strategy } & \multicolumn{4}{|c|}{$\boldsymbol{w}_{i}{ }_{\text {Candidate Parents }}$} \\
\hline & Low & High & Low & High \\
\hline PRESBYOPIC & $\checkmark$ & & & $\nu$ \\
\hline MYOPIC & & $V$ & $\checkmark$ & \\
\hline HUMBLE & $\nu$ & & $V$ & \\
\hline GREEDY & & $V$ & & $\checkmark$ \\
\hline TOP-DOWN & & $\nu$ & & \\
\hline BOTTOM-UP & & & $\checkmark$ & \\
\hline TOP & & & & $\nu$ \\
\hline BOTTOM & $\checkmark$ & & & \\
\hline
\end{tabular}

Figure 4.7: The selection schemes of the adaptation strategies in AETOS.

- GREEDY: It defines the selection of the highest ranked candidate parents and children in the proximity view. Positive feedback is associated with an upgrade adaptation for the candidate parents and children of the proximity view. In contrast, negative feedback corresponds to a downgrade adaptation for the candidate parents and children.

- TOP-DOwn: It defines the selection of the highest ranked candidate children in the proximity view. Positive feedback is associated with an upgrade adaptation for the candidate children of the proximity view. In contrast, negative feedback corresponds to a downgrade adaptation for the candidate children.

- воттом-up: It defines the selection of the lowest ranked candidate parents in the proximity view. Positive feedback is associated with a downgrade adaptation for the candidate parents of the proximity view. In contrast, negative feedback corresponds to an upgrade adaptation for the candidate parents.

- TOP: It defines the selection of the highest ranked candidate parents in the proximity view. Positive feedback is associated with an upgrade adaptation for the candidate parents of the proximity view. In contrast, negative feedback corresponds to a downgrade adaptation for the candidate parents.

- воттом: It defines the selection of the lowest ranked candidate children in the 
proximity view. Positive feedback is associated with a downgrade adaptation for the candidate children of the proximity view. In contrast, negative feedback corresponds to an upgrade adaptation for the candidate children.

Note that the adaptation strategies are not aware of the actual graph properties of an organized tree topology. For example, an adaptation strategy does not contain any information about the degree-bounding or the completeness of the tree topology, properties that are all handled in the coordination level. The adaptation strategies are a number of generic, highly modular and customizable selection schemes for coupling nodes. The actual utilization and adoption of these strategies define the resulting topology that emerges from this coupling of nodes based on their proximity. In AETOS, the utilization of an adaptation strategy is controlled by the feedback contained in the structuring criteria. This feedback defines the applied adaptation in the proximity view. The adoption of an adaptation strategy is performed as follows:

- Static adoption: An adaptation strategy is adopted as a system parameter. In this case, an adaptation strategy is designed explicitly to serve the self-organization of one or more specific topologies.

- Dynamic adoption: The adopted adaptation strategy changes dynamically during system runtime. However, the decision for change is based on static criteria selected empirically or based on some monitored parameters. For example, the adopted adaptation strategy may change after some runtime period or after a topology change, e.g., insertion of new nodes in the network.

- Emergent adoption: The adopted adaptation strategy changes dynamically during system runtime based on the feedback provided by the coordination level. This approach requires the definition and reasoning of transitions from the one adopted adaptation strategy to another. For example, if the selection of candidate parents and children based on the GREEDY adaptation strategy results in a negative feedback, then a possible transition is the adoption of the HUMBLE strategy.

Note that the adopt subtask of Algorithm 3.9 realizes the adoption of an adaptation strategy. Moreover, the select ToProvide subtask of the same algorithm adapts the range of ranked nodes in the proximity view from which the candidate parents and children are selected as defined by the adopted strategy. The structure subtask of Algorithm 3.10 inserts in the proximity view the ranked nodes contained in the discovered samples upon their weights belong in the range defined by the adopted strategy. Finally, the eight adaptation strategies of AETOS are not the only strategies that can be designed. Additional selection options of candidate parents and children can be defined resulting in new adaptation strategies. The purpose of this chapter is to introduce the concept of an adaptation strategy instead of illustrating an exhaustive applicability of this concept.

\subsection{Experimental Evaluation}

AETOS is implemented and evaluated in Protopeer [Galuba et al., 2009], a prototyping toolkit for distributed systems illustrated in Section 2.4. A concurrent implementation of AETOS in the AgentScape simulation framework [Oey et al., 2010a] confirms the 
results of Protopeer. Table 4.1 summarizes the selected experimental settings. Note that multiple values for a single parameter denote the tested variations of this parameter in some of the illustrated experiments. The values depicted with bold are the default ones.

Table 4.1: The experimental settings for the evaluation of AETOS. The bold values are the default ones in the performed experiments.

\begin{tabular}{|c|c|c|}
\hline & Parameter & Value \\
\hline \multirow{6}{*}{ AETOS } & $n$ & 1500 \\
\hline & $t(\mathrm{AETOS})$ & 400 \\
\hline & $T$ (AETOS) & 1000 \\
\hline & $t^{\prime}(\mathrm{AETOS})$ & 6 \\
\hline & $w \in[0,1)$ & random \\
\hline & $\mid \mathbf{v}($ ring $) \mid$ & 5 \\
\hline \multirow{3}{*}{ Coordination level } & $k$ & $3, \mathbf{4}, 6$, varied \\
\hline & $z$ & 2 \\
\hline & $T$ (coordination level) & 1000 \\
\hline \multirow{9}{*}{ Structuring level } & $q$ & 30 \\
\hline & $f$ & 6 \\
\hline & strategy adoption & static, dynamic \\
\hline & $T($ reset $)$ & $7, \mathbf{1 5}, 30$ \\
\hline & strategy adoption & static, dynamic \\
\hline & $T$ (reset) & $2000, \mathbf{3 0 0 0}, 5000, \infty$ \\
\hline & T-MAN inclusion & enabled, disabled \\
\hline & $t^{\prime}$ (structuring level) & 6 \\
\hline & $T$ (structuring level) & $100, \mathbf{2 0 0}, 250,500,1000$ \\
\hline \multirow{5}{*}{ Discovery level } & $r$ & 50 \\
\hline & view selection policy & swapper \\
\hline & view propagation policy & push-pull \\
\hline & peer selection policy & random \\
\hline & $T$ (discovery level) & $100, \mathbf{2 0 0}, 250,500,1000$ \\
\hline
\end{tabular}

A network of 1500 peers is simulated running AETOS for $t$ (AETOS) $=400$ epochs. Every epoch lasts for $T($ AETOS $)=1000 \mathrm{~ms}$. Protopeer initially bootstraps peers in a ring topology. The bootstrapping time period is $t^{\prime}$ (AETOS) $=6$ epochs and the size of the ring view $\mid \mathbf{v}($ ring $) \mid=5$ for every peer. Every node of the tree topology is ranked with a random weight $w \in[0,1)$.

The coordination level defines the number of children $k=4$. Other topologies with $k=3,5$ and a random number $k$ of 3,4 or 5 children for each node of the tree topology are evaluated. Furthermore, the coordination level sends $z=2$ number of request messages. If the adopted adaptation strategy includes both candidate parents and children in the proximity view, the requests are one for each candidate type. Finally the request messages are sent periodically every $T$ (coordination level $)=1000 \mathrm{~ms}$.

In the structuring level, the size of the proximity view is $q=30$ with the scaling factor $^{5}$ chosen to be $f=6$. The static and dynamic adoption schemes of adaptation strategies are evaluated. Adaptations and the T-MAN mechanism are enabled and

\footnotetext{
${ }^{5}$ In the case that the number of children for each node is $k=4$, the proximity view contains 6 candidate parents and 24 candidate children as defined by $q_{i}=f *\left(k_{i}+1\right)$.
} 
disabled in some of the experiments. When adaptations are enabled, the reseting period is chosen to be $T$ (reset) $=3000 \mathrm{~ms}$. The alternative values of 2000,5000 , and the case of no reseting are examined. Moreover, when T-MAN is enabled, it runs with the parameters $m=40, \psi=20, l=6$. The role of these parameters is out of the scope of this thesis and is illustrated by Jelasity et al. [2009]. Finally, the structuring level is bootstrapped within $t^{\prime}$ (structuring level) $=6$ epochs and its execution period is selected at $T$ (structuring level $)=200 \mathrm{~ms}$. The alternative values of 100, 250, 500 and $1000 \mathrm{~ms}$ are also examined.

The discovery level is realized by the peer sampling service [Jelasity et al., 2007]. The size of the random view is $r=50$ and the execution period is $T$ (discovery level) $=$ $T($ AETOS) $/ 5=200 \mathrm{~ms}$. Other values examined are 100, 250, 500 and $1000 \mathrm{~ms}$. The values of the 'view selection', 'view propagation' and 'peer selection' policies are selected to maximize the randomness and dissemination speed of gossiping. More information about these parameters are provided by Jelasity et al. [2007].

\subsubsection{Evaluation outline}

The evaluation of AETOS is based on the four performance metrics illustrated in Section 4.1: connectedness $\beta$, connectivity $\gamma$, fitness $\rho$ and instability $\delta()$. These four metrics represent the quality of the AETOS overlay service. The optimum fitness of a tree for given graph properties is computed in a centralized fashion as illustrated in Algorithm 4.1. Note that the experiments performed specifically focus on the convergence of connectedness, connectivity and fitness during system runtime of $t$ (AETOS) $=400$ epochs. Furthermore, the instability of a performance metric is computed after the system has converged to its average maximum value. The number of messages $\lambda($ level) generated by each level of AETOS is also computed as a measure of the communication cost. Finally, qualitative comparisons are performed by visualizing the self-organized tree topologies using the $\mathrm{JUNG}^{6}$ visualization library of O'Madadhain et al. [2005]. The trees are visualized in a radial layout that depicts clearer the balancing and completeness graph properties. ${ }^{7}$

Six aspects of AETOS are examined in the performed experiments:

1. The adaptation strategies.

2. The effect of adaptations.

3. The dynamic adoption of the adaptation strategies.

4. The periodical reset of the adaptations.

5. Tree topologies with different graph properties.

6. The adjustment of periodical executions in the three levels of AETOS.

7. The effect of the T-MAN mechanism.

The rest of this section illustrates the experimental results for each of these aspects.

\footnotetext{
${ }^{6}$ Available at http: / / jung. sourceforge. net (Last accessed: May 2012)

${ }^{7}$ In the colored version on this thesis, the trees are visualized with blue and red nodes. The blue nodes indicate the ones that form the main tree. In contrast, the red nodes represent the ones that are disconnected from the main body of the tree.
} 


\subsubsection{Adaptation strategies}

Figure 4.8 illustrates the convergence of connectedness for the eight adaptation strategies. For higher readability, the figures of this section that concern the adaptation strategies are split in four subgraphs, (a)-(d), each showing the results of two adaptation strategies. The order represents the performance of the adaptation strategies, starting from the adaptation strategy with the highest performance to the adaptation strategy with the lowest one. The MYOPIC, HUMBLE, BOTTOM-UP and TOP-DOwn adaptation strategies converge to the maximum connectedness of 1 within the first 40 epochs as depicted in Figure $4.8 \mathrm{a}$ and $4.8 \mathrm{~b}$. The instability of connectedness in MYOPIC and HUMBLE is approximately 0.002 . For BOTTOM-UP and TOP-DOWN, instability increases to 0.004 and 0.007 respectively. In contrast, the GREEDY, PRESBYOPIC, TOP and BOTTOM adaptation strategies follow in Figure $4.8 \mathrm{c}$ and $4.8 \mathrm{~d}$ at a lower performance. More specifically, GREEDY and PRESBYOPIC converge approximately 20 epochs slower and reach an average connectedness of 0.82 . Their instability is 0.029 and 0.026 respectively. Top is even more inefficient with an average connectedness of 0.54 and an instability of 0.067 . The воттом strategy does not converge and results in multiple disconnected parent-child links.

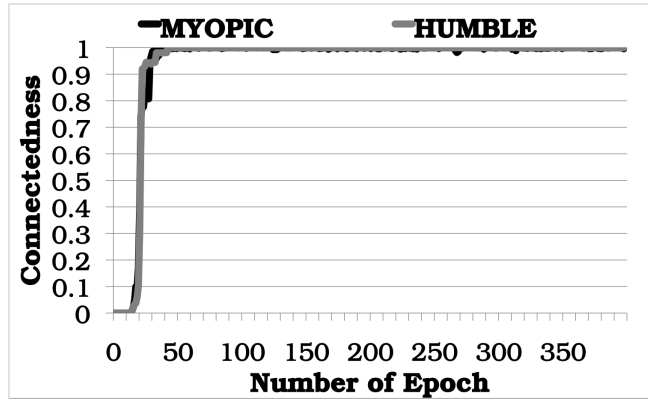

(a) MYopIC and HuMBLE.

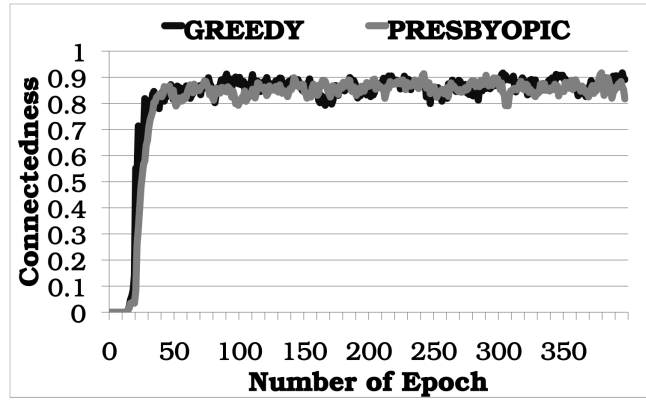

(c) GREEDY and PRESBYOPIC.

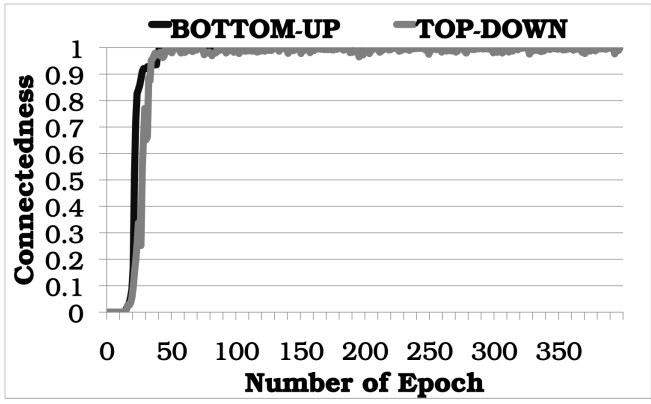

(b) Воттом-Up and Top-Down.

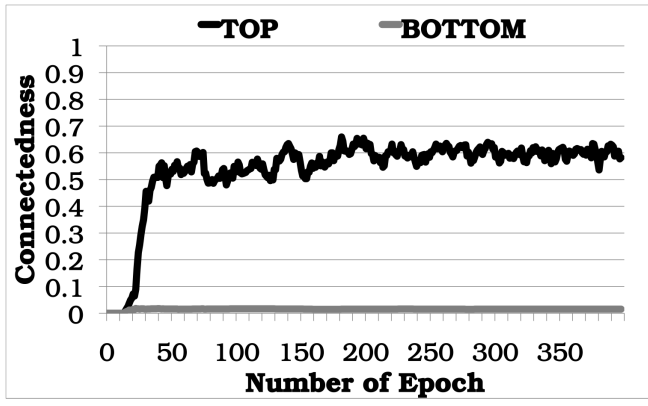

(d) Top and воттом.

Figure 4.8: Convergence of connectedness for the adaptation strategies.

Figure 4.9 illustrates the convergence of connectivity for the eight adaptation strategies. The performance comparisons of this performance metric are similar with the ones of connectedness. The MYOPIC, HUMBLE, вотTOM-UP and TOP-DOwn of Figure 4.9a and $4.9 \mathrm{~b}$ converge to the maximum connectivity of 1 in fewer than 30 epochs. The 
instability of connectivity in these strategies is approximately 0 . In contrast, PRESBYOPIC and GREEDY in Figure 4.9c converge to an average maximum connectivity of 0.95 in approximately 40 epochs with an instability of 0.012 . Despite the relative low connectivity of the воттом adaptation strategy shown in Figure 4.9d, it converges to an average maximum connectivity of 0.85 and an instability of 0 in approximately 40 epochs. This is because there is a significant number of parent-child links formed whose nodes remain disconnected between each other. In other words, these links remain independent and decoupled. Finally, TOP, also depicted in Figure 4.9d, achieves the lowest connectivity. The average maximum connectivity is approximately 0.67 with an instability of 0.031 .

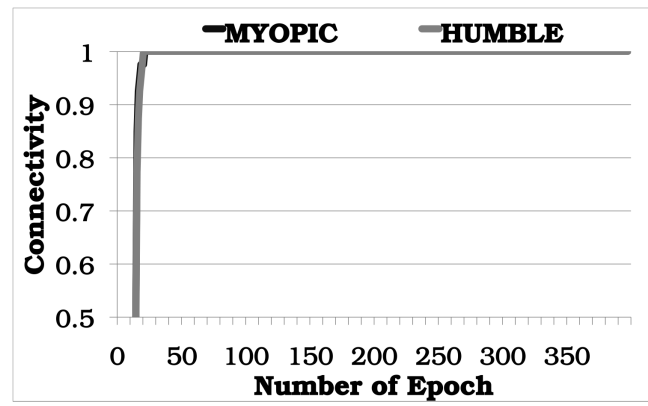

(a) MYopic and HUMBLE.

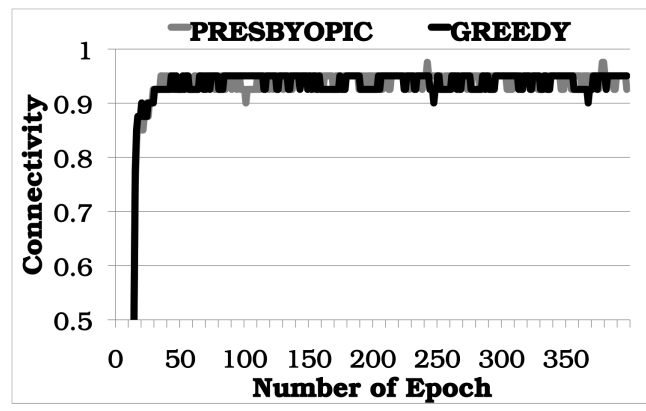

(c) Presbyopic and greedy.

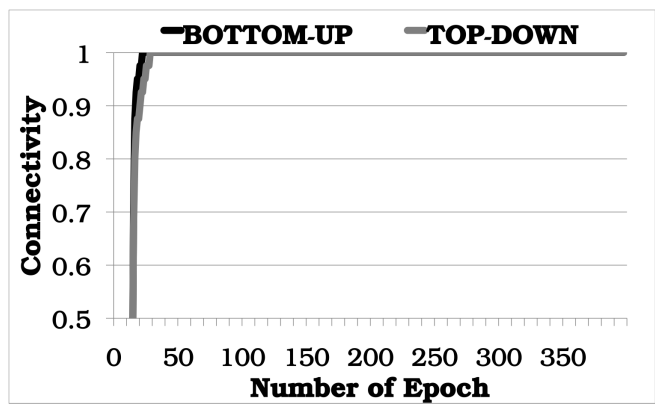

(b) BотTOM-UP and TOP-DOwN.

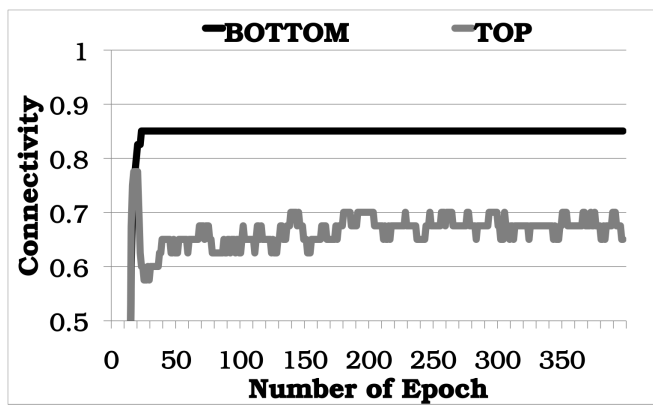

(d) Bоттом and Top.

Figure 4.9: Convergence of connectivity for the adaptation strategies.

Note that the MYOPIC, HUMBLE, BOTTOM-UP and TOP-DOWN adaptation strategies that achieve the highest connectedness and connectivity do not define highly ranked candidate parents in the proximity view of agents. Furthermore, воттом is ineffective as selection of the lowest candidate children is not consistent with the selection criteria of the coordination level that defined a preference for the highest possible parents and children.

Figure 4.10 illustrates the fitness of the tree topology built by each adaptation strategy. The performance comparisons show different results in this case. Top-Down and MYOPIC converge to the average maximum fitness of 0.95 in more than 150 epochs as depicted in Figure 4.10a. The instability of their fitness is 0.007 and 0.008 respectively. Therefore, these two strategies provide the highest fitness and the slowest convergence, yet the fitness is higher than the fitness in the other adaptation strategies during this convergence period. Humble and воттом, illustrated in Figure 4.10b, follow with an 
average maximum fitness of 0.87 and 0.86 respectively and instability of 0 . These two adaptation strategies converge in less time, approaching their maximum fitness in approximately 60 epochs. Figure $4.10 \mathrm{c}$ shows the results of PRESBYOPIC and GREEDY. The average maximum fitness is 0.85 and 0.83 respectively, converging in approximately 40 epochs. Their instability is 0.011 and 0.018 respectively. Finally, the тоР and воттомUP adaptation strategies of Figure 4.10d result in the lowest fitness of 0.72 and 0.65 respectively. Top converges in more than 100 epochs, whereas the воттом-UP converges in 30 epochs. In contrast to the воттом-UP that has an instability of 0 , the тоP has the highest instability of 0.025 .

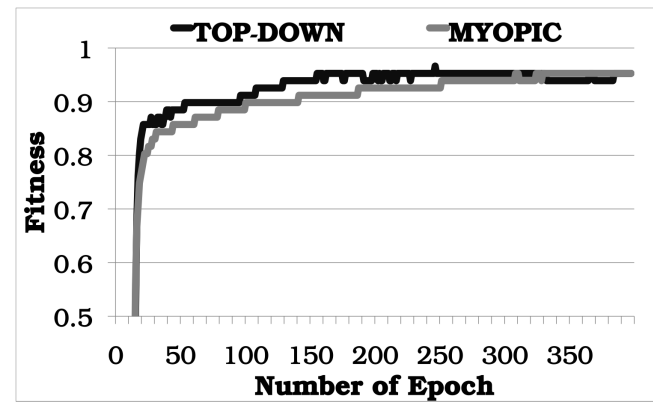

(a) TOP-DOwn and MYOPIC.

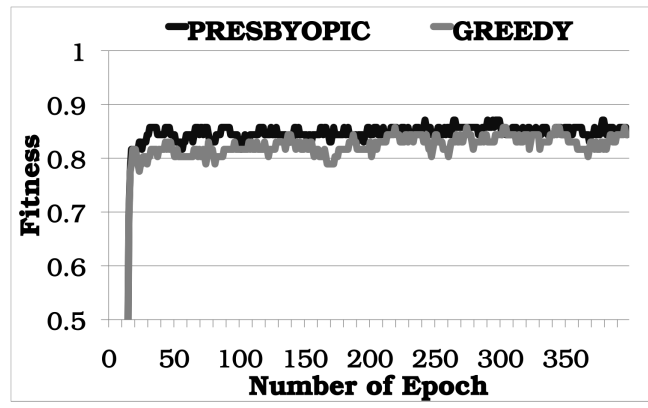

(c) PResbyopic and Greedy.

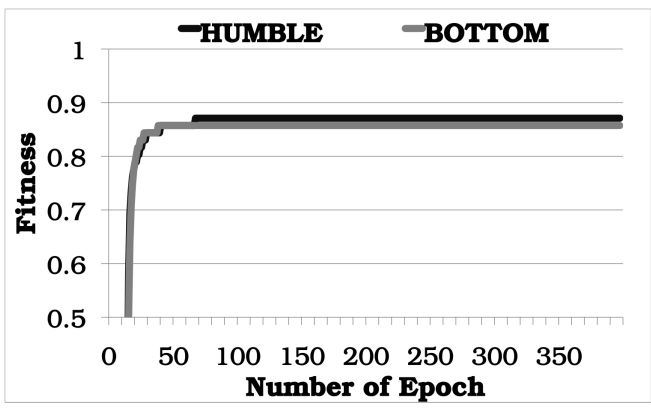

(b) Humble and воттом.

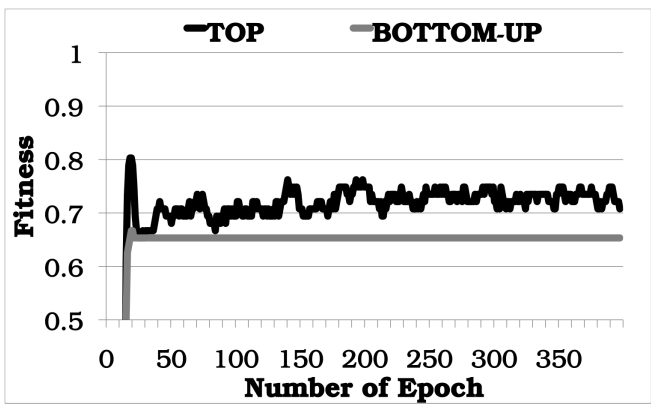

(d) Top and воттом-UP.

Figure 4.10: Convergence of fitness for the adaptation strategies.

Note that the воттом adaptation strategy achieves a relatively high fitness but low connectedness and connectivity. This is because the highly ranked nodes are preferred in the coordination level and therefore they connect with the lowest ranked nodes resulting in robust parent-child links.

The communication cost of the discovery level and the structuring level is constant and depends on the gossiping period of the peer sampling service and T-MAN respectively. During the runtime of these 2 gossiping protocols, agents exchange periodically 2 messages in each case as both protocols are 'push-pull'. Furthermore, each gossiping protocol is executed 5 times during an epoch as $T$ (discovery level) $=$ $T($ structuring level $)=T($ AETOS $) / 5=1000 / 5=200 \mathrm{~ms}$. Therefore, the total number of messages that an agent of the discovery level and the structuring level sends during an epoch is $2 * 2 * 5=20$ messages or $\lambda($ discovery level $)+\lambda($ structuring level $)=20 * 1500=$ 30000 messages for the total number of these agents in the network. Results confirm 
these estimations.

In contrast, the communication cost of the coordination level is influenced by the adopted strategy. Figure 4.11 illustrates the convergence of this communication cost. All adaptation strategies converge in fewer than 40 epochs. In Figure $4.11 \mathrm{a}$, the воттомUP adaptation strategy minimizes the number of exchanged messages to 0 in 30 epochs. At the same figure, the communication cost of the HUMBLE adaptation strategy consumes 2100 messages per epoch in average after its convergence. The instability of the communication cost is approximately 0.019. Mүоріс and воттом of Figure $4.11 \mathrm{~b}$ converge to an average maximum communication cost of 2600 and 4600 messages respectively. The instability of the communication cost is 0.017 and 0.015 respectively. In Figure $4.11 \mathrm{c}$, PRESBYOPIC and TOP-DOWN have a communication cost of 5300 and 5400 messages per epoch with an instability of 0.018. Finally, GREEDY and TOP have the highest average maximum communication cost of 5700 and 6300 messages and an instability of 0.017 and 0.02 respectively.

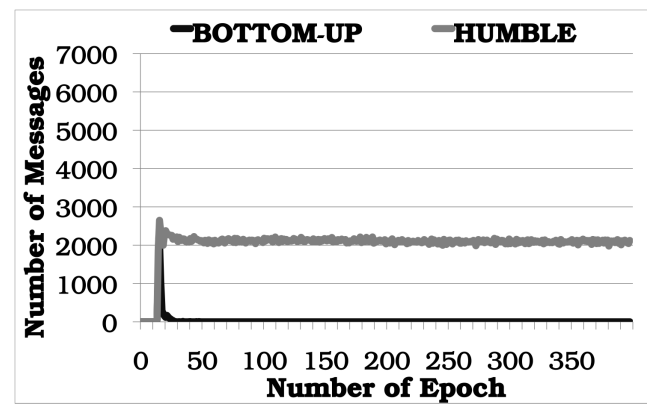

(a) BотTом-UP and HUMBLE.

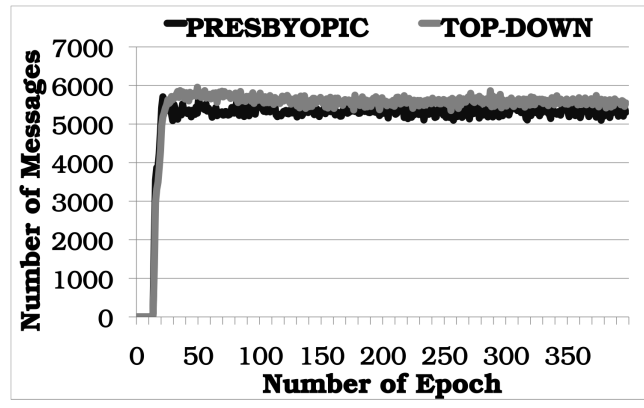

(c) PREsbyopic and TOP-DOwn.

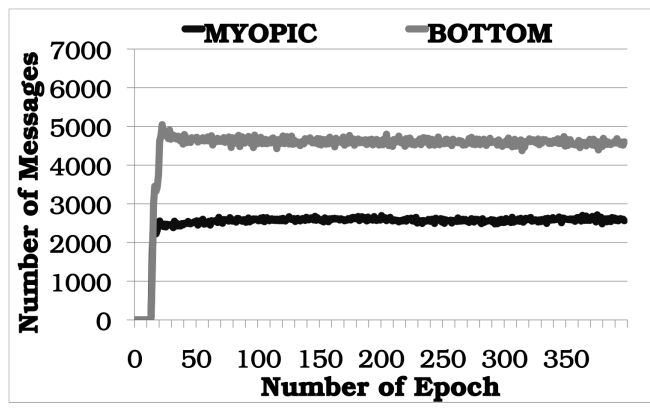

(b) Myopic and воттом.

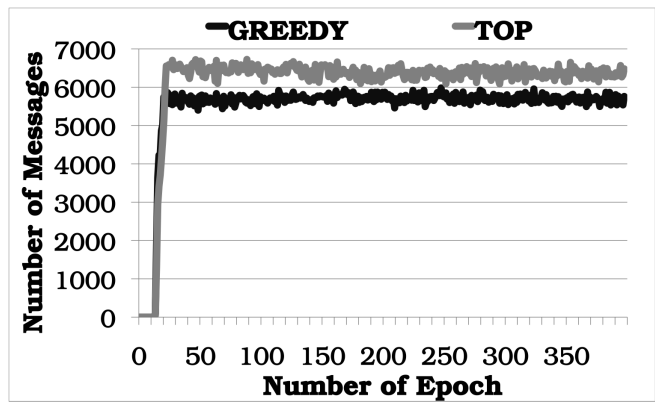

(d) GReEdy and Top.

Figure 4.11: Convergence of messages exchanged at the coordination level for the adaptation strategies.

Figure 4.12 shows the optimally organized topology computed in a centralized fashion as shown in Algorithm 4.1. Table 4.2 provides a visualization of the eight adaptation strategies at four time points during convergence: $20^{\text {th }}, 35^{\text {th }}, 50^{\text {th }}$ and $350^{\text {th }}$ epoch.

Visually, the GREEDY and PRESBYOPIC adaptation strategies result in similar topologies: Well balanced branches close to the root that become less balanced and less complete close to the leaves of the tree. A few disconnected branches are present. The Top adaptation strategy has the effect of a large well balanced and connected branch disconnected 


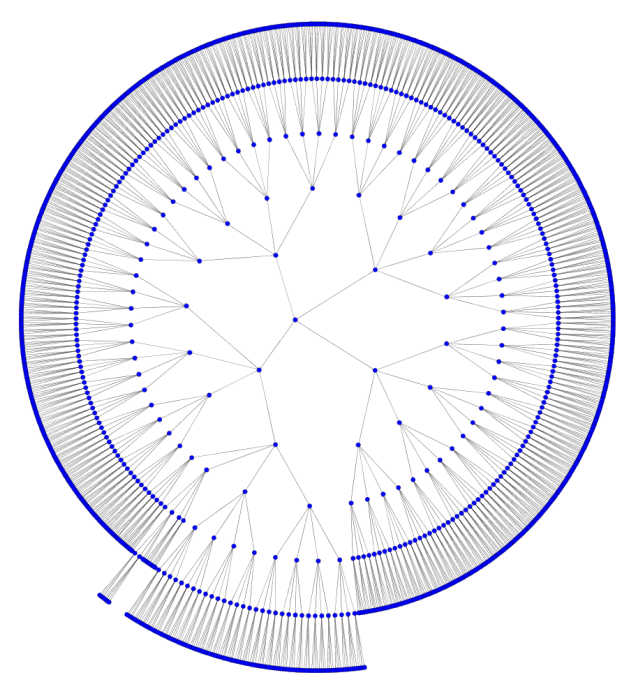

Figure 4.12: Visualization of the optimally organized tree topology of 1500 nodes.

from a large number of smaller branches. MYOPIC, HUMBLE, and вотTOM-UP result in significantly better connected topologies that have, however, imbalanced branches. From these three adaptation strategies, the imbalance of HUMBLE is the highest. Moreover, the topology of TOP-DOWN is organizationally the closest to the optimally organized tree topology of Figure 4.12. Most branches are balanced and there is a minimum number of disconnected nodes. Finally, the воттом adaptation strategy results in a forest with a large number of disconnected trees without a distinction of the main tree (branch).

\subsubsection{The effect of adaptations}

The reset, upgrade and downgrade adaptations of the proximity view contribute significantly in the achieved performance of the adaptation strategies. Without adaptations, meaning only performing selections and ignoring the feedback provided by the coordination level to the structuring level, the connectedness, connectivity, and fitness remain low with increased instability. However, communication cost decreases without adaptations as fewer connections are negotiated in the coordination level.

For example, the achieved connectedness without adaptations is approximately $20 \%$ lower for GREEDY and 10\% lower for MYOPIC compared with the case in which adaptations are enabled in the system. Fitness is almost double for GREEDY and 20\% higher for MYOPIC when using adaptations. In contrast, the communication cost with adaptations increases $30 \%$ for GREEDY and more than double for MYOPIC.

\subsubsection{Dynamic adoption of the adaptation strategies}

Section 4.7.2 shows that adaptation strategies result in performance trade-offs. Motivated by this observation, this section shows how adaptation strategies can be combined to collectively achieve higher performance than each one individually. This section describes a number of possibilities rather than an exhaustive illustration of how adap- 
Table 4.2: Visualization of the adaptation strategies at four time points during the runtime of AETOS: (i) $20^{\text {th }}$, (ii) $35^{\text {th }}$, (iii) $50^{\text {th }}$ and (iv) $350^{\text {th }}$ epoch.

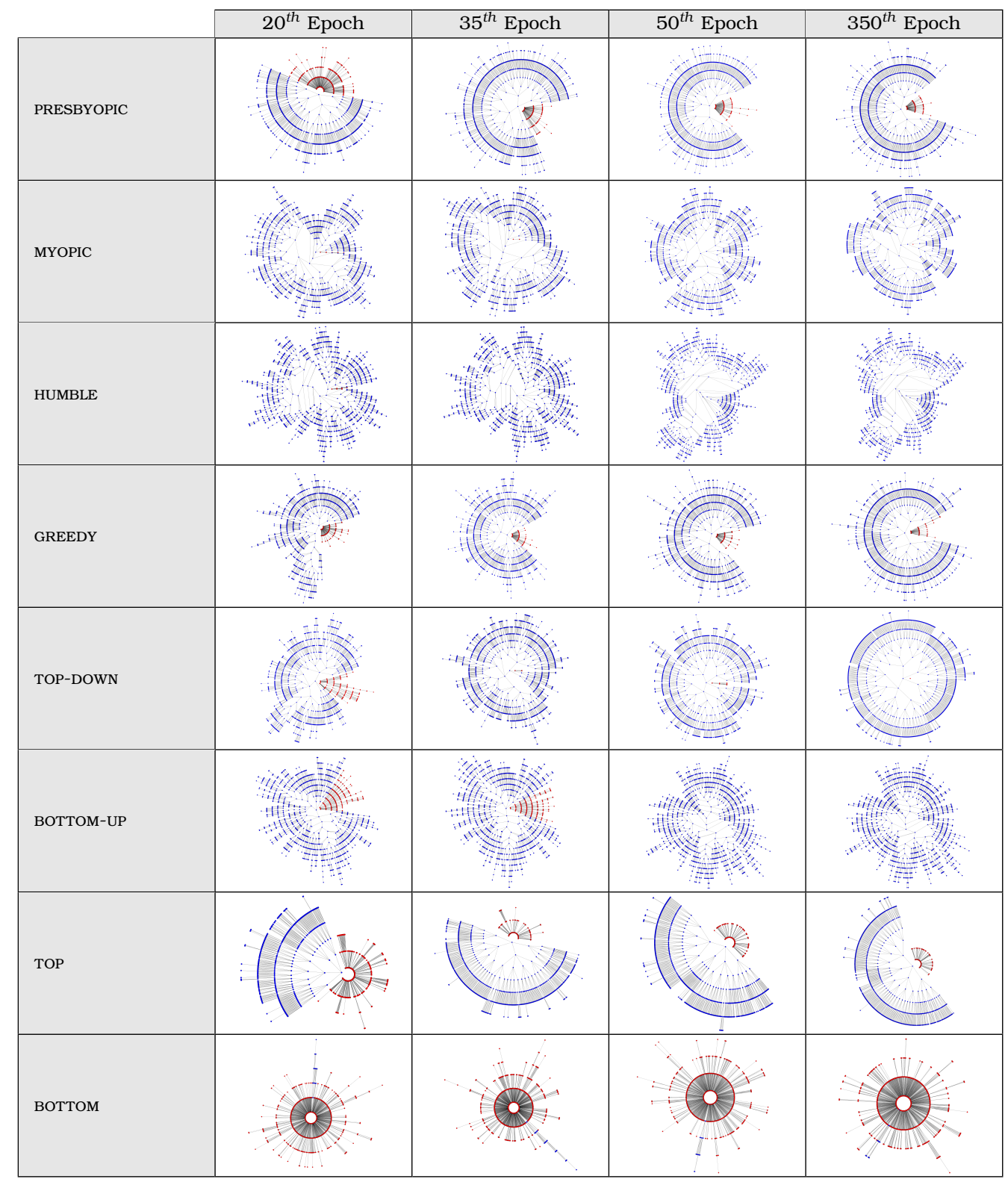

tation strategies can be adopted dynamically. Self-organization of a tree based on the synergy of two or more adaptation strategies is referred to as hybrid adaptation strategy.

Hybrid adaptation strategies introduced in this section are based on the following intuition. Certain adaptation strategies, such as TOP, GREEDY and PRESBYOPIC cannot meet the requirement of building a fully connected tree. They also consume a high 
number of messages without approaching the fitness of TOP-DOWN or MYOPIC. In contrast, the воттом-UP builds a fully connected tree at a minimum communication cost but with significantly lower fitness than the aforementioned adaptation strategies. Therefore, the possibility of combining the воттом-UP with one of the other adaptation strategies potentially results in a maximization of connectedness, fitness and a minimization of the communication cost. This section experimentally investigates this intuition.

Three hybrid adaptation strategies are examined: (i) TOP $\rightarrow$ BOTTOM-UP $\Rightarrow$ HYBRID-01, (ii) GREEDY $\rightarrow$ BOTTOM-UP $\Rightarrow$ HYBRID-02, and (iii) PRESBYOPIC $\rightarrow$ BOTTOM-UP $\Rightarrow$ HYBRID-03. AETOS is initiated with the former adaptation strategy in each case. After the 250th epoch, the nodes that do not have a parent, therefore they are disconnected from the main body of the tree, switch $(\leftrightarrow)$ to the воттом-Uр adaptation strategy.

Figure 4.13 illustrates connectedness, connectivity, fitness and communication cost of the three hybrid adaptation strategies. Note that all hybrid adaptation strategies inherit the property of a maximum connectedness and connectivity from the воттомUP. After the 250th epoch, both metrics converge within a short time to the maximum of 1.0 as depicted in Figure $4.13 \mathrm{a}$ and $4.13 \mathrm{~b}$. Note also the significant reduction of the communication cost in Figure 4.13d. HYBRID-01 minimizes this cost to 0, whereas, HYBRID-02 and HYBRID-03 continue generating approximately 700 and 1800 messages per epoch respectively. Fitness has an increase of 10\% and 8\% for HYBRID-02 and HYBRID-03 respectively as shown in Figure 4.13c. However, this is not the case for HYBRID-01 in which adoption of воттом-UP negatively influences the fitness by approximately $10 \%$.

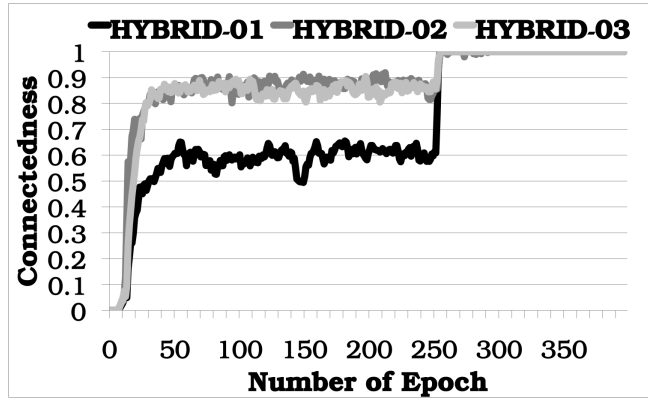

(a) Connectedness.

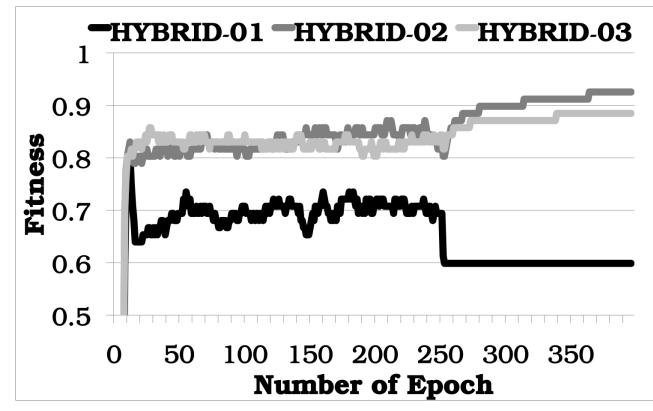

(c) Fitness.

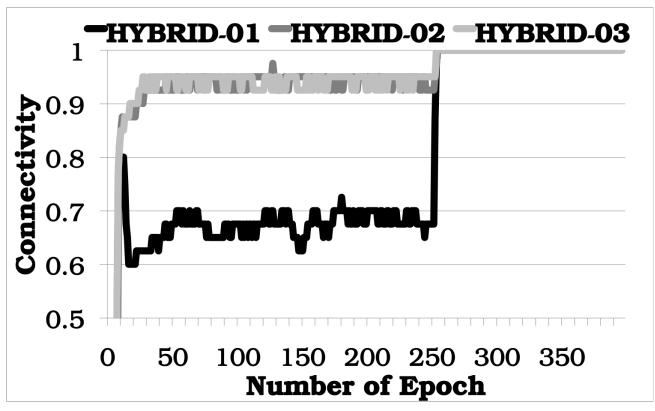

(b) Connectivity.

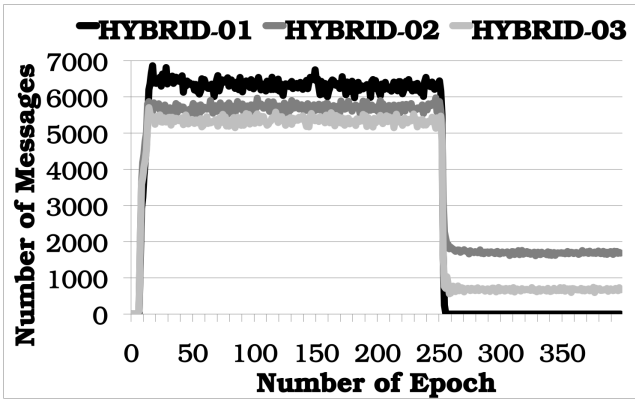

(d) Number of messages generated by the coordination level of AETOS.

Figure 4.13: The performance of the hybrid adaptation strategies. 
Table 4.3 visualizes the three hybrid strategies at three different points during runtime: (i) On the $245^{\text {th }}$ epoch, that is 5 epochs before воттом-UP is adopted, (ii) on the $255^{\text {th }}$ epoch, that is 5 epochs after the dynamic adoption of воттом-UP and (iii) on the $380^{\text {th }}$ epoch. This table shows clearly transitions of the adaptation strategy and their effect. The disconnected branches are connected to the leaves of the main tree forming a fully connected tree. The deterioration of fitness in HYBRID-01 is explained by the long 'lists' of nodes attached at the bottom of the tree. This attachment is more uniform for HYBRID-02 and HYBRID-03.

Table 4.3: Visualization of the tree topologies at three different epochs for three hybrid strategies on the (i) $245^{\text {th }}$, (ii) $255^{\text {th }}$ and (iii) $380^{\text {th }}$ epoch.

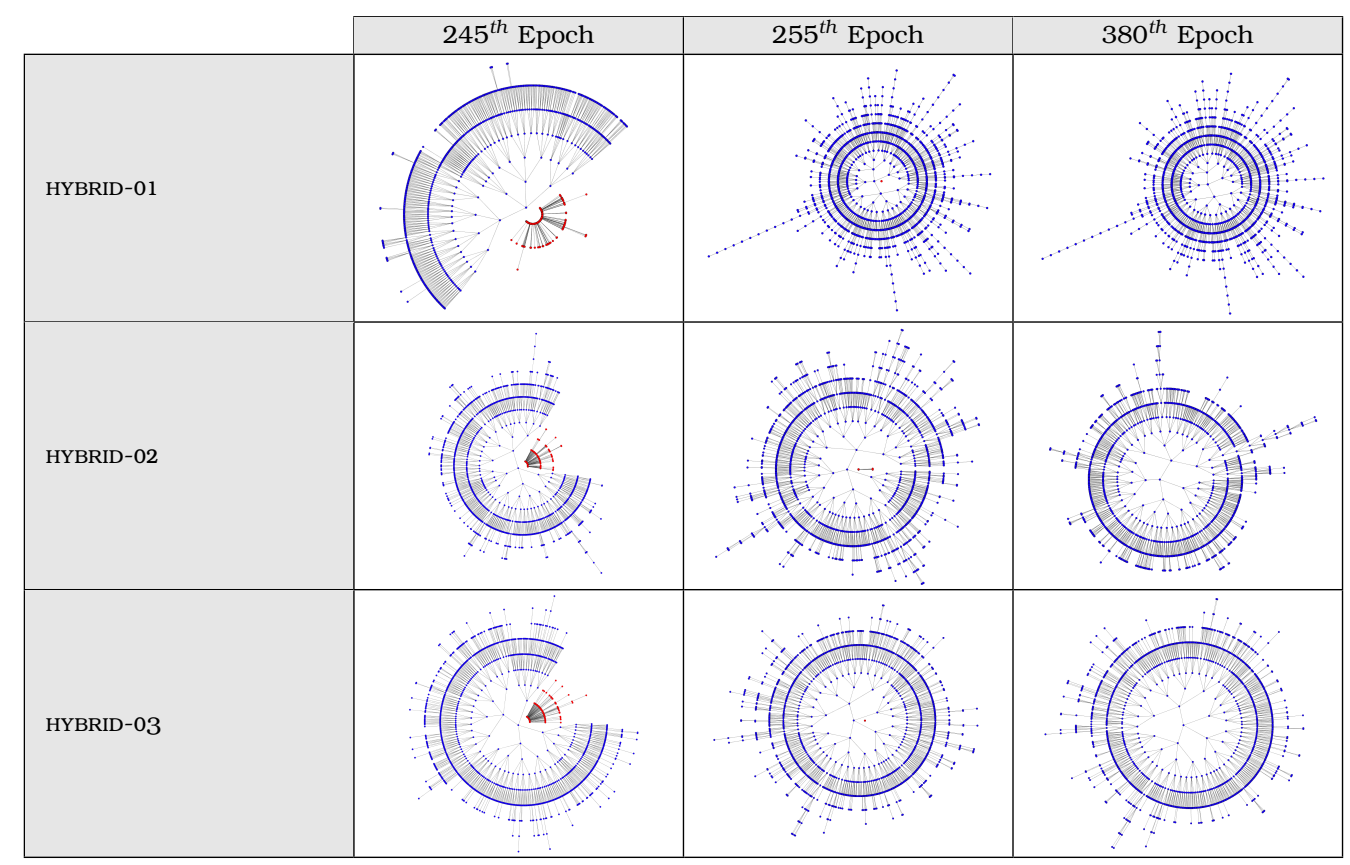

Note that hybrid adaptation strategies achieve a highly comparable performance to TOP-DOWN and MYOPIC. Furthermore, they suggest additional performance trade-offs. For example, the HYBRID-02 and HYBRID-03 achieve a significantly lower communication cost at a price of $3 \%-5 \%$ lower fitness compared to TOP-DOwn and MYOPIC.

\subsubsection{Reseting adaptations}

The reset adaptation is applied to the proximity view if it remains empty for a period of time. This is because multiple upgrade and downgrade adaptations applied may restrict the range of ranked candidate parents and children to such an extent that excludes all ranked nodes. This may be the case at the beginning of self-organization in which temporary links that are removed later on affect the proximity view of other nodes via the applied adaptations. In this case, the system is actually 'trapped' in a few locally optimum parent-child links, leaving a large number of nodes isolated with an 
empty proximity view. Conditional and periodic reset adaptation solves this problem.

Experimental evaluation confirms this intuition. Without reset adaptations, connectedness achieved with GREEDY remains lower than 0.45 for more than 160 epochs. Fitness remains high as few links formed are highly robust with nodes close to the root getting connected with the leaves of the tree in the respective optimum topology. However, the difference is insignificant to claim an improved fitness in the case of no adaptations in the proximity view. When $T$ (reset) decreases to 5, 3 and 2 epochs, the performance metrics converge fast as shown in Section 4.7.2.

Note that the experimental results also show that the lowest reseting period of $T($ reset $)=2$ epochs results in the highest communication cost as the probability of sending parent and child requests to the same nodes increases when adaptations are reset more frequently. For GREEDY, the communication cost is $2 \%$ higher for $T$ (reset) $=2$ compared with $T($ reset $)=5$.

\subsubsection{Tree topologies with different graph properties}

Three different tree topologies are evaluated: (i) A 3-ary tree, (ii) a 5-ary tree and (iii) a tree with randomly selecting 3,4 or 5 children per node. These tree topologies represent different distribution of links among their nodes. An application that uses a 5-ary tree may require higher computational and network resources in peers than a 3-ary tree, e.g., bandwidth [Tan et al., 2005a]. Results show a clear trend for most of the adaptation strategies. More levels result in a higher complexity meaning that the performance of AETOS degrades as the size of the tree view decreases. Therefore, a 3-ary tree performs worse compared to a 5-ary tree that has a fewer number of levels.

Table 4.4 illustrates performance results of the eight adaptation strategies for each tree topology. Fitness, connectedness and connectivity of a 3-ary tree topology is lower and their convergence slower for the PRESBYOPIC, MYOPIC, HUMBLE, GREEDY, TOP-DOWN, and TOP adaptation strategies. However, the communication cost is higher for the 5-ary tree in these adaptation strategies. For example the fitness for GREEDY is $\rho=0.77$ on the 380th epoch for a 3-ary tree whereas for the 5-ary tree is $\rho=0.87$. The respective values for the rest of the adaptation strategies are $\rho=0.83$ and $\rho=0.85$ for PRESBYOPIC, $\rho=0.85$ and $\rho=0.88$ for MYOPIC, $\rho=0.89$ and $\rho=0.98$ for TOP-DOwn, $\rho=0.7$ and $\rho=0.74$ for TOP. Similar trends appear for the connectedness, connectivity and communication cost.

Finally note that the topology with random selections of 3,4 or 5 children per node performs comparable to the topology with a fixed number of 4 children per node concluding that the effect of a random number of children per node is averaged.

\subsubsection{Adjusting the periodical executions}

Relative execution periods of the three levels in AETOS are crucial for the convergence speed and performance of self-organization. For example, an adaptation applied in the proximity view of the structuring level requires a period of time for updating the candidate parents and children. This is because the three levels are decentralized and interdependent within an overlay service. The update of the proximity view requires some gossip exchanges at the discovery level to collect new discovered samples. For this reason, the coordination level should not synchronize with its bottom levels. 
Table 4.4: Performance results of adaptation strategies for different tree topologies: (i) $k=3$, (ii) $k=5$ and (iii) $k=\operatorname{rand}(3,4,5)$. The values concern the (i) connectedness $\beta$, (ii) connectivity $\gamma$, (iii) fitness $\rho$ and (iv) the number of messages $\lambda$ (coordination level). Each pair of values refers to the 30th and 380th epoch of runtime.

\begin{tabular}{|c|c|c|c|c|}
\hline & PRESBYOPIC & MYOPIC & HUMBLE & GREEDY \\
\hline & \multicolumn{4}{|c|}{$k=3$} \\
\hline$\beta$ & $0.68,0.81$ & $0.99,1.0$ & $1.0,1.0$ & $0.67,0.85$ \\
\hline$\gamma$ & $0.92,0.94$ & $1.0,1.0$ & $1.0,1.0$ & $0.9,0.92$ \\
\hline$\rho$ & $0.83,0.83$ & $0.82,0.92$ & $0.83,0.85$ & $0.76,0.77$ \\
\hline \multirow[t]{2}{*}{$\lambda($ coordination level $)$} & 5116,5101 & 2478,2592 & 1968,1920 & 5737,5777 \\
\hline & \multicolumn{4}{|c|}{$k=5$} \\
\hline$\beta$ & $0.82,0.91$ & $1.0,1.0$ & $1.0,1.0$ & $0.84,0.88$ \\
\hline$\gamma$ & $0.93,0.96$ & $1.0,1.0$ & $1.0,1.0$ & $0.93,0.96$ \\
\hline$\rho$ & $0.84,0.85$ & $0.85,0.95$ & $0.84,0.88$ & $0.83,0.87$ \\
\hline \multirow[t]{2}{*}{$\lambda($ coordination level $)$} & 5587,5405 & 2430,2598 & 2327,2247 & 5575,5683 \\
\hline & \multicolumn{4}{|c|}{$k=\operatorname{rand}(3,4,5)$} \\
\hline$\beta$ & $0.78,0.92$ & $1.0,1.0$ & $1.0,1.0$ & $0.83,0.88$ \\
\hline$\gamma$ & $0.95,0.98$ & $1.0,1.0$ & $1.0,1.0$ & $0.95,0.95$ \\
\hline$\rho$ & $0.84,0.87$ & $0.83,0.92$ & $0.84,0.87$ & $0.81,0.83$ \\
\hline$\lambda($ coordination level) & 5313,5296 & 2557,2605 & 2212,2145 & 5643,5665 \\
\hline
\end{tabular}

\begin{tabular}{|l|c|c|c|c|}
\cline { 2 - 5 } \multicolumn{1}{c|}{} & \multicolumn{1}{c|}{ TOP-DOWN } & BOTTOM-UP & TOP & BОTтOM \\
\cline { 2 - 6 } \multicolumn{1}{c|}{} & \multicolumn{5}{c|}{$k=3$} \\
\hline$\beta$ & $0.31,0.99$ & $0.91,1.0$ & $0.33,0.56$ & $0.01,0.02$ \\
\hline$\gamma$ & $1.0,1.0$ & $1.0,1.0$ & $0.58,0.66$ & $0.84,0.84$ \\
\hline$\rho$ (coordination level) & $0.8,0.89$ & $0.65,0.65$ & $0.64,0.7$ & $0.86,0.86$ \\
\hline$\beta$ & 5830,5561 & 2,0 & 6751,6699 & 4329,4234 \\
\hline$\gamma$ & \multicolumn{5}{c|}{$k=5$} \\
\hline$\rho$ & $0.97,1.0$ & $0.5,1.0$ & $0.59,0.68$ & $0.02,0.02$ \\
\hline$\lambda$ (coordination level) & $1.0,1.0$ & $1.0,1.0$ & $0.66,0.72$ & $0.87,0.87$ \\
\hline$\beta$ & $0.92,0.98$ & $0.61,0.61$ & $0.69,0.74$ & $0.81,0.84$ \\
\hline$\gamma$ & 5708,5551 & 0,0 & 6411,6232 & 4881,4780 \\
\hline$\rho$ & \multicolumn{5}{|c|}{$k=\operatorname{rand}(3,4,5)$} \\
\hline$\lambda($ coordination level) & $0.86,0.97$ & $0.83,1.0$ & $0.46,0.6$ & $0.02,0.02$ \\
\hline
\end{tabular}

The experimental findings confirm this. If all levels are synchronized at $1000 \mathrm{~ms}$, the maximum average connectedness converges at 350th epoch for the GREEDY adaptation strategy. However, the convergence speed increases proportionally to the maximum performance for this adaptation strategy as the periodical execution of the discovery level and structuring level decreases to 500, 250, 200 and $100 \mathrm{~ms}$ at a cost of a proportional increase in the number of messages at these two levels. The number of messages exchanged by the coordination level also increases by approximately 600 messages in this range of periodical executions. This is because the proximity view is updated and therefore, there are available candidate parents and children that are contacted for the establishment of parent-child links. This trend is observed in the evaluation of all of the adaptation strategies. 


\subsubsection{The effect of T-MAN}

T-MAN enhances clustering at the structuring level and therefore plays a crucial role for the convergence speed of the performance metrics. For example, the connectedness achieved by the GREEDY adaptation strategy without T-MAN is $10 \%$ less and its convergence 200 epochs slower. Similarly, the fitness achieved with GREEDY by excluding T-MAN is $2 \%$ less and 150 epochs slower. Because of the slower update of the proximity view, the communication cost in the coordination level is lower by 500 messages in every epoch.

\subsection{Evaluation Summary}

The experimental results show that the TOP-DOwn $(\beta \approx 1, \gamma=1, \rho=0.95)$, HUMBLE $(\beta=1, \gamma=1, \rho=0.87)$ and MYOPIC $(\beta=1, \gamma=1, \rho=0.95)$ adaptation strategies achieve the highest performance with respect to connectedness $\beta$, connectivity $\gamma$, and fitness $\rho$. Convergence is achieved in fewer than 40 epochs except the fitness of TOP-DOwn and MYOPIC that converges within a minimum of 150 epochs. The communication cost that TOP-DOWN imposes to the coordination level of AETOS is 5400 messages per epoch and is the highest compared to HUMBLE and MYOPIC that require 2100 and 2600 messages per epoch respectively.

Adaptations of the proximity view positively influence connectedness, connectivity and fitness but negatively influence communication cost up to $50 \%$ compared to the case in which adaptations are not applied. Moreover, dynamic adoption of adaptation strategies, e.g., HYBRID-02 and HYBRID-03, shows that maximum connectedness, connectivity and fitness can be achieved at a minimum communication cost. Any single adopted adaptation strategy results in lower performance. Reseting adaptations is crucial to guarantee that the proximity view does not remain empty when the range of ranked nodes in the proximity view is constrained. A higher frequency of resets results in a small increase of communication cost, i.e., $2 \%$ for GREEDY. In addition, the results show a clear performance trend of AETOS in regards to the tree topology built: AETOS performs worse when a tree is built by a higher number of levels/lower maximum number of children $k$ compared to a tree with fewer number of levels/higher maximum number of children $k$. The performance difference for the connectedness, connectivity and fitness metrics can reach up to $12 \%$ between the cases of a 3-ary and a 5-ary tree.

Finally, when the frequency of the periodical executions in the coordination level decreases in relation to the periodical executions of the discovery level and structuring level, the convergence of the performance metrics is significantly faster at a cost of a proportionally higher communication rate in the discovery level and structuring level. Note that T-MAN also contributes significantly to the convergence speed of AETOS by, for example, a 200 epochs faster convergence for GREEDY.

\subsection{Comparison with Related Work}

In contrast to the application-level multicasting methodologies of Banerjee et al. [2003] and Wang et al. [2010] that are based on central components for managing or bootstrapping a tree overlay, AETOS is designed as an application-independent middleware 
for decentralized environments. Table 4.5 summarizes the related decentralized mechanisms discussed in this section. A more complete survey can be found in Pournaras [2009]. Note that the comparison is mainly qualitative. AETOS contributes a more generic, modular and reconfigurable self-organization of trees instead of performance enhancements.

Table 4.5: An overview of related decentralized mechanisms to AETOS.

\begin{tabular}{|c|c|c|c|c|}
\hline & Self-organization & Optimization & Reconfigurability & Applications \\
\hline AETOS & $\begin{array}{l}\text { gossiping, } \\
\text { clustering, } \\
\text { negotiation }\end{array}$ & $\begin{array}{l}\text { degree-bounding, } \\
\text { ordering, } \\
\text { balancing and } \\
\text { completeness }\end{array}$ & $\begin{array}{c}\text { three-level } \\
\text { modularity and } \\
\text { adaptation } \\
\text { strategies }\end{array}$ & generic \\
\hline Choe et al. [2004] & edge changes & $\begin{array}{l}\text { degree-bounding } \\
\text { and diameter } \\
\text { minimization }\end{array}$ & - & multicasting \\
\hline $\begin{array}{l}\text { Costa and Frey } \\
\text { [2005] }\end{array}$ & $\begin{array}{l}\text { DHT mapping } \\
\text { and breadth-first } \\
\text { traversing }\end{array}$ & degree-bounding & - & $\begin{array}{c}\text { content-based } \\
\text { publish-subscribe } \\
\text { systems }\end{array}$ \\
\hline $\begin{array}{l}\text { England et al. } \\
\text { [2007] }\end{array}$ & $\begin{array}{l}\text { minimum } \\
\text { spanning tree } \\
\text { algorithms, e.g., } \\
\text { Bellman-Ford } \\
\text { algorithm }\end{array}$ & $\begin{array}{l}\text { hop counting, } \\
\text { path weighting }\end{array}$ & $\begin{array}{l}\text { trade-offs by } \\
\text { weighted } \\
\text { optimization } \\
\text { metrics }\end{array}$ & $\begin{array}{l}\text { sensor networks } \\
\text { and load } \\
\text { scheduling }\end{array}$ \\
\hline $\begin{array}{l}\text { Fei and Yang } \\
\text { [2007] }\end{array}$ & $\begin{array}{l}\text { minimum } \\
\text { spanning tree } \\
\text { algorithms, e.g., } \\
\text { Prim's algorithm }\end{array}$ & $\begin{array}{l}\text { degree-bounding, } \\
\text { proactive recovery }\end{array}$ & $\begin{array}{l}\text { NAT } / \text { firewalls, } \\
\text { grandfathers- } \\
\text { siblings and } \\
\text { uncles- } \\
\text { granduncles } \\
\text { candidates }\end{array}$ & multicasting \\
\hline $\begin{array}{l}\text { Frey and Murphy } \\
\text { [2008] }\end{array}$ & $\begin{array}{c}\text { negotiation } \\
\text { protocol and cycle } \\
\text { prevention }\end{array}$ & degree-bounding & repair strategies & generic \\
\hline $\begin{array}{l}\text { Jagadish et al. } \\
{[2005,2006 b]}\end{array}$ & $\begin{array}{c}\text { join at first } \\
\text { available bottom } \\
\text { node }\end{array}$ & balancing & $\begin{array}{c}\text { network } \\
\text { restructuring and } \\
\text { load-balancing }\end{array}$ & $\begin{array}{c}\text { multi-dimensio- } \\
\text { nal data indexing, } \\
\text { exact and range } \\
\text { queries }\end{array}$ \\
\hline $\begin{array}{l}\text { Leitao et al. } \\
{[2007]}\end{array}$ & $\begin{array}{l}\text { pull and lazy } \\
\text { push gossiping } \\
\text { strategies }\end{array}$ & hop counting & $\begin{array}{l}\text { threshold-based } \\
\text { optimization and } \\
\text { gossiping } \\
\text { parameters }\end{array}$ & broadcasting \\
\hline Tan et al. [2005a] & $\begin{array}{c}\text { leaf joins and } \\
\text { sift-up operations }\end{array}$ & $\begin{array}{l}\text { bandwidth and } \\
\text { time based } \\
\text { ordering }\end{array}$ & $\begin{array}{c}\text { frequency of } \\
\text { sift-up operations }\end{array}$ & live streaming \\
\hline Tang et al. [2005] & $\begin{array}{c}\text { DVMRP }^{\mathrm{b}} \\
\text { mechanism over } \\
\text { random/proximi- } \\
\text { ty-based } \\
\text { gossiping }\end{array}$ & $\begin{array}{c}\text { degree-bounding, } \\
\text { latency }\end{array}$ & $\begin{array}{l}\text { dynamic } \\
\text { periodical } \\
\text { executions }\end{array}$ & $\begin{array}{c}\text { group } \\
\text { communication }\end{array}$ \\
\hline $\begin{array}{l}\text { Yuan and Wei } \\
\text { Tsang [2004] }\end{array}$ & $\begin{array}{l}\text { mesh-first } \\
\text { protocol and } \\
\text { top-down } \\
\text { construction }\end{array}$ & $\begin{array}{c}\text { minimum } \\
\text { spanning and } \\
\text { shortest path tree }\end{array}$ & $\begin{array}{l}\text { timer for parent } \\
\text { selection }\end{array}$ & video streaming \\
\hline
\end{tabular}

\footnotetext{
a Network Address Translation.

${ }^{\mathrm{b}}$ Distance Vector Multicast Routing Protocol.
} 
Related approaches follow a different design approach in which self-organization and tree optimization are designed based on the requirements of a specific application type or domain. Tan et al. [2005a] introduce a combination of a bandwidth-ordered and time-ordered tree overlay for streaming applications. The position of each node is continuously evaluated and improved by calculating the 'service capability contribution' (SCC). This metric is the product of the outbound bandwidth of a peer and its age (uptime) in the system. Based on the SCC metric, nodes are sorted by shifting parentchildren positions. In contrast, the nodes of AETOS are ranked with an abstract weight value attributed by the utilization level responsible for the abstraction of application criteria to application-independent weights of nodes. Furthermore, the SCC of Tan et al. [2005a] is computed as the product of two independent metrics. This would not be the case if more metrics with interdependencies are combined. For example, latency experienced by peers may be related to their available bandwidth. Therefore, the two metrics have a dependency that makes the estimation of a latency-aware SCC challenging. More knowledge about the underlying network infrastructure is required and therefore more complexity in self-organization. This is the reason that AETOS decouples application complexity from self-organization. The utilization level should be realized to handle this complexity by associating the weights of the nodes with the performance criteria for a specific application type.

One important aspect of comparison is the optimization of the topology self-organization. For example, the approaches of Leitao et al. [2007], Yuan and Wei Tsang [2004], Choe et al. [2004] and Tan et al. [2005b] are limited to building a minimum spanning tree known to minimize latency in multimedia multicasting applications. However, these approaches do not adopt any ordering process of nodes that could express the heterogeneity of their performance profiles. AETOS does not explicitly optimize trees for a given underlying network infrastructure. Nevertheless, it can provide such optimization if it is instructed appropriately by the utilization level. Similar issues concern tree overlays designed for optimizing distributed database queries, e.g., range queries. In this case, trees act as indexing structures. Load-balancing for minimizing the computational load of peers is the optimization applied in this application type by Jagadish et al. [2005], and Jagadish et al. [2006b]. However, note that this optimization is not relevant for the time-ordered tree of Tan et al. [2005a] as it results in a load-imbalanced tree. Different applications require different graph properties in the formed tree overlay networks. The same concept holds for trees that serve publish-subscribe systems [Costa and Frey, 2005]. In contrast, AETOS achieves different topological properties in the formed trees transparently from applications. This is achieved by changing the organizational criteria of the utilization level and adopting a different adaptation strategy.

AETOS combines proactive and reactive features in its design concerning the resilience of the tree self-organization. Proactiveness is related to the (i) ordering of the tree to minimize, for example, the impact of peer failures and (ii) the dynamic maintenance of the random view and proximity view. These proactive features support and serve the reactiveness of AETOS: The feedback provided by the coordination level and the adaptation strategies that manage this feedback to improve self-organization and meet the organizational goal. The combination of proactiveness and reactiveness is one of the novel properties of AETOS. In contrast, the following related methodologies introduce an explicit proactive or reactive mechanism for building tree overlays for a specific application. Proactive mechanisms may come with a high communication and computational cost. Fei and Yang [2007] introduce a highly proactive method for main- 
taining a minimum spanning tree for multicasting. Alternative back-up parents are negotiated before departures of peers occur. Therefore, reconnections are rapid and the tree becomes more resilient. However, the benefit of this high responsiveness comes with a high bandwidth consumption. This proactive method pays back the high bandwidth cost in case of frequent peer departures. If peer departures are rare, the system experiences a significant bandwidth overhead without an actual benefit in the resilience of the tree. Alternative proactive methodologies for tree resilience are evaluated by Birrer and Bustamante [2007]. These methodologies are based on various schemes of redundancy: (i) cross-link, (ii) in-tree and (iii) multiple-tree redundancy. However, redundancy significantly increases the communication cost and the resources of peers. It is also illustrated as a relevant solution for multicasting applications. Finally, proactive and reactive components of the epidemic broadcast trees introduced by Leitao et al. [2007] work in synergy to provide a fast tree construction and repair. However and in contrast to AETOS, the proximity of nodes is not considered and the formed trees are not ordered to express the heterogeneity of peers and their applications.

Most other related approaches [Tan et al., 2005a, Costa and Frey, 2005, Walters et al., 2008, Jagadish et al., 2006b] are based on adaptation without the use of proactive back-end mechanisms. Frey and Murphy [2008] propose a number of transformation and rewiring strategies for reconnecting disconnected branches. The configuration of tree connections is similar to the configuration which the coordination level of AETOS performs. However, the adaptations of Frey and Murphy [2008] do not sort a tree or control its graph properties required for an application.

AETOS is highly customizable as it is based on the generic and application-independent ASMA architecture, composed of three continuously reconfigurable levels and several adaptation strategies. The application-dependence of most of the related approaches unavoidably results in a low customization and optimization options. Some of these approaches [England et al., 2007, Jagadish et al., 2006b, Leitao et al., 2007] introduce additional costly protocols and management mechanisms to improve the customization of self-organization in dynamic scenarios. For example, custom tree repairing, load-balancing and decentralization mechanisms are some of them. Furthermore, the periodical executions in the protocols of Leitao et al. [2007], Tang et al. [2005] and Tan et al. [2005a] are limited to control or influence the convergence speed and the consumed bandwidth in application-level multicasting. In contract, by customizing inter-related periodical executions of the three levels of AETOS, performance is tuned as shown in Section 4.7.2.

Exploration of multiple strategies for self-organization of trees is significantly limited in related work. Frey and Murphy [2008] illustrate six repair strategies for trees. These strategies are related to the satisfaction of application requirements, e.g., node degrees, during parent-child reconnections in the tree. These strategies appear to influence the tree topology in a similar way as the adaptation strategies of AETOS, for example, forming lower or higher branches. However, compared to AETOS, there are two fundamental differences: (i) The AETOS agents always respect the organizational criteria, such as the maximum number of children. (ii) The adaptation strategies do not only concern the tree repairs, but both the building and maintenance of trees. Therefore, the adaptation strategies are integrated to a higher degree in the core self-organization of AETOS. 


\subsection{Discussion and Future Work}

Ranking nodes with numerical weights that abstract one or more blended application criteria is possible for a wide range of application types as shown in Section 4.1 and 4.9 but not for every type. There could be several problems in ranking. Weights should be independent meaning that if the weight of a node depends on the weights of other nodes there is no linearity in the sorting process and therefore weight comparisons are biased. Bahl et al. [2006] deal with this problem. Furthermore, several criteria are not measurable or composed. For example, the availability or bandwidth of peers as an application criterion is a measurable and comparable metric. However security is not straightforward to measure and compose as illustrated by Müller-Quade and Renner [2009]. Note that the weighting scheme of AETOS concerns only the nodes and not the links between them. Various performance metrics deal with the links and not only the nodes such as the latency in application-level multicasting. All of these issues are interrelated and should be addressed in future work.

The weights of the nodes in the performed experiments are static, meaning that they do not change during runtime. In case this assumption does not hold, there are various approaches to satisfy the consistency of the tree ordering. For example, if changes of the weights are synchronous, meaning that all weights change at the same time point, self-organization process may be reinitiated. If changes of the weights are asynchronous and infrequent, the affected parent-child connections may be simply dropped and the coordination level of AETOS must guarantee that the new connections are coordinated based on the up-to-date weights. If dropping connections is not a desirable approach, alternative reactive mechanisms such as the one of Frey and Murphy [2008] may be applicable. Another solution is to use the 'healer' peer selection policy of the peer sampling service in the discovery level [Jelasity et al., 2007]. This policy considers the age the peers in the system. If the age represents the most recent weight of a node in the system, then the discovery level disseminates and collects the most recently updated weights resulting in more consistent parent-child connections. Finally, if changes are highly frequent, weight averaging may by unavoidable to deal with the extreme communication cost that frequent adaptations originate in the self-organization process. Failures of peers can be handled based on similar concepts [Vassilakis and Stavrakakis, 2010].

Adaptation strategies provide a level of abstraction, modularity and customization in AETOS. Furthermore, they hide a significant level of detail on how the adaptations in self-organization are performed. This results in a lower complexity at the coordination level and a higher transparency than manual system parameterizations. Performance trade-offs can be explored using the adaptation strategies. For example, the TOP-DOwN adaptation strategy provides the highest fitness by consuming a large number of messages. In contrast, MYOPIC achieves a comparable fitness by consuming fewer than half of the messages of TOP-DOwn. Similar performance trade-offs can be explored by enabling or disabling the adaptations in the proximity view. Dynamic adoption of adaptation strategies during runtime results in hybrid adaptation strategies that collectively achieve a higher performance than the individual adaptation strategies of which they are composed of. Emergent adoption of adaptation strategies and reasoning is part of future work.

Adaptation strategies do not depend on the coordination level that is agnostic of the built topology. Other types of topologies can be built if they are organized based on the proximity of their nodes. Super-peer topologies [Yang and Garcia-Molina, 2003, 
Pournaras et al., 2008a] or distributed hash tables [Kaashoek et al., 2003] are examples of such topologies.

The discovery level and structuring level generate a significant number of messages, yet constant and controlled by the periodical gossiping executions. Note that these two levels are generic and therefore their communication cost can be shared between different applications. For example, the peer sampling service maintains a highly connected overlay network that could be used as a back-end for different systems. If the computational or other resources of a host cannot support every level of AETOS, the peer could provide a virtualization by deploying each level of AETOS on different hosts.

\subsection{Conclusions}

This chapter concludes that AETOS is able to build and maintain complex self-organizing tree topologies defined by several graph properties related to a wide range of application requirements. Our experimental findings confirm the cost-effectiveness of AETOS by introducing and measuring a wide range of generic performance metrics applicable for various applications. Self-organization is designed as an overlay service in contrast to other related methodologies that (i) provide a dedicated mechanism for an application domain and (ii) study a subset of the graph properties introduced in this chapter. The separation of organizational complexity in different levels that follow the ASMA architecture proves to be a modular and customizable design choice for this overlay service. The adaptation strategies result in different tree topologies by exploring different performance trade-offs. The adaptation strategies can also be combined to compose hybrid adaptation strategies with collective properties suggesting a new meta-level of abstraction and reasoning in future work. 


\title{
Chapter 5

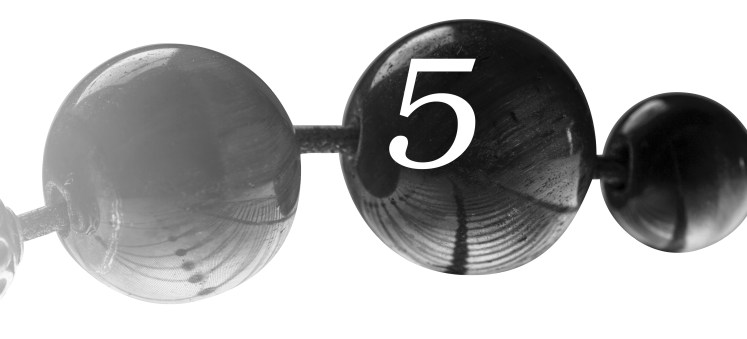

\section{The Dynamic Intelligent Aggregation Service}

\begin{abstract}
"These (elements) never cease changing place continually, now being all united by Love into one, now each borne apart by the hatred engendered of Strife, until they are brought together in the unity of the all, and become subject to it."
\end{abstract}

Empedocles $^{1}$

The increasing scale and decentralization of complex distributed systems and applications results in an information gap: Agents, with partial knowledge about a system, require the local availability of collective and summarized knowledge about the state of the whole system to perform decision-making, adapt the execution of their tasks and meet global application objectives. Therefore, aggregation of information becomes a crucial requirement to acquire such collective and summarized knowledge for a wide range of distributed applications. For example, aggregation is used to locate paths of trustworthy service providers as illustrated by Wang and Singh [2006]. Aggregation is widely used as a core operation for managing and reducing the energy consumption in sensor networks. Baek et al. [2004] and Boulis et al. [2003] study aggregation in this application domain. Aggregation for the stabilization of energy consumption [Pournaras et al., 2010c, 2009b] in Smart Power Grids is investigated in Chapter 6.

Centralized computation of aggregation functions is straightforward as the whole set of information is available in one location. However, centralized aggregation is not always an option for reasons that may concern scalability or privacy. This chapter focuses on the challenging problem of decentralized aggregation of information distributed across the peers of a network. Aggregates are computed locally by agents in every peer in a network ${ }^{2}$. Communication, storage and processing costs are fundamental issues

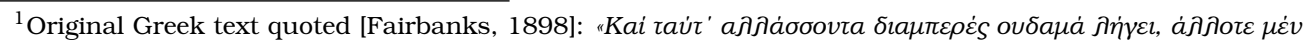

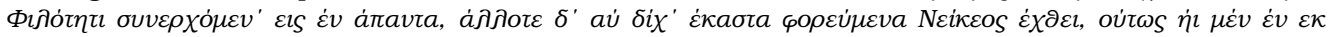

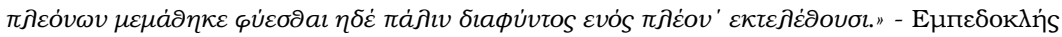

${ }^{2}$ Note that the result of these computations actually represents estimations of aggregates as distributed environments are typically not deterministic.
}

"This chapter is based on a submitted journal paper [Pournaras et al., 2013a]. 
that challenge the design of a generic overlay service for decentralized aggregation.

This chapter introduces DIAS, the Dynamic Intelligent Aggregation Service to address the following four aspects and limitations of decentralized aggregation:

- Lack of abstraction in the computation of different aggregation functions. Different methodologies [Jelasity et al., 2005, Kempe et al., 2003, Nath et al., 2008] have been introduced for specific aggregation functions or classes of functions as a result of this drawback.

- Lack of abstraction in the use of different routing mechanisms. Most aggregation methodologies [Jelasity et al., 2005, Kashyap et al., 2006, Kempe et al., 2003] are actually information routing mechanisms for the computation of specific aggregation functions, e.g., tree topologies or information diffusion.

- Lack of adaptations in computed aggregates when agent information changes. Related methodologies [Jelasity et al., 2005, Kashyap et al., 2006, Kempe et al., 2003, Nath et al., 2008] either assume static information or perform expensive recomputations.

- Influence in accuracy in case of duplicate information [Haridasan and van Renesse, 2008] routed over an unstructured (irregularly organized) overlay network or when the local information of the agents changes [Nabeel Ahmed, David Hadaller, 2006].

DIAS is an overlay service designed according to the ASMA architecture illustrated in Chapter 3. It is realized as a generic, agent-based and decentralized middleware for dynamic aggregation based on a holistic approach: availability of distributed information in every peer of the network that enables the simultaneous computation of almost any aggregation function. Multi-level discovery, classification and aggregation of the distributed information in the network, as defined by ASMA, make this holistic approach possible.

DIAS is based on the concept of aggregation membership. Aggregation memberships are aggregation information derived and abstracted from the explicit distributed aggregation values. For example, an agent has memberships of other agents whose information is aggregated. Complementarily, an aggregate of an agent has memberships of the aggregated information in other agents. This chapter shows that such implicit information can be locally and efficiently stored in probabilistic data structures known as the bloom filters [Bloom, 1970, Brodera and Mitzenmacher, 2004, Dillinger and Manolios, 2004].

A known problem of bloom filters is that of false positives [Bloom, 1970]. A false positive incorrectly denotes that a membership exist when it actually does not. DIAS is able to detect inconsistencies such as duplicate and outdated information under the effect of false positives generated by bloom filters. This chapter shows how detection is possible by mutually checking the memberships between the remote agents of DIAS without introducing additional communication. Experimental evaluation illustrates the efficiency and performance trade-offs of DIAS. High accuracy is achieved under a range of aggregation and resource-constrained settings.

This chapter is outlined as follows: Section 5.1 illustrates the problem description in depth. Section 5.2 outlines the architecture of DIAS based on the ASMA architecture. 
Section 5.3 introduces the model of dynamic aggregation in DIAS. Section 5.4 shows the realization of the discovery level in DIAS. Section 5.5 illustrates the classification process in the structuring level of DIAS based on the concept of aggregation memberships. Section 5.6 outlines the computation of aggregates in the coordination level of DIAS. Section 5.7 follows with a realization of the aggregation memberships based on the probabilistic data structures of bloom filters. Section 5.8 evaluates the performance of DIAS under various experimental settings. Section 5.9 summarizes this evaluation. Section 5.10 compares DIAS with related methodologies. Section 5.11 discusses the approach of DIAS and outlines future work. Finally, Section 5.12 concludes this chapter.

\subsection{Problem Description}

Assume an overlay network of nodes, all having an aggregation value representing the state of a (application) parameter. These nodes acquire the computation of a set of aggregates $^{3}$. Decentralized aggregation is defined in this chapter as the computation of aggregation functions (aggregates) by all of the nodes of an overlay network that have as an input the total aggregation values of all the nodes in this overlay network.

A generic overlay service for aggregation should perform decentralized aggregation for a wide range of distributed applications. This is challenging as most distributed aggregation systems experience the following four limitations:

1. Function-dependence: Distributed applications may require the computation of a wide range of aggregation functions. Average, summation, MAXIMUM and Minimum are common numerical aggregation functions. Textual and rule aggregation are more complex. Aggregation functions share different mathematical properties as shown by Calvo et al. [2002] and, therefore, their computational requirements may vary significantly. Due to this reason, different aggregation methodologies have been developed for specific aggregation functions or classes of aggregation functions. Most existing aggregation methodologies are function-dependent and have limited applicability.

For example, Jelasity et al. [2005] propose an aggregation methodology based on gossiping. This methodology calculates the AVERAGE function as an iterative variance reduction algorithm over the values of nodes in an overlay network. Nonetheless, the counT operator that estimates the number of participating nodes cannot be calculated without additional protocol complexity. The sumMATION operator is derived by the product estimation of AVERAGE and COUNT and therefore, two instances of gossiping protocols are required. Similar issues are raised by Kempe et al. [2003] together with inaccuracy issues when there are failures in the network. Note that although gossiping protocols provide highly robust information routing, robustness in the accuracy of aggregation is not guaranteed.

2. Routing-dependence: The variance reduction algorithm applied in the aggregation approach of Jelasity et al. [2005] requires gossiping communication between peers in a network. Information diffusion based on which distributed aggregation is performed in the approach of Kempe et al. [2003] also depends on a similar gossiping

\footnotetext{
${ }^{3}$ Aggregates should be relevant to the distributed aggregation values. This means that the information type of the aggregation value, e.g. numerical or textual, matches with the input information type of the aggregation functions that compute these aggregates.
} 
protocol. Aggregation over structured topologies, such as trees, relies on multicasting. Replacing the routing mechanism of an aggregation methodology with a different one can make aggregation cost-ineffective if not totally infeasible and inaccurate. For example, tree aggregation requires unique paths between nodes in an overlay network to avoid double-counting. This requirement is not satisfied in unstructured (random) overlay networks maintained by gossiping protocols. Therefore, most aggregation methodologies are designed in line with the properties, strengths and constraints of the routing mechanism selected to support them.

3. Static aggregation values: The aforementioned gossiping and tree aggregation methodologies assume that aggregation values in nodes do not change during aggregation. However, these values may change and be derived from a continuous or discrete domain of values. Speed of change matters. Distributed aggregation schemes may be infeasible if aggregation values are highly dynamic. Investigating the degree of tolerable changes in the aggregation values of nodes is crucial for a dynamic aggregation system. Updating aggregates with new aggregation values is potentially a more cost-effective solution than performing expensive recomputation.

4. Inaccuracies: Inaccuracies are estimations of aggregates with significant deviation from the actual aggregates. Two types of inaccuracies are studied: (i) doublecounting and (ii) outdated aggregation values, i.e., values that have changed during runtime. In duplicate-sensitive aggregation functions, such as summation, summing aggregation values twice results in an inaccurate aggregate. The same holds if aggregation values of nodes in an overlay network change dynamically during system runtime. Aggregates require adaptation to converge to their most recent actual values. Other inaccuracies related to network uncertainties, faultintolerance etc. are not the focus of this thesis and are usually related to the adopted routing mechanism [Kennedy et al., 2009].

The above limitations appear to a certain degree in most of the existing aggregation approaches of Nabeel Ahmed, David Hadaller [2006], Artigas et al. [2006], Haridasan and van Renesse [2008], Jelasity et al. [2005], Kashyap et al. [2006], Kempe et al. [2003], Nath et al. [2008] and are mentioned in the related surveys of Chitnis et al. [2008], Fasolo et al. [2007], Kennedy et al. [2009], Rajagopalan and Varshney [2006], Sang et al. [2006] and Weise et al. [2008]. These approaches and their limitations are discussed and compared in detail in Section 5.10. Motivated by these issues, this chapter focuses on the problem of designing an efficient overlay service for dynamic, accurate and decentralized aggregation decoupled from a specific routing mechanism and aggregation function.

\subsection{System Overview}

This chapter introduces DIAS, the Dynamic Intelligent Aggregation Service. DIAS is a middleware overlay service that computes aggregation functions from an input set of continuously changing aggregation values distributed in every peer of a network. Figure 5.1 illustrates the architecture of DIAS as an overlay service realization of ASMA. 


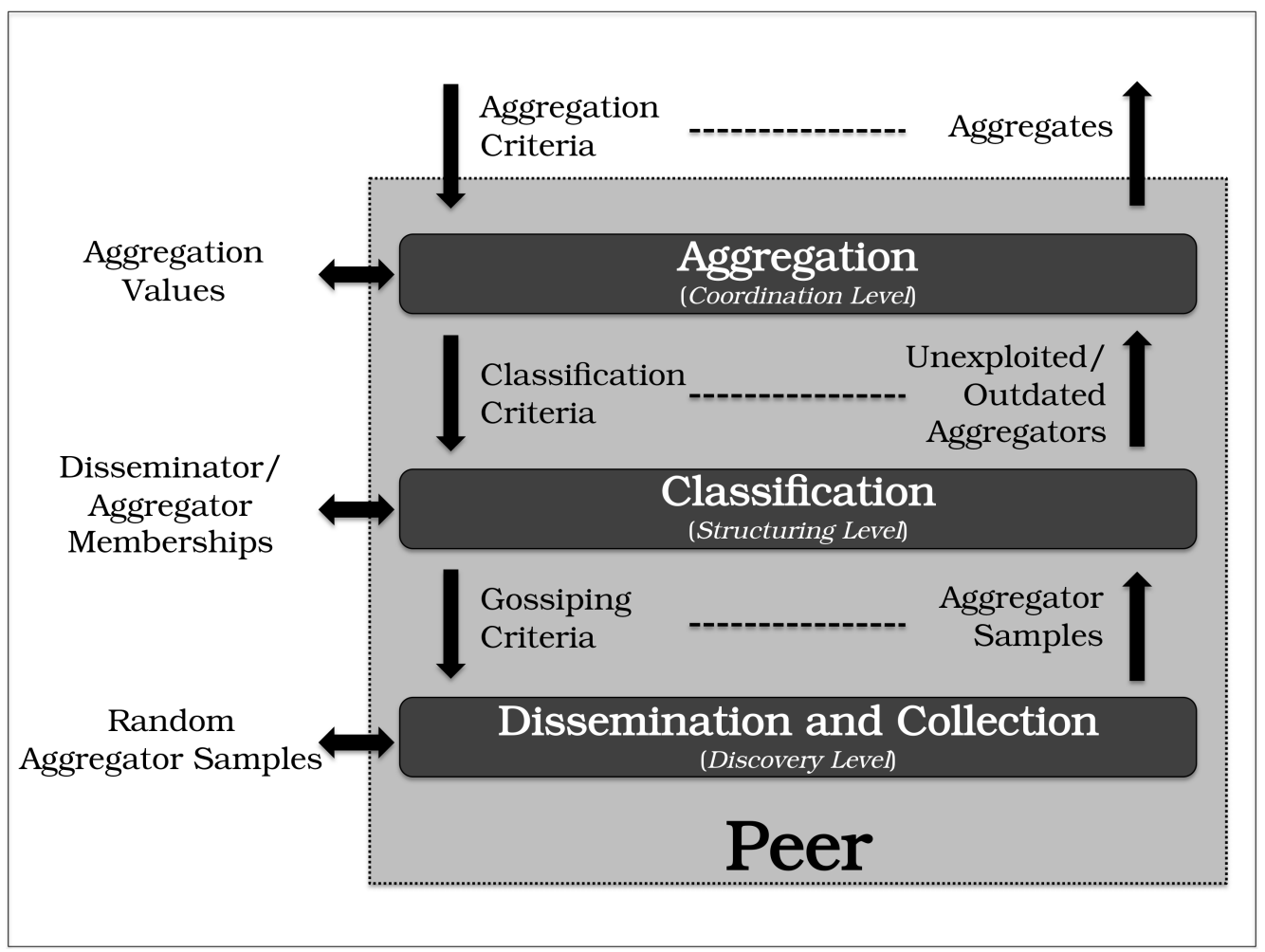

Figure 5.1: The DIAS middleware architecture. The architectural levels, criteria and samples realize the ones defined in ASMA as illustrated in Figure 3.1

Agents, as defined in Section 2.3, represent the three levels of DIAS and act as aggregators and disseminators. These three agents, within a peer, provide aggregation values to the agents of other peers and consume aggregation values from them. This setting is the most challenging in matter of performance as the maximum communication, storage and computational cost is experienced by every peer in the network. However, note that in practice, applications may not require all agents to disseminate and aggregate values. Section 5.11 discusses this issue in more detail.

The discovery level ${ }^{4}$ of DIAS is responsible for gossip-based dissemination and collection of the aggregator samples. Disseminators gossip aggregator samples to locate the agents to which the aggregation values need to be sent. Gossiping can be continuously parameterized by gossiping criteria as illustrated in Section 3.1.2.

The discovered aggregator samples are provided to the structuring level in which they are classified. Each disseminator classifies the received aggregators into three possible classes: (i) exploited, (ii) unexploited and (iii) outdated. These classes indicate if the aggregation value of a disseminator has been aggregated before by the classified aggregators, if it has not been aggregated or if an earlier (outdated) aggregation value has been aggregated that has changed. Classification is performed based on histori-

\footnotetext{
${ }^{4}$ The discovery level of DIAS, similarly to AETOS, uses the gossiping mechanism of the peer sampling service [Jelasity et al., 2007].
} 
cal aggregation information that indicates membership of an aggregation value in the computed aggregates. Unique membership of an aggregation value in aggregates is the minimum information required to decide if this value is new, outdated or duplicate in aggregation. Memberships are the result of remote interactions between remote agents of the structuring level. The structuring level provides to the coordination level contact information of possible aggregators to which aggregation values can be aggregated. DIAS is able to tune discovery of new aggregation values instead of updating the existing aggregated values and the other way around. These are the adaptation strategies of DIAS and are configured by the classification criteria provided by the coordination level.

Finally, the coordination level interacts with the remote aggregators to exchange aggregation values. These overlay interactions have two possible semantics: exploitation of a new aggregation value or update of aggregates with the most recent aggregation value. A number of aggregates are computed and delivered to the applications as defined by the aggregation criteria.

DIAS addresses the limitations illustrated in Section 5.1 at a cost of higher communication overhead compared to related methodologies that specialize in specific aggregations functions or routing mechanisms [Jelasity et al., 2005, Nath et al., 2008]. As most of these limitations are related to a lack of abstraction, modularity and customization of the aggregation mechanisms, DIAS is designed based on the ASMA architecture that splits the complexity of dynamic decentralized aggregation into three self-organization levels.

Memberships of DIAS are the means to detect inaccuracies such as double-counting and outdated aggregation values. However, a decentralized system cannot explicitly store memberships of all aggregation values locally in each peer. This approach is neither scalable, efficient nor decentralized. To overcome this challenge, this chapter introduces probabilistic data structures for management of memberships, bloom filters, illustrated by Brodera and Mitzenmacher [2004]. Bloom filters provide tremendous space savings at a cost of false positive memberships. DIAS, however, is able to detect false positive inconsistencies and, therefore, maintain a high accuracy level in the computed aggregates without introducing additional communication cost.

The routing requirements of DIAS are restricted to information dissemination and information collection as shown in Figure 5.1. The discovery level of ASMA can be realized with different mechanisms as discussed in Section 3.1.2. DIAS computes aggregation functions based on efficient local availability of all of the dynamically changing aggregation values in the network. If aggregators can locate the whole sample of aggregation values efficiently, different aggregation functions can be computed regardless of the data type stored, e.g., numerical or textual.

\subsection{Modeling of Dynamics}

This section introduces a model for aggregation of states. A state represents a (aggregation) value of an application parameter at a specific point in time. The state of an application parameter changes during runtime. Decentralized aggregation computes aggregation functions that receive as input the states of different peers. All aggregated states in each peer correspond to the same application parameter.

Assume that $n$ peers of DIAS form an aggregation overlay network of $n$ nodes. Each 
node $i$ contains an aggregator $\mathrm{A}_{i}$ and a disseminator $\mathrm{D}_{i}{ }^{5}$. Each of these nodes contains a selected state $s_{i}^{\prime}$ that is the one to be aggregated in all nodes. During each runtime iteration, selected state $s_{i}^{\prime}$ can be equal to one and only one state from a finite number $v$ of locally unique possible states $s_{i}^{\prime}=s_{i}^{0}\left|s_{i}^{1}\right| \ldots \mid s_{i}^{v-1}$. For example, in a movie recommender system, movies are ranked with one to five stars. The number of stars are the possible states and an actual ranking of a movie is the selected state. Note that two possible states in different nodes may have the same value but not in a single node. As the selected state changes, an earlier selected state is indicated as $\hat{s}_{i}$.

The system goal is the aggregation $f\left(s_{0}^{\prime}, s_{1}^{\prime}, \ldots, s_{n-1}^{\prime}\right)$ of all of the selected states in the overlay network during an aggregation phase. An aggregation phase is defined as the time period in which the selected states may change but the set of possible states remains the same. During an aggregation phase, the aggregates change continuously as a result of changes in the local selected states. Aggregation does not converge to a single value but rather to a distribution of aggregates over time.

Section 5.11 discusses the applicability of this model in distributed applications.

\subsection{Dissemination and Collection}

Decentralized aggregation requires the means to access all of the locations of aggregators that acquire the selected states of disseminators. Dissemination and collection of aggregator samples via gossiping provide lookup in a distributed environment. An aggregator sample contains the network peer identifier of this aggregator, e.g., IP address and port number. Each agent of the discovery level maintains a random view of size $r$ with aggregator samples that are continuously updated via the gossiping protocol of the peer sampling service [Jelasity et al., 2007]. The realization of the discovery level is illustrated in Section 3.1.2.

Introducing a highly connected and dynamic overlay network provides higher availability of the aggregation values. Furthermore, continuous update of the random view enables the discovery of aggregation values that have changed.

\subsection{Consistent Aggregation Sessions}

The purpose of the structuring level in DIAS is to provide aggregators to the coordination level that guarantee consistent aggregation sessions.

An (unidirectional) aggregation session concerns (re)computation of the aggregates by an aggregator $A_{j}$ after the receipt of a selected state from a remote disseminator $D_{i}$. If (re)computation occurs in both aggregators of nodes $i$ and $j$, this aggregation session is bidirectional.

An aggregation session is consistent if the input selected state of performed (re)computation by an aggregator $A_{j}$ is not (i) a duplicate or (ii) an outdated selected state that has now changed. A consistent aggregation session between an aggregator $A_{j}$ and a disseminator $D_{i}$ is mutually satisfied if and only if the following conditions hold:

- The disseminator $D_{i}$ disseminates for first time (i) its selected state, or (ii) its updated selected state to the aggregator $\mathrm{A}_{j}$.

\footnotetext{
${ }^{5}$ Recall from Section 5.2 that this is the most challenging performance setting resulting in the maximum communication, storage and computational complexity.
} 
- The aggregator $\mathrm{A}_{j}$ aggregates for first time (i) the selected state, or (ii) the updated selected state of the disseminator $\mathrm{D}_{i}$.

An inconsistent aggregation session usually results in inaccurate aggregates. Note that double-counting does not always result in inaccuracies as some aggregation functions are insensitive to duplicates, i.e., MAXIMUM or MINIMUM. However, duplicates cause additional communication and processing overhead in hosts. For this reason, this chapter treats inconsistent aggregation sessions as subject of prevention.

Selecting aggregators that result in consistent aggregation sessions requires some form of history information about the past aggregation sessions performed. This section introduces the concept of aggregation memberships and their use to classify aggregators in the outdated, exploited and unexploited classes. Beyond consistency, this classification provides the option to perform the update of aggregates in favor of (i) changing (outdated) aggregation values or (ii) unexploited aggregation values. These two options distinguish the two adaptation strategies of DIAS.

\subsubsection{Aggregation memberships}

This section illustrates the concept of aggregation membership. Assume a sample of aggregation values that each becomes an input in an aggregation function during an aggregation phase. If an arbitrary aggregation value is selected from the whole sample at an arbitrary time point during this aggregation phase, this aggregation value has a probability of membership in the computed aggregates. Aggregation membership $M_{\text {group }}$ (member) of a certain 'member' to a certain 'group' is either positive or negative.

This concept can be applied to the aggregation dynamics illustrated in Section 5.3. Each agent of the structuring level in a peer $i$ stores unique identifiers of possible states $\mathrm{S}_{i}^{0}, \ldots, \mathrm{S}_{i}^{v-1}$ corresponding to the actual possible states $s_{i}^{0}, \ldots, s_{i}^{v-1}$. Respectively, $\mathrm{S}_{i}^{\prime}$ and $\hat{S}_{i}$ refer to the unique identifiers of the selected $s_{i}^{\prime}$ and outdated $\hat{s}_{i}$ state in peer $i$. The structuring level stores a representation of the local states, their unique identifiers, and the coordination level stores the actual states, e.g., numerical or other type. The structuring level also uses the local unique peer identifier to map the local aggregator $\mathrm{A}_{i}$ and disseminator $D_{i}$. Therefore, $A_{i}=D_{i}$. The following four aggregation memberships are defined in a unidirectional aggregation session between an aggregator $A_{j}$ and a disseminator $\mathrm{D}_{i}$ in two peers $i$ and $j$ :

Membership $1\left(M_{D_{i}}\left(A_{j}\right)\right)$. Membership of an aggregator in a disseminator.

A disseminator $D_{i}$ stores the identifier of an aggregator $A_{j}$ to which it has disseminated its selected state at least once during an aggregation phase.

Membership $2\left(M_{S_{i}^{u}}\left(\mathrm{~A}_{j}\right)\right)$. Membership of an aggregator in a possible state.

A disseminator $D_{i}$ stores the identifier of an aggregator $A_{j}$ for each possible state identified as $S_{i}^{u}$ aggregated by this aggregator.

Membership $3\left(M_{\mathrm{A}_{j}}\left(\mathrm{D}_{i}\right)\right)$. Membership of a disseminator in an aggregator.

An aggregator $A_{j}$ stores the identifier of a disseminator $D_{i}$ from which it has aggregated its selected state at least once during an aggregation phase.

Membership $4\left(M_{A_{j}}\left(\mathrm{~S}_{i}^{\prime}\right)\right)$. Membership of a selected state in an aggregate. 
An aggregator $A_{j}$ stores the identifier of a selected state $S_{i}^{\prime}$ aggregated from a disseminator $D_{i}$.

Figure 5.2 illustrates the aggregation memberships of DIAS stored in the structuring level. Aggregation memberships can be used as follows: Assume an aggregation session between disseminator $D_{i}$ that sends its selected state $S_{i}^{\prime}$ and aggregator $A_{j}$ that receives this state. Disseminator $D_{i}$ knows if aggregator $A_{j}$ aggregates its selected state $S_{i}^{\prime}$ for first time by checking aggregation membership $M_{D_{i}}\left(A_{j}\right)$. Furthermore, $D_{i}$ knows if $A_{j}$ has aggregated a different possible state earlier by checking all aggregation memberships $M_{S_{i}^{u}}\left(\mathrm{~A}_{j}\right)$. Respectively, aggregator $\mathrm{A}_{j}$ knows if it has aggregated a selected state from disseminator $D_{i}$ by checking aggregation membership $M_{A_{j}}\left(D_{i}\right)$. Moreover, $A_{j}$ knows if the specific selected state $S_{i}^{\prime}$ has been aggregated earlier by checking aggregation membership $M_{\mathrm{A}_{j}}\left(\mathrm{~S}_{i}^{\prime}\right)$. Therefore, both (i) duplicate and (ii) outdated selected states can be detected between an aggregator and a disseminator and the consistency of an aggregation session is satisfied.

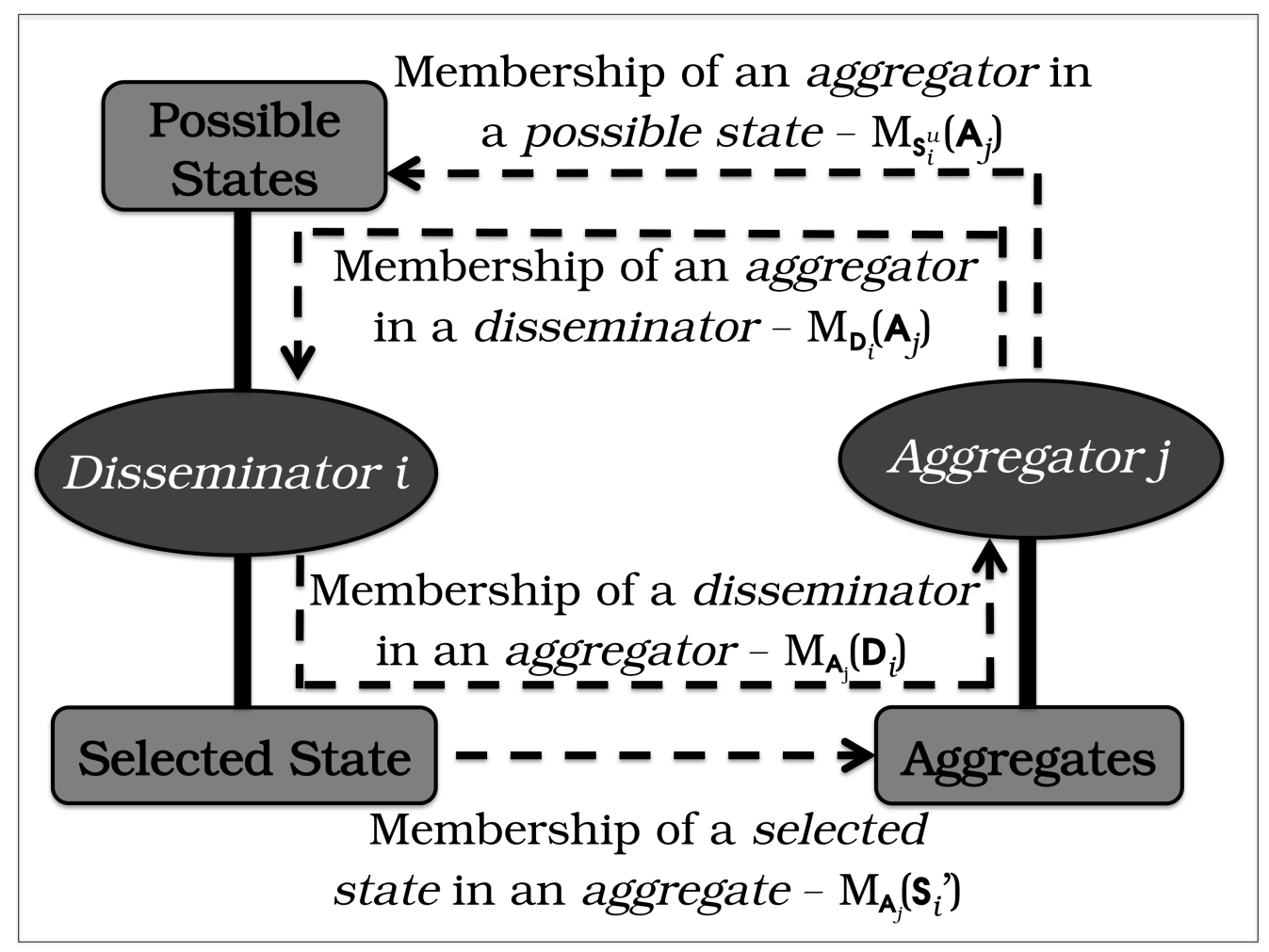

Figure 5.2: The aggregation memberships of DIAS.

Explicit storage of aggregation memberships in the agents of the structuring level is not a scalable and decentralized solution. Furthermore, in practice, aggregation memberships represent two mutual conditions resulting in information redundancy: Both aggregators and disseminators store membership information about their in-between aggregation. Section 5.7 shows how this redundancy is exploited in an efficient model realization of aggregation memberships based on bloom filters. 


\subsubsection{Classification}

Classification performed in the structuring level is based on an aggregation pool containing three aggregation views. These views are queues of a limited size in which aggregators are classified. Three aggregation views are defined in the aggregation pool: (i) exploited, (ii) unexploited and (iii) outdated. The exploited aggregators of a disseminator $\mathrm{D}_{i}$ are the ones that have aggregated its earliest selected state $s_{i}^{\prime}$. The unexploited aggregators of a disseminator $\mathrm{D}_{i}$ are the ones with which a consistent aggregation session has not been established and therefore, $D_{i}$ has not provided any of its selected states to these aggregators. Finally, the outdated aggregators of a disseminator $\mathrm{D}_{i}$ are the ones that have aggregated a selected state of this disseminator earlier but since then this selected state has changed. Aggregation views are used as a buffer and have a limited size to allow scalability and decentralization. Indicating size examples are examined in Section 5.8.4 and 5.8.5.

Figure 5.3 illustrates the classification of an aggregator $A_{j}$ in the aggregation pool based on the aggregation memberships $M_{D_{i}}\left(\mathrm{~A}_{j}\right)$ and $M_{\mathrm{S}_{i}^{\prime}}\left(\mathrm{A}_{j}\right)$ of a disseminator $\mathrm{D}_{i}$. When $A_{j}$ is received by the discovery level, the structuring level executes a membership query $M_{D_{i}}\left(\mathrm{~A}_{j}\right)$ that indicates if a consistent aggregation session has been performed between $\mathrm{A}_{j}$ and $D_{i}$. If membership is negative, aggregator $A_{j}$ is classified as unexploited. Otherwise, if membership is positive, the next membership query $M_{S_{i}^{\prime}}\left(A_{j}\right)$ is performed to indicate if aggregator $A_{j}$ has computed in its aggregates the most recent selected state $S_{i}^{\prime}$. If this membership is positive, aggregator $A_{j}$ is exploited (duplicate aggregation value), otherwise, aggregator $A_{j}$ has computed an earlier selected state of $D_{i}$ and therefore $A_{j}$ is classified as outdated.

If the selected state of disseminator $D_{i}$ changes, the aggregation pool requires rearrangement. Aggregators contained in the exploited view before the change of the selected state move to the outdated view. In contrast, aggregators contained in the outdated view before the change of the selected state are queried again $\left(M_{S_{i}^{\prime}}\left(\mathrm{A}_{j}\right)\right)$ and are classified as outdated or exploited according to Figure 5.3. As a result of this querying, the aggregation pool remains consistent and adapts instantly after a change of the selected state.

Note that the classification of aggregator samples in the aggregation pool realizes the structure subtask of Algorithm 3.10 in the ASMA architecture.

\subsubsection{Adaptation strategies}

A consistent aggregation session is established with either an unexploited or an outdated aggregator. The choice of the one over the other is related to aggregation dynamics, for example, speed of changes of the selected states and participant peers in the network. Priority can be given to aggregation sessions established with unexploited or outdated aggregators as defined by the structuring criteria received from the coordination level. These two options are the two adaptation strategies of DIAS and are referred to as EXPLOITATION and UPDATE respectively.

EXPLOITATION is a more relevant adaptation strategy if selected states do no change often and the aggregates still converge to their actual values, for example, at the beginning of aggregation or during network scaling with new peers. In contrast, UPDATE is more relevant for steady size of networks and when aggregates have converged to the actual values. Changes of the selected states after convergence require adaptations of 


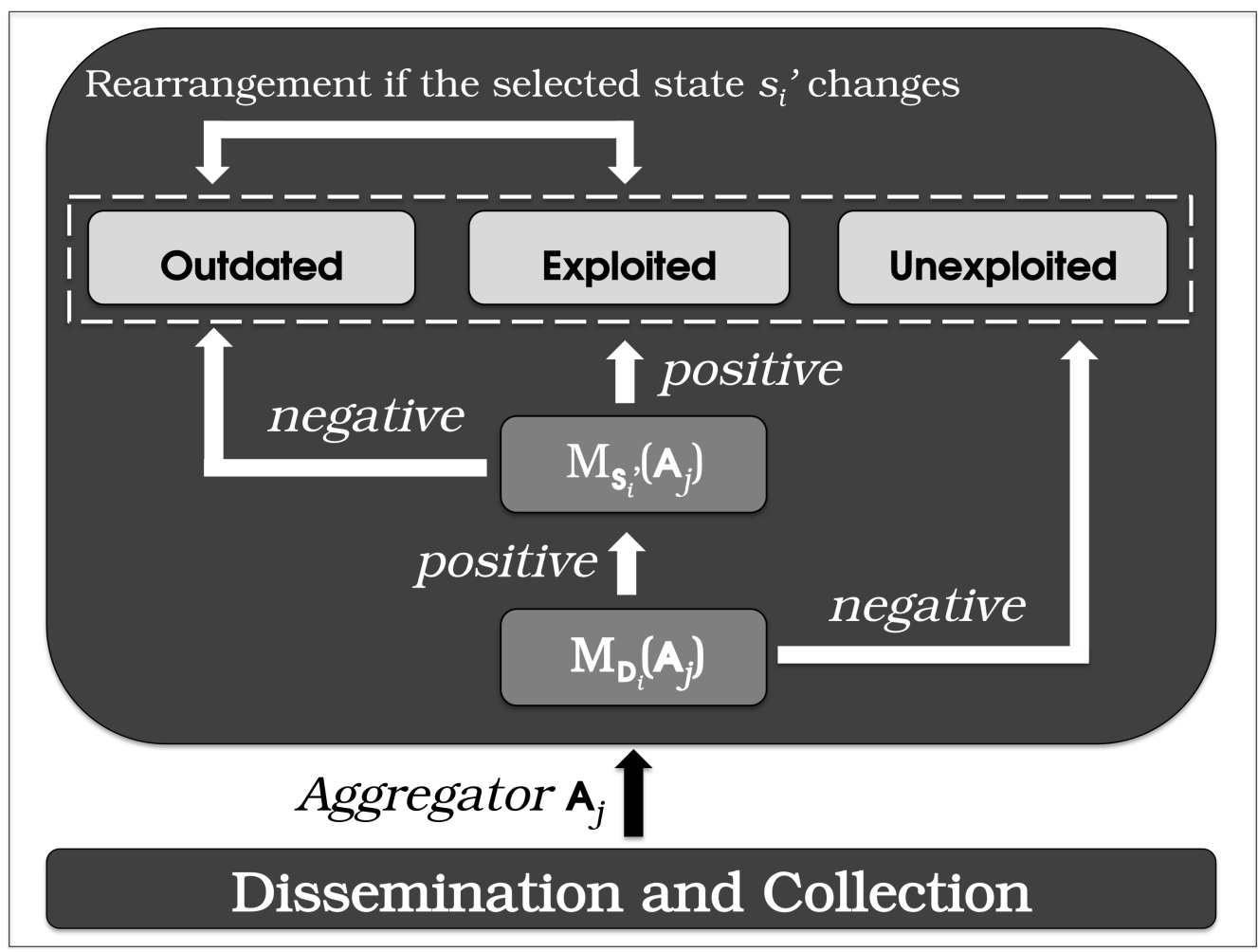

Figure 5.3: Classification of aggregators in the aggregation pool.

aggregates.

Selection of aggregators from the aggregation pool is conditional to the availability of aggregators in the class of preference for each adaptation strategy. This means that if ExPLOITATION is adopted but the unexploited view of the aggregation pool is empty, then outdated aggregators are selected corresponding to the selections of the UPDATE adaptation strategy. The same holds if the UPDATE adaptation strategy is adopted and the view of outdated aggregators is empty: unexploited aggregators are selected. To this extent, the adaptation strategies of DIAS can be considered to be dynamic.

The adaptation strategies of DIAS realize the selectToProvide subtask of Algorithm 3.9 in the ASMA architecture. The returned structured samples contain the selected aggregators and their class that is unexploited or outdated. Adoption of an adaptation strategy, realized by the adopt subtask of Algorithm 3.9 in the ASMA architecture, can be static, e.g., a system parameter contained in the classification criteria, or dynamic during system runtime. For example, the adopted adaptation strategy may change based on some monitored parameters or based on a time period that the aggregates do not change significantly.

\subsubsection{Aggregation session}

An aggregation session requires remote interactions between the aggregators and the disseminators of two peers $i$ and $j$ to guarantee its consistency. Figure 5.4 illustrates 
the remote interactions during a bidirectional aggregation session between a peer $i$ and $j$. A unidirectional aggregation session is established with horizontal interactions of ASMA. Two messages are exchanged as outgoing criteria and outgoing samples. The arrows (1) and (2) of Figure 5.4 correspond to these two messages. A bidirectional aggregation session includes a set of outgoing criteria within the message of the outgoing samples (arrow (2) of Figure 5.4) and completes with a third message of outgoing samples depicted by arrow (3) of Figure 5.4 .

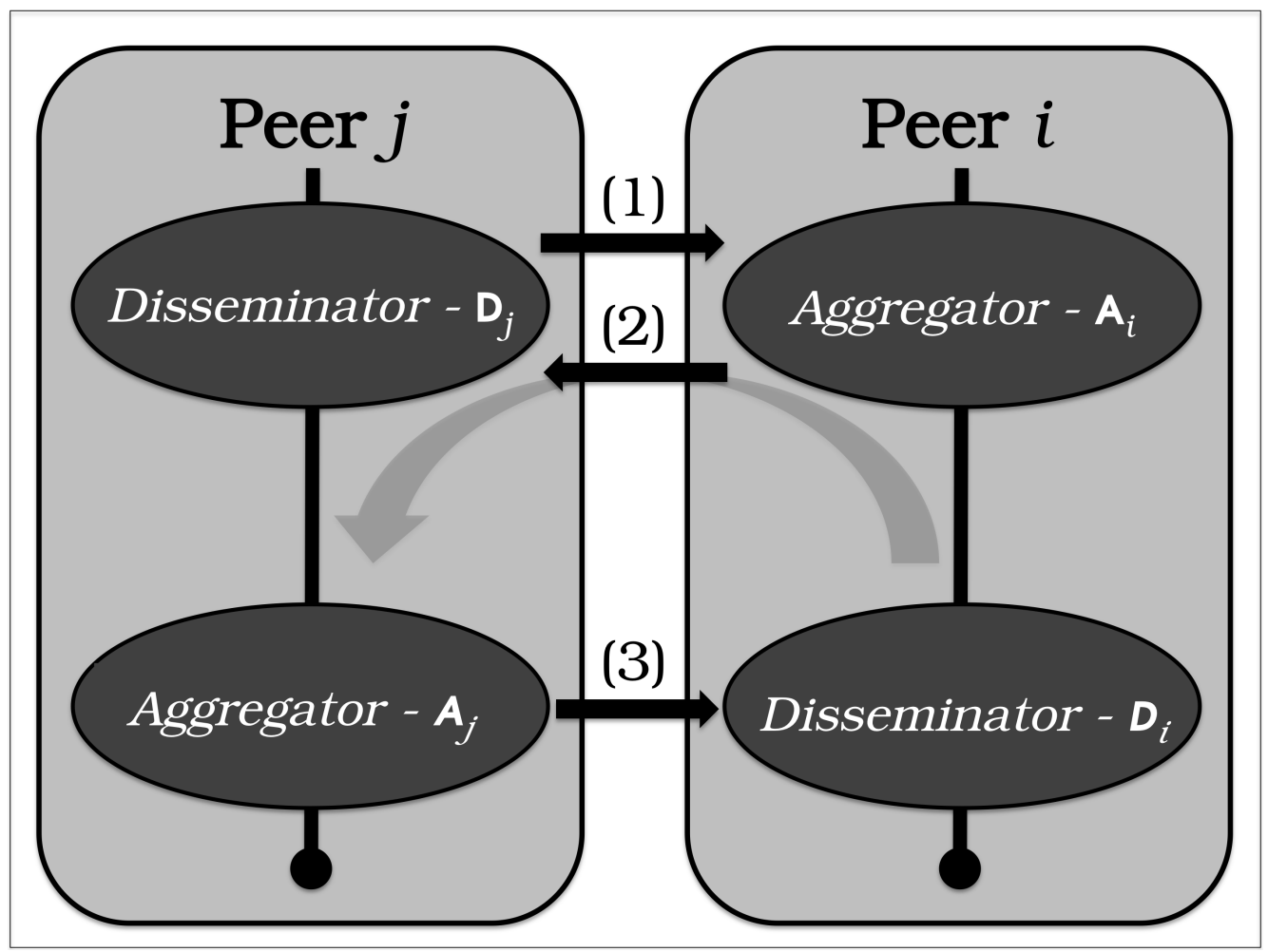

Figure 5.4: The remote interactions of a bidirectional aggregation session.

A set of incoming criteria initiates a unidirectional or bidirectional aggregation session and contains the following information illustrated for arrow (1) of Figure 5.4:

- Flag: This denotes a unidirectional 'uni' or bidirectional 'bi' aggregation session.

- Class: This denotes if the aggregator $\mathrm{A}_{i}$, receiving these criteria, is classified by a disseminator $\mathrm{D}_{j}$ as unexploited or outdated.

- $\mathrm{D}_{j}$ : This is the identifier of the disseminator $\mathrm{D}_{j}$ that has performed the classification of the aggregator $\mathrm{A}_{i}$.

- $\mathrm{S}_{j}^{\prime}$ : This is the selected state identifier of the disseminator $\mathrm{D}_{j}$.

- $\hat{S}_{j}$ : This is the earlier selected state identifier of the disseminator $D_{j}$ that the aggregator $\mathrm{A}_{i}$ has aggregated. 
A set of incoming samples completes a unidirectional or bidirectional aggregation session and contains the following information illustrated for arrow (2) of Figure 5.4:

- Flag: This denotes a unidirectional 'uni' or bidirectional 'bi' aggregation session. A third flag, the 'uni-bi', denotes the upgrade of a unidirectional aggregation session to a bidirectional one by including in the incoming samples a set of incoming criteria flagged as 'bi'.

- Class: This denotes if the aggregator $\mathrm{A}_{j}$, sending these samples, is classified by a disseminator $\mathrm{D}_{i}$ as unexploited or outdated.

- $\mathrm{A}_{j}$ : This is the identifier of the aggregator $\mathrm{A}_{j}$.

- Incoming criteria: These criteria are optional and integrated in the message of incoming samples. They upgrade the unidirectional aggregation session to a bidirectional one in the interaction depicted by the arrow (2) of Figure 5.4.

Algorithm 5.1 and 5.2 illustrate the interactions of an aggregation session. Note that these interactions refer to the optional parts of the adapt and consume tasks in Algorithm 3.9 and 3.10 respectively. An aggregation session is initiated by the select ToProvide subtask. Before a selected aggregator $\mathrm{A}_{i}$ is provided to the coordination level, a set of outgoing criteria is sent to $A_{i}$ to make its aggregation memberships consistent to the memberships of disseminator $D_{j}$. Lines 1-7 of Algorithm 5.1 illustrate the update of the aggregation memberships by aggregator $A_{i}$. Update of the memberships is performed according the classification outcome of $\mathrm{A}_{i}$ by disseminator $\mathrm{D}_{j}$. Aggregator $\mathrm{A}_{i}$ adds the memberships $M_{\mathrm{A}_{i}}\left(\mathrm{D}_{j}\right)$ and $M_{\mathrm{A}_{i}}\left(\mathrm{~S}_{j}^{\prime}\right)$ if it is classified as unexploited by $\mathrm{D}_{j}$ and additionally removes membership $M_{\mathrm{A}_{i}}\left(\hat{\mathrm{S}}_{j}\right)$ if it is classified as outdated. The possibility of performing a bidirectional aggregation session is checked in lines 9-18 of Algorithm 5.1 using the classification approach of Figure 5.3.

Similarly, the receipt of incoming samples by disseminator $D_{i}$ triggers the update of its aggregation memberships as shown in lines 1-7 of Algorithm 5.2. Disseminator $D_{i}$ adds the memberships $M_{\mathrm{D}_{i}}\left(\mathrm{~A}_{j}\right)$ and $M_{\mathrm{S}_{i}^{\prime}}\left(\mathrm{A}_{j}\right)$ if aggregator $\mathrm{A}_{j}$ is unexploited and additionally removes the membership $M_{\hat{S}_{i}}\left(A_{j}\right)$ if $A_{j}$ is outdated. This completes a unidirectional aggregation session. If a bidirectional aggregation session is performed, the adapt task is executed with the incoming criteria as an input (line 9 of Algorithm 5.2).

Note that the incoming criteria for a bidirectional aggregation session are integrated in the message of the incoming samples. This provides one message fewer for a bidirectional session to complete. This communication reduction may have a significant impact at the beginning of aggregation during which the majority of the aggregators are classified as unexploited and therefore the aggregations sessions are mostly bidirectional. The experimental findings of Section 5.8 confirm this.

\subsection{Computation of Aggregates}

The coordination level is responsible for the computation of aggregates. An aggregate is continuously computed based on an aggregation function provided by the aggregation criteria. The parameterize subtask of Algorithm 3.13 provides the aggregation function. Aggregates are updated by sending the value of the selected state to aggregators provided by the structuring level and classified as unexploited. If the provided 
$\overline{\text { Algorithm 5.1 The operations of an aggregation session embedded in the adapt task of the }}$ structuring level in DIAS.

Require: incoming criteria: flag, class, $\mathrm{D}_{j}, \mathrm{~S}_{j}^{\prime}, \hat{\mathrm{S}}_{j}$

1: add $M_{\mathrm{A}_{i}}\left(\mathrm{~S}_{j}^{\prime}\right)$

2: if class=unexploited then

3: $\quad$ add $M_{\mathrm{A}_{i}}\left(\mathrm{D}_{j}\right)$

4: end if

5: if class=outdated then

6: $\quad$ remove $M_{\mathrm{A}_{i}}\left(\hat{\mathrm{S}}_{j}\right)$

7: end if

8: if flag='uni' then

9: $\quad$ if $M_{D_{i}}\left(\mathrm{~A}_{j}\right)$ : negative then

10: $\quad$ outgoing criteria=get Criteria ('bi', unexploited, $\mathrm{D}_{i}, \mathrm{~S}_{i}^{\prime}, \hat{\mathrm{S}}_{i}$ )

11: outgoing samples=get Samples ('uni-bi', class, $A_{i}$, outgoing criteria )

12: $\quad$ else if $M_{D_{i}}\left(\mathrm{~A}_{j}\right):$ positive and $M_{\mathrm{S}_{i}^{\prime}}\left(\mathrm{A}_{j}\right):$ negative then

13: $\quad$ outgoing criteria=get Criteria ('bi', outdated, $\mathrm{D}_{i}, \mathrm{~S}_{i}^{\prime}, \hat{\mathrm{S}}_{i}$ )

14: outgoing samples=get Samples ('uni-bi', class, $A_{i}$, outgoing criteria )

15: $\quad$ else $/ / M_{\mathrm{D}_{i}}\left(\mathrm{~A}_{j}\right)$ : positive and $M_{S_{i}^{\prime}}\left(\mathrm{A}_{j}\right)$ : positive

16: outgoing samples=get Samples ( 'uni', class, $A_{i}$ )

17: end if

18: return outgoing samples

19: else // flag='bi'

20: $\quad$ outgoing samples $=$ get Samples ( 'bi', class, $\left.A_{i}\right)$

21: return outgoing samples

22: end if

Ensure: outgoing samples

$\overline{\text { Algorithm 5.2 The operations of an aggregation session embedded in the consume task of the }}$ structuring level in DIAS.

Require: incoming samples: flag, class, $\mathrm{A}_{j}$, incoming criteria

1: add $M_{S_{i}^{\prime}}\left(\mathrm{A}_{j}\right)$

2: if class=unexploited then

3: $\quad$ add $M_{\mathrm{D}_{i}}\left(\mathrm{~A}_{j}\right)$

4: end if

5: if class=outdated then

6: $\quad$ remove $M_{\hat{\mathrm{S}}_{i}}\left(\mathrm{~A}_{j}\right)$

7: end if

8: if flag='uni-bi' then

9: adapt (incoming criteria)

10: end if

aggregators are classified as outdated, the earlier selected state is sent as well. The initializecoordination subtask initiates this communication, the finalizeCoordination subtasks completes it and the organize subtask realizes the computation of the aggregation functions as defined in Algorithm 3.13 and 3.14 of the ASMA architecture. 
The coordination level forms an overlay network between aggregators and disseminators linked with overlay links that have two possible semantic values: unexploited or outdated but not exploited. Therefore, the aggregation functions computed exclude overlay links from the coordination level that result in duplicate aggregation values (exploited aggregators) as input. The aggregation memberships, the classification process, the selections of aggregators are all complexity that is hidden from the aggregation process of the coordination level. As explained in Section 5.5.3, the adaptation strategies tune the aggregation process in favor of (i) updating the aggregates with the most recent selected states (UPDATE) or (ii) discovering new selected states in the system (EXPLOITATION). The coordination level has to only provide the classification criteria that trigger this optimization and inform about changes in the selected state. Therefore, the coordination level remains agnostic about the details of the optimization.

Providing aggregates to the applications may be related to different requirements. For example, periodic delivery is an option. Another option is a minimum deviation threshold over a certain time period that may denote convergence to the actual aggregate values in the system. The aggregation criteria define these requirements.

\subsection{Realization based on Bloom Filters}

Explicit storage of aggregation memberships in every agent of the structuring level is not a scalable, efficient and decentralized solution. This section shows how aggregation memberships of DIAS can be a cost-effective and viable approach in large-scale decentralized environments using an implicit storage mechanism: bloom filters [Bloom, 1970, Brodera and Mitzenmacher, 2004].

A bloom filter is a probabilistic data structure for efficient membership storage and querying. A bloom filter is based on a number of $k$ hash functions that hash an element in a limited binary space of $2^{\mathrm{m}}$ size. More specifically, each hash function outputs a random index in this binary space.

A simple bloom filter supports insertions and membership queries. During an insertion, the bits that are indexed by the hash functions are set to 1. During membership queries, the membership of an element in the bloom filter is confirmed if all of the bits indexed by all of the hash functions are 1 .

Counting bloom filters additionally support removal of memberships as illustrated by Li et al. [2000]. This is achieved by representing the storage space with integers, instead of single bits, that act as counters. Insertions increment the counters indexed by the hash functions and removals decrement respectively. Data overflow by consecutive insertions is prevented by choosing an adequate size of $3-4$ bits for the integers. Therefore, a counting bloom filter is $3-4$ times larger than a simple one.

Each of the memberships illustrated in Figure 5.2 is stored in a bloom filter. More specifically, a disseminator $D_{i}$ has a simple bloom filter for storing $M_{D_{i}}\left(A_{j}\right)$ memberships and $v$ counting bloom filters, one for each possible state, for storing $M_{\mathrm{S}_{i}^{u}}\left(\mathrm{~A}_{j}\right)$ memberships. The counting bloom filters provide the flexibility to reflect the changes of the selected states. For example, in an aggregation session between a disseminator $D_{i}$ and an outdated aggregator $\mathrm{A}_{j}$, the membership $M_{\hat{S}_{i}}\left(\mathrm{~A}_{j}\right)$ is removed from the counting bloom filter of the earlier selected state $\hat{S}_{i}$ and the membership $M_{S_{i}^{\prime}}\left(A_{j}\right)$ is added in the counting bloom filter of the most recent selected state $S_{i}^{\prime}$. Complementarily, the aggregator $A_{j}$ has a simple bloom filter for storing the $M_{\mathrm{A}_{j}}\left(\mathrm{D}_{i}\right)$ memberships and a counting bloom 
filter for storing the $M_{\mathrm{A}_{j}}\left(\mathrm{~S}_{i}^{\prime}\right)$ memberships. Storing $M_{\mathrm{A}_{j}}\left(\mathrm{~S}_{i}^{\prime}\right)$ memberships in a counting bloom filter provides a consistent update of aggregates by replacing outdated selected states with the most recent ones.

The space saving achieved by bloom filters come at the cost of false positives. False positive membership indicates that a state or agent identifier is hashed in a bloom filter when it is actually not hashed. The probability of false positives depends on (i) the number of elements stored in the bloom filter, (ii) the number $k$ of hash functions used and (iii) the size $2^{\mathrm{m}}$ of the storage space. More specifically, the minimum number of bits in a simple bloom filter $x$ that hashes $n$ elements and results in a certain probability $P_{f p}(x)$ of false positives is computed as $2^{\mathrm{m}}=-n \frac{\ln P_{f p}(x)}{(\ln 2)^{2}}$ [Goel and Gupta, 2010]. False positives can cause inconsistent aggregation sessions (inaccurate aggregates) and additional communication overhead if they are not detected and eliminated.

The space savings computed for a bloom filter can be outlined as follows: Assume at least $128 n$ bits stored in conventional data structures such as an array. The $128 n$ bits are actually $n$ number of agent or state memberships represented by global unique identifiers of 128 bits. A hash table requires even a higher storage space due to the additional storage of indexes that enhance searching operations. In contrast, assume a bloom filter $x$ with a probability $P_{f p}(x)=0.01$ of false positives that stores the same number $n$ of memberships. The relation $2^{\mathrm{m}}=-n \frac{\ln P_{f p}(x)}{(\ln 2)^{2}}$ shows that, in this case, an array stores $128 / 9.6 \approx 13$ times the space of this bloom filter. For a bloom filter with $P_{f p}(x)=0.1$ and $P_{f p}(x)=0.001$, its space storage is approximately 56 and 9 times lower than the one of conventional data structures respectively.

Note that false negatives in counting bloom filters may only occur if an erroneous element removal is performed. This removal results in a biased and inconsistent probabilistic data structure. For example, if a removed element is not actually hashed in this bloom filter, then its removal changes bits indicating memberships of other elements that are actually hashed. Therefore, the data structure becomes inconsistent as Deke et al. [2010] shows. This chapter assumes that false negatives cannot be generated in principle if and only if removals are not performed from counting bloom filters. Otherwise, Section 5.7.3 illustrates how false negatives are prevented in DIAS if removals are performed.

\subsubsection{The mutual membership check}

DIAS deals with the problem of false positives in bloom filters by taking advantage of decentralized mutual membership checks between disseminators and aggregators. A mutual memberships check, denoted as ' $\mathrm{n}$ ' in this chapter, is the process of querying two memberships in a disseminator and an aggregator that are assumed to either be both present or not. For example, the aggregation memberships $M_{D_{i}}\left(A_{j}\right)$ and $M_{A_{j}}\left(D_{i}\right)$ between a disseminator $D_{i}$ and an aggregator $A_{j}$ are mutual. During an aggregation phase, a disseminator stores memberships of aggregator identifiers and, respectively, these aggregators store memberships of the respective disseminator identifiers resulting in mutual aggregation memberships. $M_{\mathrm{S}_{i}^{\prime}}\left(\mathrm{A}_{j}\right)$ and $M_{\mathrm{A}_{j}}\left(\mathrm{~S}_{i}^{\prime}\right)$ are also mutual memberships. Selected state $S_{i}^{\prime}$ of a disseminator $D_{i}$ is associated with the $M_{S_{i}^{\prime}}\left(A_{j}\right)$ membership of an aggregator $\mathrm{A}_{j}$. Respectively, aggregator $\mathrm{A}_{j}$ stores the $M_{\mathrm{A}_{j}}\left(\mathrm{~S}_{i}^{\prime}\right)$ membership of the selected state identifier $S_{i}^{\prime}$.

Mutual membership checks provide detection of false positives in the bloom filters of 
DIAS. Only if multiple false positives occur between $M_{\mathrm{D}_{i}}\left(\mathrm{~A}_{j}\right)-M_{\mathrm{A}_{j}}\left(\mathrm{D}_{i}\right)$ and $M_{\mathrm{S}_{i}^{\prime}}\left(\mathrm{A}_{j}\right)-M_{\mathrm{A}_{j}}\left(\mathrm{~S}_{i}^{\prime}\right)$ in a single aggregation session, then an inconsistent aggregation session may come as a result of these false positives.

Assume two arbitrary memberships $M_{x}(a)$ and $M_{y}(b)$ based on the unique identifiers of two members $a$ and $b$ in the groups $x$ and $y$ respectively. Assume also that these two memberships are mutual, meaning that they should be both positive or negative such as $M_{x}(a) \cap M_{y}(b)$ : positive $\mid M_{x}(a) \cap M_{y}(b)$ : negative. $M_{x}(a)$ and $M_{y}(b)$ are stored in two simple bloom filters with false positive probabilities $P_{f p}(x)$ and $P_{f p}(y)$ respectively. The possible outcomes of the mutual membership check are the following:

Check 1. if $M_{x}(a)$ : positive and $M_{y}(b)$ : positive then $M_{x}(a) \cap M_{y}(b):$ positive

$M_{x}(a)$ and $M_{y}(b)$ memberships are confirmed with a probability of $1-P_{f p}(x) P_{f p}(y)$. This confirmation is false if and only if both bloom filters generate a false positive that is the product $P_{f p}(x) P_{f p}(y)$ of their false positive probabilities.

Check 2. if $M_{x}(a)$ : positive and $M_{y}(b)$ : negative, or, $M_{x}(a)$ : negative and $M_{y}(b)$ : positive then $M_{x}(a) \cap M_{y}(b)$ : negative

$M_{x}(a)$ and $M_{y}(b)$ memberships are not confirmed with a probability of 1 . In this case, one of the bloom filters generates a false positive. False negatives cannot be generated by simple bloom filters.

Check 3. if $M_{x}(a)$ : negative and $M_{y}(b)$ : negative then $M_{x}(a) \cap M_{y}(b)$ : negative

$M_{x}(a)$ and $M_{y}(b)$ memberships are not confirmed with a probability of 1 as simple bloom filters cannot generate false negatives.

Mutual membership checks provide (i) a decrease in the probability that an inconsistent aggregation session occurs (Check 1) and (ii) detection of false positives (Check 2). This section introduces a consistency mechanism of aggregation sessions that supports accurate aggregates. This mechanism is based on two nested mutual membership checks between the bloom filters of an aggregator $A_{j}$ and a disseminator $D_{i}$. These mutual membership checks define four possible outcomes of an aggregation session:

- Exploitation: Aggregator $\mathrm{A}_{j}$ and disseminator $\mathrm{D}_{i}$ are involved for a first time in a consistent aggregation session as defined in Section 5.5. A selected state has not been aggregated before and the aggregates are updated with new information. The $M_{\mathrm{D}_{i}}\left(\mathrm{~A}_{j}\right), M_{\mathrm{A}_{j}}\left(\mathrm{D}_{i}\right), M_{\mathrm{S}_{i}^{\prime}}\left(\mathrm{A}_{j}\right)$ and $M_{\mathrm{A}_{j}}\left(\mathrm{~S}_{i}^{\prime}\right)$ memberships are added in the respective bloom filters.

- Update: Aggregator $\mathrm{A}_{j}$ and disseminator $\mathrm{D}_{i}$ have been involved before in a consistent aggregation session, however, this time the selected state has changed. The aggregator $\mathrm{A}_{j}$ updates its aggregates with the new selected state. The $M_{\hat{S}_{i}}\left(\mathrm{~A}_{j}\right)$ membership is replaced by the $M_{S_{i}^{\prime}}\left(A_{j}\right)$ membership and the $M_{A_{j}}\left(\hat{S}_{i}\right)$ membership is replaced by the $M_{\mathrm{A}_{j}}\left(\mathrm{~S}_{i}^{\prime}\right)$ membership.

- Duplicate: Aggregator $\mathrm{A}_{j}$ and disseminator $\mathrm{D}_{i}$ have been involved before in an aggregation session with the same selected state. Aggregation is not performed and no actions are applied to their bloom filters. 
- Inconsistency: Aggregator $\mathrm{A}_{j}$ and the disseminator $\mathrm{D}_{i}$ are involved for a first time in a consistent aggregation session but the mutual membership check cannot confirm this. Alternatively, aggregator $A_{j}$ and disseminator $D_{i}$ have been involved before in an aggregation session with a different selected state. However, the consistency check cannot identify the outdated selected state to replace. These uncertainties are treated as an inconsistency and are a result of multiple false positives in the bloom filters of DIAS.

The two nested mutual membership checks illustrated in Section 5.7.2 and 5.7.3 show how an aggregation session reaches each of the above possible outcomes. The nested membership checks can be performed in both the disseminator and aggregator if the results of the memberships are exchanged in the messages defined in Section 5.5.4. Therefore, there is no additional communication required for this purpose. Note that the results of the memberships exchanged concern the boolean results of the bloom filter queries. There is no need for agents to exchange their bloom filters that are always stored locally.

\subsubsection{First level check}

This mutual membership check identifies if a consistent aggregation session has not been performed between an aggregator $A_{j}$ and a disseminator $D_{i}$. Disseminator $D_{i}$ queries the $M_{D_{i}}\left(\mathrm{~A}_{j}\right)$ membership of the $\mathrm{A}_{j}$ identifier in its bloom filter. Complementarily, aggregator $\mathrm{A}_{j}$ queries $M_{\mathrm{A}_{j}}\left(\mathrm{D}_{i}\right)$ membership. The $M_{\mathrm{D}_{i}}\left(\mathrm{~A}_{j}\right)$ and $M_{\mathrm{A}_{j}}\left(\mathrm{D}_{i}\right)$ memberships are mutual as they are either both added in the bloom filters or not. Therefore, a mutual membership check provides the following benefits at the first level of the nested mutual membership check: (i) A decrease in the probability of an inconsistent aggregation session that requires two false positives generated by the two bloom filters. (ii) Detection of a false positive in either the $M_{D_{i}}\left(A_{j}\right)$ or $M_{A_{j}}\left(D_{i}\right)$ membership. Recall that false negatives do not occur in simple bloom filters. Algorithm 5.3 illustrates the first level of the nested mutual membership check.

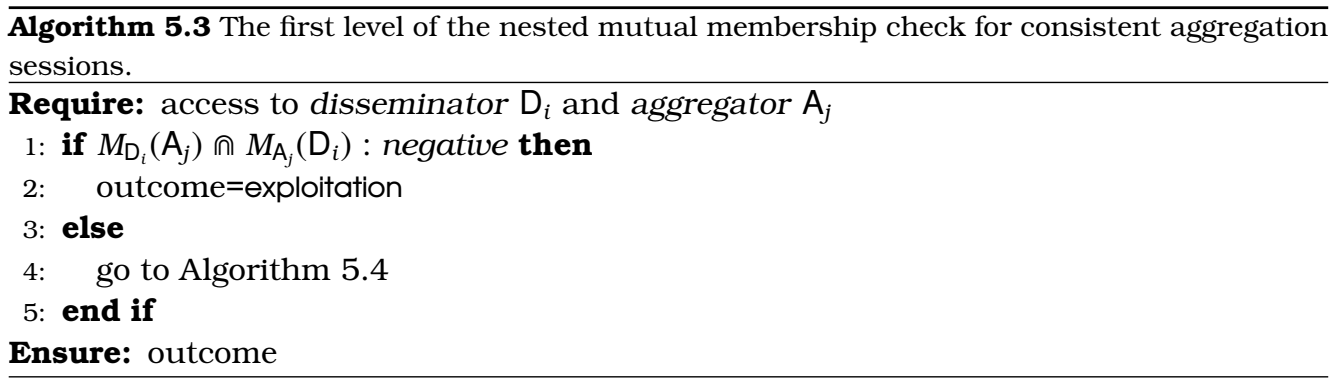

This mutual membership check detects an exploitation outcome between a disseminator $D_{i}$ and an aggregator $A_{j}$ if and only if $M_{D_{i}}\left(A_{j}\right) \cap M_{A_{j}}\left(D_{i}\right)$ : negative holds. This outcome is generated if at least one of the $M_{\mathrm{D}_{i}}\left(\mathrm{~A}_{j}\right)$ and $M_{\mathrm{A}_{j}}\left(\mathrm{D}_{i}\right)$ memberships, in case of a single false positive, or both memberships, in case of no false positives, cannot be confirmed in the respective bloom filters. On this first level of the nested memberships check, the exploitation outcome is reached with an absolute certainty as false negatives cannot be generated at this stage. However, two simultaneous false positives 
in the $M_{D_{i}}\left(\mathrm{~A}_{j}\right)$ and $M_{\mathrm{A}_{j}}\left(\mathrm{D}_{i}\right)$ memberships are possible. Therefore, further examination is required on a second level of a mutual membership check to detect multiple false positives and lower the uncertainties of the outcomes in an aggregation session.

\subsubsection{Second level check}

The second level of the mutual membership check detects if there is an outdated selected state $\hat{S}_{i}$ aggregated from a disseminator $D_{i}$ that differs from its new selected state $S_{i}^{\prime}$. The detection is performed by querying every $M_{S_{i}^{u}}\left(A_{j}\right)$ bloom filter membership of the respective possible state $S_{i}^{u} \in\left\{S_{i}^{0}, \ldots, S_{i}^{v-1}\right\} . \quad M_{A_{j}}\left(S_{i}^{u}\right)$ membership is also queried for every possible state $S_{i}^{u}$. The number $o$ of positive mutual memberships $M_{S_{i}^{u}}\left(A_{j}\right)$ ก $M_{\mathrm{A}_{j}}\left(\mathrm{~S}_{i}^{u}\right)$ define the outcome of an aggregation session. This mutual membership check is illustrated in Algorithm 5.4.

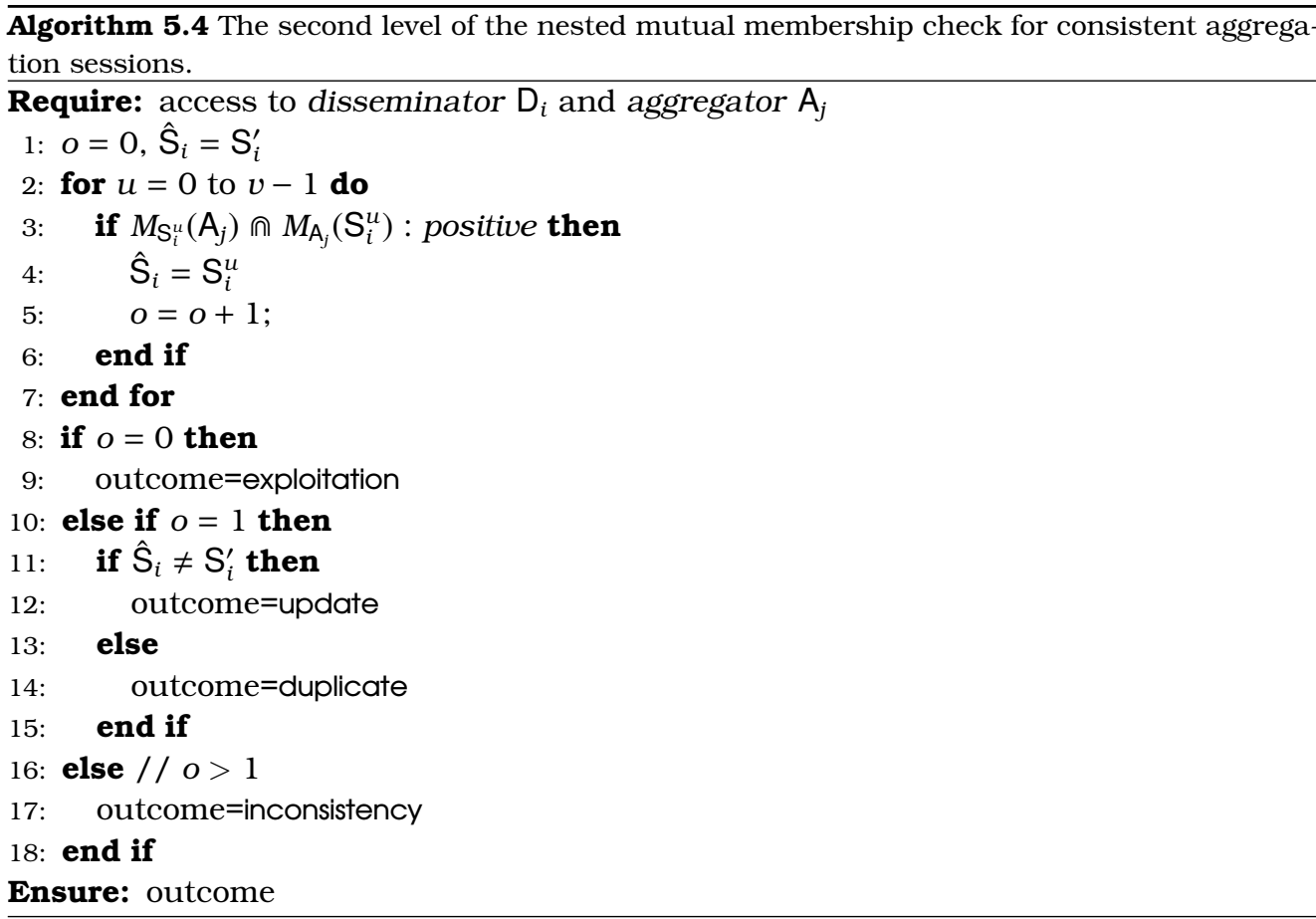

If there are no positive mutual memberships detected $(o=0$ in line 8 and 9 of Algorithm 5.4), there is no positive $M_{\mathrm{A}_{j}}\left(\mathrm{~S}_{i}^{u}\right)$ membership in the bloom filter of $\mathrm{A}_{j}$ (no selected state aggregated before from $\left.D_{i}\right)$ and/or there is no positive $M_{S_{i}^{u}}\left(A_{j}\right)$ membership in any bloom filter of the possible states. This condition conflicts with the positive result of the mutual membership check $M_{\mathrm{D}_{i}}\left(\mathrm{~A}_{j}\right) \cap M_{\mathrm{A}_{j}}\left(\mathrm{D}_{i}\right)$ in the first level. False negatives cannot occur in those bloom filters and therefore, both $M_{D_{i}}\left(A_{j}\right)$ and $M_{A_{j}}\left(D_{i}\right)$ memberships are false positives. The outcome in this case is an exploitation.

If there is one positive mutual membership detected $(o=1$ in lines 10-15 of Algorithm 5.4), the system can derive the outdated selected state $\hat{S}_{i}$. The outcome is either a duplicate, if the outdated selected state $\hat{S}_{i}$ is the same with the new selected state $S_{i}^{\prime}$, 
or an update in the opposite case. The uncertainty of this outcome is minimized by the nested mutual membership checks.

Finally, if more than one positive mutual membership is detected $(o>1$ in lines 16-18 of Algorithm 5.4), multiple false positives occur that cannot be identified. These false positives concern the $M_{S_{i}^{u}}\left(\mathrm{~A}_{j}\right)$ and $M_{\mathrm{A}_{j}}\left(\mathrm{~S}_{i}^{u}\right)$ memberships, or the $M_{\mathrm{D}_{i}}\left(\mathrm{~A}_{j}\right)$ and $M_{\mathrm{A}_{j}}\left(\mathrm{D}_{i}\right)$ memberships in the first level of the nested mutual membership check. The outcome is an inconsistency and therefore, any aggregation at this point may result in inaccuracies of the aggregates.

Inconsistencies can be handled in various ways. The 'safer' approach followed by DIAS is to ignore these aggregation sessions and not perform any aggregation that may result in inaccurate computations of the aggregates. However, not only the aggregates can be influenced in this case. Recall from the beginning of this section that removal of a membership from a counting bloom filter that is actually not present introduces false negatives according to Deke et al. [2010]. Therefore, the following aggregation sessions are prone to inaccuracies as the assumption of no false negatives does not hold anymore. By skipping inconsistent aggregation sessions, DIAS makes sure that the condition of no false negatives in bloom filters is not violated.

\subsection{Experimental Evaluation}

DIAS is implemented and evaluated in Protopeer [Galuba et al., 2009], a prototyping toolkit for distributed systems illustrated in Section 2.4. Table 5.1 summarizes the selected experimental settings. Note that multiple values for a single parameter denote the tested variations of this parameter in some of the illustrated experiments. The values depicted with bold are the default ones.

A network of $n=1500$ peers is simulated running DIAS for $t$ (DIAS) $=800$ epochs. The agents of each peer act both as aggregators and disseminators. Each epoch lasts for $T$ (DIAS) $=1000 \mathrm{~ms}$. Protopeer initially bootstraps peers in a ring topology. The bootstrapping period is $t^{\prime}(\mathrm{DIAS})=6$ epochs and the size of the ring view is $\mid \mathbf{v}($ ring $) \mid=5$ for each peer.

A simulated application of dynamically changing states is bootstrapped in $t^{\prime}$ (application $)=15$ epochs. Each application instance in the peers of the network generates $v=5$ numerical possible states in a single aggregation phase. The case of $v=2$ is also evaluated to show the effect of a lower number of possible states. The numerical values of the possible states are selected randomly from the range $[0,1)$ of input values defined by five different beta distributions, one for each possible state. The control of the skew in beta distribution, by changing the alpha and beta parameters, provides different random input domains for each possible state. Figure B.1 of Appendix B illustrates these beta distributions. The selected state changes cyclically as $s_{i}^{\prime}=s_{i}^{0}, s_{i}^{1}, \ldots, s_{i}^{v-1}, s_{i}^{0}$, etc. Two factors trigger a change of a selected state: (i) time and (ii) the parameter itself that the possible states represent. These two factors are modeled based on two probabilities: (i) the probability $P_{c}$ (time) of changing a selected state every period $T$ (application) and (ii) the probability $P_{c}$ (parameter) of change in a specific type of application parameter. The probability $P_{c}\left(s_{i}^{\prime}\right)$ of a peer $i$ to change its selected state is calculated as $P_{c}\left(s_{i}^{\prime}\right)=P_{c}($ time $) P_{c}$ (parameter) assuming that the two probabilities $P_{c}$ (time) and $P_{c}$ (parameter) are independent. Note that this simplified way of modeling changes in the selected states serves the simulation purposes of the model. By choosing 
Table 5.1: The experimental settings for the evaluation of DIAS. The bold values are the default ones in the performed experiments.

\begin{tabular}{|c|c|c|}
\hline & Parameter & Value \\
\hline \multirow{5}{*}{ DIAS } & $n$ & 1500 \\
\hline & $t$ (DIAS) & 800 \\
\hline & $T$ (DIAS) & 1000 \\
\hline & $t^{\prime}(\mathrm{DIAS})$ & 6 \\
\hline & $\mid \mathbf{v}$ (ring) $\mid$ & 5 \\
\hline \multirow{13}{*}{ Application } & $t^{\prime}$ (application) & 15 \\
\hline & $v$ & 2,5 \\
\hline & type of states & numerical \\
\hline & input domain of states & {$[0,1)$} \\
\hline & generation of possible states & beta distribution \\
\hline & distribution for $s_{i}^{0}$ & alpha $=5$, beta $=25$ \\
\hline & distribution for $s_{i}^{1}$ & alpha $=25$, beta $=5$ \\
\hline & distribution for $s_{i}^{2}$ & alpha $=10$, beta $=5$ \\
\hline & distribution for $s_{i}^{3}$ & alpha $=5$, beta $=10$ \\
\hline & distribution for $s_{i}^{4}$ & alpha $=5$, beta $=5$ \\
\hline & selection of a possible state & cyclical \\
\hline & T(application) & 10 (asynchronous), 200 (synchronous) \\
\hline & $P_{c}($ time $), P_{c}($ parameter $)$ & $(1.0,1.0),(0.4,0.7)$ \\
\hline \multirow{2}{*}{ Coordination level } & $T$ (coordination level) & 1000 \\
\hline & $f()$ & AVERAGE, SUMMATION, MAXIMUM \\
\hline \multirow{7}{*}{ Structuring level } & $T$ (structuring level) & 1000 \\
\hline & $z$ & $5, \mathbf{1 0}$ \\
\hline & $q$ & $30, \mathbf{4 5}$ \\
\hline & $e$ & $7, \mathbf{1 5}, 30$ \\
\hline & adaptation strategy adoption & static \\
\hline & hashing scheme & double hashing \\
\hline & $\mathrm{m}, \mathrm{k}$ & $(16,24),(14,24),(14,6)$ \\
\hline \multirow{5}{*}{ Discovery level } & $r$ & 50 \\
\hline & $T$ (discovery level) & 250, 1000 \\
\hline & view selection policy & swapper \\
\hline & view propagation policy & push-pull \\
\hline & peer selection policy & random \\
\hline
\end{tabular}

different values for $T$ (application), $P_{c}($ time $)$, and $P_{c}$ (parameter), a wide range of dynamic settings can be tested.

Two types of changes in the selected states are examined: synchronous and asynchronous. In synchronous changes, the selected states of all peers in the network change simultaneously. Synchronous changes are modeled as $P_{c}($ time $)=1$ and $P_{c}(p a-$ rameter $)=1$ for $T$ (application) $=200$ epochs. In contrast, asynchronous changes occur arbitrary over time. A dynamic setting of asynchronous changes is modeled as $P_{c}($ time $)=0.4$ and $P_{c}($ parameter $)=0.7$ for $T($ application $)=10$ epochs.

The execution period of the coordination level is synchronized with the one of the structuring level as $T($ coordination level $)=T($ structuring level $)=1000 \mathrm{~ms}$. The AvERAGE, SUMMATION and MAXIMUM aggregation functions ${ }^{6}$ are computed. The messages ex-

\footnotetext{
${ }^{6}$ Results are also available upon request for the following aggregation functions: SUMMATION SQUARE, MINIMUM,
} 
changed by the structuring level and coordination level are integrated. This minimizes the number of exchanged messages $\lambda$ (sessions) in DIAS. Therefore, the only messages exchanged by DIAS are at maximum the three ones of an aggregation session as illustrated in Section 5.5.4. The integrated messages additionally contain the actual states for the computation of the aggregation functions. The aggregates are provided to the application after every computation.

The structuring level is periodically executed at $T$ (structuring level) $=1000 \mathrm{~ms}$ during which $z=10$ bidirectional aggregation sessions are initiated at maximum. The convergence speed of DIAS is compared with $z=5$. The size of the aggregation pool is selected to $q=3 * 15=45$ with the unexploited, exploited and outdated views containing 15 aggregators at maximum. The alternative size of $q=3 * 10=30$ and the size of 25, 5 and 15 for the unexploited, exploited and outdated views respectively are also evaluated. The aggregation pool is filled by classifying $e=15$ random aggregator samples collected from the discovery level in each execution period. The impact of the number of collected samples is evaluated with a comparison to $e=7$ and $e=30$. Static adoptions of the EXPLOITATION and UPDATE adaptation strategies are evaluated.

Aggregation memberships are realized in the bloom filters of the XSiena BloomFilter ${ }^{7}$ library of Jerzak and Fetzer [2008]. The double hashing method of Dillinger and Manolios [2004] is used for collision resolution in the hashed elements of bloom filters. The implementation of double hashing is part of the XSiena BloomFilter library. The size $2^{\mathrm{m}}$ of the bloom filters and the number of hash functions $k$ are selected empirically using the testing tools of the BloomFilter library. The expected number of hashed elements during the performed experiments is equal to the network size $n$. This selection is performed manually during system parameterization or in an automated fashion. In the latter case, DIAS is initialized with a default size of bloom filters and computes the system size using the count aggregation function. This aggregate is able to indicate if the bloom filters have an adequate size.

Three schemes are adopted in DIAS: (i) $\mathrm{m}=16, \mathrm{k}=24$, (ii) $\mathrm{m}=14, \mathrm{k}=24$ and (iii) $\mathrm{m}=14, \mathrm{k}=6$. The first scheme, with $2^{16}=65536$ bits $=8.192 \mathrm{~KB}$, does not result in false positives during the performed library tests, whereas false positives appear in the other two schemes because of the fewer number of bits available for hashing: $2^{14}=16384$ bits $=2.048 \mathrm{~KB}$. The relation $2^{m}=-n \frac{\ln p}{(\ln 2)^{2}}$ verifies the probability of false positives for each scheme. For $n=1500$, the probability of false positives in the first scheme is $0.76 * 10^{9}$, whereas, for the other two schemes is 0.005 . The second scheme introduces higher randomness compared to the third one due to the higher number of hash functions. However, the second scheme causes a higher number of bit changes during insertions. This results in a higher number of potential collisions [Dillinger and Manolios, 2004] that cause a higher number of false positives.

The discovery level is realized by the peer sampling service [Jelasity et al., 2007]. The size of the random view is $r=50$ and the execution period is $T$ (discovery level) $=$ $T$ (DIAS) $/ 5=250 \mathrm{~ms}$. The execution period of $500 \mathrm{~ms}$ is also examined to study its influence on convergence speed. The values of the 'view selection', 'view propagation' and 'peer selection' policies are selected to maximize the randomness and dissemination

STANDARD DEVIATION and COUNT. The aggregation functions with the most representative and relevant findings are shown in this chapter, i.e., the duplicate-sensitive summation, the commonly used AVERAGE and the duplicate-insensitive MAXIMUM.

${ }^{7}$ Available at: http://wwwse.inf.tu-dresden.de/xsiena/bloom_filter (Last accessed: June 2011) 
speed of gossiping. More information about these parameters are provided by Jelasity et al. [2007].

\subsubsection{Evaluation outline}

The efficiency of DIAS is related to how close the values of the computed aggregates are to the actual ones. This closeness is quantified by two evaluation metrics: (i) accuracy $a$ and (ii) matching $\mu$. Accuracy $a$ is defined as $a=1-\varepsilon / \varepsilon_{\max }$ where $\varepsilon$ is the absolute error and $\varepsilon_{\max }$ is the maximum probable absolute error. The absolute error is the absolute difference of the actual aggregate from the computed aggregate. The maximum probable absolute error is the maximum possible absolute difference that the actual aggregate and the computed aggregate can have. Note that the convergence of accuracy is particularly interesting for the evaluation of DIAS as it outlines its speed and adaptivity in the computation of aggregates. Matching $\mu$ is based on the calculation of the correlation coefficient [Rodgers and Nicewander, 1988] and indicates the closeness of the distribution of the computed aggregates to the distribution of the actual aggregates. This metric is especially useful for the evaluation of DIAS under asynchronous changes in which aggregates are highly dynamic. The accuracy and matching are two possible performance metrics that quantify the quality of the DIAS overlay service.

The source data from which accuracy is computed are illustrated in Figure B.2, B.3, B.4 and B.5 of Appendix B. Both accuracy and matching are studied in line with the communication cost of the aggregation sessions in terms of the number of messages $\lambda$ (sessions) exchanged by agents. The communication cost of the discovery level is excluded from the illustrated results as it is constant and is discussed in Section 5.8.8. Finally, the results are interpreted based on the number of aggregation outcomes that the aggregation sessions result in.

Seven aspects of DIAS are examined in the performed experiments:

1. The adaptation strategies.

2. The bloom filter schemes.

3. The size of the aggregation pool.

4. The (relative) size of the unexploited, exploited and outdated views.

5. The number of random aggregator samples collected from the discovery level.

6. The number of aggregation sessions established periodically.

7. The adjustment of periodical executions in the three levels of DIAS.

The rest of this section illustrates the experimental results for each of these aspects.

\subsubsection{Adaptation strategies}

This section evaluates the efficiency of DIAS with and without adaptation strategies. For this reason, the bloom filter scheme of $m=16$ and $k=24$ is adopted that does not result in false positives according to the empirical tests. The case when DIAS does not employ adaptation strategies is referred to as the RANDOM strategy and concerns 
random samples directly received from the discovery level without a classification in the aggregation pool.

Figures 5.5a-5.5c illustrate the convergence of accuracy under synchronous changes. The EXPLOITATION and uPDATE adaptation strategies converge to the maximum accuracy $a=1$ and adapt the aggregates within 100 epochs. The matching $\mu$ achieved with these two adaptation strategies is $0.79,0.59$ and 0.90 for AVERAGE, SUMMATION and MAXIMUM respectively. The distribution of the exploitation and update outcomes depicted in Figure 5.6a and 5.6b explains the convergence of accuracy. These outcomes represent $z=10$ bidirectional aggregations sessions by $n=1500$ aggregators: $10 * 2 * 1500=30000$ exploitation and update outcomes. Note that under synchronous changes, EXPLOITATION and UPDATE have the same effect. The total number of aggregation sessions with an exploitation outcome are performed within the first $T$ (application) $=200$ epochs for both adaptation strategies. The next aggregation sessions result in update outcomes.

RANDOM also achieves a high accuracy according to Figures 5.5a-5.5c, with 0.71, 0.33 and 0.90 matching $\mu$ for each aggregate respectively. However, RANDOM has a slower convergence of 150 additional epochs compared to EXPLOITATION and UPDATE. This is because of the number of duplicate outcomes that reaches 28000 during convergence as depicted in Figure 5.6c. EXPLOITATION and UPDATE do not cause duplicate outcomes as the exploited aggregators are not selected from the aggregation pool.

Figure 5.5d-5.5f illustrate the convergence of accuracy $a$ under asynchronous changes. Although $P_{c}($ time $) P_{c}($ parameter $) n=0.4 * 0.7 * 1500=420$ selected states change on average every $T$ (application) $=10$ epochs, accuracy converges to the maximum for the adaptation strategies. Matching $\mu$ between the actual and computed AVERAGE for EXPLOITATION and UPDATE is 0.57 and 0.70 respectively. The RANDOM adaptation strategy is not influenced significantly and achieves a matching of $\mu=0.66$ for the AVERAGE aggregate. This is because RANDOM reaches exploitation and update outcomes during the converge period in contrast to EXPLOITATION that mostly reaches exploitation outcomes during the first 100 epochs (Figure 5.6d) and update outcomes in the next epochs (Figure 5.6e). Similarly with the case of synchronous changes, RANDom requires 150 additional epochs to converge compared to ExPLOITATION. A converged number of 10000 duplicate outcomes depicted in Figure 5.6f causes this delay. The matching $\mu$ achieved for the MAXIMUM aggregate is $0.67,0.55$ and 0.45 respectively for EXPLOITATION, UPDATE and RANDOM. Note that SUMMATION is more challenging to compute. ExPLOITATION provides the fastest convergence within the first 100 epochs. RANDOM converges in approximately 250 epochs. UPDATE does not converge before the 400th epoch as it does not prefer aggregators from the unexploited view and is influenced by the continuous changes of the selected states.

Figure 5.7 illustrates the messages $\lambda$ (sessions) sent during the aggregation sessions of DIAS. Under synchronous changes, the distribution of the communication cost during runtime depicted in Figure 5.7a corresponds to the exploitation and update outcomes in Figure 5.6a and 5.6b respectively. The ExPLOITATION and UPDATE adaptation strategies minimize the messages exchanged to 0 when the aggregates converge to their actual values. This is not the case for RANDOM that continuously exchanges $\lambda$ (sessions) $=$ $n z 3=1500 * 10 * 3=45000$ messages during runtime. These messages are generated by 1500 peers that periodically establish 10 bidirectional aggregation sessions with 3 messages exchanged in each session. This is the how the communication cost is estimated for larger networks or a different frequency of aggregation sessions. Under 


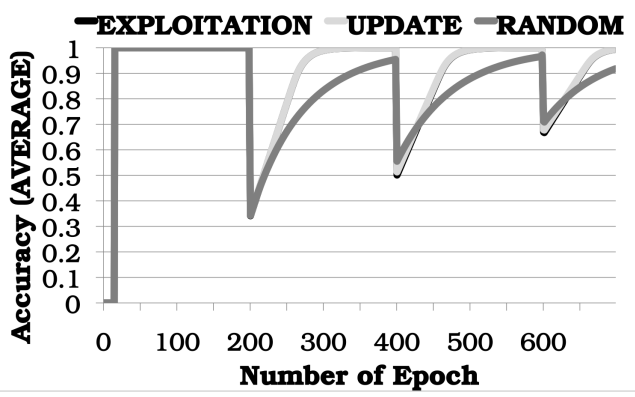

(a) Average under synchronous changes.

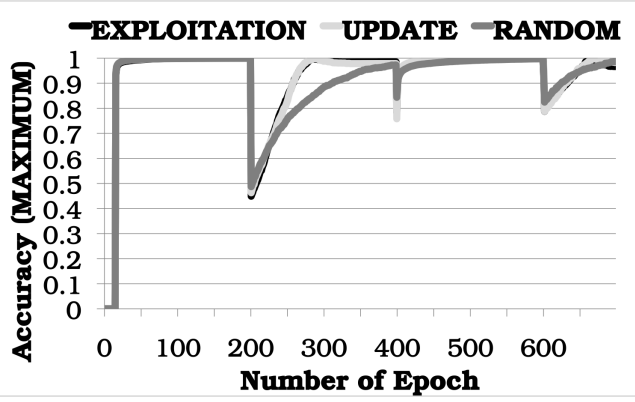

(c) Maхimum under synchronous changes.

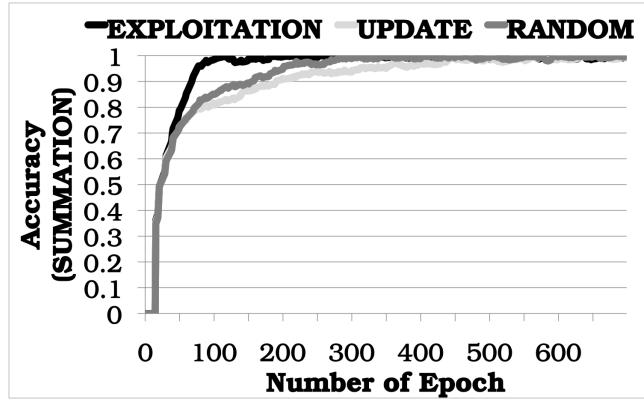

(e) Summation under asynchronous changes.

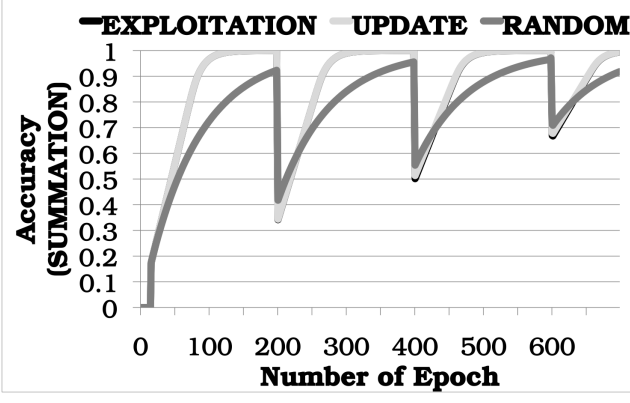

(b) Summation under synchronous changes.

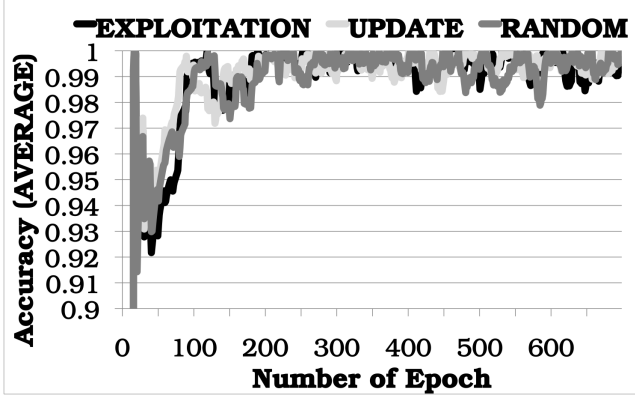

(d) Average under asynchronous changes.

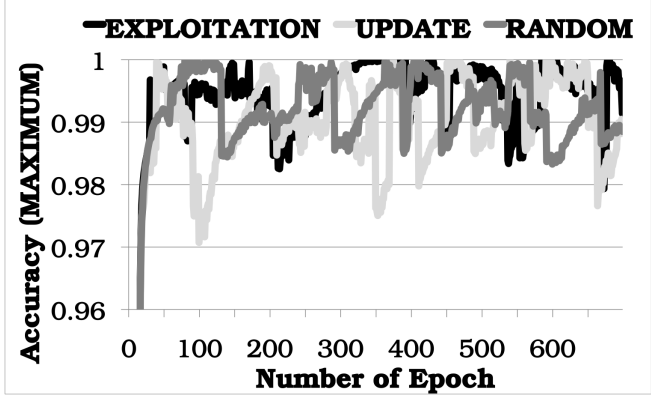

(f) MAXімuM under asynchronous changes.

Figure 5.5: Convergence of accuracy $a$ for the EXPLOITATION, UPDATE and RANDOM adaptation strategies.

asynchronous changes and during convergence in the first 100 epochs, ExPLOITATION and UPDATE exchange the maximum number of 38000-45000 messages that converges to 38000 in the next epochs during which update outcomes are mainly reached.

This communication cost becomes significantly lower if the peers of the network do no run both an aggregator and a disseminator agent. For example, if the network has 500 of its peers with an aggregator and the rest 1000 peers with a disseminator, the communication cost is computed in this case as $1000 * 10 * 2=20000$ messages that is significantly lower than the upper communication cost examined in this chapter. 


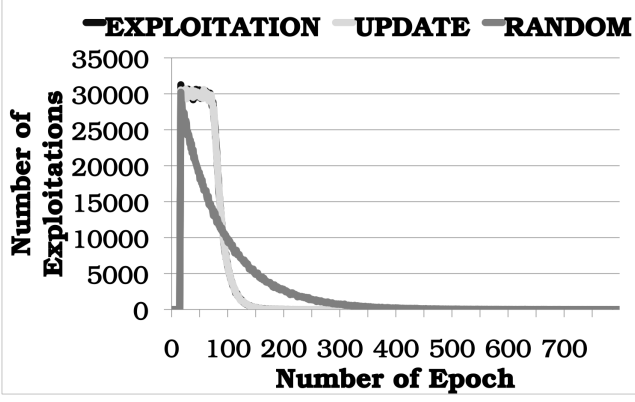

(a) Exploitation outcomes under synchronous changes.

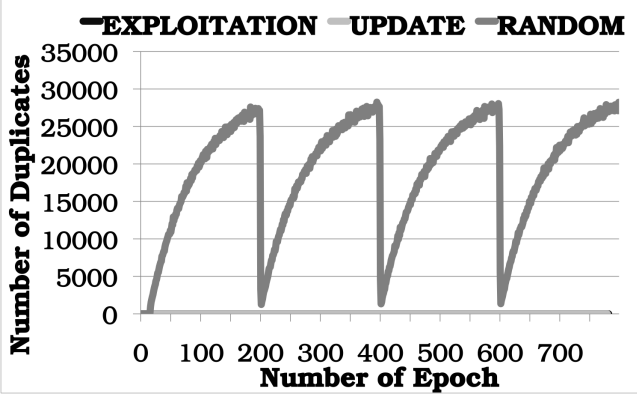

(c) Duplicate outcomes under synchronous changes.

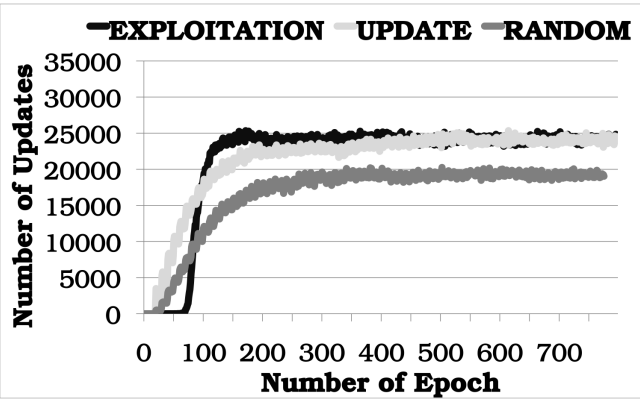

(e) Update outcomes under asynchronous changes.

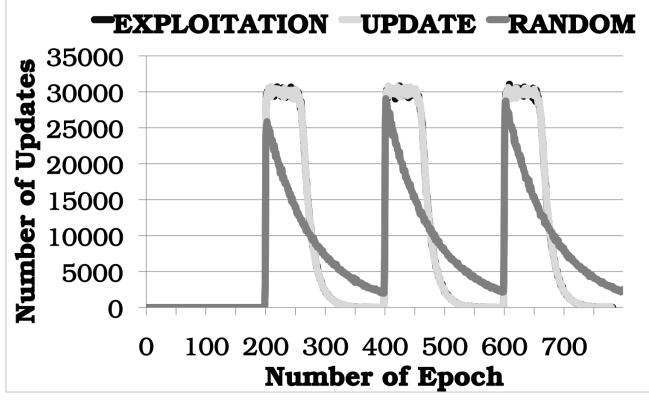

(b) Update outcomes under synchronous changes.

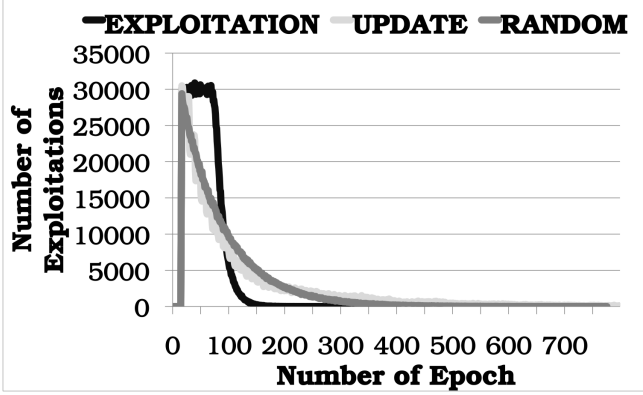

(d) Exploitation outcomes under asynchronous changes.

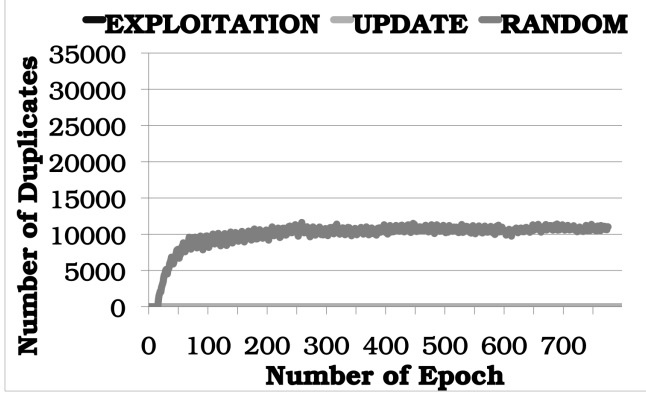

(f) Duplicate outcomes under asynchronous changes.

Figure 5.6: The number of outcomes for the EXPLOITATION, UPDATE and RANDOM adaptation strategies.

\subsubsection{Bloom filter aggregation memberships}

This section investigates the impact of false positives in the accuracy $a$ of the computed aggregates and the communication cost of DIAS. Specifically, the bloom filters scheme of $\mathrm{m}=16$ and $\mathrm{k}=24$ adopted in Section 5.8.2 is compared with two other schemes prone to false positives according to the empirical investigations: (i) $\mathrm{m}=14, \mathrm{k}=24$ and (ii) $\mathrm{m}=14$ and $\mathrm{k}=6$.

Concerning the accuracy of the computed aggregates, no significant influence is 


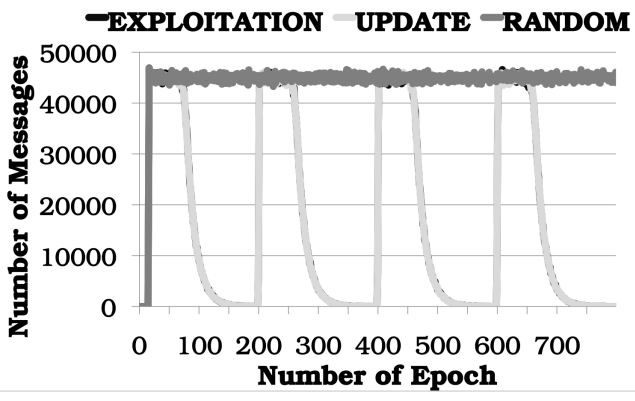

(a) Synchronous changes.

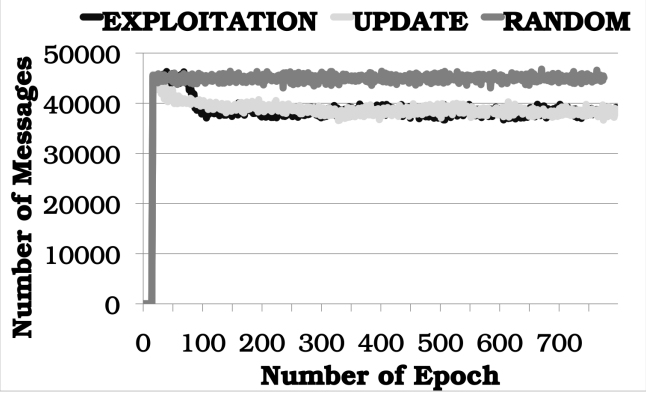

(b) Asynchronous changes.

Figure 5.7: Number of messages $\lambda($ sessions) exchanged during the aggregation sessions for the EXPLOITATION, UPDATE and RANDOM adaptation strategies.

observed in the two schemes prone to false positives. The matching $\mu$ between the computed and actual aggregates for both (i) aggregation strategies and (ii) synchronous/asynchronous changes remains almost intact. For example, the bloom filter scheme with $\mathrm{m}=14$ and $\mathrm{k}=24$ results in a 0.01 lower matching of AVERAGE under synchronous changes compared to the one with $\mathrm{m}=16$ and $\mathrm{k}=24$.

Figure 5.8 compares the outcomes of aggregation sessions for the EXPLOITATION adaptation strategy in each of the bloom filter schemes. The results of the UPDATE adaptation strategy are similar and, therefore, are omitted. The exploitation outcomes are also omitted as they show similar results to those of Figure $5.8 \mathrm{a}$ and $5.8 \mathrm{~d}^{8}$.

The bloom filter schemes with $\mathrm{m}=14, \mathrm{k}=24$ and $\mathrm{m}=14, \mathrm{k}=6$ miss update outcomes that actually result in inconsistency outcomes. This is shown in Figure 5.8a and 5.8b under synchronous changes and in Figure 5.8d and 5.8e under asynchronous changes. Figure $5.8 \mathrm{c}$ and $5.8 \mathrm{f}$ show that the scheme with $\mathrm{m}=14, \mathrm{k}=24$ generates 6 and 9 duplicate ${ }^{9}$ outcomes as a result of false positives during classification of aggregators in the aggregation pool.

False positives result in a higher number of messages $\lambda$ (sessions) generated by the adaptation strategies during runtime. This is because during the classification of aggregators false positives result in aggregation sessions of inconsistency outcomes. An aggregator $\mathrm{A}_{j}$ is incorrectly classified in the outdated and exploited views if the memberships $M_{D_{i}}\left(\mathrm{~A}_{j}\right)$ and $M_{S_{i}^{\prime}}\left(\mathrm{A}_{j}\right)$ are false positive respectively. Note also that when synchronous changes occur, incorrectly classified exploited aggregators move to the outdated view causing aggregation sessions that are inconsistent. Figure 5.9 illustrates the number of messages $\lambda$ (sessions) generated by the aggregation sessions of DIAS for the three bloom filter schemes during runtime. ExPLOITATION is the adaptation strategy adopted. The results of the UPDATE adaptation strategy are similar and, therefore, are omitted.

Inconsistency outcomes raise the total number of messages exchanged by $15 \%$. The same holds for asynchronous changes but the effect is much smaller as changes in the selected states occur more frequently. In this case, the increase in the total number of

\footnotetext{
${ }^{8}$ This is because exploitation outcomes are reached during convergence in which the bloom filters do not contain enough aggregation memberships to generate false positives. Recall that the probability of false positives increases as more memberships are stored in the bloom filters.

${ }^{9}$ Note that there may be more duplicate outcomes that cannot be detected and result in inconsistency outcomes.
} 


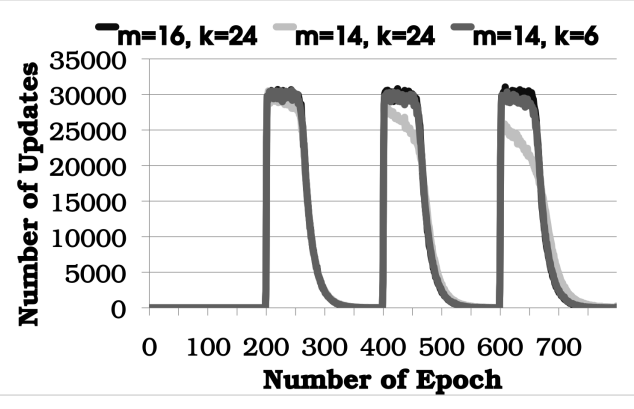

(a) Update outcomes under synchronous changes.

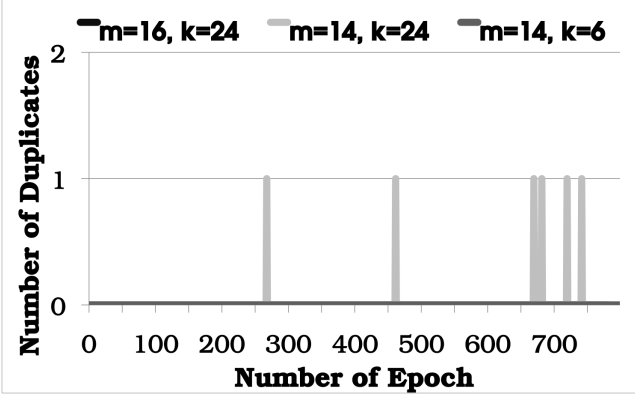

(c) Duplicate outcomes under synchronous changes.

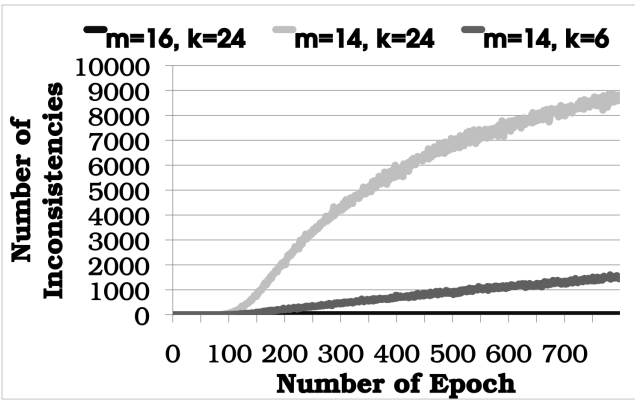

(e) Inconsistency outcomes under asynchronous changes.

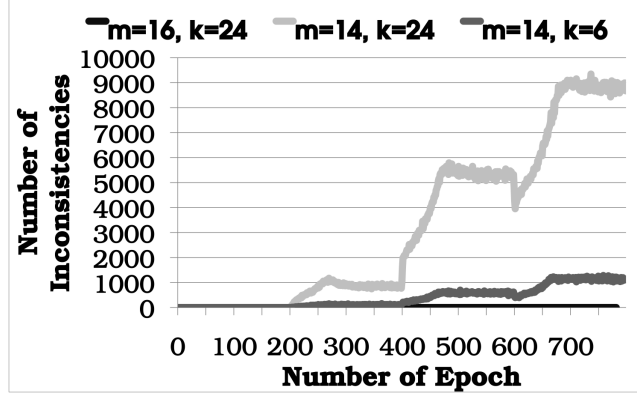

(b) Inconsistency outcomes under synchronous changes.

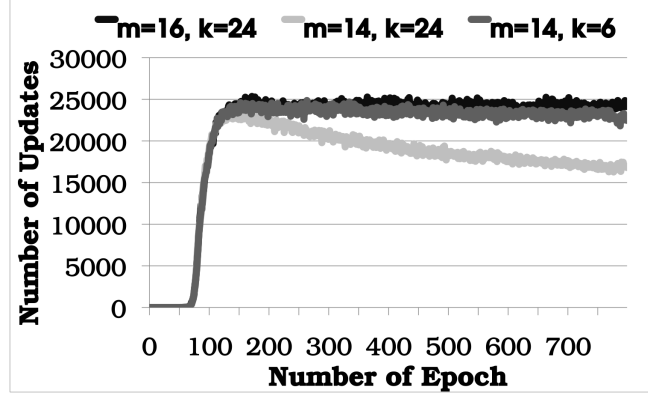

(d) Update outcomes under asynchronous changes.

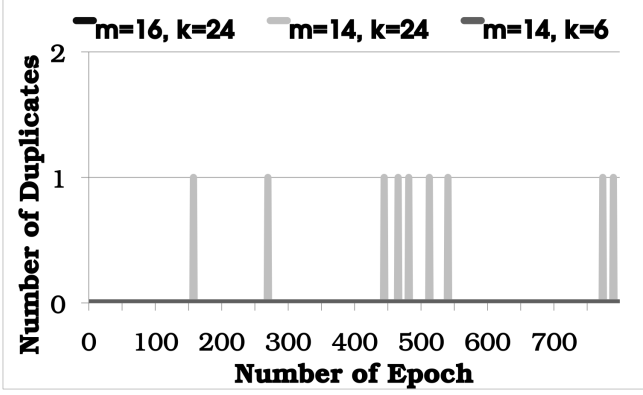

(f) Duplicate outcomes under asynchronous changes.

Figure 5.8: The number of outcomes for the EXPLOITATION adaptation strategy using three bloom filter schemes: (i) $\mathrm{m}=16, \mathrm{k}=24$, (ii) $\mathrm{m}=14, \mathrm{k}=24$ and (iii) $\mathrm{m}=14, \mathrm{k}=6$.

messages is $2 \%$.

\subsubsection{Adjusting the size of the aggregation pool}

Decreasing the size of the aggregation pool from $q=45$ to $q=30$, with a maximum number of 10 aggregators in each class does not have any significant influence in the accuracy $a$, matching $\mu$ and communication cost $\lambda$ (sessions) of DIAS. As long as the number of aggregation sessions is lower than the available aggregators in the aggrega- 


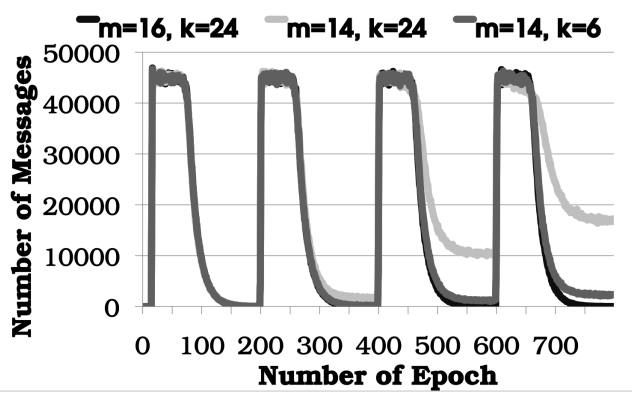

(a) Synchronous changes.

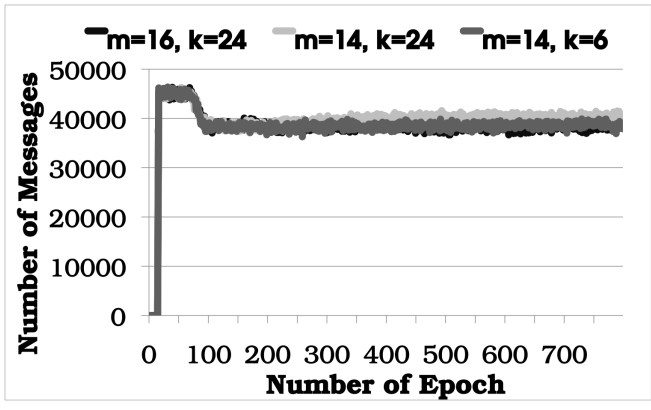

(b) Asynchronous changes.

Figure 5.9: Number of messages $\lambda($ sessions) exchanged during the aggregation sessions for the EXPLOITATION adaptation strategy using three bloom filter schemes: (i) $\mathrm{m}=16, \mathrm{k}=24$, (ii) $\mathrm{m}=14$, $\mathrm{k}=24$ and (iii) $\mathrm{m}=14, \mathrm{k}=6$.

tion pool, the size of the aggregation pool does not influence the performance of DIAS.

\subsubsection{Adjusting the size of the aggregator classes}

An unequal distribution in the size to the aggregator classes does not have a significant influence in the accuracy $a$, matching $\mu$ and communication cost $\lambda$ (sessions) of DIAS. This claim is supported by testing an aggregation pool with a maximum number of 25 unexploited, 5 exploited and 15 outdated aggregators.

\subsubsection{Adjusting the number of aggregator samples}

Decreasing the number of collected aggregator samples $e$ from 15 to 7 results in a decrease of 30 epochs in the convergence speed of accuracy $a$. The influence of matching $\mu$ is insignificant. The total communication cost for synchronous changes remains the same, however, its distribution is spread by 30 epochs caused by the convergence delay. In asynchronous changes and due to the continues changes in the aggregates, the communication cost is increased by approximately 2000 messages during convergence.

Performance is influenced as $e=7<z=10$ and therefore, there are epochs within which the aggregation pool does not contain enough aggregator samples to exploit all of the $z=10$ aggregation sessions.

\subsubsection{Adjusting the number of aggregation sessions}

The influence of $z$ in the performance of DIAS is similar to the influence of the number of aggregator samples collected from the discovery level. Yet, the number of aggregation sessions has an even higher impact in the performance of DIAS. The convergence speed is slower by 100 epochs if $z=5$ compared to $z=10$. The total communication cost for synchronous changes remains the same, however, its distribution spreads in a longer by 100 epochs period of time as Figure 5.10a depicts. In asynchronous changes, the communication cost is doubled as shown in Figure 5.10b. This observation suggests a performance trade-off between convergence speed and communication cost. 


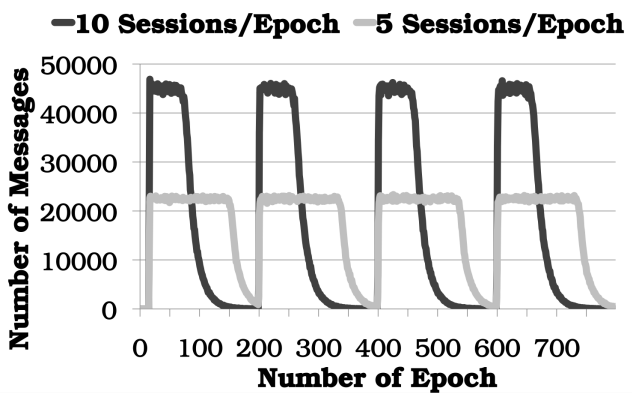

(a) Synchronous changes.

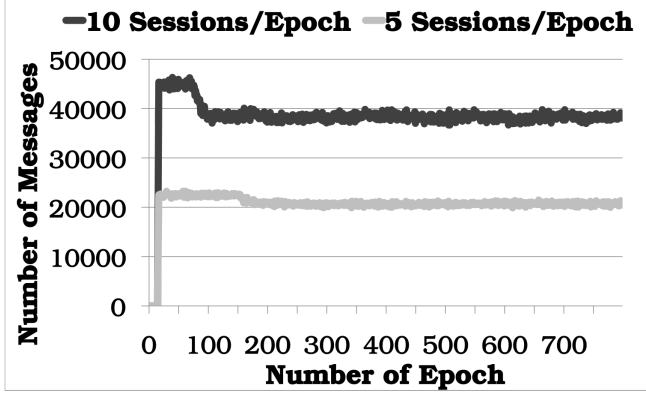

(b) Asynchronous changes.

Figure 5.10: Number of messages $\lambda$ (sessions) exchanged during the aggregation sessions of DIAS for the EXPLOITATION adaptation strategy using (i) $z=10$ and (ii) $z=5$.

\subsubsection{Adjusting the periodical executions}

The communication cost of the discovery level is constant and depends on the gossiping period of the peer sampling service [Jelasity et al., 2007]. The agents of the discovery level exchange periodically 2 messages as defined by this 'push-pull' gossiping protocol. Furthermore, gossiping is executed 4 times during an epoch as $T$ (discovery level) $=$ $T$ (DIAS) $/ 4=1000 / 4=250 \mathrm{~ms}$. Therefore, the total number of messages that an agent of the discovery level sends during an epoch is $2 * 4=8$ messages or $\lambda($ discovery level $)=$ $8 * 1500=12000$ messages for the total number of these agents in the network. The experiments performed confirm these estimations.

Assume that the rate of the communication cost in the discovery level requires a significant decrease, for example, $T$ (discovery level $)=1000 \mathrm{~ms}$. This results in four times fewer number of messages compared to $T$ (discovery level $)=250$. Assume also that the number of aggregator samples collected doubles from 15 to 30 as a configuration choice to minimize the impact of synchronizing the periodical execution of the discovery level with the upper levels. The experimental results in this case show a slower convergence by 50 epochs as a payed cost for decreasing the number of messages from 12000 to 3000 in the discovery level. Furthermore, the total number of messages $\lambda$ (sessions) under synchronous changes remains the same, yet, there is a small increase of 3000 messages under asynchronous changes during convergence.

\subsection{Evaluation Summary}

The experimental results of Section 5.8 show that the EXPLOITATION and UPDATE adaptation strategies of DIAS achieve a maximum accuracy of $a=1$ and a matching $\mu>0.5$ for the AVERAGE and MAXIMUM aggregation functions under synchronous/asynchronous changes. The convergence of accuracy can be achieved within 100 epochs. Under synchronous changes, RANDOM cannot follow the matching (up to 26\% lower) and accuracy convergence (150 additional epochs) of EXPLOITATION and UPDATE. Furthermore, under asynchronous changes, SUMMATION is challenging to compute, especially for UPDATE whose convergence of accuracy is influenced by the continuous changes of the selected states. This influence results in a convergence period of 450 epochs that is 
150 epochs more than RANDOM. Note that under synchronous changes, the ExPLOITATION and UPDATE adaptation strategies consume up to 45000 messages per epoch during convergence that are minimized to zero after convergence. This is not the case (i) for RANDOM, which is prone to duplicates and continuously consumes 45000 messages during runtime, and (ii) for the case of asynchronous changes that requires a continuous communication in order the agents to update their aggregates.

The above results are acquired with settings of bloom filters that do not generate false positives. If the settings of bloom filters generate false positives, the accuracy and matching remain almost intact. This is achieved by a significant number of inconsistency outcomes detected by DIAS that prevent inaccurate computations of aggregates. The number of inconsistency outcomes approaches the maximum of 9000 that are reached instead of update outcomes. These outcomes also cause $15 \%$ higher communication cost.

The size of the aggregation pool and the relative size of the aggregator classes do not influence the performance of DIAS. This is not the case for the number of aggregator samples collected from the discovery level. Performance degrades if the number of aggregator samples $e$ is lower than the number of aggregation sessions $z$ established every epoch. If $e=7<z=10$, the convergence speed of accuracy decreases by 30 epochs. In addition, the communication cost increases by 2000 messages during convergence for asynchronous changes. Moreover, if the number of aggregation sessions $Z$ decreases from 10 to 5, convergence speed decreases by 100 epochs. The total communication cost remains the same under synchronous changes, however it almost doubles under asynchronous changes.

Finally, any decrease in the frequency of periodical executions at the discovery level increases the communication cost of this level proportionally. Although the communication cost of the structuring level is not influenced under synchronous changes, an increase of 3000 messages is observed under asynchronous changes.

\subsection{Comparison with Related Work}

DIAS achieves a generic function-independent aggregation because of its fundamental design approach: the efficient representation and local storage of the distributed aggregation values as memberships of the final aggregates. DIAS performs explicit computations of aggregates in every peer of the network for each single distributed aggregation value collected. Such an abstraction results in a larger message overhead compared to related approaches based on the diffusion of the aggregation values. Table 5.2 summarizes the related aggregation mechanisms discussed in this section.

The iterative variance reduction algorithm of Jelasity et al. [2005] computes the AVERAGE approximately 4-5 faster than DIAS under static aggregation values. However, for synchronous changes, the performance of the two aggregation methodologies, i.e., communication cost in number of messages and convergence speed, becomes comparable as the iterative variance reduction algorithm requires recomputation of the aggregates. This performance impact becomes more significant as the frequency of changes increases, for example, more than 4-5 times faster convergence for DIAS. Furthermore, if changes become asynchronous, the algorithm of Jelasity et al. [2005] becomes infeasible. Recomputations of aggregates cannot be performed as they require some type of synchronization. Finally, DIAS does not require any changes in its aggregation method- 
Table 5.2: An overview of related decentralized mechanisms to DIAS.

\begin{tabular}{|c|c|c|c|c|}
\hline & $\begin{array}{l}\text { Aggregation } \\
\text { Function }\end{array}$ & $\begin{array}{l}\text { Aggregation } \\
\text { Values }\end{array}$ & $\begin{array}{c}\text { Routing } \\
\text { Requirements }\end{array}$ & $\begin{array}{c}\text { Storage } \\
\text { Requirements }\end{array}$ \\
\hline DIAS & any & highly dynamic & $\begin{array}{l}\text { dissemination } \\
\text { and collection }\end{array}$ & bloom filters \\
\hline $\begin{array}{l}\text { Nabeel Ahmed, } \\
\text { David Hadaller } \\
{[2006]}\end{array}$ & $\begin{array}{c}\text { SUMMATION }^{\mathrm{a}} \text {, } \\
\text { COUNT, AVERAGE, } \\
\text { STANDARD } \\
\text { DEVIATION }^{\mathrm{b}}\end{array}$ & dynamic & $\begin{array}{l}\text { flooding, } \\
\text { gossiping or } \\
\text { random walks }\end{array}$ & $\begin{array}{c}\text { counting } \\
\text { sketches [Flajolet } \\
\text { and Nigel Martin, } \\
\text { 1985] }\end{array}$ \\
\hline $\begin{array}{l}\text { Haridasan and } \\
\text { van Renesse } \\
{[2008]}\end{array}$ & $\begin{array}{l}\text { distribution of } \\
\text { aggregation } \\
\text { values }\end{array}$ & static & gossiping & synopsis diffusion \\
\hline $\begin{array}{l}\text { Jelasity et al. } \\
{[2005]}\end{array}$ & $\begin{array}{c}\text { AVERAGE, COUNT } \\
\text { SUMMATION }^{\mathrm{a}} \\
\end{array}$ & $\begin{array}{c}\text { static, } \\
\text { recomputations }\end{array}$ & gossiping & $\begin{array}{c}\text { hash maps for } \\
\text { COUNT }\end{array}$ \\
\hline $\begin{array}{l}\text { Kashyap et al. } \\
\text { [2006] }\end{array}$ & $\begin{array}{c}\text { algorithm } \\
\text { variations for } \\
\text { MINIMUM, MAXIMUM, } \\
\text { SUMMATION, } \\
\text { AVERAGE, RANK }\end{array}$ & static & $\begin{array}{l}\text { group formation } \\
\text { and gossiping }\end{array}$ & synopsis diffusion \\
\hline $\begin{array}{l}\text { Kempe et al. } \\
\text { [2003] }\end{array}$ & $\begin{array}{l}\text { algorithm } \\
\text { variations for } \\
\text { SUMMATION, } \\
\text { AVERAGE and } \\
\text { quantiles }\end{array}$ & static & gossiping & synopsis diffusion \\
\hline Nath et al. [2008] & SUMMATION, COUNT & static & $\begin{array}{l}\text { ring/tree } \\
\text { topologies, } \\
\text { flooding }\end{array}$ & synopsis diffusion \\
\hline $\begin{array}{l}\text { Ogston and } \\
\text { Jarvis [2010] }\end{array}$ & $\begin{array}{c}\text { SUMMATION }^{\mathrm{d}} \\
\text { queries }^{-}\end{array}$ & dynamic & tree topology & $\begin{array}{c}\text { parent and } \\
\text { children }\end{array}$ \\
\hline
\end{tabular}

ology if different aggregation functions need to be computed simultaneously. Note that this is the most cost-effective use of DIAS that motivates its selection for aggregation over related methodologies.

Diffusion methodologies cannot be applied to a wide range of aggregation functions and are usually routing-dependent. For example, MAXIMUM and Minimum require the communication cost of epidemics [Jelasity et al., 2005, Kashyap et al., 2006] that approaches the one of DIAS. The COUNT aggregate requires additional computational and storage complexity to effectively apply the 'inverse birthday paradox' [Jelasity et al., 2005] based on which the SUMMATION aggregate is computed.

Furthermore, the information diffusion and gossiping aggregation of Haridasan and van Renesse [2008], Jelasity et al. [2005], Kempe et al. [2003], Kennedy et al. [2009] and Nath et al. [2008] do not consider dynamic changes of the aggregation values and assume synchronized recomputations. Coordination of these recomputations in distributed environments in not straightforward. Nabeel Ahmed, David Hadaller [2006] extend the work of Nath et al. [2008] on synopsis diffusion by incorporating incremental updates of aggregates if changes in the aggregation values occur. However, only a relatively low number of changes can be tolerated compared to DIAS. For example, DIAS 
tolerates in the illustrated experiments 33600 changes compared to the 1000 changes in the work of Nabeel Ahmed, David Hadaller [2006]. This is because the bit vectors of synopsis diffusion cannot tolerate the representation of a high number of items as it causes significant inaccuracies. The false positives of DIAS do not influence the accuracy of aggregates as they can be detected and eliminated by the mutual membership checks.

Robust tree overlays are a flexible methodology to compute a wide range of aggregates but require topology self-management [Pournaras et al., 2010a,b] in decentralized environments as shown in Chapter 4. Communication and storage complexity can be higher than the aggregation itself, if such mechanisms are used explicitly for aggregation. Performing a relevant evaluation and comparison of aggregation trees with other more dedicated to aggregation mechanisms, such as DIAS, requires a use-case context and a specific application scenario. In contrast, if tree topologies are reused between different distributed applications, including aggregation, the allocated cost of the overlay service is shared between these applications something that makes the use of trees more effective as illustrated by Fei et al. [2001]. The unique paths of tree topologies are not required in DIAS as unique aggregation values are identified by the classification performed in the structuring level. Furthermore, tree aggregation suffers from an unequal load distribution in nodes and the impact of failures as discussed by Ogston and Jarvis [2010]. The nodes close to the root receive a high number of forwarded messages from the bottom nodes. Similarly, the impact of a failure close to the leaves is small whereas a single failure close to the root partitions the whole overlay network. These issues do not concern DIAS as it does not depend on a specific routing mechanism. Nonetheless, the realization of the discovery level by the peer sampling service [Jelasity et al., 2007] results in a uniform communication overhead between nodes.

In general, DIAS achieves a dynamic function-independent and routing-independent aggregation because of its fundamental design approach: the efficient representation and local storage of the distributed aggregation values as memberships of the final aggregates. DIAS performs explicit aggregate computations in every aggregator of the network for each single distributed aggregation value. Such an abstraction results in a relative large message overhead compared to related approaches based on the 'mass diffusion' of aggregation values.

\subsection{Discussion and Future Work}

DIAS is a realization of the ASMA architecture that provides three levels of abstraction and modularity in the aggregation process. The coordination level does not have any knowledge about the underlying complexity of the classification and aggregation memberships. A wide range of aggregation functions can be accurately computed as the structuring level guarantees that the provided aggregator samples are classified as unexploited or outdated. Similarly, the structuring level receives random aggregator samples from the discovery level. Beyond the peer sampling service, other mechanisms can realize the discovery level such as flooding [Jiang et al., 2003] and random walks [Gkantsidis et al., 2006].

A key feature of DIAS is the predefined number of possibles states during an aggregation phase. There are at least two reasons that this feature does not limit the approach of DIAS. The first reason concerns the fact that a large number of applications are fundamentally based on this assumption and design. User ranking aggregation in 
recommender systems, such as the ones of Garcin et al. [2009] and Lekakos and Giaglis [2007], is based on a finite and often restricted number of options for a user to rank an element. Applications of demand-side energy management, such as the one of James et al. [2006] and the work [Pournaras et al., 2010c, 2009b] illustrated in Chapter 6, aggregate information about a finite number of alternative energy consumptions of home devices to improve the stability of the Smart Power Grid. The second reason is related to the potential to extend the idea of discrete possible states to continuous ranges of values from which possible states are parameterized. In this case, each possible state is a representative value, e.g., average, of a respective range of values.

Dissemination and collection of all aggregation values in every agent of the network requires a significant communication cost in DIAS. Section 5.10 shows that this cost is relatively higher than related aggregation methodologies. One way to decrease this cost is to eliminate the number of aggregators and disseminators in a network. Section 5.8.2 shows that the communication cost of DIAS is decreased more than half if the network is split into the $2 / 3$ of the peers running disseminators and $1 / 3$ aggregators. It is not always necessary for each peer of the network to perform both aggregation and dissemination as various applications do not require this. Section 6.4 discusses an application scenario in the energy domain in which agents have different goals and require aggregators or disseminators but not both. Limiting the number of aggregators or disseminators is a way to decrease the communication cost significantly.

DIAS is based on the exchange of aggregator samples instead of disseminator samples. In the current design of DIAS, aggregation values are disseminated to aggregators instead of the aggregators requesting the aggregation values. The $M_{\mathrm{A}_{i}}\left(\mathrm{~S}_{j}^{\prime}\right)$ membership of the aggregator cannot be used during the classification process as the selected state $\mathrm{S}_{j}^{\prime}$ is not known. This issue can be overcome by injecting the selected state in the exchanged disseminator samples of the discovery level.

Experimental evaluation illustrates the efficiency of DIAS under different experimental settings. High accuracy and matching is achieved even in the case of false positives in the bloom filters. The tolerance of DIAS to false positives provides some large data space savings. Accuracy is maintained even in the case of significantly decreasing the size of bloom filters, resulting in a high number of false positives that DIAS detects. The size of the bloom filters is examined in this chapter empirically. However, a future extension is the dynamic and automated allocation of larger space in the bloom filters based on accuracy requirements under false positives. Furthermore, alternative approaches to bloom filters are also considered in future work, e.g., hash compaction [Dillinger and Manolios, 2004].

The classification of aggregator samples in the aggregation pool proactively prevents duplicate outcomes that increase the communication overhead. Moreover, the mutual membership checks reactively detect duplicate outcomes that are not detected in the classification due to false positives. Therefore, mutual membership checks guarantee a high accuracy in the computation of aggregates, especially in the case of duplicatesensitive aggregation functions such as the SUMMATION without introducing additional communication cost. The performance of the RANDOM strategy shows the large communication cost that duplicate outcomes cause and the large savings achieved by the EXPLOITATION and UPDATE adaptation strategies. Furthermore, EXPLOITATION and uPdATE cover a wide range of network settings. For example, during network scaling by peer arrivals or during bootstrapping, EXPLOITATION provides a faster convergence of accuracy. In a stable network with continuous changes of selected states, UPDATE provides a 
faster adaptation of the aggregates. An automated switch of strategies is possible. The illustrated results in Section 5.8.2 depict the convergence of the exploitation and update outcomes based on which a decision for switching an adaptation strategy can be made by agents.

Other future work concerns the evaluation of DIAS and its applications in various network conditions, such as churn [Kennedy et al., 2009] and latency.

\subsection{Conclusions}

This paper concludes that DIAS, as an overlay service of the ASMA architecture, is able to act as a generic and decentralized middleware for dynamic aggregation in largescale distributed networks. The aggregation approach of DIAS is holistic: a local and duplicate-free availability of the distributed aggregation values that enables the simultaneous computation of almost any aggregation function. Achieving this abstraction in a cost-effective manner and without depending on a specific routing mechanism is a challenge that has not been addressed in related work. DIAS meets these requirements by introducing an implicit representation and storage of the explicit distributed aggregation values: aggregation memberships in bloom filters. However, the high degree of abstraction in DIAS has an impact of a higher communication overhead compared to methodologies based on information diffusion [Jelasity et al., 2005, Nath et al., 2008].

The experimental evaluation shows that DIAS achieves a high accuracy under synchronous and asynchronous changes of the aggregation values. Even under challenging settings of bloom filters with a high number of false positives, accuracy is maintained almost entirely due to the mutual membership checks. The classification of aggregator samples and their selection based on two adaptation strategies provide (i) the minimization of duplicates that increase inaccuracies and communication overhead and (ii) the intelligent adaptation of the aggregation process in different network conditions. 



\section{Chapter 6

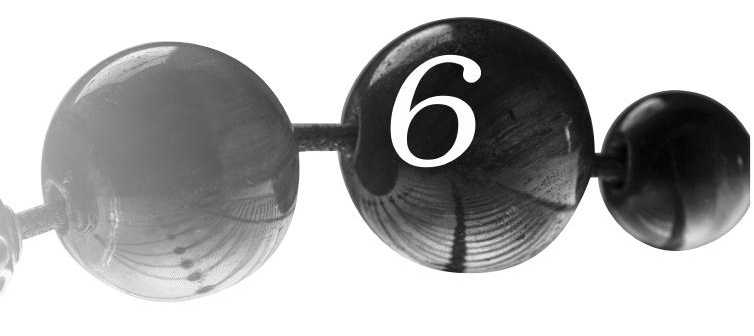

\section{Demand-side Energy Management using Overlay Services*}

"Make the best use of what is in your power, and take the rest as it happens."

Epictetus $^{1}$

Meeting robustness criteria in socio-technical infrastructures, such as the Smart Power Grid, is increasingly complex and challenging [Ipakchi and Albuyeh, 2009]. For example, the variation of power demand [Strbac, 2008], the outdated infrastructure [Massoud Amin and Wollenberg, 2005], the integration of renewable energy resources [Brandstätt et al., 2011] and technologies such as electrical vehicles [ClementNyns et al., 2010] are some of these emergent challenges. Traditionally, matching supply and demand has been managed from production-side that usually involves complex and costly actions managed by human actors, e.g., system operators. Such actions include the activation of operating reserves and the installation of new power plants or other infrastructure [Joskow and Tirole, 2007].

Within an electrical power grid that is smart, sustainable, environmental-friendly and has the means to self-configure its load in extreme and unpredictable conditions, demand-side energy management is an alternative promising approach. Two demandside energy management mechanisms based on overlay services are introduced in this chapter: (i) EPOS, the Energy Plan Overlay Self-stabilization system and ALMA, the Adaptive Load Management by Aggregation system. These mechanisms provide an intelligent communication and interaction medium to consumers that can be used to collectively influence the aggregate energy consumption under different conditions.

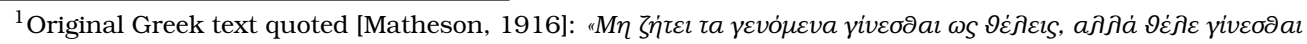

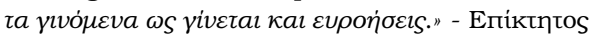

${ }^{*}$ This chapter is based on five published papers [Pournaras et al., 2010d,c, 2009b, 2008c,b] and a submitted paper [Pournaras et al., 2013b]. 
EPOS performs stabilization of energy consumption over time by minimizing deviation and reversing deviation of earlier energy consumption. This is achieved by software agents that control thermostatically controlled appliances, i.e., refrigerators, water heaters etc. Agents self-organize themselves in a tree overlay network to coordinate energy consumption of their devices. Self-organization is performed using the AETOS overlay service illustrated in Chapter 4. Coordination is automated and based exclusively on the operational flexibility of thermostatically controlled appliances. The involvement of consumers is not required.

However, in extreme conditions in which the Smart Power Grid requires an actual increase or decrease of demand due to a failure of a power plant or excessive availability of micro-generation [Paulus and Borggrefe, 2011], ALMA can be used. Consumers dynamically select between a number of predefined demand options that represent comfort and economy levels of their energy consumption. Their selections are based on price or other incentives provided by utility companies. The DIAS overlay service makes the aggregate power demand locally available to consumers to trigger the required adjustments of energy consumption.

EPOS and ALMA are two highly decentralized mechanisms for demand-side energy management that provide two incremental levels of involvement and participation to consumers. This flexibility extends the applicability and scope of EPOS and ALMA compared to related approaches [Kok et al., 2005, Stadler et al., 2009, Hammerstrom, 2007, Faruqui and George, 2005, Hopper et al., 2006, Lu et al., 2005, Middelberg et al., 2009]. Experimental evaluation of EPOS and ALMA using both synthetic data and data collected from the Olympic Peninsula Smart Grid Demonstration Project [Hammerstrom, 2007] shows that decentralized stabilization and adjustment of energy consumption is possible.

This chapter is outlined as follows: Section 6.1 introduces the concept of demandside energy management in the Smart Power Grid and illustrates the research focus of this chapter. Section 6.2 summarizes the applicability of the AETOS and DIAS overlay services in two demand-side energy management mechanisms. Section 6.3 illustrates the first of these mechanisms, EPOS, the Energy Plan Overlay Self-stabilization. Section 6.4 studies the second mechanism for demand-side energy management: ALMA, Adaptive Load Management by Aggregation. Section 6.5 compares EPOS and ALMA with related work on demand-side energy management. Section 6.6 discusses EPOS and ALMA and outlines future work. Finally, Section 6.7 concludes this chapter.

\subsection{Demand-side Energy Management}

This section provides necessary research background on demand-side energy management and outlines the research focus of this chapter.

\subsubsection{Research background}

Demand-side energy management usually concerns (i) load-shifting of energy consumption at different time points and/or (ii) load-adjustment by increasing or decreasing overall energy consumption [Stadler et al., 2009, Strengers, 2008, Ashok, 2006]. Although demand-side energy management is not a recently introduced idea [Schweppe et al., 1989], nowadays it is an established operation of the Smart Power Grid because of 
the broad adoption of micro-generation using renewable energy resources and enabling control technologies in households such as smart sensors.

In the Smart Power Grid, residential consumers have a more interchangable ${ }^{2}$ role between consumer and producer as they have the option to perform a wide range of actions such as (i) sell their generated energy to the power grid, (ii) consume it locally or (iii) store it, for example, in batteries. Groups of such consumers may form virtual power plants that can provide operational flexibility to match supply and demand in several unexpected conditions [Dimeas and Hatziargyriou, 2007], i.e., failures of power lines or high peak loads. Wide deployment of solar panels and wind generators at the residential-level creates situations in which micro-generation exceeds demand resulting in higher risks of system blackouts as discussed by Brandstätt et al. [2011] and Stodola and Modi [2009] for the case of the German power grid. Similarly, wide deployment of new emerging technologies, such as electric vehicles, have a significant impact on energy consumption patterns and their prediction [Hadley and Tsvetkova, 2009, Clement-Nyns et al., 2010]. All of these issues make coordination of energy consumption in the Smart Power Grid crucial.

Demand-side energy management is orchestrated in practice by power utility companies via demand-response programs. An outline and review of existing demandresponse programs is illustrated by Albadi and El-Saadany [2008] and Cappers et al. [2010]. These programs include the installation of smart sensors, controllers and thermostats in the households of consumers to extract almost real-time information about the energy consumption used in order to control it. Home automation technologies such as the ones reviewed by Kailas et al. [2012] provide the following load management options:

- Adjustment of temperature setpoints in thermostatically controlled devices [Lu et al., 2005], e.g., water heaters, refrigerators or HVAC systems.

- Turning on/off consumption sources [Clement-Nyns et al., 2010], e.g., the charging of electrical vehicles.

- Adjustment of potentiometers that control consumption sources such as lighting [Alahmad et al., 2011].

- Hibernating monitors and personal computers [Ponciano and Brasileiro, 2010].

Note that demand-side energy management can also be applied in various domains of industrial consumers as illustrated by Paulus and Borggrefe [2011], Ashok [2006] and Middelberg et al. [2009].

Consumers require incentives to accept the idea that their energy consumption is controlled by automation technologies under certain circumstances [Strbac, 2008, Faruqui and George, 2005, Hopper et al., 2006]. Environmental concerns are not always adequate to guarantee a high level of participation and engagement [Hammerstrom, 2007, Sundramoorthy et al., 2011]. Incentives should be provided mainly by utilities via demand/response programs [Albadi and El-Saadany, 2008, Cappers et al., 2010]. However, energy policies may enforce a certain or minimum level of engagement such as the policies of the UK government that mandate all British households equipped with smart meters by 2020 [Sundramoorthy et al., 2011]. An incentive may concern a

\footnotetext{
${ }^{2}$ In literature, these consumers are referred to as prosumers [Grijalva and Tariq, 2011]
} 
benefit of lower pricing or other economic revenue offered as a result of allowing a level of control in the energy consumption for a predefined period of time that is negotiated between the consumers and their utility companies. A number of existing contract and pricing schemes are illustrated by Palensky and Dietrich [2011].

Consumer participation should be on a voluntary basis to a certain degree, meaning that the comfort of consumers is a non-negotiable right [Strengers, 2008]. In practice, consumers may have the option to overwrite the adjustments performed by the automated technologies. The concept of adjustable autonomy [Scerri et al., 2002] is highly applicable in this domain and such an option has been an adopted practice by both consumers and utility companies in the Olympic Peninsula Smart Grid Demonstration Project [Hammerstrom, 2007] and the statewide pricing pilot experiment of California [Faruqui and George, 2005]. Similarly, the Dehems ${ }^{3}$ system is a pilot project that tests and evaluates the user participation and engagement in realistic innovation platforms referred to as 'living labs'. These platforms bring together and involve end-users, researchers, industrialists and policy makers to examine a wide range of influencing factors such as (i) policies, (ii) behavioral context, and (iii) design concerns for the persuasive feedback and home technologies [Richardson, 2008, Sundramoorthy et al., 2011].

Demand side energy management usually introduces two levels of control: (i) local control by the consumer and (ii) global control by utility companies. Local control concerns changes of consumption behavior via awareness about energy consumption and the price the consumer pays. Furthermore, local control provides interfaces to certain households devices, such as heating, ventilation and cooling (HVAC) systems, for the configuration of their operation. In contrast, global (centralized) control may be directly applied by the utility companies via, for example, frequency signals to which household devices respond [Stadler et al., 2009] or indirectly via price incentives in order consumers to change their consumption behavior [Hammerstrom et al., 2010].

Local control cannot always address system objectives, such as minimization of unexpected power peaks, as consumers are unaware of the global state of the system. In contrast, global control requires continuous aggregation of each local information in a centralized management entity belonging to a utility company. This approach raises scalability, fault-tolerance and privacy issues. Utilities process information from millions of consumers and their devices in almost real-time. This increases the costs of utility companies that require investments in expensive storage, computation and communication facilities, e.g., data centers. Furthermore, centralization results in single points of failure that can be usually avoided by investments in additional computer facilities for redundancy. Finally, data centralization and management by utility companies raises privacy issues. Such detailed energy consumption data can be used to extract information about the lifestyle and activities of consumers as discussed by Lisovich et al. [2010] and AlAbdulkarim and Lukszo [2010]. Therefore, an important question is if detailed consumers' data may be stored and managed at the supply-side as utility companies mainly require aggregated energy consumption for the purpose of demand-side energy management and not a detailed real-time information about local consumption of individual consumers.

\footnotetext{
${ }^{3}$ Accessible at: http: / /www. dehems.eu/ (last accessed: May 2012)
} 


\subsubsection{Research focus}

This chapter focuses on the problem of how demand-side energy management can be applied in a decentralized fashion with respect to different required automation levels.

A demand-side energy management system is decentralized if it enables consumers to play an active role in the Smart Power Grid by autonomously controlling their energy consumption and production with minimum interventions from supply-side. Utility companies do not have detailed energy consumption data but only aggregated data. However, utility companies are able to feed consumers with the aforementioned system objectives via high-level policies, incentives, and pricing schemes.

The automation level of a demand-side energy management system defines a minimum involvement, participation and interaction of human consumer actors with demand-response programs of an energy management system. Software agent technologies [Scerri et al., 2002, Kok et al., 2005, Kailas et al., 2012] installed in sources of household consumption and production are the technical means to make autonomous control possible.

Decentralization and automation transform the problem of demand-side energy management to a large-scale agent-based coordination problem. Agents representing consumers and controlling their consumption devices may interact with each other in a peer-to-peer fashion and without centralized mediation to collectively coordinate their energy consumption and meet global objectives of the electrical power grid. Example of these objectives include minimization of power peaks, referred to as 'peak shaving', matching consumption patterns to the availability of renewable resources, or shifting energy consumption at different time.

\subsection{Overlay Services for Energy Management}

This chapter studies decentralized and automated demand-side energy management using overlay services. The applicability of AETOS and DIAS overlay services is illustrated through two demand-side energy management mechanisms introduced for this purpose: EPOS, the Energy Plan Overlay Self-stabilization and ALMA, the Adaptive Load Management by Aggregation.

EPOS performs stabilization of energy consumption without requiring human intervention and ideally without influencing the comfort and lifestyle of consumers. EPOS is based on large-scale coordination of software agents installed in thermostatically controlled appliances of consumers, e.g., refrigerators, water heaters, etc. Agents locally generate a set of possible (energy) plans that represent alternative energy consumption patterns for a future period of time and a specific device. The possible plans are assumed equivalent from the consumer's viewpoint. This means that the comfort impact on consumers between the possible plans is negligible. In practice, these alternative and equivalent possible plans are achieved by preheating/postheating or precooling/postcooling. Agents coordinate their selections about which plan to execute in order to achieve a collective stabilization of the energy consumption. EPOS is able to achieve two stabilization objectives that adjust the deviation (oscillations) of energy consumption: (i) Minimization of energy deviation and (ii) reverse of energy deviation. The first objective contributes to the maintenance of robustness in the Smart Power Grid as discussed by Strbac [2008] and Shaw et al. [2009]. The second objective captures 
the robustness of the Smart Power Grid in case of sudden power peaks or large-scale micro-generation by consumers. Coordination in EPOS is based on the communication topology of tree overlay networks that are self-organized by the AETOS overlay service. This chapter studies the effect of the tree topology in the performance of EPOS.

The stabilization achieved with EPOS has its limits as it is bound to the technical characteristics of the thermostatically controlled appliances managed. Load-shifting at different time points may not be adequate at situations in which the Smart Power Grid may experience, for example, failures of power generators. Consumers need to decrease their energy consumption in such cases till the system is back to its normal operation. The ALMA mechanism fills exactly this gap.

ALMA relies on behavioral flexibility that consumers can offer based on incentives provided by their utility companies. Consumers, via software agents, control home devices and configure a level of comfort and economy in their local energy consumption. Under extreme cases in which the Smart Power Grid is stretched by high load, consumers sacrifice a degree of comfort, on a voluntary basis, to obtain an economic or other revenue from their utility companies. Agents require awareness of the total energy consumption in a system to adapt their local energy consumption accordingly. This awareness is achieved via decentralized aggregation of information about energy consumption without the involvement of utility companies. ALMA is based on the DIAS overlay service as it enables multiple utilities and consumers to coexist in an aggregation overlay network and exchange information about the dynamically adaptive energy consumption. The feasibility of ALMA is validated by analytical results computed using data from an operational Smart Power Grid.

\subsection{Energy Plan Overlay Self-stabilization}

EPOS performs demand-side energy management by stabilizing global energy utilization. In this section, energy utilization is defined as the allocated amount of energy consumed by thermostatically control devices or stored to batteries for a period of time. Global energy utilization entails aggregation of all energy utilizations for the same time period. Finally, stabilization is the state in which the acquired global energy utilization maintains the Smart Power Grid robust, i.e., matching supply and demand, reducing production costs, etc.

EPOS manages the energy utilization of thermostatically controlled appliances, such as refrigerators, air conditioners and water heaters. These devices consume $25 \%$ of the total residential energy supply in the USA according to Kelso and Bruce [2001] and Moon and Han [2011], therefore, their management can have a significant impact on the stabilization of the Smart Power Grid. Furthermore, the management of thermostatically controlled appliances can be automated without requiring direct control by consumers. In contrast to other consumption sources such as lighting or personal computers, management of thermostatically controlled appliances does not have a significant impact on consumers. For example, turning off lights automatically may result in an effect of disturbance for consumers. In contrast, turning off the compressor of a refrigerator for a short period of time does not necessarily disturb its intended operation.

The stabilization objectives that EPOS is able to achieve are the following:

- Minimum deviation: The system proactively minimizes deviation in the global energy utilization. Power peaks are suppressed and energy consumption oscillates 
to a minimum degree.

- Reversed deviation: The system reactively reverses consumption deviation of the global energy utilization with respect to a secondary global energy utilization. The average result of the two global utilizations should be 'flat' over time.

Stabilization according to the above objectives has an impact on the robustness of the Smart Power Grid as illustrated in the following examples: (i) Minimization of deviation aims to maintain the energy consumption to a steady level without significant oscillations and power peaks. This is a fundamental operation required in every power grid. (ii) The reverse of deviation is applicable in a number of scenarios. One of them is when a sudden peak in the energy consumption results in an increase of the energy provided by a power plant. Reversing high energy consumption to a lower energy consumption in the next utilization period keeps the cost of the energy provided by this power plant balanced. A second application is micro-generation by consumers. Batteries can be used as an energy buffer for protection of the Smart Power Grid. The battery utilization level should remain stable over time. For example, a battery charged steadily at $40 \%$ allows consumers to draw critical power during a power outage. Furthermore, the remainder $60 \%$ capacity available in the battery can be used as protection storage for the Smart Power Grid when micro-generation exceeds demand. Therefore, the battery level should remain constant and any deviation should be reversed by controlling energy consumption.

EPOS achieves these objectives by managing thermostatically controlled appliances of consumers using software agents [Vidal et al., 2001] that communicate through an infrastructure such as the Internet or an integrated communication system in the Smart Power Grid. An example of the latter communication scheme is illustrated by Stadler et al. [2009]. These communication options are realistic according to the work of Guo et al. [2005], James et al. [2006], Hammerstrom et al. [2010] and Kailas et al. [2012].

EPOS exploits local alternative energy utilizations based on which thermostatic devices can operate. These devices work in a periodic fashion by turning the thermostat 'on' and 'off'. In this way, the temperature of the devices remains within the target range of its operation. Temperature for which the thermostat changes from the 'on' state to the 'off' state, and visa versa, is referred to as temperature setpoint. Note that thermostatic devices consume energy during the 'on' state.

The intuition behind EPOS is that software agents can modify the temperature setpoints and change the energy utilization over time by keeping the target temperature range unmodified. In this way, the consumer does not experience any significant inconvenience or discomfort. The software agents of EPOS generate one or more energy utilization plans for a given period of time. Each plan consists of a sequence of discreet energy values. Each value represents a level of energy utilization at a specific time point. For example, the plan $\mathbf{x}=\{5.3,6.4,6.1,8.6,8.1,4.2\}$ represents the utilization of energy for $|\mathbf{x}|=6$ consecutive equal time periods. During the third time period, the plan utilizes 6.1 units of energy. A set of $\mathrm{v}$ possible (energy) plans represents different energy utilization options for a single device during a given period of time. This notion of energy plan is used throughout this section. The generation of alternative energy utilization and the flexibility of thermostatic devices is a technically viable approach for demand-side energy management as it is shown by Lu et al. [2005]. Figure 6.1 depicts the idea of generating different possible plans for a thermostatic device such as a water heater. 


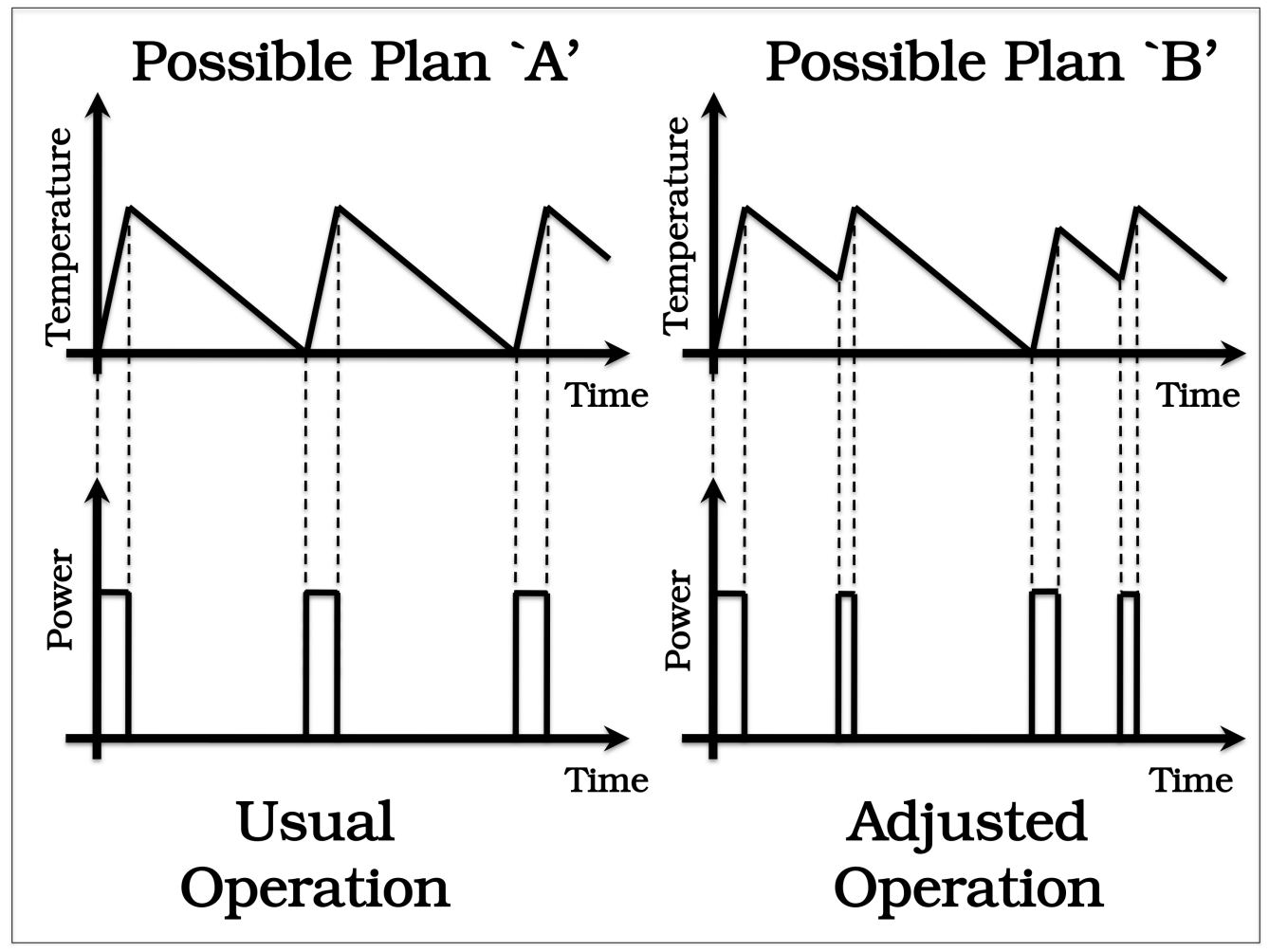

Figure 6.1: A simplified illustration of two possible energy plans. Possible plan ' $A$ ' is a conventional one that always uses the same temperature setpoints. In contrast, possible plan 'B' is generated by modifying the temperature setpoints. The power distribution of plan ' $\mathrm{B}$ ' over time is different than the one of plan 'A'.

Each agent in EPOS chooses the selected (energy) plan to execute between the set of possible plans. This selection is an actual thermostat configuration for the time period defined by the plan. Note that a global plan corresponds to the aggregation (summation) of all of the selected plans of the participating agents in EPOS. The optimum solution in this problem can be found using centralized coordination. This approach includes the brute-force computation that sums up all combinations of possible plans of each agent in the system. These computed combinations are referred to in this chapter as combinational plans. Centralized coordination guarantees the discovery of the best global solution, considering and assuming that the sets of possible plans do not change during coordination. However, centralized coordination based on brute-force operation does not scale. The complexity is $O\left(\mathrm{v}^{n}\right)$, where $\mathrm{v}$ is the number of possible plans per agent and $n$ is the number of agents in the network. Alternatively, EPOS performs decentralized coordination that results in a global plan that is more stabilized compared to a system in which agents always determine their energy consumption locally (without coordination). This chapter refers to this default and locally selected energy consumption as intended.

Coordination of energy consumption in EPOS is performed by a decentralized aggregation and decision-making mechanism that relies on self-organization of agents in a 
hierarchical topology: a tree overlay network. The remainder of this section illustrates the coordination mechanism of EPOS and the use of the AETOS overlay service. Furthermore, experimental evaluation of EPOS shows the performance achieved for each stabilization objective under various experimental settings.

\subsubsection{Coordination of energy consumption}

Assume a network of communicating agents that control thermostatically controlled appliances of consumers. These agents are organized in a tree overlay network using the overlay service of AETOS. More information about this organizational and design choice is provided in Section 6.3.2. This section focuses exclusively on the coordination mechanism. Assume that each agent in the tree overlay has a number of $k$ children, except the leaves that do not have any children. Each agent generates a number of $v$ possible plans.

Algorithm 6.1 illustrates the execution of EPOS for each agent based on the tasks below. These agent tasks perform the coordination of energy consumption in EPOS. More specifically, coordination is performed incrementally for each two consecutive levels of the tree overlay network. A level of children interacts with their parents in the level above. A coordination step is defined by the tasks that result in the selection and execution of selected plans in the level of children. In addition, a coordination phase is defined by recursive coordination steps for the total number of tree levels in the overlay network. The transition from one coordination phase to the next one is realized by the broadcast task.

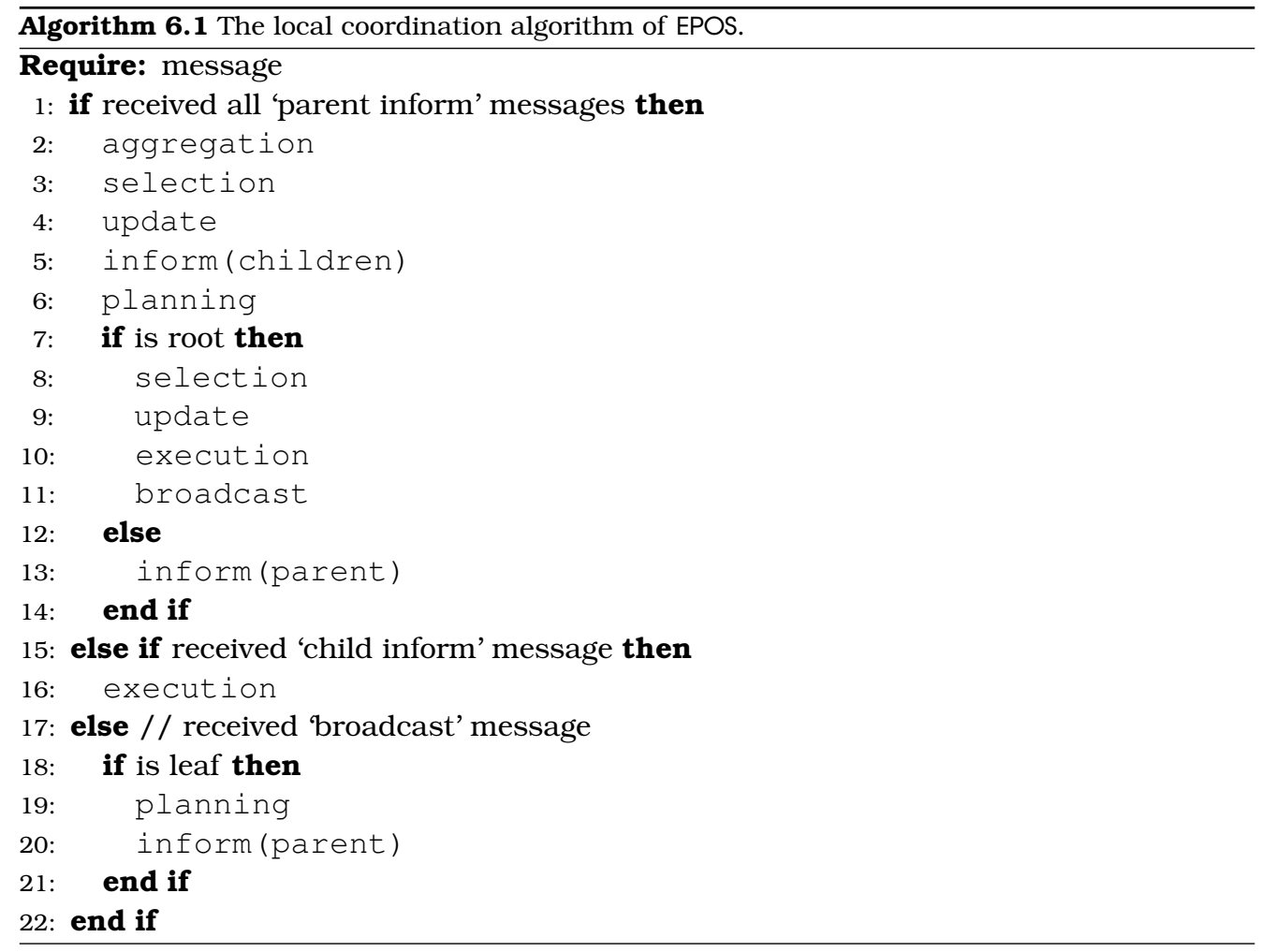


- planning: Each agent $i$ locally generates a set $\mathbf{P}_{i}=\left\{\mathbf{p}_{i}^{0}, \ldots, \mathbf{p}_{i}^{\mathrm{v}-1}\right\}$ of $\mathrm{v}$ possible plans for a future period of time.

- inform (parent): Following planning, each agent $i$ sends to its parent the set $\mathbf{P}_{i}$ of possible plans and the aggregate plans $\mathbf{a}_{i}$ and $\hat{\mathbf{a}}_{i}$. An aggregate plan is the summation of all of the selected plans by the agents that belong to the tree branch underneath agent $i$. The aggregate plan $\mathbf{a}_{i}$ concerns the current coordination phase, whereas, the aggregate plan $\hat{\mathbf{a}}_{i}$ refers to an earlier coordination phase.

- aggregation: Each agent $i$ collects the possible and aggregate plans from each of its children. It computes all of the combinational plans $\mathbf{C}_{i}=\left\{\mathbf{c}_{i}^{0}, \ldots, \mathbf{c}_{i}^{v^{k}-1}\right\}$ by applying a brute-force operation that sums up all of the combinations between the received possible plans. Furthermore, the aggregate plans from each child are merged by computing their summation. The resulting outcome plans are the aggregate plans $\mathbf{a}_{i}$ and $\hat{\mathbf{a}}_{i}$ of agent $i$.

- selection: Each agent $i$ chooses the best combinational plan $\mathbf{c}_{i}^{\prime}$. This selection is based on the stabilization objective that should be met. In practice, the selection for a stabilization objective is performed by one of the fitness functions $f_{\mathrm{MD}}\left(\mathbf{a}_{i}, \mathbf{C}_{i}\right)$ and $f_{\mathrm{RD}}\left(\hat{\mathbf{g}}, \hat{\mathbf{a}}_{i}, \mathbf{a}_{i}, \mathbf{C}_{i}\right)$. For each child $c$ of agent $i$, the selected plan $\mathbf{p}_{c}^{\prime}$ is derived from the selected combinational plan $\mathbf{c}_{i}^{\prime}$.

- update: The aggregate plan $\mathbf{a}_{i}$ of each agent $i$ is updated with the selected combinational plan $\mathbf{c}_{i}^{\prime}$ as $\mathbf{a}_{i}=\mathbf{a}_{i}+\mathbf{c}_{i}^{\prime}$. Furthermore, the aggregate plan $\hat{\mathbf{a}}_{i}$ is updated as $\hat{\mathbf{a}}_{i}=\hat{\mathbf{a}}_{i}+\hat{\mathbf{p}}_{i}^{\prime}$, where $\hat{\mathbf{p}}_{i}^{\prime}$ is the selected plan of agent $i$ at an earlier coordination phase.

- inform (children): Each agent $i$ sends the selected plan $\mathbf{p}_{c}^{\prime}$ to each of its child c.

- execution: Each agent $i$ configures the energy utilization of its device for a period of time as defined by the selected plan $\mathbf{p}_{i}^{\prime}$.

- broadcast: If agent $i$ is the root of the tree, it broadcasts the global plan $\mathbf{g}=\mathbf{a}_{i}$ to all of the agents of the tree. Agents forward the received broadcasted message from their parents to all of their children.

Figure 6.2 illustrates the concepts of a coordination step and phase in EPOS and the execution of the agent tasks between a parent and its children.

During a coordination step, children perform the tasks planning and in form (parent) (line 6, 13, 19 and 20 of Algorithm 6.1). The purpose of these actions is to let the parent, as aggregator, perform more informed decision-making about the plan that its children execute. The decision is more informed for two reasons: (i) the parent aggregates information from multiple agents, its children, and (ii) it receives information about the current selections in the running coordination phase, the aggregate plans. Next, the parent executes the aggregation task (line 2 of Algorithm 6.1) by computing its aggregate plans according to (6.1) and (6.2):

$$
\mathbf{a}_{i}=\sum_{c=0}^{k-1} \mathbf{a}_{c}
$$




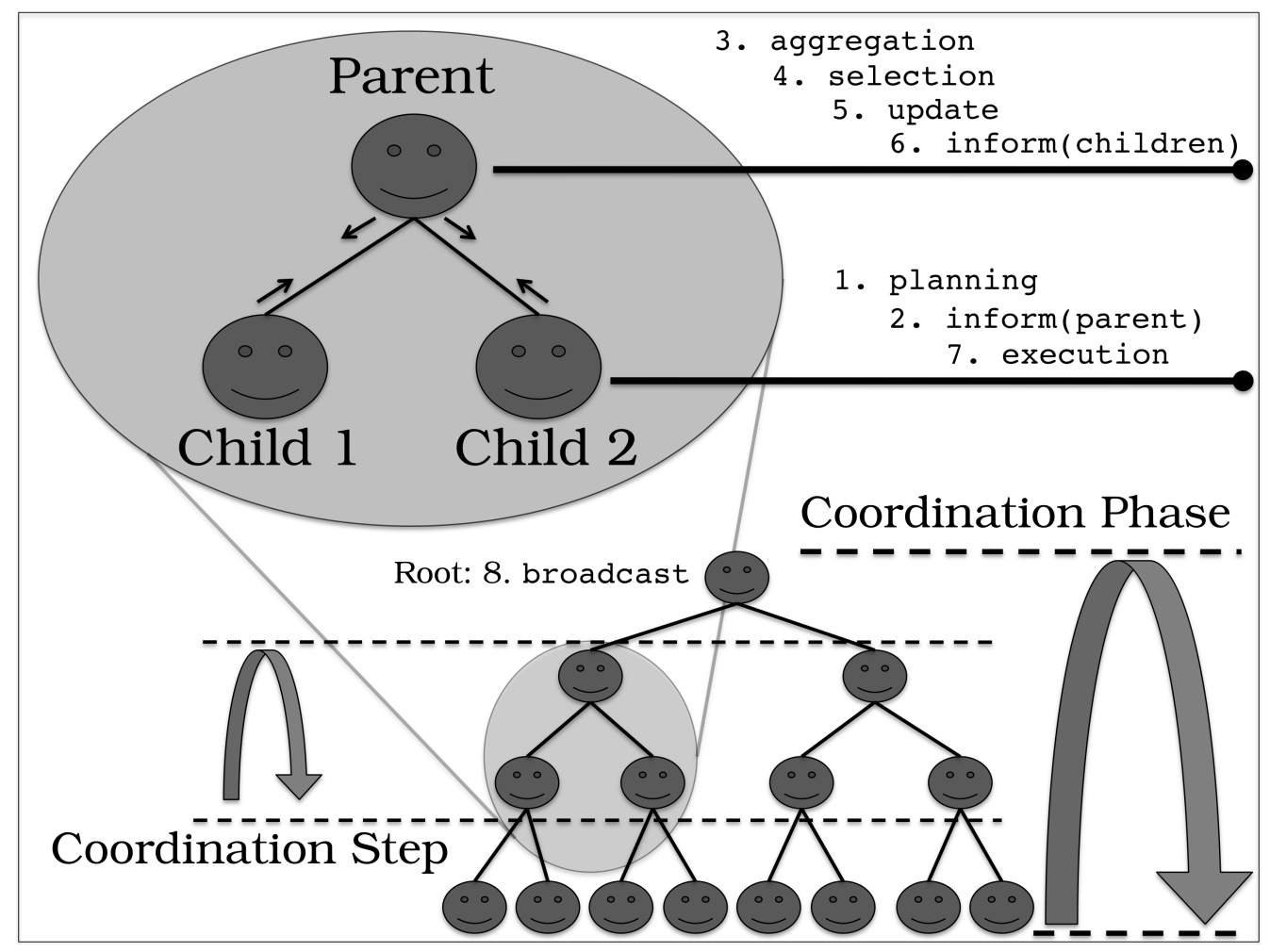

Figure 6.2: The coordination algorithm of EPOS.

$$
\hat{\mathbf{a}}_{i}=\sum_{c=0}^{k-1} \hat{\mathbf{a}}_{c}
$$

The aggregation task also concerns computation of the combinational plans $\mathbf{C}_{i}$ by an agent $i$ based on a brute-force operation performed between the received possible plans. For example, for a parent with two children each generating two possible plans, the total number of combinational plans is $\left|\mathbf{C}_{i}\right|=v^{k}=2^{2}=4$. These four combinational plans are computed in this case according to (6.3):

$$
\mathbf{C}_{i}=\left\{\mathbf{c}_{i}^{0}=\mathbf{p}_{0}^{0}+\mathbf{p}_{1}^{0}, \mathbf{c}_{i}^{1}=\mathbf{p}_{0}^{0}+\mathbf{p}_{1}^{1}, \mathbf{c}_{i}^{2}=\mathbf{p}_{0}^{1}+\mathbf{p}_{1}^{0}, \mathbf{c}_{i}^{3}=\mathbf{p}_{0}^{1}+\mathbf{p}_{1}^{1}\right\}
$$

The $\mathbf{p}_{c}^{j}$ is the $j$ possible plan of child $c$. Note that the complexity of the brute-force operation is controlled by the topological properties of the tree and specifically by the number of children per agent.

The next task executed is selection (line 3 of Algorithm 6.1). The purpose of this task is to select the best combinational plan according to one of the stabilization objectives defined at the beginning of this section: (i) minimum deviation and (ii) reversed deviation. Decision-making is based on knowledge adaptation that is the evaluation of deviation in all of the combinational plans if they are added (adapted) to aggregate plans. Deviation is computed based on the statistical standard deviation $\sigma$. The fitness function for the stabilization objective of minimum deviation is the following: 


$$
f_{\mathrm{MD}}\left(\mathbf{a}_{i}, \mathbf{C}_{i}\right)=\min _{j=0}^{\mathrm{v}^{k}-1} \sigma\left(\mathbf{a}_{i}+\mathbf{c}_{i}^{j}\right)
$$

The fitness function (6.4) computes the best combinational plan $\mathbf{c}_{i}^{\prime} \in \mathbf{C}_{i}$ that minimizes the standard deviation $\sigma$ if $\mathbf{c}_{i}^{\prime}$ is added to the aggregate plan $\mathbf{a}_{i}$.

For the stabilization objective of reversed deviation, assume an earlier coordination phase that results in an earlier global plan $\hat{\mathbf{g}}$. The current coordination phase should result in a recent global plan $\mathbf{g}$ that reverses the deviation of $\hat{\mathbf{g}}$, both devised by the same agents representing the same devices. For example, the reverse of deviation in the energy demand $\overline{\mathbf{g}}+x_{t}$ is the $\overline{\mathbf{g}}-x_{t}$, where $x_{t}$ is the deviation from the average total consumption $\overline{\mathbf{g}}$ at time $t$ of planning. The fitness function for reversed deviation is computed as follows:

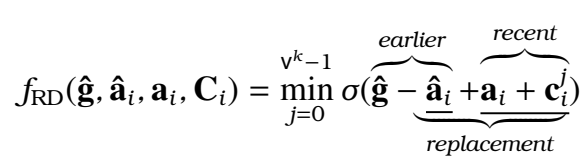

This computation shows that the earlier aggregate plan $\hat{\mathbf{a}}_{i}$ is replaced by the equivalent summation of the recent aggregate plan $\mathbf{a}_{i}$ and the combinational plan $\mathbf{c}_{i}^{j}$. Note that $\hat{\mathbf{a}}_{i} \equiv \mathbf{a}_{i}+\mathbf{c}_{i}^{j}$. This is a replacement (adaptation) to the earlier global plan $\hat{\mathbf{g}}$.

The concept behind this fitness function is the following: The average of the earlier global plan and recent global new plan should ideally result in a minimum (zero) deviation. This is because $\frac{\left(\overline{\mathbf{g}}+x_{t}\right)+\left(\overline{\mathbf{g}}-x_{t}\right)}{2}=\overline{\mathbf{g}}$. Choosing the combinational plan that contributes best on transforming $x_{t}$ to $-x_{t}$ results in a global plan with reversed deviation. The transformation is gradually and incrementally achieved during consecutive coordination steps.

Figure 6.3 visualizes the concept of the two fitness functions. Note that both stabilization objectives are achieved using the same algorithm execution and interactions.

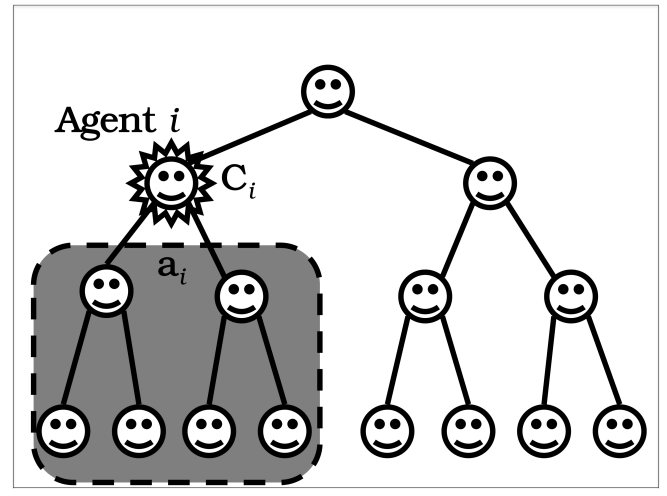

(a) Minimizing deviation.

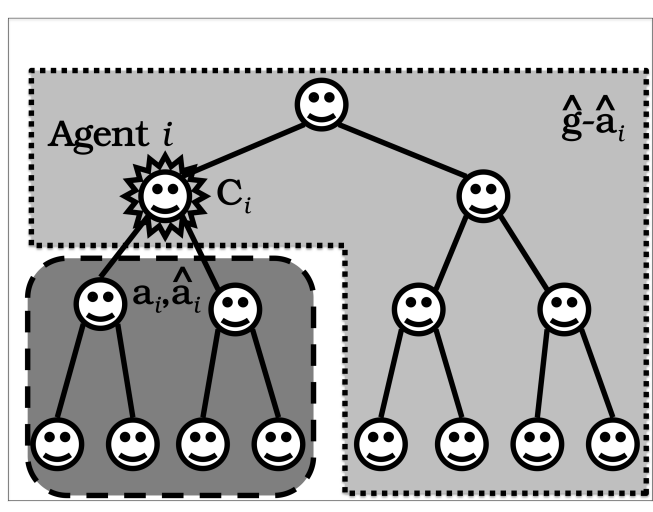

(b) Reversing deviation.

Figure 6.3: The planning information used by agents in decision-making.

After the execution of the selection task, the update and inform (children) tasks follow (line 4 and 5 of Algorithm 6.1). The parent updates its aggregates plan and the children execute their selected plan as defined by the parent agent (line 16 of 
Algorithm 6.1). This completes a coordination step for this agent. Note that if an agent is the root of the tree it performs an additional local coordination step. The root agent selects locally its executed plan (line 7-11 of Algorithm 6.1). In other words, the root agent performs two decision-making processes, one for its children and one for itself. In this case the possible plans are used as the combinational plans: $\mathbf{C}_{i}=\mathbf{P}_{i}$ for agent $i$ positioned as the root of the tree. Finally, a root agent $i$ completes the coordination phase by broadcasting the global plan $\mathbf{a}_{i}=\mathbf{g}$ to all agents in the tree. In this way, the agents start the next coordination phase and use this broadcasted global plan for future reverse of deviation if needed.

\subsubsection{Using the AETOS overlay service}

The use of a tree overlay network is a crucial design choice in EPOS. Communication efficiency and convergence are two of the reasons for this choice. A hierarchical structure supports an incremental coordination process with consecutive coordination steps in every level of a tree topology. Each coordination step contributes to stabilization of the cumulative aggregate plans computed during previous coordination steps. Note that aggregate plans can be computed accurately without counting duplicates due to the graph property of path uniqueness that tree topologies have. Furthermore, the organization of a tree overlay network controls the computational complexity of EPOS. More specifically, computation of the combinational plans using the brute-force operation becomes feasible by locally choosing the maximum number of children from which possible plans are received and combined. Therefore, the computational complexity is $O\left(\mathrm{v}^{k}\right)$ instead of $O\left(\mathrm{v}^{n}\right)$ to which centralized coordination is bound. Assuming that every agent generates an equal number of possible plans, a higher number of children results in locally more informed decisions at the cost of a higher computational complexity.

The AETOS overlay service, illustrated in Chapter 4, enables self-organization of trees used by EPOS. Instead of simply using a static tree that is affected by single peer failures, AETOS builds and maintains the tree topology during coordination. The planning period of EPOS can be used for tree organization and optimization. Moreover, AETOS is able to order a tree according to application criteria that potentially contribute to the stabilization of the energy consumption. For example, criteria such as the average energy consumption, or the deviation of possible plans can be used for ranking the agents with an impact on their positioning to the tree topology. Note that there is a high number of agents that are leaves and their aggregate plans containing zero values in contrast to only a few agents close to the root with aggregate plans that converge to the final global plans. Therefore, the position of an agent within a tree topology has a potential impact on the achieved stabilization.

The design approach of EPOS has a number of features in common with the DIAS overlay service illustrated in Chapter 5. EPOS performs aggregation in each and every coordination step. The concept of possible plans is similar to the concept of possible states that DIAS defines. Nevertheless, EPOS cannot simply replace AETOS by the DIAS overlay service. One reason is the fact that DIAS does not define a coordination mechanism but only performs aggregation. This means that agents cannot locally select their more stabilized possible plan as they are assumed to be equivalent. Selections are made collectively in respect to other possible plans generated by other agents. This is the reason why the agents of EPOS aggregate and combine the possible plans of their children. Even if local selections could be possible based on a global plan aggregated by DIAS, 
the problem of convergence remains unsolved and falls into other research problems such as the ones of reward and revenue sharing [Giannoccaro and Pontrandolfo, 2009]. DIAS assumes unstructured overlay networks and therefore the topology cannot intuitively instruct a coordination mechanism that enables EPOS to converge. For example, if an agent selects a possible plan that improves stabilization of a global plan, then this decision makes a second simultaneous selection by another agent biased.

\subsubsection{Experimental settings}

EPOS is implemented and evaluated in a customized simulation environment ${ }^{4}$. EPOS uses a degree-bounded, balanced and complete tree overlay network as described in Section 4.1.1. The tree overlay network of EPOS is built by the AETOS overlay service implemented in the Protopeer [Galuba et al., 2009] prototyping toolkit as illustrated in Section 4.7. The evaluation of EPOS focuses on the two stabilization objectives of minimizing and reversing deviation. EPOS is compared to a system in which the energy consumption is the intended one, without generating alternative energy plans ${ }^{5}$. EPOS is also compared to the optimum centralized coordination of complexity $O\left(v^{n}\right)$ in a smallscale network.

The experimental evaluation of EPOS addresses the following five questions:

Question 1. What is the degree of minimum deviation achieved with EPOS compared to the intended energy consumption and centralized coordination?

Question 2. What is the degree of reversed deviation achieved with EPOS?

Question 3. How does the number of possible plans influence the stabilization of EPOS?

Question 4. How does the local plan deviation influence the stabilization of EPOS?

Question 5. How does the organization of a tree overlay network influence the stabilization of EPOS?

The heterogeneity of thermostatically controlled devices organized in a tree overlay network is simulated based on the following: (i) the average consumption of a generic thermostatically controlled appliance, (ii) the deviation of this average consumption that represents different types of thermostatic devices (refrigerator, water heater etc.) and (iii) the total number of these types. A number of average consumption seed values are randomly generated for every type of device. The seed values belong to the range defined by the deviation of average device consumption. The consumption of a specific device type varies as well, however, this variation is assumed lower in general compared to the deviation of average consumption among different types of devices, e.g., a refrigerator and a water heater.

Moreover, each agent in a tree overlay network, regardless of which type of device it represents, generates a fixed number of possible plans over a fixed time period. Generation of plans is simulated as follows: For each final average consumption of each device, a random sample is generated with size equal to the number of energy values of the plan. The values are estimated between a (plus/minus) percentage range

\footnotetext{
${ }^{4}$ The source code of the simulator and the implementation of EPOS is available by the author of this thesis on request.

${ }^{5}$ This approach is implemented by configuring agents of EPOS to generate a single possible plan.
} 
of plan deviation from the average consumption value of the plan. For example, a $20 \%$ plan deviation from the average consumption value of 10 units, results in deriving random values in the range $[8,12]$.

Finally, user consumption profiles are simulated. Each device has a 'high', a 'medium' and a 'low' consumption profile. A coefficient for each consumption profile multiplies or divides respectively the values of the possible plans. The profiles change cyclically in every coordination phase and are initially attributed randomly to devices. This means that each individual device may start with any of the high, medium or low consumption profiles and its selections follow the same cyclical row in each coordination phase.

Note that an agent could have the option to locally select the most stabilized plan without adaptation to the aggregate plans as the fitness functions define. However, possible plans are generated with equivalent deviation in the experimental settings as shown in this section. Therefore, the stabilization achieved with the locally optimum selected plans is equivalent with the intended energy consumption implemented by generating a single possible plan. Experimental results confirm this intuition.

Four experimental settings are defined for the evaluation of EPOS. Table 6.1 outlines the parameterization for each experimental setting. SETting A is a small-scale environment of $n=15$ agents for comparing EPOS with optimum centralized coordination. SETting B is used as an illustrative environment for the evaluation of the minimum deviation fitness function. It is also used for evaluating the same fitness function under a varying number of possible plans. SETting C does not use consumption profiles. The deviation of the generated possible plans varies in the performed experiments. SETTING $\mathrm{C}$ is used to shows if local deviation affects the global deviation. Finally, SETTING D varies the number of children over a series of experiments to evaluate how tree organization affects the stabilization of EPOS. It does not also use consumption profiles and keeps the number of possible plans equal to $v=3$.

Table 6.1: The experimental settings used for the evaluation of EPOS.

\begin{tabular}{|l|c|c|c|c|}
\cline { 2 - 5 } \multicolumn{1}{c|}{} & SetTing A & SetTing B & Setting C & Setting D \\
\hline Number of agents & 15 & 3280 & 3280 & 3280 \\
\hline Number of children/agent & 2 & 3 & 3 & $2-5$ \\
\hline Number of possible plans & 2 & $2-7$ & 5 & 3 \\
\hline Number of values/plan & 10 & 10 & 10 & 10 \\
\hline Average device consumption & 0.5 & 0.5 & 0.5 & 0.5 \\
\hline Number of device types & 3 & 3 & 3 & 3 \\
\hline Average consumption deviation/type & 0.35 & 0.35 & 0.35 & 0.35 \\
\hline Average consumption deviation/type/device & 0.035 & 0.035 & 0.035 & 0.035 \\
\hline Plan deviation & $90 \%$ & $90 \%$ & $10 \%-90 \%$ & $50 \%$ \\
\hline Number of consumption profiles & 3 & 3 & 1 & 1 \\
\hline Consumption profile coefficient & 2 & 2 & 1 & 1 \\
\hline
\end{tabular}

Note that the simulated unit for energy consumption is the same in all of the experimental settings. Simulations run for $t(E P O S)=100$ coordination phases and the illustrated results are averaged over this running period. 


\subsubsection{Evaluation results}

The objective of minimizing deviation is evaluated by computing the standard deviation of the global plans at the end of each coordination phase. Figure 6.4 compares EPOS with the optimum centralized coordination and the intended energy consumption using SETTING A.

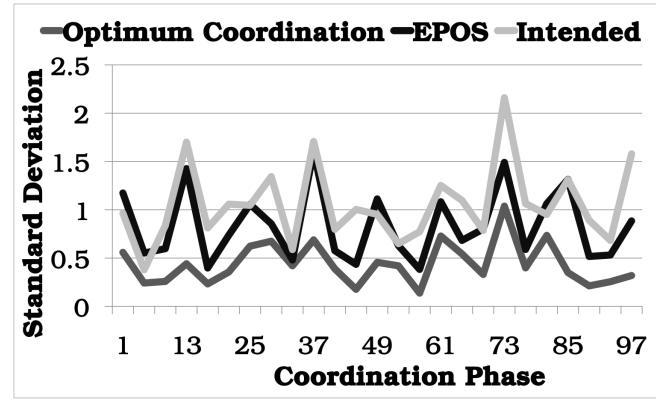

Figure 6.4: The minimization of deviation achieved with centralized coordination, EPOS and the intended energy consumption in SETTING A.

The results collected indicate that the stabilization achieved with EPOS lies between the stabilization of the intended energy consumption and optimal centralized coordination. The average standard deviation for 100 coordination phases in the centralized coordination is 0.42 , whereas, for EPOS and the intended energy consumption the standard deviation is 0.79 and 1.08 respectively.

Figure 6.5 illustrates an example of three groups of global plans with different consumption profiles. Each group shows the global plan of EPOS and the one of the intended energy consumption. The data are collected using setTing B with 5 possible plans per agent.

In each coordination phase, or every 10 time intervals, the energy consumption changes due to the local consumption profiles. The deviation decreases $78.71 \%, 36.54 \%$ and $73.46 \%$ by using EPOS compared to the intended energy consumption. Note that EPOS does not aim to stabilize the energy consumption between different consumption profiles. The scale of the global energy consumption unavoidably changes at different times periods and, therefore, EPOS focuses on the decrease of positive and negative energy peaks within each global plan.

The objective of reversed deviation is evaluated by computing the correlation coefficient [Rodgers and Nicewander, 1988] of the global plans that are reversed during runtime. Ideally, the optimum reversing between two global plans corresponds to a correlation coefficient that is equal to -1 . Figure 6.6 a illustrates the values of the correlation coefficient during runtime. Figure $6.6 \mathrm{~b}$ depicts the reversing effect in two global plans. The data are collected using SETTING $\mathrm{C}$ with $70 \%$ plan deviation.

Figure 6.6a shows that there is always a negative correlation between two global plans on which the fitness function of reversed deviation is applied. This means that EPOS reacts always positively as shown in these experimental settings. The average value of the correlation coefficient is -0.8 in this case. In addition, Figure $6.6 \mathrm{~b}$ illustrates the reversing effect in two global plans. The recent global plan converges to a mirroring version of the earlier global plan. The average of these two plans is also drawn to depict 


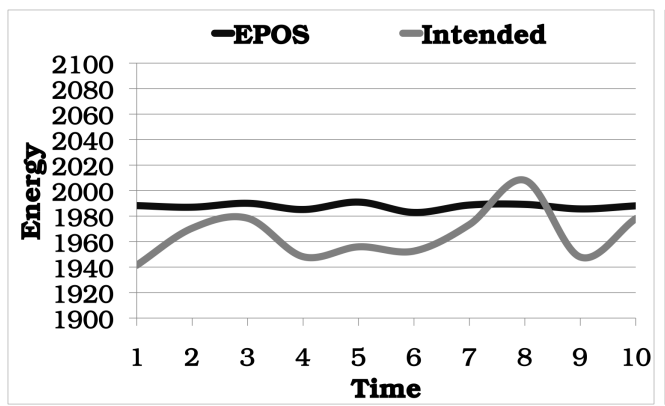

(a) First coordination phase

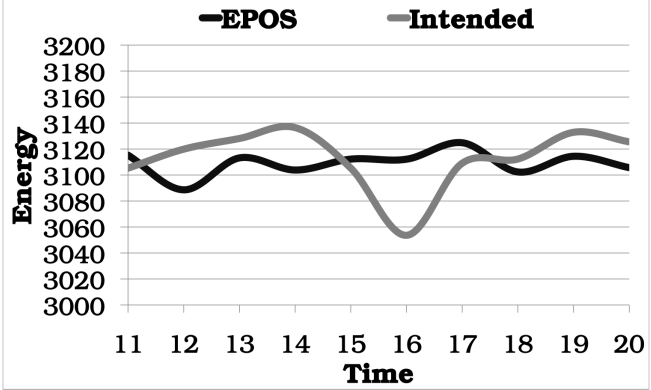

(b) Second coordination phase

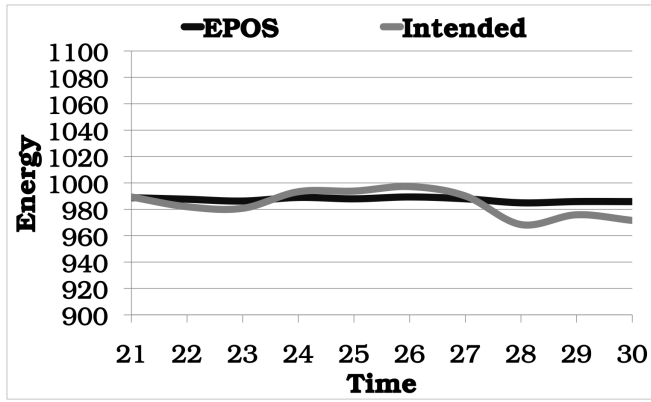

(c) Third coordination phase

Figure 6.5: The global plans of three consecutive coordination phases during which three consumption profiles are utilized. Results are computed using SETTING B.

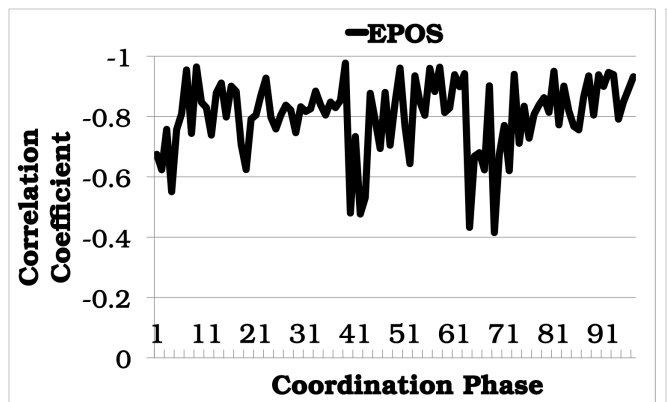

(a) The correlation coefficient achieved during runtime.

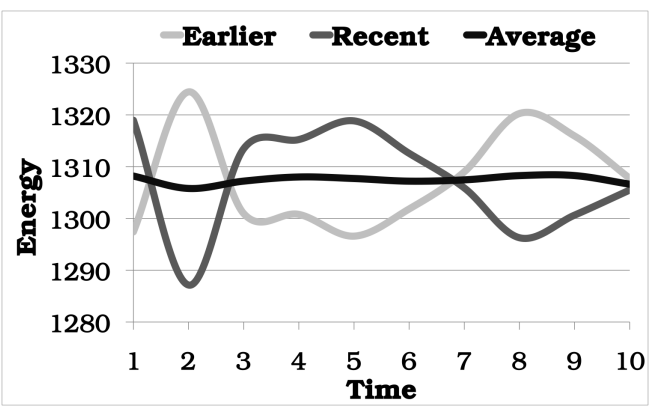

(b) An example of reversing deviation between two global plans. Their average is depicted as well.

Figure 6.6: The reverse of deviation achieved with EPOS in SETTING C.

the effect of the nearly flat average energy consumption.

The number of possible plans is one of the critical local constraints of the stabilization problem. The purpose of the following experiments is to examine how the number of local options that agents have affects the global stabilization achieved. For this reason, multiple experiments have been performed in SETTING B by varying the number of possible plans v. Figure 6.7 illustrates the results for the two stabilization objectives. 


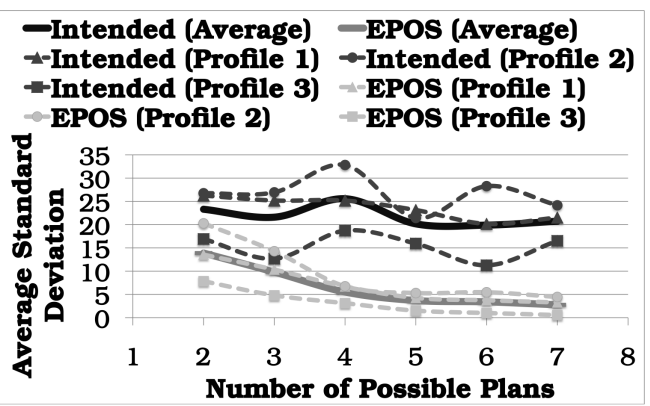

(a) The average standard deviation of EPOS and intended energy consumption for the stabilization objective of minimum deviation.

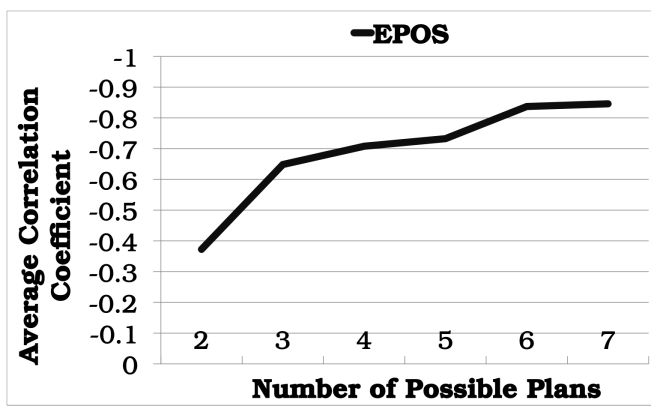

(b) The average correlation coefficient of EPOS for the stabilization objective of reversed deviation.

Figure 6.7: The effect of varying the number of possible plans $v$ that agents generate in EPOS. The data are collected using SETTING B.

Figure 6.7a illustrates the average standard deviation of the global plans during runtime in EPOS and the intended energy consumption for the stabilization objective of minimum deviation. The increase in the number of possible plans $v$ influences the stabilization of EPOS positively as the values of the standard deviation decrease. For 2 possible plans, the difference in the decrease of standard deviation is 9.41 between EPOS and the intended energy consumption. In contrast, for 7 possible plans it almost doubles to 17.96, denoting almost double improvement. The reason for this positive influence is the increased number of options from which agents can choose, or the higher degree of freedom that agents have, resulting in a higher potential for minimum deviation. The number of possible plans also influences the reversing effect as Figure $6.7 \mathrm{~b}$ shows. The values of the correlation coefficient decrease from -0.37 to -0.85 as the number of possible plans $v$ increases in the range of $[2,7]$. Similar to the case of minimizing deviation, the agents have more options from which they make selections and therefore, a higher potential to reverse an earlier global plan effectively.

Local deviation in the possible plans affects the deviation of the global plans in EPOS and the intended energy consumption. In other words, higher stabilization is achieved if the local plans have a higher variation from their average. In the experiments below, the percentage of deviation from the average varies between $10 \%$ and $90 \%$ respectively as defined by SETTing $\mathrm{C}$ that is adopted for these experiments. The results of the two stabilization objectives are outlined in Figure 6.8.

Figure 6.8a shows a clear linear relationship between the deviation of the possible plans from their average and the deviation in the final global plans. The average standard deviation increases from 0.76 to 6.63 for EPOS and from 1.50 to 13.42 for the intended energy consumption. Note that the improvement in the stabilization between EPOS and the intended energy consumption is nearly 50\% in SETTING C. In contrast, Figure $6.8 \mathrm{~b}$ shows different results. In this case, as the local deviation of the possible plans from the average increases, the correlation coefficient of the reversed global plans decreases from -0.75 to -0.84 . These results show that a higher deviation in the possible plans results in a more effective reversing effect. These results indicate that without deviation in the possible plans, the system is unable to converge. The stabilization objective of reversed deviation requires some operational flexibility to reverse a global 


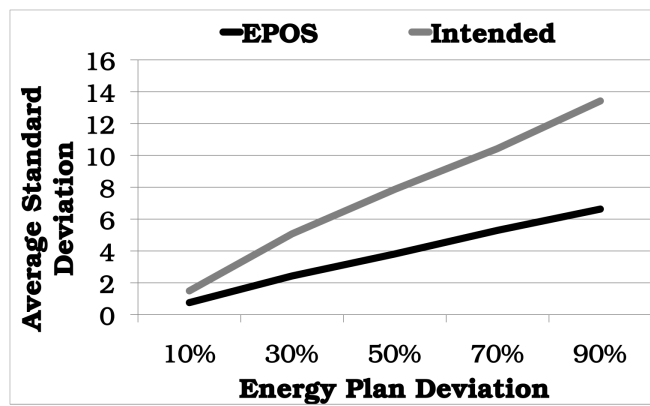

(a) The average standard deviation in EPOS and the intended energy consumption for the stabilization objective of minimum deviation.

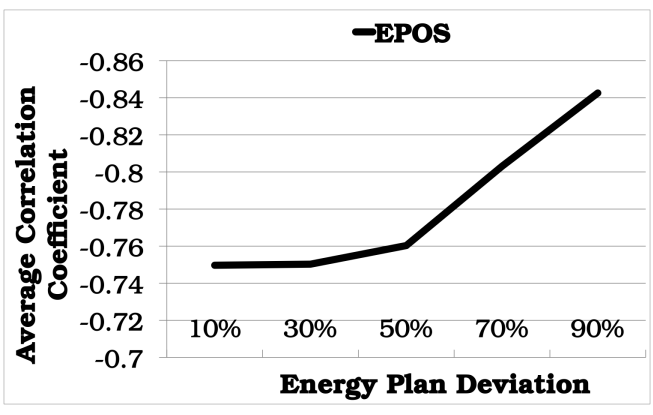

(b) The average correlation coefficient in EPOS for the stabilization objective of reversed deviation.

Figure 6.8: The effect of varying the percentage of plan deviation from the average in EPOS. The data are collected using seTting C.

plan. This flexibility is explored via the local deviation of the possible plans.

The stabilization scheme of EPOS is based on a tree overlay network. The influence of tree organization in the effectiveness of EPOS is studied. Although there are various topological aspects related with the tree organization, this chapter only investigates the variation of the maximum number of children $k$ that is used for the AETOS overlay service. The parameter $k$ is varied from 2 to 5 . A higher number of children is not adopted as storing and processing complexity increases exponentially.

By varying the number of children $k$ from 2 to 5 , the topology of the tree overlays changes as follows: for 3280 agents, the number of levels $h$ in the tree is $h=12$, $h=8, h=7$ and $h=6$ respectively. The number of leaves in each topology is 1233 , 2187, 1915 and 2499 respectively. These numbers mean that the tree organization changes from being 'long' and 'thin' to 'short' and 'fat'. The fact that the tree structure changes to 'short' and 'fat' also indicates that the overlay network converges towards a star topology with a single aggregator that is able to perform a centralized optimum coordination. Figure 6.9 illustrates the results of the two stabilization objectives using SETTING D.

Figure 6.9a shows that the increase of the number of children $k$ results in a decrease of the average standard deviation by 1.89 in EPOS. This value is relatively low, therefore, definite conclusions cannot be reached about how positively the increase in the number of children influences the stabilization objective of minimum deviation. Similarly, Figure $6.9 \mathrm{~b}$ cannot provide a clear indication whether the number of children influences the stabilization objective of reversed deviation in EPOS. In this case, there is again an improvement, but the difference in the correlation coefficient is 0.07 that is extremely low.

Despite the challenge to clearly explain these results, the explanation that this chapter provides is based on the fact that there is a trade-off between adaptation and optimality in decision-making. This means that when the number of children increases, the agents compute a higher number of combinations and their decisions are more informed. However, the tree overlay network has fewer levels and therefore fewer adaptations are performed. Note that in every coordination step, the aggregate plans are used for adapting the combinational plans. Every coordination step corresponds to a 


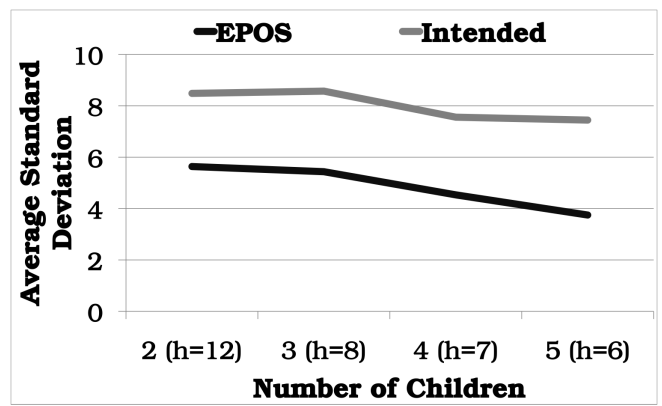

(a) The average standard deviation in EPOS and the intended energy consumption for the stabilization objective of minimum deviation.

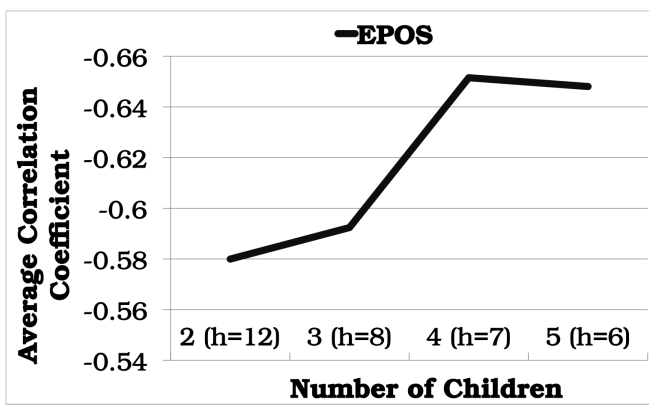

(b) The average correlation coefficient in EPOS for the stabilization objective of reversed deviation.

Figure 6.9: The effect of varying the number of children $k$ per agent in EPOS. The data are collected using SETTING D.

level in the tree overlay network. This explains why adaptations are lower in number if the number of levels in the tree is lower as well.

\subsubsection{Evaluation summary}

The results collected for the evaluation of EPOS using the four experimental settings of Table 6.1 provide the following answers to the questions set in Section 6.3.3:

Question 1. What is the degree of minimum deviation achieved with EPOS compared to the intended energy consumption and centralized coordination?

EPOS always achieves a higher stabilization than the intended energy consumption. The results collected from SETTING B show that deviation decreases in the range of $36.54 \%-78.71 \%$ and the decrease is $50 \%$ in sETTING $C$. The experimental setting with the small-scale network of agents indicates stabilization for EPOS (0.79) between centralized coordination (0.42) and the intended energy consumption (1.08).

Question 2. What is the degree of reversed deviation achieved with EPOS?

EPOS always achieves a negative correlation that ranges from -0.37 to -0.85 in the experimental settings of Table 6.1. The average result of the two global plans corresponds to a 'flat' stabilized plan with its deviation approaching zero.

Question 3. How does the number of possible plans influence the stabilization of EPOS?

The increase in the number of possible plans $v$ increases the stabilization achieved in both fitness functions. In the stabilization objective of minimum deviation, the improvement between EPOS and the intended energy consumption almost doubles. In the stabilization objective of reversed deviation, the negative correlation also increases and reaches the value of -0.85 in the results collected using SETTING B.

Question 4. How does the local plan deviation influence the stabilization of EPOS? 
The deviation of the possible plans influences the deviation of the global plans as the experiments using SETTING C show. The stabilization for minimum deviation deteriorates linearly as deviation in the possible plans increases. In contrast, the stabilization achieved for the objective of reversed deviation benefits from the local deviation of the possible plans. The correlation coefficient decreases from -0.75 to -0.84 .

Question 5. How does the organization of a tree overlay network influence the stabilization of EPOS?

The experimental evaluation could not provide a clear answer to this question. Stabilization is influenced positively if the number of children increases. However, the influence is negligible. This issue must be further investigated in regards to other topological properties such as the ordering of the tree performed by AETOS.

\subsection{Adaptive Load Management by Aggregation}

EPOS cannot address a situation in which consumers need to adapt their consumption behavior and reduce or even increase their energy consumption as a response to (i) failures in the power supply or (ii) micro-generation that exceeds current demand respectively. In these cases, one option is that consumers need to sacrifice either a level of comfort or economy for a period of time as a contribution to meet the available power supply and prevent system blackouts. The adjustments required concern not only the reduction of energy consumption but also the increase that is needed to meet the varying availability of renewable energy resources. Note that Paulus and Borggrefe [2011] estimate an accumulation of positive and negative balancing power of 33\% and $41 \%$ in demand by 2030 in Germany, due to the integration of wind generation.

The problem of controlling the actual energy consumption of consumers is challenging and is influenced by various socio-technical and economic factors. For example, designing incentives to influence energy consumption by sacrificing a level of comfort or economy is a crucial aspect as discussed in Section 6.1.1. This section focuses on the feasibility of load management for enabling a level of energy reduction or increase in the current reality and practice of Smart Power Grids.

Consumers require knowledge and awareness about the current energy consumption and available supply capacity to adapt their energy consumption. Aggregation can provide this knowledge and awareness and, therefore, it is a crucial and core operation required by load management mechanisms. This section introduces the ALMA mechanism: Adaptive Load Management by Aggregation. ALMA is an application of the DIAS overlay service that performs dynamic decentralized aggregation as illustrated in Chapter 5. On the one hand utility companies disseminate the available supply capacity to consumers without the need to aggregate their individual energy demand in a centralized fashion. On the other hand, software agents installed in household devices of consumers disseminate and collect information with each other about their current demand. Based on the aggregate demand in the network, agents adapt their local demand to meet the available supply capacity.

Figure 6.10 illustrates an overview of ALMA. Adaptation of local demand is performed based on the dynamic selection between a number of predefined demand options. These options are the possible states of DIAS and the chosen demand is the selected state. The possible states of DIAS represent incremental levels of comfort and economy that user 
may experience when a certain possible state (demand) is selected. However, note that all demand options are operationally possible for agents to realize. In addition, consumers accept and approve all of these options. Decision-making expresses their preference level between comfort and economy and selections can be performed (i) manually by the consumers themselves via a user interface or (ii) by an agent strategy that consumers approve and reconfigure. For example, consumers have the option to configure the agents of DIAS to select possibles state that correspond to $80 \%$ comfort during a day. Moreover, a consumer may desire an economical consumption that should not exceed a certain number of hours during a day. The definition of possible states and the actual decision-making scheme of selected states should be incentivized and negotiated by utility companies with consumers via a demand/response program.

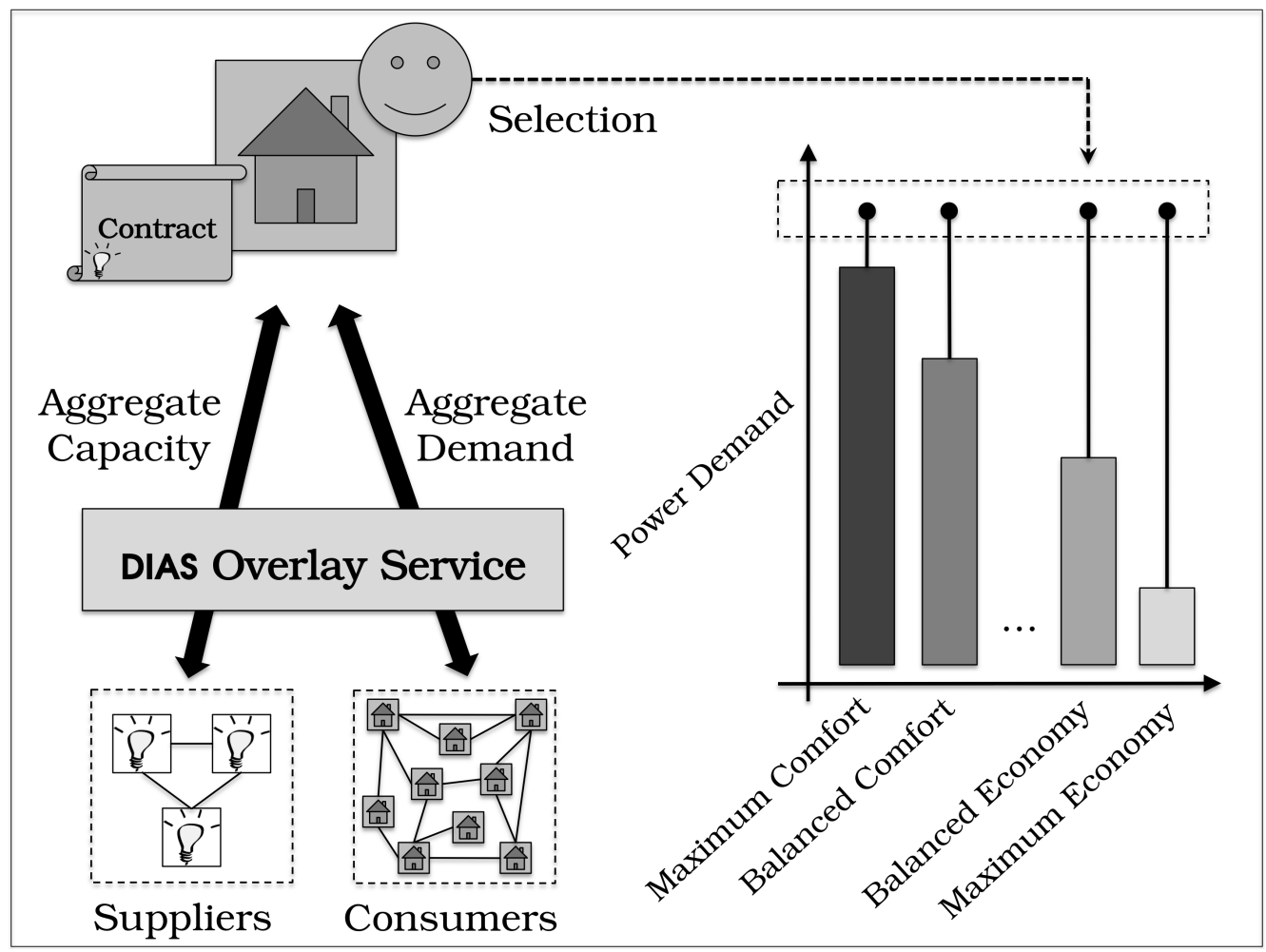

Figure 6.10: Demand-side energy management using ALMA. Consumers select between different demand options representing levels of comfort and economy in their energy consumption. The selections are based on the aggregate demand and capacity in the Smart Power Grid.

This section evaluates the feasibility of ALMA in an operational Smart Power Grid. More specifically, this section follows an analytical approach to validate the following hypothesis:

Hypothesis. Adjustments of aggregate energy consumption can be achieved with possible demand options of local energy consumption, representing a wide range of comfort and economy levels, that can be pre-defined and dynamically selected by incentivized consumers. 
This hypothesis is validated within the context of a well studied and state of the art demonstration project that involves real customers, utility companies and other stakeholders under realistic settings that shape and underpin the future Smart Power Grids: The Olympic Peninsula Smart Grid Demonstration Project [Hammerstrom, 2007]. The rest of this section provides an overview of this project and the validation approach followed. The analytical results computed using the actual data of the Olympic Peninsula project confirm the above hypothesis.

\subsubsection{The Olympic Peninsula Project}

The Olympic Peninsula Project, illustrated in the report of Hammerstrom [2007], is a Smart Power Grid demonstration project funded by the U.S. Department of Energy and led by the Pacific Northwest National Laboratory (PNNL). A wide range of stakeholders ${ }^{6}$ are involved such as regional utility companies, balancing authorities etc. Within this project, consumers play an active role in managing the Smart Power Grid by adjusting their individual energy use based on price signals. These signals are exchanged between consumers and utility companies, forming a two-way bidding market. Interactions are supported by novel communication technologies such as the Internet-Scale Control System (iCS) [Ambrosio et al., 2011]. The advantages of the approach followed are (i) improvement of reliability in the Smart Power Grid, (ii) reductions of consumers' bills, (iii) minimization of the future infrastructure investments and (iv) higher integration of renewable energy resources. The results collected in the period between March 2006 and March 2007 show that a 15\% peak reduction can be achieved during a year and consumers can lower their energy bills by $10 \%$. Furthermore, $\$ 70$ billions can be saved in a 20 year period by avoiding infrastructure changes in generation, transmission and distribution systems that are required to meet the increasing demand.

Three types of controllable assets are involved in the project: (i) Two backup diesel generators, (ii) five water-pumping stations and (iii) 112 households. For illustration purposes, this section focuses on the controllable assets of households. Engineering details about the control of generators and water-pumping stations are provided in the project report [Hammerstrom, 2007]. Despite the fact that the households are regionally distributed and connected to different feeders ${ }^{7}$, the project positions consumers within a single virtual feeder under the control of a dashboard software platform managed by grid operators. The capacity of this virtual feeder is varied at different periods during the project year to evaluate the consumer behavior under different capacity constraints.

Three types of contracts are assigned to consumers: (i) FIXED, (ii) TIME OF USE and (iii) REAL TIME PRICING. The FIXED contract does not involve changes in the electricity prices regardless of the amount and times of consumption. This group is the most inflexible to react to price incentives. The TIME OF USE contract involves three electricity price schemes, the 'off-peak', 'on-peak' and 'critical peak'. The price of electricity increases for each scheme respectively. This group has the option to configure home automation software to match certain comfort and economy settings in each price scheme. Finally, the REAL TIME PRICING contract varies the electricity price every five minutes. This contract

\footnotetext{
${ }^{6}$ Some of the stakeholders involved are the Bonneville Power Administration, PacifiCorp, Portland General Electric, the City of Port Angeles and Clallam County Public Utility District \#1. Industrial collaborators include Invensys Controls, Whirlpool Corporation and IBM Thomas J. Watson Research Center.

${ }^{7}$ Feeders are circuits of the distribution system that connect substations with end-consumers. They run along streets or underground and power the distribution transformers at or near the consumer premises.
} 
type is the most flexible as it provides to consumers the option to continuously adjust their level of comfort and economy and, therefore, achieve the highest bill savings. Finally, there is a fourth group of consumers, the conTROL group, that is used for the evaluation of the demand/response program. Consumption information is collected from this group as well, however, the difference is that consumers do not have any contract within the context of the project.

Load-control modules are installed in HVAC systems, water heaters and cloth dryers of consumers. These modules communicate wirelessly with a home gateway from which communication with a centralized PNNL shadow market is performed every five minutes. This communication is two-way and concerns a bidding price and a clearing price for this period of five minutes. The bidding price represents the current demand based on history information about the clearing prices and the preference of consumer about the level of comfort and economy. The clearing price for a certain five-minutes period of time is the marginal ${ }^{8}$ price at which the aggregate load curve and demand curve intersect. The exact computation of both bidding and clearing prices is out of the scope of this chapter and is illustrated in detail by Hammerstrom [2007].

Consumers adjust their power demand by making selections about (i) the occupancy mode and (ii) the heating/cooling mode. These modes are referred to in this this chapter as consumption modes. An occupancy mode represents a temperature configuration related with a certain state of a consumer, e.g., away, sleep etc. Eight possible occupancy modes can be pre-programmed by consumers. Technically, an occupancy mode is configured by a temperature setpoint. However, consumers with a REAL TIME PRICING contract additionally select for each of their controllable devices a temperature range between a number of pre-defined temperature ranges. Each range is defined by a maximum and minimum temperature in relation to the configured temperature setpoint. Within this range, energy saving can be achieved. For examples, consumers of HVAC systems with a REAL TIME PRICING contract select between five temperature ranges that represent incremental levels of comfort and economy as illustrated in Table 3.4 of the project report [Hammerstrom, 2007]. Note that the actual temperature ranges are system parameters and cannot be modified by consumers. Finally, the heating/cooling mode concerns the selection between three operational modes of HVAC systems: heating, cooling and automatic. Note that each occupancy mode concerns temperature configurations for both heating and cooling.

\subsubsection{Validation approach}

Validation of the hypothesis set in this section is based on analysis of power demand from the Olympic Peninsula Smart Grid Demonstration Project [Hammerstrom, 2007]. The project data ${ }^{9}$ used concern the demand bids of the households consumers made every five minutes during the project year. Appendix $\mathrm{C}$ outlines the data of the project database used for validation. For illustration purposes, assume a function $f(t, j, i, o)$ that computes the power demand using the project data. $f(t, j, i, o)$ is computed based on the following information:

- $t \in[1,288]$ : The five minutes period of a project day during which a bid is sent.

\footnotetext{
${ }^{8}$ In the context of the Olympic Peninsula Project, the marginal price is the change in the total price as a result of a unit change in demand.

${ }^{9}$ Available at: https://svn.pnl.gov/olypen (last accessed: May 2012)
} 
The total number of bids during a day is $288=60$ minutes $* 24$ hours $/ 5$ minutes.

- $j \in[1,365]:$ The project day in which the bid $t$ is sent. The total number of projects days are 365 .

- $i \in[1,112]$ : The consumer who sends the bid $t$. The total number of residential consumers is 112 .

- $o \in\left\{\begin{array}{ll}{[0,7]} & \text { for occupancy modes } \\ {[0,2]} & \text { for heating/cooling modes } \\ {[0,23]} & \text { for their combination }\end{array}\right\}$ :

The consumption mode selected when the bid $t$ is sent. The selection is made from the 8 occupancy modes, the 3 heating/cooling modes or their $8 * 3=24$ combinations.

The goal of validation is to show if the power demand aggregated from the total consumers is influenced by alternative selections of consumption modes. An adjustment of the aggregated power demand may come as a result of selecting different consumption modes compared to the actual selections. Technically, (i) aggregation of information about power demand and (ii) actual selections of consumption modes are performed using the DIAS overlay service. Maximum and minimum adjusted power demand are computed by analyzing the average level of power demand for each selected consumption mode. The analysis performed concerns the computation of the following information:

- $D_{j}^{o}$ : The cumulative power demand of the total consumers that select the consumption mode $o$ during the day $j$ of the project.

- $N_{j}^{o}$ : The number of samples (bids sent every five minutes) based on which the cumulative power demand $D_{j}^{o}$ is computed.

- $N_{j}$ : The total number of samples (bids sent every five minutes) for the total consumers during the day $j$ of the project.

Note that $N_{j}$ counts the bids regardless of the selected consumption mode. The cumulative power demand $D_{j}^{o}$ during a day $j$ of the project with the consumption mode $o$ selected is computed as follows:

$$
D_{j}^{o}=\sum_{i=1}^{112} \sum_{t=1}^{288} f(t, j, i, o)
$$

Note that bids with zero power demand are excluded from the data analysis as they bias the computed results. These bids are treated as if they contain no information. Based on $D_{j}^{o}, N_{j}^{o}$ and $N_{j}$, the adjusted cumulative power demand $\hat{D}_{j}^{o}$, when consumption mode $o$ is selected during the day $j$ of the project, is computed as follows:

$$
\hat{D}_{j}^{o}=\frac{D_{j}^{o}}{N_{j}^{o}} N_{j}
$$

Based on the above, the maximum and minimum adjusted power demand for a day $j$ of the project is computed as follows:

$$
\hat{D}_{j}^{\mathrm{MIN}}=\min _{o=0}^{x} \hat{D}_{j}^{o}
$$




$$
\hat{D}_{j}^{\mathrm{MAx}}=\max _{o=0}^{x} \hat{D}_{j}^{o}
$$

For $x= \begin{cases}7 & \text { for occupancy modes } \\ 2 & \text { for heating/cooling modes } \\ 23 & \text { for their combination }\end{cases}$

The goal of validation is to compare both maximum and minimum adjusted power demand $\hat{D}_{j}^{\text {MAx }}, \hat{D}_{j}^{\text {MIN }} \forall j \in[1,365]$ with the actual power demand $D_{j}$ of the raw data during the project year. This comparison indicates if the actual power demand can be adjusted by making different selections of consumption modes using the DIAS overlay service supporting a dynamic and decentralized aggregation. Confirmation of the hypothesis is based on this comparison.

\subsubsection{Analytical results}

Figure 6.11 compares the actual power demand $D_{j} \forall j \in[1,365]$ of the raw data with the minimum $\hat{D}_{j}^{\mathrm{MIN}}$ and maximum $\hat{D}_{j}^{\mathrm{MAx}}$ power demand computed. If adjustments are performed via selections of occupancy modes, the minimum and maximum adjusted power demand computed on average during the project year is $6362.5 \mathrm{KW}$ and $23937.2 \mathrm{KW}$ respectively when the actual power demand is $11844.7 \mathrm{KW}$. In contrast, these adjustments are significantly lower in heating/cooling modes. The minimum and maximum adjusted power demand computed on average during the project year is $7131.6 \mathrm{KW}$ and $12324.9 \mathrm{KW}$ respectively. Finally, the combined occupancy and heating/cooling modes provide the highest adjustments of power demand. The minimum and maximum adjusted power demand computed on average during the project year is $4793.1 \mathrm{KW}$ and 25048.0 KW respectively.

One aspect investigated is the comparison of the actual consumption modes with regards to the contribution in the adjustment of power demand. In the case of occupancy modes ' 1 ', ' 2 ', the highest decrease of power demand by $1888.3 \mathrm{KW}$ and $3236.6 \mathrm{KW}$ on average per day respectively is observed. The selections of occupancy modes '4' and '5' have the highest increase of power demand, 3999.0 KW and 6982.2 KW on average per day respectively. However, note that no conclusions can be reached about the effect of certain occupancy modes as their semantic is only known to consumers who define it by choosing their temperature configurations. In contrast to the occupancy modes, the heating/cooling modes have a clearer impact on the adjustment of power demand. The selection of cooling has the highest decrease of power demand that reaches 3920.5 $\mathrm{KW}$ on average per day, whereas, the highest increase of power demand is computed by the selections of heating modes with $195.2 \mathrm{KW}$ on average per day. The potential of a decrease in power demand using cooling instead of heating is expected given the fact of extreme cold winter conditions in the Olympic Peninsula [Hammerstrom, 2007]. Finally, the combination of occupancy mode ' 1 ' with cooling and occupancy mode ' 2 ' with heating results in the highest decrease of power demand by $3422.5 \mathrm{KW}$ and 2658.5 KW on average per day respectively. The highest increase of power demand is 6162.5 $\mathrm{KW}$ and $7314.5 \mathrm{KW}$ on average per day respectively for the combination of occupancy mode ' 4 ' with heating and the occupancy mode ' 5 ' with heating.

The influence of the contract type assigned to each consumer is investigated as well. The actual, minimum adjusted and maximum adjusted power demand of different 


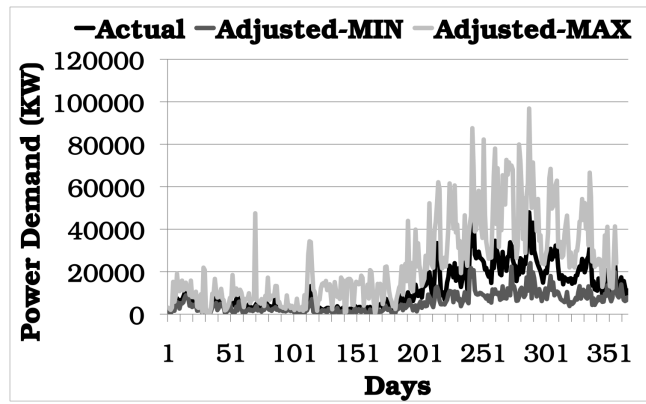

(a) Occupancy modes.

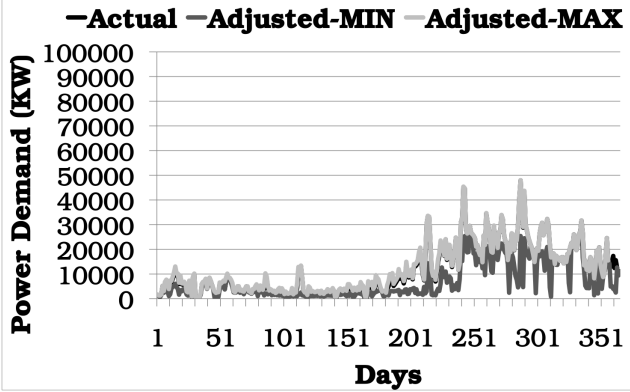

(b) Heating/cooling modes.

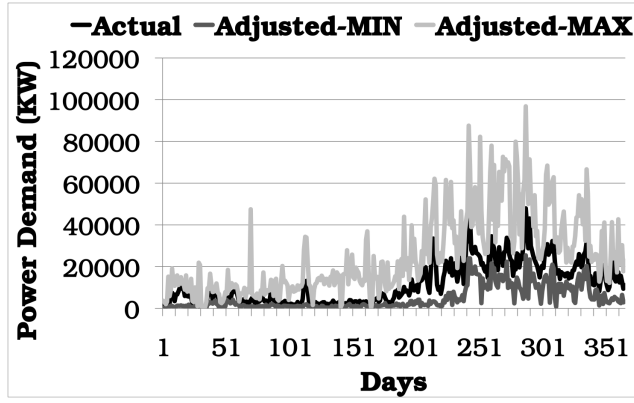

(c) Combining occupancy and heating/cooling modes.

Figure 6.11: The actual, minimum and maximum adjusted power demand during the project year by performing selection of consumption modes.

consumers' groups are aggregated respectively. Table 6.2 and Table 6.3 illustrate the minimum adjusted and maximum adjusted power demand respectively, relative to the actual one. The results for each group of consumers and consumption mode are shown.

Table 6.2: The minimum adjustments of power demand relative to the actual one based on selections of occupancy modes, heating/cooling modes and their combination. The results concern the CONTROL group and the consumers with FIXED, TIME OF USE and REAL TIME PRICING contract.

\begin{tabular}{|l|c|c|c|}
\cline { 2 - 4 } \multicolumn{1}{c|}{} & Occupancy Modes & Heating/Cooling Modes & Combined \\
\hline CONTROL & $47.3 \%$ & $13.0 \%$ & $51.9 \%$ \\
\hline FIXED & $61.1 \%$ & $19.7 \%$ & $63.2 \%$ \\
\hline TIME OF USE & $50.2 \%$ & $26.6 \%$ & $62.7 \%$ \\
\hline REAL TIME PRICING & $33.6 \%$ & $30.9 \%$ & $48.4 \%$ \\
\hline
\end{tabular}

For the occupancy modes, the consumers with the REAL TIME PRICING contract have the lowest minimum and maximum adjustments of power demand of $33.6 \%$ and $33.8 \%$ respectively, relative to the actual power demand. The low adjustment potential of the REAL TIME PRICING group compared to the other groups of consumers is explained by the fact that this is the group that achieves the highest adjustments of power demand within the Olympic Peninsula Project [Hammerstrom, 2007]. The highest minimum and maximum adjustments are $61.1 \%$ and $51.7 \%$ respectively, relative to the actual 
Table 6.3: The maximum adjustments of power demand relative to the actual one based on selections of occupancy modes, heating/cooling modes and their combination. The results concern the CONTROL group and the consumers with FIXED, TIME OF USE and REAL TIME PRICING contract.

\begin{tabular}{|l|c|c|c|}
\cline { 2 - 4 } \multicolumn{1}{c|}{} & Occupancy Modes & Heating/Cooling Modes & Combined \\
\hline CONTROL & $43.0 \%$ & $7.6 \%$ & $46.2 \%$ \\
\hline FIXED & $51.7 \%$ & $16.7 \%$ & $57.1 \%$ \\
\hline TIME OF USE & $48.3 \%$ & $17.1 \%$ & $54.7 \%$ \\
\hline REAL TIME PRICING & $33.8 \%$ & $10.4 \%$ & $39.0 \%$ \\
\hline
\end{tabular}

power demand. These highest adjustments correspond to the consumers with a FIXED contract.

For the heating/cooling modes, the highest minimum adjustment of power demand is $30.9 \%$ relative to the actual power demand for the consumers with a REAL TIME PRICING contract. The highest maximum adjustment of power demand is $17.1 \%$ relative to the actual power demand for the consumers with a TIME OF USE contract. Moreover, the lowest minimum and maximum adjustment of power demand is $13.0 \%$ and $7.6 \%$ respectively, relative to the actual power demand. These lowest adjustments correspond to the consumers of the CONTROL group.

Note that although the consumers with REAL TIME PRICING contracts have the lowest minimum adjustment of power demand based on selections of occupancy modes, the opposite holds for selections of heating/cooling modes. Consumers with REAL TIME PRICING contracts have for each occupancy mode two temperature ranges, one for heating and one for cooling. A switch from a heating to a cooling mode is an actual utilization of an extended temperature range that is larger than switching between different occupancy modes. These switches are observed within the transition period autumn-winter during which the total power demand gradually increases and consumers may select both heating and cooling modes during the day.

Finally, the results concerning the combination of occupancy and heating/cooling modes are similar to the results of occupancy modes. The difference is that the adjustments are higher. The consumers with REAL TIME PRICING contract have the lowest minimum and maximum adjustments of power demand by $48.4 \%$ and $39.0 \%$ respectively, relative to the actual power demand. The highest minimum and maximum adjustments are $63.2 \%$ and $57.1 \%$ respectively, relative to the actual power demand. These highest adjustments correspond to the consumers with a FIXED contract.

\subsubsection{Interpretation of results}

The analytical results computed using data from the Olympic Peninsula Smart Grid Demonstration Project confirm the hypothesis set in this section:

Hypothesis. Adjustments of aggregate energy consumption can be achieved with possible demand options of local energy consumption, representing a wide range of comfort and economy levels, that can be pre-defined and dynamically selected by incentivized consumers.

In all of the demand options studied, i.e., the occupancy modes, the heating/cooling modes and their combination, a significant level of adjustments in the aggregate energy 
consumption is possible. The higher the number of consumption modes that consumers configure, the higher the potential for adjustments in the energy consumption is. The combination of occupancy and heating/cooling modes results in the highest adjustments of energy consumption compared to each ones individually.

Moreover, the analytical results show that a significant degree of adjustment in energy consumption is unexploited by the group of consumers with a FIXED and TIME OF USE contracts. The group of consumers with a REAL TIME PRICING contract is better incentivized within the Olympic Peninsula Project, however, further adjustments can be achieved even in this group according to the analytical results computed. Finally, various temporal and regional factors, such as weather conditions, influence adjustments of energy consumption. For example, higher adjustment is observed during transition periods in which consumers behave more unpredictable and vary their energy consumption significantly during their day, e.g., from autumn to winter.

ALMA is able to compute and aggregate these adjustments in the power demand in a fully decentralized fashion without the need of a centralized aggregator as the Olympic Peninsula Project imposes. This is because of the DIAS overlay service. Information about demand is made locally available to consumers that have the option to react to a power peak or an excessive micro-generation. This is possible by adapting their comfort and economy levels via dynamic selections between the possible states of DIAS. In contrast to ALMA, the actual selections of comfort and economy levels in the Olympic Peninsula Project are static and pre-defined. The actual adjustments achieved in the project are limited compared to the ones computed in this section.

\subsection{Comparison with Related Work}

The main advantages of EPOS and ALMA compared to other related approaches of demand-side energy management are the following: (i) decentralization and (ii) capturing different levels of consumer involvement and participation. EPOS and ALMA manage (i) stabilization and (ii) adjustment of energy consumption respectively. The scope of these mechanisms is broader compared to related methodologies that focus exclusively on load-shifting [Stadler et al., 2009, Shaw et al., 2009, Lu et al., 2005, Middelberg et al., 2009, Bakker et al., 2010], peak shaving [Strengers, 2008, Ashok, 2006, Faruqui and George, 2005, Hopper et al., 2006, McDonough and Kraus, 2007] or the utilization of renewable energy resources [Erol-Kantarci et al., 2011, Brandstätt et al., 2011, Paulus and Borggrefe, 2011].

Load-shifting is the stabilization approach that is closest to the concept of EPOS. In the work of Stadler et al. [2009], cooling devices, such as refrigerators, are assumed to respond to signals from the power grid. Energy consumption is decreased during peak times or the 'on' states of the controlled devices are shifted to periods with low energy demand. However, the whole process is centrally controlled without any coordination and interactions between the responding devices. For example, it is not clear what happens when devices shift their consumption to another time period resulting in a shift of the peak. This is the 'rebound effect' discussed by Palensky and Dietrich [2011]. Similarly, Lu et al. [2005] study and model the flexibility of thermostatically controlled appliances for load-shifting, however, coordination between devices is not addressed. Finally, Bakker et al. [2010] introduce a three-step optimization method performed with the support of a tree structure, similarly to EPOS. The root of the tree has the role of 
global planner and coordinates the optimization in a top-down fashion using prediction information in each of its planners bellow. Load-shifting is achieved on the basis of minimizing the energy cost of consumers, in contrast to EPOS that uses alternative equivalent plans.

Centralized coordination approaches can achieve optimal control and load-shifting under certain conditions. For example, Middelberg et al. [2009] propose such an approach based on a binary integer programming problem solved with existing methods. The model is applied for the management of a colliery. A similar integer programming model is proposed by Ashok [2006] for the management of steel plants. In contrast to EPOS and ALMA, these centralized methods are suitable and scalable within closed industrial environments rather than within a large-scale environment of residential consumers.

Adjustments of power demand based on price incentives are usually achieved within a centralized two-way market between consumers and utility companies. Examples of such markets are illustrated by Kok et al. [2005], Hammerstrom [2007], Faruqui and George [2005] and Hopper et al. [2006]. This centralized approach has a significant impact on scalability and privacy as discussed in Section 6.2. In contrast, ALMA introduces a decentralized aggregation and adaptation of power demand via interactions between consumers with a minimum intervention of their utility companies.

\subsection{Discussion and Future Work}

This chapter shows that demand-side energy management is a complex socio-technical problem with challenges crossing a wide range of stakeholders in this domain. Consumers are larger in number, more distributed and dynamic compared to producers. Furthermore, consumers evolve to prosumers having the option to produce energy as well and actively participate in energy markets. Using overlay services for demandside energy management is a promising approach. Knowledge transfer from the domain of distributed computing to the evolving domain of Smart Power Grid provides new technical insights and the means for large-scale decentralized demand-side energy management. Both EPOS and ALMA are based on overlay services that interconnect consumers to collectively stabilize and adapt their energy consumption with minimum interventions from the supply-side.

EPOS shows that within a large-scale tree overlay network of thermostatically controlled devices, decentralized coordination minimizes or reverses deviation of energy consumption without the direct involvement of consumers. EPOS achieves sub-optimum stabilization, however, the degree of stabilization achieved depends on performance trade-offs related to the energy plans and the topology organization. Future work focuses on the impact of the tree ordering on the achieved stabilization by using different application criteria in AETOS. Other fitness functions need to be designed as well capable to meet more complex system objectives, e.g., functions that predict generation from renewable energy resources. Duration of the energy plans needs to be evaluated in practice. Typical choices observed in literature are 5 minutes [Hammerstrom, 2007], 15 minutes [Hammerstrom et al., 2010], 60 minutes [Hopper et al., 2006] or even a day-ahead planning [Faruqui and George, 2005].

ALMA goes one step further and complements EPOS by actively involving consumers to adjust the aggregate energy consumption. This potential contributes to the robust- 
ness of the Smart Power Grid and prevents system blackouts [Pournaras et al., 2012]. Adjustments are achieved by making locally available the aggregate consumption to consumers in order to adapt their selected demand according to economic or other incentives. Selections represent the trade-off between comfort and economy. An analysis of the power demand during the Olympic Peninsula Project [Hammerstrom et al., 2010] shows that adjustment of power demand is technically possible using the decentralized approach of ALMA. A further analysis of the semantic that occupancy modes have for each consumer may provide a better understanding of the consumption behavior. Moreover, subject of future work is the exact economic and other incentives that should be designed to meet the maximum possible technical adjustments in power demand. An interesting aspect that needs investigation is the degree to which decentralization itself is an incentive or not for consumers to participate in demand/response programs.

Finally, note that the introduction of such highly dynamic and decentralized mechanisms in a critical infrastructure such as the Smart Power Grid raises crucial cybersecurity issues [Khurana et al., 2010]. Although solutions on security issues are out of the scope of this thesis, some challenges that need to be addressed include the integration of existing computer and radio security countermeasures in the Smart Power Grid and the engineering of new cyber-security systems for Internet-scale control systems. Security technologies may require new policies and expertise in the domain of the Smart Power Grid. For example Metke and Ekl [2010] mention that system operators need to acquire knowledge about security systems such as the Public Key Infrastructure (PKI).

\subsection{Conclusions}

Compared to production-side, demand-side energy management becomes a more costeffective approach to prevent cascading failures, mitigate blackouts, and make the Smart Power Grid more robust, adaptive, environmental-friendly and sustainable. This chapter concludes that the use of overlay services in emerging application domains such as the Smart Power Grid provide new insights about how to manage the energy from demand-side. The benefits of overlay services used in EPOS, the Energy Plan Overlay Self-stabilization and ALMA, the Adaptive Load Management by Aggregation are twofold: (i) decentralization that provides a higher scalability, autonomy, fault tolerance together with (ii) two automation levels of consumer involvement in demand-side energy management.

EPOS is based on the AETOS overlay service and stabilizes the energy consumption by minimizing or reversing its deviation over time. The consumption of thermostatically controlled appliances is coordinated in a decentralized fashion transparently from consumers using software agents. However, in cases that an actual reduction of the energy consumption is required and, consequently, a change in the consumption behavior of consumers, EPOS is not adequate. ALMA bridges this gap based on the DIAS overlay service. The analytical study illustrated in this chapter, grounded to the current reality and practice of Smart Power Grids [Hammerstrom, 2007], shows that adjustments in the aggregated energy consumption are possible. Compared to related methodologies, EPOS and ALMA extend the technical scope of demand-side energy management in different consumer groups and motivate alternative business and market structures for adoption in the future Smart Power Grids. 



\title{
Chapter 7

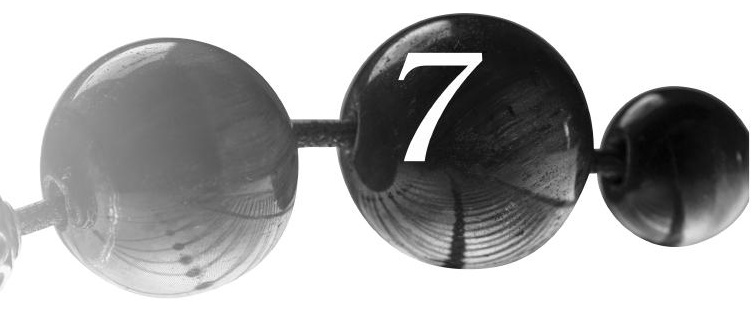

\section{Conclusions and Future Work}

\author{
"No power and no treasure can outweigh the extension of our \\ knowledge."
}

Democritus $^{1}$

This thesis studies generic application capabilities for large-scale decentralized systems organized in overlay networks: the overlay services. The main contribution of this thesis is a conceptual architecture to build overlay services: ASMA, the Adaptive Self-organization in a Multi-level Architecture, introduced in Chapter 3. The ASMA architecture supports the design of generic large-scale decentralized systems of overlay networks. This is achieved by introducing the critical operations of discovery, structuring and coordination in three adaptive and reconfigurable self-organization levels that provide abstraction and modularity.

This contribution advances the state of the art in the research area of large-scale decentralized systems by introducing, in these systems, two generic capabilities as overlay services: (i) self-organization of overlay networks in different tree topologies that match different application criteria and (ii) computation of different aggregation functions over a set of dynamically changing values distributed in an overlay network. These generic capabilities form AETOS, the Adaptive Epidemic Tree Overlay Service, introduced in Chapter 4 and DIAS, the Dynamic Intelligent Aggregation Service, introduced in Chapter 5.

This thesis studies the applicability of these two services in the domain of the Smart Power Grid. More specifically, AETOS and DIAS support two decentralized mechanisms for demand-side energy management introduced in Chapter 6: EPOS, the Energy Plan Overlay Self-stabilization and ALMA, the Adaptive Load Management by Aggregation. The energy management goal of EPOS is the self-stabilization of energy consumption without the direct involvement of consumers. In contrast, the goal of ALMA is the self-adjustment of energy consumption by incentivized consumers who choose between multiple demand options.

This thesis shows that the high-level algorithmic interface of ASMA is able to represent complex functionality of overlay services using a few lines of algorithmic expressions. Both AETOS and DIAS are instantiations of the same ASMA interface. Although

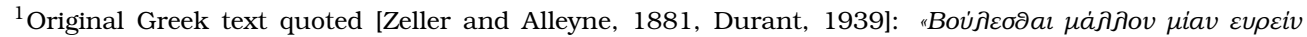

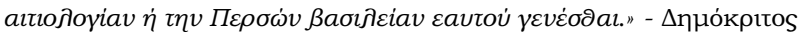


these two overlay services provide two significantly different application capabilities, they are based on the same architectural design of ASMA. AETOS and DIAS are a proof of concept for the ASMA architecture. In addition, they are also building examples towards other overlay services and generic distributed systems operating in large decentralized environments.

The overlay services of AETOS and DIAS use adaptation strategies as the means to perform a wide range of high-level automated reconfigurations by hiding technical details of system parameterization and optimization. In other words, adaptation strategies exploit the degree of freedom in the design of self-organization. For example, the eight adaptation strategies of AETOS result in tree topologies of different graph-properties without the need to redefine a fitness function or the interactions between agents. Similarly, the two adaptation strategies of DIAS perform aggregation that minimizes inaccuracies and performance downgrade originated by aggregation values that change or are not computed. The aggregation functions and interactions do not require any adjustment in this case. Note that adaptation strategies additionally introduce performance trade-offs that can be made during the runtime of an overlay service and, therefore, provide an intuition and a better understanding of complex system behavior. Finally, the experimental evaluation of AETOS and DIAS shows that dynamic adoption of an adaptation strategy is possible and may result in performance enhancements. For instance, the speed of sorting and connecting nodes in a tree topology of AETOS is maximized if more than one adaptation strategy are adopted during runtime.

The abstraction and modularity of ASMA unavoidably influences the performance of its instantiations. As a general conclusion, the related work of AETOS and DIAS provides solutions that require a lower cost, i.e., communication, but end with limited functionality or operational flexibility. In contrast, AETOS and DIAS require an initial higher cost mainly imposed by its multi-level design approach to expand the functionality and applicability scope. Yet, the experimental evaluation of the ASMA overlay services shows that initial high operational cost can be controlled and minimized by the adaptation strategies and the reconfigurations performed in each level instantiation. An example of the high reconfigurability in the performance of AETOS is the switch of adaptation strategy to rapidly merge disconnected branches into one (tree) component (Section 4.7.4). Similarly, the allocation of storage space in the bloom filters of DIAS is performed based on the expectation of certain false positive probability (Section 5.8.3).

The applicability of AETOS and DIAS in the Smart Power Grid shows that overlay services technically enable household consumers to play a more active and engaging, yet flexible, role. This role fulfills a plethora of sustainability requirements related to a more efficient and environmental-friendly energy usage, e.g., stabilization/adjustment of energy consumption and integration of renewable energy resources or electrical vehicles. The experimental and analytical evaluation performed to EPOS and ALMA shows that demand-response programs can become more cost-effective with a higher impact on the robustness of the Smart Power Grid if they allow a more flexible involvement of consumers compared to existing highly committing programs and static policies.

\subsection{Meeting Research Objectives}

Recall from Section 1.2 the main research question of this thesis: 
Question. Can large-scale decentralized networked systems be designed to provide modular and reconfigurable overlay services?

The conceptual architecture of ASMA introduced in Chapter 3 shows that generic capabilities for large-scale decentralized systems can be designed by performing a multilevel (i) discovery, (ii) structuring and (iii) coordination of system entities and their information based on self-organization criteria. Generic overlay services require an abstraction and modularity that the three self-organization levels, their interactions and interoperation provide. The ASMA architecture fills the conceptual gap of abstraction, modularity and reconfigurability in the design of overlay services.

The generic design concept of ASMA is studied and validated in the two overlay service instantiations of AETOS and DIAS. The design and applicability scope of each of these overlay services is broader compared to the current practice and state of the art. In AETOS, different graph properties are acquired in the self-organization of tree topologies and in DIAS, different aggregation functions can be computed in a decentralized fashion without relying on a specific routing mechanism.

Furthermore, by studying the performance of these services in detail, this thesis shows that the impact of the introduced abstraction and modularity may unavoidably degrade performance, i.e., by increasing the communication cost. Yet, the high degree of reconfigurability in each level instantiation of ASMA allows performance enhancements based on trade-offs. For example, the trade-off between convergence speed and communication cost in the self-organization of AETOS, or the trade-off between accuracy of aggregates and the storage space in DIAS can be both managed within the instantiated architecture of ASMA.

The applicability of these overlay services, illustrated in Chapter 6, shows that ASMA and its realizations provide decentralized computing solutions in two challenging problems of demand-side energy management: (i) self-stabilization and (ii) self-adjustment of energy consumption. The actual design approach of the ASMA conceptual architecture is the intuition behind these decentralized solutions. This is because the applicability of overlay services, such AETOS and DIAS, in the energy domain is an actual proof of concept for the benefits and challenges that decentralized computing introduces in the Smart Power Grid.

\subsection{Open Issues and Future work}

This section outlines several research aspects of the overlay services and demand-side energy management mechanisms that need to be further studied and addressed. Note that each of these overlay services and mechanisms could be individually studied in more depth as a separate thesis. However, this thesis builds knowledge that covers a broader research spectrum of large-scale decentralized systems by focusing on their generic design, yet, studying their applicability in the critical application domain of the Smart Power Grid.

The conceptual architecture of ASMA can be further validated with other overlay services. Moreover, it is crucial to show if the three levels of ASMA are adequate in other overlay service instantiations, or if additional levels need to be introduced to capture more complex functionality and requirements beyond discovery, structuring and coordination. Moving the conceptual architecture of ASMA closer to a more complete 
framework implementation requires the evaluation and selection of relevant software technologies [Tanenbaum and Van Steen, 2007].

The complex system behavior in the overlay services of AETOS and DIAS is mainly studied experimentally. This approach provides plenty of intuition towards a solid and in-depth mathematical formulation. The topological effect of each adaptation strategy in AETOS, the convergence speed of AETOS, the relation of the false positive probability with the accuracy of aggregation in DIAS are some examples in which formal and analytical methods may provide a more complete understanding. Furthermore, an actual deployment and emulation of AETOS and DIAS in a testbed of a global scale such as PlanetLab can provide broader insights about the behavior and lifecycle of these complex systems during their operation in less controlled environments.

Further work on the fault-tolerance of overlay services is required. Despite the fact that the gossiping realization of the discovery level provides a highly robust overlay interconnection, failures unavoidably affect the quality of an overlay service and therefore the application performance. For example, failures in the nodes of AETOS may result in erroneous stabilization of energy consumption in EPOS. Similarly, such failures in DIAS originate inaccurate aggregate information that may mislead the adjustment of power demand by consumers in ALMA. A critical design choice concerns the positioning of fault tolerance as (i) an application-level solution, (ii) an overlay service or (iii) an integrated part of the ASMA architecture.

Decentralized systems and especially overlay networks face numerous security challenges as illustrated in Section 2.5 and 6.6. Security is one of the main concerns for the adoption of such systems in reality and especially in critical application domains such as the Smart Power Grid. Therefore, making the contributions of this research work more applicable requires its positioning in a more well-defined security context.

Moving closer to current reality and practice of Smart Power Grid requires the deployment and evaluation of mechanisms such as EPOS and ALMA within demonstration projects, i.e., Olympic Peninsula [Hammerstrom, 2007] and Pacific Northwest ${ }^{2}$. This practice is not only a roadmap for technological adoption but also a research platform that provides new insights and knowledge about problems that are usually assumed not relevant in earlier research phases.

In conclusion, this thesis indicates that introducing decentralized computing systems in an information era expanding to new critical application domains, such as the Smart Power Grid, is a promising endeavor towards more sustainable development and a resource-based economy in future societies.

${ }^{2}$ Available at: http: //www.pnwsmartgrid.org (Last accessed: August 2012) 


\section{Bibliography}

K. Aberer, L. Alima, A. Ghodsi, S. Girdzijauskas, S. Haridi, and M. Hauswirth. The Essence of P2P: A Reference Architecture for Overlay Networks. In Proceedings of the 5th International Conference on Peer-to-Peer Computing, P2P 2005, pages 11-20, Los Alamitos, CA, USA, Sept. 2005. IEEE.

J. Agel. Pearls of Wisdom: A Harvest of Quotations from All Ages. HarperCollins, 1987.

L. AlAbdulkarim and Z. Lukszo. Integrating information security requirements in critical infrastructures: smart metering case. International Journal of Critical Infrastructures, 6(2):187-209, Apr. 2010.

M. Alahmad, W. Nader, Y. Cho, J. Shi, and J. Neal. Integrating physical and virtual environments to conserve energy in buildings. Energy and Buildings, 43(12):37103717, Dec. 2011.

M. Albadi and E. El-Saadany. A summary of demand response in electricity markets. Electric Power Systems Research, 78(11):1989-1996, Nov. 2008.

R. Ambrosio, A. Morrow, and N. Noecker. e-Business Control Systems. In Proceedings of the 2nd International Conference on Computing, Communications, and Control Technologies, CCCT 2011, pages 91-96, Austin, TX, USA, Mar. 2011. IEEE Computer Society.

T. Anderson, L. Peterson, S. Shenker, and J. Turner. Overcoming the Internet Impasse through Virtualization. Computer, 38(4):34-41, Apr. 2005.

M. Artigas, P. García, and A. Skarmeta. DECA: A Hierarchical Framework for DECentralized Aggregation in DHTs. In Proceedings of the 17th IFIP/IEEE International Workshop on Distributed Systems: Operations and Management, DSOM 2006, volume 4269 of Lecture Notes in Computer Science, pages 246-257, Heidelberg, Oct. 2006. Springer-Verlag Berlin.

S. Ashok. Peak-load management in steel plants. Applied Energy, 83(5):413-424, May 2006.

J. Aspnes and G. Shah. Skip Graphs. ACM Transactions on Algorithms, 3(4):37:1-37:25, Nov. 2007.

S. Baek, G. DeVeciana, and X. Su. Minimizing Energy Consumption in Large-Scale Sensor Networks Through Distributed Data Compression and Hierarchical Aggregation. IEEE Journal on Selected Areas in Communications, 22(6):1130-1140, Aug. 2004. 
P. Bahl, P. Barham, R. Black, R. Chandra, M. Goldszmidt, R. Isaacs, S. Kandula, L. Li, J. Maccormick, D. Maltz, R. Mortier, M. Wawrzoniak, and M. Zhang. Discovering Dependencies for Network Management. In Proceedings of the 5th ACM Workshop on Hot Topics in Networking, HotNets 2006, pages 97-102, Irvine, California, Nov. 2006. ACM.

V. Bakker, M. Bosman, A. Molderink, J. Hurink, and G. Smit. Demand side load management using a three step optimization methodology. In Proceedings of the 1st International Conference on Smart Grid Communications, SmartGridComm 2010, pages 431-436, Oct. 2010.

D. Baldwin and G. Scragg. Algorithms and Data Structures: The Science of Computing. Charles River Media, Inc., Rockland, MA, USA, June 2004.

S. Balsamo, A. Di Marco, P. Inverardi, and M. Simeoni. Model-Based Performance Prediction in Software Development: A Survey. IEEE Transactions on Software Engineering, 30(5):295-310, May 2004.

S. Banerjee, C. Kommareddy, K. Kar, B. Bhattacharjee, and S. Khuller. Construction of an Efficient Overlay Multicast Infrastructure for Real-time Applications. In Proceedings of the 22nd Annual Joint Conference of the IEEE Computer and Communications Societies, INFOCOM 2003, pages 1521-1531, Los Alamitos, CA, USA, 2003. IEEE.

A. G. Beltran, P. Sage, and P. Milligan. Skip Tree Graph: a Distributed and Balanced Search Tree for Peer-to-Peer Networks. In Proceedings of the International Conference on Communications, ICC 2007, pages 1881-1886, Los Alamitos, CA, USA, June 2007. IEEE.

R. Bhagwan, S. Savage, and G. Voelker. Understanding Availability. In Proceedings of Peer-to-Peer Systems II, Second International Workshop, IPTPS 2003, volume 2735 of Lecture Notes in Computer Science, pages 256-267, Heidelberg, Oct. 2003. SpringerVerlag Berlin.

K. Birman. Technology Challenges for Virtual Overlay Networks. IEEE Transactions on Systems, Man, and Cybernetics - Part A: Systems and Humans, 31(4):319-327, July 2001.

S. Birrer and F. Bustamante. A Comparison of Resilient Overlay Multicast Approaches. IEEE Journal on Selected Areas in Communications, 25(9):1695-1705, Dec. 2007.

B. H. Bloom. Space/time trade-offs in hash coding with allowable errors. Communications of the ACM, 13(7):422-426, July 1970.

A. Boulis, S. Ganeriwal, and M. B. Srivastava. Aggregation in sensor networks: an energy-accuracy trade-off. Ad Hoc Networks, 1(2-3):317-331, Sept. 2003.

C. Brandstätt, G. Brunekreeft, and K. Jahnke. How to deal with negative power price spikes? Flexible voluntary curtailment agreements for large-scale integration of wind. Energy Policy, 39(6):3732-3740, June 2011.

R. Braynard, D. Kostic, A. Rodriguez, J. Chase, and A. Vahdat. Opus: an Overlay Peer Utility Service. In Proceedings of Open Architectures and Network Programming, OPENARCH 2002, pages 167-178, Los Alamitos, CA, USA, Jan. 2002. IEEE. 
O. Brendel. Symbolism of the Sphere: A Contribution to the History of Earlier Greek Philosoph. E. J. Brill, Leiden, The Netherlands, 1977.

A. Brodera and M. Mitzenmacher. Network Applications of Bloom Filters: A Survey. Internet Mathematics, 4(1):485-509, Jan. 2004.

J. Burnet. The Ethics of Aristotle: The Nicomachean Ethics. Methuen, 1900.

T. Calvo, A. Kolesárová, M. Komorníková, and R. Mesiar. Aggregation Operators: Properties, Classes and Construction Methods. In Aggregation Operators: New Trends and Applications, volume 97 of Studies in Fuzziness and Soft Computing, chapter 1, pages 3-104. Physica-Verlag GmbH, Heidelberg, Germany, Jan. 2002.

F. Cantin, B. Gueye, M. Kaafar, and G. Leduc. A Self-Organized Clustering Scheme for Overlay Networks. In Proceedings of the 3rd International Workshop on Self-Organizing Systems, volume 5343 of Lecture Notes in Computer Science, pages 59-70, Heidelberg, Nov. 2008. Springer Berlin.

P. Cappers, C. Goldman, and D. Kathan. Demand response in U.S. electricity markets: Empirical evidence. Energy, 35(4):1526-1535, Apr. 2010.

M. Castro, M. Costa, and A. Rowstron. Debunking some myths about structured and unstructured overlays. In Proceedings of the 2nd International Symposium on Networked Systems Design \& Implementation, NSDI 2005, pages 85-98, Berkeley, CA, USA, May 2005. USENIX Association.

C. P. Cavafy. C.P. Cavafy: Collected Poems. Princeton University Press, revised edition, 1992. translated by Edmund Keeley and Philip Sherrard.

A. J. Chakravarti, G. Baumgartner, and M. Lauria. Self-Organizing Scheduling on the Organic Grid. International Journal of High Performance Computing Applications, 20 (1):115-130, Feb. 2006.

H. Chang, S. Jamin, and W. Willinger. To Peer or Not to Peer: Modeling the Evolution of the Internet's AS-Level Topology. In Proceedings of the 25th IEEE International Conference on Computer Communications, INFOCOM 2006, pages 1-12, Los Alamitos, CA, USA, Apr. 2006. IEEE.

L. Chitnis, A. Dobra, and S. Ranka. Aggregation Methods for Large-Scale Sensor Networks. ACM Transactions on Sensor Networks, 4(2):1-36, Mar. 2008.

H. Choe, S. Cho, and C.-k. Kim. A Dynamic Mechanism for Distributed Optimization of Overlay Multicast Tree. In Proceedings of the International Conference on Information Networking, ICOIN 2004, Heidelberg, 2004. Springer-Verlag Berlin.

N. Chowdhury and R. Boutaba. Network Virtualization: State of the Art and Research Challenges. IEEE Communications Magazine, 47(7):20-26, July 2009.

D. Clark, B. Lehr, S. Bauer, P. Faratin, R. Sami, and J. Wroclawski. Overlay Networks and the Future of the Internet. Communications \& Strategies, (63):109-129, July 2006. 
K. Clement-Nyns, E. Haesen, and J. Driesen. The Impact of Charging Plug-In Hybrid Electric Vehicles on a Residential Distribution Grid. IEEE Transactions on Power Systems, 25(1):371-380, Feb. 2010.

L. Colitti, S. H. Gunderson, E. Kline, and T. Refice. Evaluating IPv6 Adoption in the Internet. In Proceedings of the 11th International Conference on Passive and Active Measurement, PAM 2010, volume 6032 of Lecture Notes in Computer Science, pages 141-150, Heidelberg, Apr. 2010. Springer-Verlag Berlin.

A. Corez and F. Robledo. Multi-overlay Network Planning by applying a Variable Neighborhood Search approach. In Proceedings of the 3rd Latin-American Conference on Communications, LATINCOM 2011, pages 1-6, Los Alamitos, CA, USA, Oct. 2011. IEEE.

P. Costa and D. Frey. Publish-Subscribe Tree Maintenance over a DHT. In Proceedings of the 4th International Workshop on Distributed Event-Based System, DEBS 2005, pages 414-420, Washington, DC, USA, 2005. IEEE Computer Society.

J. W. Creswell. Research Design: Qualitative, Quantitative, and Mixed Method Approaches. Sage Publications, 3rd edition, 2008.

F. Crossan. Research philosophy: towards an understanding. Nurse researcher, 11(1): 46-55, Jan. 2003.

J. Crowcroft, S. Hand, R. Mortier, T. Roscoe, and A. Warfield. Plutarch: An Argument for Network Pluralism. In Proceedings of the ACM SIGCOMM Workshop on Future Directions in Network Architecture, FDNA 2003, volume 33, pages 258-266, New York, NY, USA, Aug. 2003. ACM Press.

G. Deke, L. Yunhao, L. Xiangyang, and Y. Panlong. False Negative Problem of Counting Bloom Filter. IEEE Transactions on Knowledge and Data Engineering, 22(5):651-664, May 2010.

P. C. Dillinger and P. Manolios. Bloom Filters in Probabilistic Verification. In Proceedings of the 5th International Conference on Formal Methods in Computer-Aided Design, FMCAD 2004, volume 3312 of Lecture Notes in Computer Science, pages 367-381, Heidelberg, Jan. 2004. Springer-Verlag, Berlin.

A. Dimeas and N. Hatziargyriou. Agent based control of Virtual Power Plants. In Proceedings of the International Conference on Intelligent Systems, ISAP 2007, pages 536-541, Los Alamitos, CA, USA, Nov. 2007. IEEE.

C. Diot, B. Levine, B. Lyles, H. Kassem, and D. Balensiefen. Deployment Issues for the IP Multicast Service and Architecture. IEEE Network, 14(1):78-88, Jan. 2000.

W. Durant. The Story of Civilization: The Life of Greece. Simon \& Schuster, New York, 1939.

D. England, B. Veeravalli, and J. B. Weissman. A Robust Spanning Tree Topology for Data Collection and Dissemination in Distributed Environments. IEEE Transactions on Parallel and Distributed Systems, 18(5):608-620, May 2007. 
M. Erol-Kantarci, B. Kantarci, and H. Mouftah. Reliable Overlay Topology Design for the Smart Microgrid Network. IEEE Network, 25(5):38-43, Sept. 2011.

G. Exarchakos, N. Antonopoulos, and J. Salter. G-ROME: semantic-driven capacity sharing among P2P networks. Internet Research, 17(1):7-20, June 2007.

A. Fairbanks. The first philosophers of Greece. English and foreign philosophical library. K. Paul, Trench, Trübner \& co., ltd., 1898.

J. Fan and M. H. Ammar. Dynamic Topology Configuration in Service Overlay Networks: A Study of Reconfiguration Policies. In Proceedings of the 25th International Conference on Computer Communications, INFOCOM 2006, pages 1-12, Los Alamitos, CA, USA, Apr. 2006. IEEE.

Z. Fan, G. Kalogridis, C. Efthymiou, M. Sooriyabandara, M. Serizawa, and J. McGeehan. The New Frontier of Communications Research: Smart Grid and Smart Metering. In Proceedings of the 1st International Conference on Energy-Efficient Computing and Networking, e-Energy 2010, pages 115-118, New York, NY, USA, Apr. 2010. ACM Press.

A. Faruqui and S. George. Quantifying Customer Response to Dynamic Pricing. The Electricity Journal, 18(4):53-63, May 2005.

E. Fasolo, M. Rossi, J. Widmer, and M. Zorzi. In-network Aggregation Techniques for Wireless Sensor Networks: A Survey. IEEE Wireless Communications, 14(2):70-87, Apr. 2007.

A. Fei, J. Cui, M. Gerla, and M. Faloutsos. Aggregated Multicast with Inter-Group Tree Sharing. In Proceedings of the 3rd International Workshop on Networked Group Communication, NGC 2001, volume 2233 of Lecture Notes in Computer Science, pages 172-188, Heidelberg, Oct. 2001. Springer-Verlag Berlin.

Z. Fei and M. Yang. A Proactive Tree Recovery Mechanism for Resilient Overlay Multicast. IEEE/ACM Transactions on Networking, 15(1):173-186, Feb. 2007.

P. Flajolet and G. Nigel Martin. Probabilistic Counting Algorithms for Data Base Applications. Journal of Computer and System Sciences, 31(2):182-209, Oct. 1985.

D. Frey and A. L. Murphy. Failure-Tolerant Overlay Trees for Large-Scale Dynamic Networks. In Proceedings of the International Conference on Peer-to-Peer Computing, pages 351-361, Los Alamitos, CA, USA, Sept. 2008. IEEE.

W. Galuba, K. Aberer, Z. Despotovic, and W. Kellerer. ProtoPeer: A P2P Toolkit Bridging the Gap Between Simulation and Live Deployement. In Proceedings of the Second International Conference on Simulation Tools and Techniques, ICST 2009, pages 1-9, Gent, Belgium, Mar. 2009. ACM.

P. Garbacki, D. H. J. Epema, and M. van Steen. The Design and Evaluation of a SelfOrganizing Superpeer Network. IEEE Transactions on Computers, 59(3):317-331, Mar. 2010. 
F. Garcin, B. Faltings, R. Jurca, and N. Joswig. Rating Aggregation in Collaborative Filtering Systems. In Proceedings of the 3rd ACM Conference on Recommender Systems, RecSys 2009, pages 349-352, New York, NY, USA, Oct. 2009. ACM Press.

I. Giannoccaro and P. Pontrandolfo. Negotiation of the revenue sharing contract: An agent-based systems approach. International Journal of Production Economics, 122 (2):558-566, Dec. 2009.

C. Gkantsidis, M. Mihail, and S. Saberi. Random walks in peer-to-peer networks: Algorithms and evaluation. Performance Evaluation, 63(3):241-263, Mar. 2006.

A. Goel and P. Gupta. Small subset queries and bloom filters using ternary associative memories, with applications. ACM SIGMETRICS Performance Evaluation Review, 38 (1): 143, June 2010.

A. González-Beltrán, P. Milligan, and P. Sage. Range queries over skip tree graphs. Computer Communications, 31(2):358-374, Feb. 2008.

P. Grace, G. Coulson, G. S. Blair, L. Mathy, W. Kit Yeung, W. Cai, D. A. Duce, and C. S. Cooper. GRIDKIT: Pluggable Overlay Networks for Grid Computing. In Proceedings of Cooperative Information Systems, CoopIS 2004, volume 3291 of Lecture Notes in Computer Science, pages 1463-1481, Heidelberg, Oct. 2004. Springer-Verlag Berlin.

P. Grace, D. Hughes, B. Porter, G. S. Blair, G. Coulson, and F. Taiani. Experiences with Open Overlays: A Middleware Approach to Network Heterogeneity. In Proceedings of the 3rd European Conference on Computer Systems, SIGOPS/EuroSys 2008, pages 123-136, New York, USA, Apr. 2008. ACM.

S. Grijalva and M. U. Tariq. Prosumer-based Smart Grid Architecture Enables a Flat, Sustainable Electricity Industry. In Proceedings of the Innovative Smart Grid Technologies, ISGT 2011, pages 1-6, Los Alamitos, CA, USA, Jan. 2011. IEEE.

J. Gross and J. Yellen. Graph Theory and Its Applications. CRC Press, Boca Raton, FL, USA, 2nd edition, Sept. 2005.

Y. Guo, J. Li, and G. James. Evolutionary Optimisation of Distributed Energy Resources. In Proceedings of the 18th Australian Joint Conference on Artificial Intelligence, volume 3809 of Lecture Notes in Computer Science, pages 1086-1091, Heidelberg, Nov. 2005. Springer Berlin.

D. Haage, R. Holz, H. Niedermayer, and P. Laskov. CLIO - A Cross-Layer Information Service for Overlay Network Optimization. In Kommunikation in Verteilten Systemen (KiVS), Informatik aktuell, pages 279-284, Heidelberg, Mar. 2009. Springer-Verlag Berlin.

S. W. Hadley and A. A. Tsvetkova. Potential Impacts of Plug-in Hybrid Electric Vehicles on Regional Power Generation. The Electricity Journal, 22(10):56-68, Dec. 2009.

D. J. Hammerstrom. Part I. Olympic Peninsula Project. Technical report, Pacific Northwest National Laboratory, Richland, WA, USA, Oct. 2007. 
D. J. Hammerstrom, T. V. Oliver, R. B. Melton, and R. Ambrosio. Standardization of a Hierarchical Transactive Control System. In Proceedings of the Grid Interop 2009, Richland, WA, USA, Dec. 2010. GridWise Architecture Council.

M. Haridasan and R. van Renesse. Gossip-based Distribution Estimation in Peer-to-Peer Networks. In Proceedings of the 7th International Workshop on Peer-to-peer Systems, IPTPS 2008, page 13, Berkeley, CA, USA, Feb. 2008. USENIX Association.

N. J. A. Harvey, M. B. Jones, S. Saroiu, M. Theimer, and A. Wolman. SkipNet: A Scalable Overlay Network with Practical Locality Properties. In Proceedings of the 4th Conference on USENIX Symposium on Internet Technologies and Systems, USITS 2003, page 9, Berkeley, CA, USA, Mar. 2003. USENIX Association.

A. R. Hevner, S. T. March, J. Park, and S. Ram. Design science in information systems research. MIS Quarterly, 28(1):75-105, Mar. 2004.

M. Holzer, F. Schulz, and D. Wagner. Engineering Multi-Level Overlay Graphs for Shortest-Path Queries. Journal of Experimental Algorithmics, 13(5):5:2.5-5:2.26, Aug. 2009.

W. Honghao, Z. Yingwu, and H. Yiming. To Unify Structured and Unstructured P2P Systems. In Proceedings of the 19th International Parallel and Distributed Processing Symposium, IPDPS 2005, page 104a, Los Alamitos, CA, USA, Apr. 2005. IEEE.

N. Hopper, C. Goldman, R. Bharvirkar, and B. Neenan. Customer response to day-ahead market hourly pricing: Choices and performance. Utilities Policy, 14(2):126-134, June 2006.

J. W. Hui and D. E. Culler. IP is Dead, Long Live IP for Wireless Sensor Networks. In Proceedings of the 6th ACM conference on Embedded network sensor systems, SenSys 2008, pages 15-28, New York, NY, USA, Nov. 2008. ACM Press.

A. Ipakchi and F. Albuyeh. Grid of the Future. IEEE Power and Energy Magazine, 7(2): 52-62, Mar. 2009.

H. V. Jagadish, B. C. Ooi, and Q. H. Vu. BATON: a balanced tree structure for peerto-peer networks. In Proceedings of the 31st International Conference on Very Large Databases, VLDB 2005, pages 661-672, Trondheim, Norway, Aug. 2005. ACM.

H. V. Jagadish, B. C. Ooi, K.-L. Tan, B. H. Vu, and R. Zhang. Speeding up Search in Peer-to-Peer Networks with A Multi-way Tree Structure. In Proceedings of the International Conference on Management of Data, SIGMOD 2006, pages 1-12, New York, USA, June 2006a. ACM Press.

H. V. Jagadish, B. C. Ooi, Q. H. Vu, R. Zhang, and Z. Aoying. VBI-Tree: A Peer-to-Peer Framework for Supporting Multi-Dimensional Indexing Schemes. In Proceedings of the 22nd International Conference on Data Engineering, ICDE 2006, pages 34-34, Washington, DC, USA, 2006b. IEEE Computer Society.

G. James, D. Cohen, R. Dodier, G. Platt, and D. Palmer. A Deployed Multi-Agent Framework for Distributed Energy Applications. In Proceedings of the 5th International Conference on Autonomous Agents and Multiagent Systems, AAMAS 2006, pages 676678, New York, NY, USA, May 2006. ACM Press. 
M. Jelasity, A. Montresor, and O. Babaoglu. Gossip-Based Aggregation in Large Dynamic Networks. ACM Transactions on Computer Systems, 23(3):219-252, Aug. 2005.

M. Jelasity, S. Voulgaris, R. Guerraoui, A.-M. Kermarrec, and M. van Steen. Gossipbased Peer Sampling. ACM Transactions on Computer Systems, 25(3), Aug. 2007.

M. Jelasity, A. Montresor, and O. Babaoglu. T-Man: Gossip-based fast overlay topology construction. Computer Networks, 53(13):2321-2339, Aug. 2009.

Z. Jerzak and C. Fetzer. Bloom Filter Based Routing for Content-Based Publish/Subscribe. In Proceedings of the 2nd International Conference on Distributed Event-based Systems, DEBS 2008, pages 71-81, New York, NY, USA, July 2008. ACM Press.

S. Jiang, L. Guo, and X. Zhang. LightFlood: an Efficient Flooding Scheme for File Search in Unstructured Peer-to-Peer Systems. In Proceedings of the 2003 International Conference on Parallel Processing, ICPP 2003, pages 627-635, Los Alamitos, CA, USA, Oct. 2003. IEEE.

L. Joita, O. F. Rana, P. Chacín, I. Chao, F. Freitag, L. Navarro, and O. Ardaiz. Application deployment using catallactic Grid middleware. In Proceedings of the 3rd International Workshop on Middleware for Grid Computing, MGC 2005, pages 1-6, New York, USA, Nov. 2005. ACM Press.

D. Joseph, J. Kannan, A. Kubota, K. Lakshminarayanan, I. Stoica, and K. Wehrle. OCALA: An Architecture for Supporting Legacy Applications over Overlays. In Proceedings of the 3rd International Conference on Networked Systems Design \& Implementation, NSDI 2006, page 20, Berkeley, CA, USA, May 2006. USENIX Association.

P. Joskow and J. Tirole. Reliability and Competitive Electricity Markets. The RAND Journal of Economics, 38(1):60-84, May 2007.

M. Kaashoek, I. Stoica, F. Dabek, B. Zhao, P. Druschel, and J. Kubiatowicz. Towards a Common API for Structured Peer-to-Peer Overlays. In Proceedings of Peer-to-Peer Systems II, Second International Workshop, IPTPS 2003, volume 2735 of Lecture Notes in Computer Science, pages 33-44, Heidelberg, 2003. Springer-Verlag Berlin.

A. Kailas, V. Cecchi, and A. Mukherjee. A Survey of Communications and Networking Technologies for Energy Management in Buildings and Home Automation. Journal of Computer Networks and Communications, 2012:1-12, Dec. 2012.

M. Karakaya, I. Korpeoglu, and O. Ulusoy. Free Riding in Peer-to-Peer Networks. IEEE Internet Computing, 13(2):92-98, Mar. 2009.

S. Kashyap, S. Deb, K. V. M. Naidu, R. Rastogi, and A. Srinivasan. Efficient GossipBased Aggregate Computation. In Proceedings of the 25th Symposium on Principles of Database Systems - PODS 2006, pages 308-317, New York, NY, USA, June 2006. ACM Press.

J. Kelso and K. Bruce. 2001 BTS Core Databook. Technical report, U.S. Department of Energy, Office of Building Technology, State and Community Programs, Washington, DC, USA, Nov. 2001. 
D. Kempe, A. Dobra, and J. Gehrke. Gossip-based Computation of Aggregate Information. In Proceedings of the 44th Annual IEEE Symposium on Foundations of Computer Science, FOCS 2003, pages 482-491, Washington, DC, USA, Oct. 2003. IEEE Computer Society.

O. Kennedy, C. Koch, and A. Demers. Dynamic Approaches to In-network Aggregation. In Proceedings of the 25th International Conference on Data Engineering, ICDE 2009, pages 1331-1334, Los Alamitos, CA, USA, Mar. 2009. IEEE.

R. Keralapura, N. Taft, and G. Iannaccone. Race Conditions in Coexisting Overlay Networks. IEEE/ACM Transactions on Networking, 16(1):1-14, Feb. 2008.

A.-M. Kermarrec and M. van Steen. Gossiping in Distributed Systems. ACM SIGOPS Operating Systems Review - Gossip-based computer networking, 41(5):2, Oct. 2007.

A. D. Keromytis, V. Misra, and D. Rubenstein. SOS: Secure Overlay Services. ACM SIGCOMM Computer Communication Review, 32(4):61-72, Oct. 2002.

H. Khurana, M. Hadley, N. Lu, and D. A. Frincke. Smart-Grid Security Issues. IEEE Security \& Privacy Magazine, 8(1):81-85, Jan. 2010.

J. K. Kok, C. J. Warmer, and I. G. Kamphuis. PowerMatcher: multiagent control in the electricity infrastructure. In Proceedings of the 4th International Joint Conference on Autonomous Agents and Multiagent Systems, AAMAS 2005, pages 75-82, New York, NY, USA, July 2005. ACM Press.

G. Koloniari and E. Pitoura. A Game-Theoretic Approach to the Formation of Clustered Overlay Networks. IEEE Transactions on Parallel and Distributed Systems, 23(4):589$597,2012$.

J. Kurian and K. Sarac. A Survey on the Design, Applications, and Enhancements of Application-Layer Overlay Networks. ACM Computing Surveys, 43(1):1-34, Nov. 2010.

J. Leitao, J. Pereira, and L. Rodrigues. Epidemic Broadcast Trees. In Proceedings of the 26th International Symposium on Reliable Distributed Systems, SRDS 2007, pages 301-310, Los Alamitos, CA, USA, Oct. 2007. IEEE.

G. Lekakos and G. M. Giaglis. A hybrid approach for improving predictive accuracy of collaborative filtering algorithms. User Modeling and User-Adapted Interaction, 17 (1-2):5-40, Jan. 2007.

B. Li, J. Guo, and M. Wang. iOverlay: A Lightweight Middleware Infrastructure for Overlay Application Implementations. In Proceedings of the 5th ACM/IFIP/USENIX International Conference on Middleware, Middleware 2004, volume 3231 of Lecture Notes in Computer Science, pages 135-154, New York, USA, Oct. 2004. SpringerVerlag New York.

C. Li, H. Wang, W. de Haan, C. J. Stam, and P. Van Mieghem. The Correlation of Metrics in Complex Networks with Applications in Functional Brain Networks. Journal of Statistical Mechanics: Theory and Experiment, 2011(11), Nov. 2011. 
F. Li, C. Pei, A. Jussara, and B. Z. Andrei. Summary Cache: A Scalable Wide-Area Web Cache Sharing Protocol. IEEE/ACM Transactions on Networking, 8(3):281-293, June 2000.

M. Li, W.-c. Lee, and A. Sivasubramaniam. DPTree: A Balanced Tree Based Indexing Framework for Peer-to-Peer Systems. In Proceedings of the International Conference on Network Protocols, ICNP 2006, pages 12-21, Los Alamitos, CA, USA, Nov. 2006. IEEE.

M. A. Lisovich, M. K. Deirdre, and S. B. Wicker. Inferring Personal Information from Demand-Response Systems. IEEE Security \& Privacy, 8(1):11-20, 2010.

H. Liu, A. Abraham, and Y. Badr. Neighbor Selection in Peer-to-Peer Overlay Networks: A Swarm Intelligence Approach. In Pervasive Computing: Innovations in Intelligent Multimedia and Applications, Computer Communications and Networks, chapter 18, pages 405-431. Springer London, London, Sept. 2010.

N. Lu, D. P. Chassin, and S. E. Widergren. Modeling Uncertainties in Aggregated Thermostatically Controlled Loads Using a State Queueing Model. IEEE Transactions on Power Systems, 20(2):725-733, May 2005.

K. Lua, J. Crowcroft, M. Pias, R. Sharma, and S. Lim. A Survey and Comparison in Peer-to-peer Overlay Network Schemes. Communications Surveys \& Tutorials, IEEE, 7(2):72 - 93, Apr. 2005.

S. P. Mahambre, M. Kumar S.D., and U. Bellur. A Taxonomy of QoS-Aware, Adaptive Event-Dissemination Middleware. IEEE Internet Computing, 11(4):35-44, July 2007.

D. Malkhi, M. Naor, and D. Ratajczak. Viceroy: A Scalable and Dynamic Emulation of the Butterfly. In Proceedings of the 21st Annual Symposium on Principles of Distributed Computing, PODC 2002, pages 183-192, New York, NY, USA, July 2002. ACM Press.

N. Manouselis and C. Costopoulou. Analysis and Classification of Multi-Criteria Recommender Systems. World Wide Web, 10(4):415-441, Mar. 2007.

Y. Mao, B. T. Loo, Z. Ives, and J. M. Smith. MOSAIC: Unified Declarative Platform for Dynamic Overlay Composition. In Proceedings of the International Conference on Emerging Networking Experiments and Technologies, CONEXT 2008, pages 1-12, New York, USA, Dec. 2008. ACM Press.

S. Massoud Amin and B. Wollenberg. Toward a smart grid: power delivery for the 21 st century. IEEE Power and Energy Magazine, 3(5):34-41, Sept. 2005.

R. Matei, A. Iamnitchi, and P. Foster. Mapping the Gnutella network. IEEE Internet Computing, 6(1):50-57, Jan. 2002.

P. E. Matheson. Epictetus: The discourses and manual, together with fragments of his writings. Clarendon Press, 1916.

P. Maymounkov and D. Mazières. Kademlia: A Peer-to-Peer Information System Based on the XOR Metric. In Proceedings of the 1st International Workshop on Peer-To-Peer Systems, IPTPS 2002, volume 2429 of Lecture Notes in Computer Science, pages 5365, Heidelberg, Oct. 2002. Springer Berlin. 
C. McDonough and R. Kraus. Does Dynamic Pricing Make Sense for Mass Market Customers? The Electricity Journal, 20(7):26-37, Aug. 2007.

A. R. Metke and R. L. Ekl. Smart Grid Security Technology. In Proceedings of the 1st Conference on Innovative Smart Grid Technologies, ISGT 2010, pages 1-7, Los Alamitos, CA, USA, Jan. 2010. IEEE.

A. Middelberg, J. Zhang, and X. Xia. An optimal control model for load shifting - With application in the energy management of a colliery. Applied Energy, 86(7-8):12661273, July 2009.

J. Mingers. Combining IS Research Methods: Towards a Pluralist Methodology. Information Systems Research, 12(3):240-259, Sept. 2001.

K. Mizutani, S. Matsuura, S. Doi, K. Fujikawa, and H. Sunahara. An Implementation and Its Evaluation of a Framework for Managing States of Nodes among Structured Overlay Networks. In Proceedings of the 6th International Conference on Networking and Services, ICNS 2010, pages 282-287, Los Alamitos, CA, USA, Mar. 2010. IEEE.

E. Modiano, D. Shah, and G. Zussman. Maximizing Throughput in Wireless Networks via Gossiping. ACM SIGMETRICS Performance Evaluation Review, 34(1):27, June 2006.

J. C. Mogul. Emergent (Mis)behavior vs. Complex Software Systems. ACM SIGOPS Operating Systems Review, 40(4):293, Oct. 2006.

J. W. Moon and S.-H. Han. Thermostat strategies impact on energy consumption in residential buildings. Energy and Buildings, 43(2-3):338-346, Feb. 2011.

J. Müller-Quade and R. Renner. Composability in quantum cryptography. New Journal of Physics, 11(8):085006, Aug. 2009.

S. K. Nabeel Ahmed, David Hadaller. Incremental Maintenance of Global Aggregates. Technical report, University of Waterloo, Waterloo, Ontario, June 2006.

K. Nahrstedt, R. Chang, and C. Ward. GoS-Assured Service Composition in Managed Service Overlay Networks. In Proceedings of the 23rd International Conference on Distributed Computing Systems, ICDCS 2003, pages 194-201, Los Alamitos, CA, USA, May 2003. IEEE.

S. Nath, P. B. Gibbons, S. Seshan, and Z. Anderson. Synopsis Diffusion for Robust Aggregation in Sensor Networks. ACM Transactions on Sensor Networks, 4(2):1-40, Mar. 2008.

A. L. Nelson, G. J. Barlow, and L. Doitsidis. Fitness functions in evolutionary robotics: A survey and analysis. Robotics and Autonomous Systems, 57(4):345-370, Apr. 2009.

M. Oey, S. van Splunter, E. Ogston, M. Warnier, and F. M. T. Brazier. A Framework for Developing Agent-Based Distributed Applications. In Proceedings of the International Conference on Web Intelligence and Intelligent Agent Technology, WI-IAT 2010, pages 470-474, Los Alamitos, CA, USA, Aug. 2010a. IEEE. 
M. Oey, M. Warnier, and F. M. T. Brazier. Security in Large-Scale Open Distributed Multi-Agent Systems. In Autonomous Agents, chapter 6, pages 107-130. Vedran Kordic (Ed.), InTech edition, June 2010b.

E. Ogston and S. A. Jarvis. Peer-to-peer aggregation techniques dissected. International Journal of Parallel, Emergent and Distributed Systems, 25(1):51-71, Feb. 2010.

E. Ogston, M. van Steen, and F. Brazier. Group Formation among Decentralized Autonomous Agents. Applied Artificial Intelligence, 18(9-10):953-970, Oct. 2004.

J. O'Madadhain, D. Fisher, P. Smyth, S. White, and Y.-B. Boey. Analysis and Visualization of Network Data using JUNG. Journal of Statistical Software, 10(2):1-35, 2005.

B. Overeinder and F. Brazier. Scalable Middleware Environment for Agent-Based Internet Applications. In Proceedings of the 7th International Conference on Applied Parallel Computing: State of the Art in Scientific Computing, PARA 2004, volume 3732 of Lecture Notes in Computer Science, pages 675-679, Heidelberg, Feb. 2006. Springer Berlin.

P. Palensky and D. Dietrich. Demand Side Management: Demand Response, Intelligent Energy Systems, and Smart Loads. IEEE Transactions on Industrial Informatics, 7(3): 381-388, Aug. 2011.

M. Paulus and F. Borggrefe. The potential of demand-side management in energyintensive industries for electricity markets in Germany. Applied Energy, 88(2):432441, Feb. 2011.

L. Ponciano and F. Brasileiro. On the Impact of Energy-saving Strategies in Opportunistic Grids. In Proceedings of the 11th IEEE/ACM International Conference on Grid Computing, Grid 2012, pages 282-289, Los Alamitos, CA, USA, Oct. 2010. IEEE.

E. Pournaras. A Survey and a Proposed Approach on Robust Tree Overlays. Technical report, VU University Amsterdam, Amsterdam, June 2009.

E. Pournaras, G. Exarchakos, and N. Antonopoulos. Load-driven neighbourhood reconfiguration of Gnutella overlay. Computer Communications, 31(13):3030-3039, Aug. 2008a.

E. Pournaras, M. Warnier, and F. M. T. Brazier. Using intelligent agents for selfadaptation and self-optimization of energy consumption in power networks. In Proceeding of the International Workshop on Agents for Autonomic Computing, AAC 2008, Chicago, IL, USA, June 2008b.

E. Pournaras, M. Warnier, F. M. T. Brazier, and E. Ogston. Towards Adaptive Energy Plan Aggregation over a Peer-to-Peer Tree Overlay. In Proceedings of the International Workshop on Peer-to-Peer Systems, P4P2P 2008, Warwick, UK, May 2008c.

E. Pournaras, M. Warnier, and F. M. T. Brazier. Adaptive Agent-Based Self-Organization for Robust Hierarchical Topologies. In Proceeding of the International Conference on Adaptive and Intelligent Systems, ICAIS 2009, pages 69-76, Los Alamitos, CA, USA, Sept. 2009a. IEEE. 
E. Pournaras, M. Warnier, and F. M. T. Brazier. A Distributed Agent-based Approach to Stabilization of Global Resource Utilization. In Proceedings of the International Conference on Complex, Intelligent and Software Intensive Systems, CISIS 2009, pages 185-192. IEEE, Mar. 2009b.

E. Pournaras, M. Warnier, and F. M. T. Brazier. Adaptation Strategies for Selfmanagement of Tree Overlay Networks. In Proceedings of the 11th IEEE/ACM International Conference on Grid Computing, Grid 2010, pages 401-409, Los Alamitos, CA, USA, Oct. 2010a. IEEE.

E. Pournaras, M. Warnier, and F. M. T. Brazier. Self-Optimised Tree Overlays Using Proximity-Driven Self-Organised Agents. In Complex Intelligent Systems and their Applications, volume 41 of Springer Optimization and its Applications, chapter 7, pages 137-161. Springer New York, New York, NY, Aug. 2010b.

E. Pournaras, M. Warnier, and F. M. T. Brazier. Local Agent-based Self-stabilisation in Global Resource Utilisation. International Journal of Autonomic Computing, 1(4):350 - 373, Dec. 2010c.

E. Pournaras, M. Warnier, and F. M. T. Brazier. Towards Emergent Energy Synchronization using Agents. In Proceedings of the 1st International Workshop on Agent Technologies for Energy Systems, ATES 2010, Toronto, Canada, May $2010 d$.

E. Pournaras, M. Yao, R. Ambrosio, and M. Warnier. Organizational Control Reconfigurations for a Robust Smart Power Grid. In Internet of Things and Inter-cooperative Computational Technologies for Collective Intelligence, volume 460 of Studies in Computational Intelligence, chapter 8, pages 189-206. Springer-Verlag, Jan. 2012.

E. Pournaras, M. Warnier, and F. M. T. Brazier. Adaptive Decentralized Aggregation. 2013a. (submitted journal).

E. Pournaras, M. Warnier, and F. M. T. Brazier. Peer-to-peer Aggregation for Dynamic Adjustments in Power Demand. 2013b. (submitted journal).

C. Prehofer and C. Bettstetter. Self-Organization in Communication Networks: Principles and Design Paradigms. IEEE Communications Magazine, 43(7):78-85, July 2005.

Y. Qiao and F. E. Bustamante. Structured and unstructured overlays under the microscope: A measurement-based view of two P2P systems that people use. In Proceedings of the Annual Technical Conference of USENIX 2006, pages 341-355, Berkeley, CA, USA, May 2006. USENIX Association.

R. Rajagopalan and P. Varshney. Data-aggregation Techniques in Sensor Networks: A Survey. IEEE Communications Surveys \& Tutorials, 8(4):48-63, Jan. 2006.

S. Ratnasamy, P. Francis, M. Handley, R. Karp, and S. Schenker. A Scalable ContentAddressable Network. In Proceedings of the International Conference on Applications, Technologies, Architectures, and Protocols for Computer Communications, SIGCOMM 2001, volume 31, pages 161-172, New York, NY, USA, Aug. 2001. ACM Press.

H. J. Richardson. A 'smart house' is not a home: The domestication of ICTs. Information Systems Frontiers, 11(5):599-608, July 2008. 
J. L. Rodgers and W. A. Nicewander. Thirteen Ways to Look at the Correlation Coefficient. The American Statistician, 42(1):59, Feb. 1988.

A. Rodriguez, C. Killian, S. Bhat, D. Kostić, and A. Vahdat. MACEDON: Methodology for Automatically Creating, Evaluating, and Designing Overlay Networks. In Proceedings of the 1st International Conference on Networked Systems Design and Implementation, NSDI 2004, pages 267-280, Berkeley, CA, USA, Mar. 2004. USENIX Association.

A. Rowstron and P. Druschel. Pastry: Scalable, Decentralized Object Location, and Routing for Large-Scale Peer-to-Peer Systems. In Proceedings of the International Conference on Distributed Systems Platforms, Middleware 2001, volume 2218 of Lecture Notes in Computer Science, pages 329-350, Heidelberg, Oct. 2001. Springer Berlin.

J. Rybicki, B. Scheuermann, W. Kiess, C. Lochert, P. Fallahi, and M. Mauve. Challenge: Peers on Wheels - A Road to New Traffic Information Systems. In Proceedings of the 13th Annual International Conference on Mobile Computing and Networking, MobiCom 2007, pages 215-221, New York, NY, USA, Sept. 2007. ACM Press.

J. Sacha. Exploiting Heterogeneity in Peer-to-Peer Systems Using Gradient Topologies. Phd thesis, University of Dublin, Trinity College, July 2009.

Y. Sang, H. Shen, Y. Inoguchi, Y. Tan, and N. Xiong. Secure Data Aggregation in Wireless Sensor Networks: A Survey. In Proceedings of the 7th International Conference on Parallel and Distributed Computing, Applications and Technologies, PDCAT 2006, pages 315-320, Los Alamitos, CA, USA, Dec. 2006. IEEE.

P. Scerri, D. V. Pynadath, and M. Tambe. Towards Adjustable Autonomy for the Real World. Journal of Artificial Intelligence Research, 17(1):171-228, Feb. 2002.

S. Schmid and R. Wattenhofer. Structuring Unstructured Peer-to-Peer Networks. In Proceedings of the 14th International Conference on High Performance Computing, HiPC 2007, pages 432-442, Heidelberg, Dec. 2007. Springer-Verlag Berlin.

I. Scholtes and C. J. Tessone. Organic Design of Massively Distributed Systems: A Complex Networks Perspective. Informatik-Spektrum, 35(2):75-86, Feb. 2012.

F. Schweppe, B. Daryanian, and R. Tabors. Algorithms for a spot price responding residential load controller. IEEE Transactions on Power Systems, 4(2):507-516, May 1989.

R. Shaw, M. Attree, T. Jackson, and M. Kay. The value of reducing distribution losses by domestic load-shifting: a network perspective. Energy Policy, 37(8):3159-3167, Aug. 2009.

A. Singh and M. Haahr. Decentralized Clustering In Pure P2P Overlay Networks Using Schelling's Model. In Proceedings of the International Conference on Communications, ICC 2007, pages 1860-1866. IEEE, June 2007.

D. I. K. Sjoberg, T. Dyba, and M. Jorgensen. The Future of Empirical Methods in Software Engineering Research. In Future of Software Engineering, FOSE 2007, pages 358-378, Los Alamitos, CA, USA, May 2007. IEEE. 
J. Spohrer, P. Maglio, J. Bailey, and D. Gruhl. Steps Toward a Science of Service Systems. Computer, 40(1):71-77, Jan. 2007.

M. Stadler, W. Krause, M. Sonnenschein, and U. Vogel. Modelling and evaluation of control schemes for enhancing load shift of electricity demand for cooling devices. Environmental Modelling \& Software, 24(2):285-295, Feb. 2009.

N. Stodola and V. Modi. Penetration of solar power without storage. Energy Policy, 37 (11):4730-4736, Nov. 2009.

I. Stoica, R. Morris, D. Liben-Nowell, D. Karger, M. Kaashoek, F. Dabek, and H. Balakrishnan. Chord: a scalable peer-to-peer lookup protocol for internet applications. IEEE/ACM Transactions on Networking, 11(1):17-32, Feb. 2003.

G. Strbac. Demand side management: Benefits and challenges. Energy Policy, 36(12): 4419-4426, Dec. 2008.

Y. Strengers. Comfort expectations: the impact of demand-management strategies in Australia. Building Research \& Information, 36(4):381-391, Aug. 2008.

D. Stutzbach, R. Rejaie, and S. Sen. Characterizing Unstructured Overlay Topologies in Modern P2P File-Sharing Systems. IEEE/ACM Transactions on Networking, 16(2): 267-280, Apr. 2008.

V. Sundramoorthy, G. Cooper, N. Linge, and Q. Liu. Domesticating Energy-Monitoring Systems: Challenges and Design Concerns. IEEE Pervasive Computing, 10(1):20-27, Jan. 2011.

G. Tan, S. A. Jarvis, X. Chen, D. P. Spooner, and G. R. Nudd. Performance Analysis and Improvement of Overlay Construction for Peer-to-Peer Live Media Streaming. Simulation, 82(2):169-178, Sept. 2005a.

S.-W. Tan, G. Waters, and J. Crawford. MeshTree: Reliable Low Delay Degree-bounded Multicast Overlays. In Proceedings of the 11th International Conference on Parallel and Distributed Systems, ICPADS 2005, volume 2, pages 565-569, Los Alamitos, CA, USA, 2005b. IEEE.

A. S. Tanenbaum and M. Van Steen. Distributed Systems: Principles and Paradigms. Pearson Prentice Hall, 2 edition, 2007.

C. Tang, R. Chang, and C. Ward. GoCast: Gossip-Enhanced Overlay Multicast for Fast and Dependable Group Communication. In Proceedings of the International Conference on Dependable Systems and Networks, DSN 2005, pages 140-149, Los Alamitos, CA, USA, 2005. IEEE.

S. Tang, E. Jaho, I. Stavrakakis, I. Koukoutsidis, and P. V. Mieghem. Modeling gossipbased content dissemination and search in distributed networking. Computer Communications, 34(6):765-779, May 2011.

C. Taylor. Reporter in Red China. Random House, 1966.

J. Turner and D. Taylor. Diversifying the Internet. In Proceedings of the Global Telecommunications Conference, GLOBECOM 2005, pages 755-760, Los Alamitos, CA, USA, Dec. 2005. IEEE. 
P. Van Mieghem, J. Omic, and R. Kooij. Virus spread in networks. IEEE/ACM Transactions on Networking, 17(1):1-14, Feb. 2009.

R. Van Renesse, K. P. Birman, and W. Vogels. Astrolabe: A Robust and Scalable Technology For Distributed System Monitoring, Management, and Data Mining. ACM Transactions on Computer Systems, 21(2):164-206, May 2003.

C. Vassilakis and I. Stavrakakis. Minimizing node churn in peer-to-peer streaming. Computer Communications, 33(14):1598-1614, Sept. 2010.

J. Vidal, P. Buhler, and M. Huhns. Inside an agent. Internet Computing, IEEE, 5(1): 82-86, Jan. 2001.

V. Vishnumurthy and P. Francis. On Heterogeneous Overlay Construction and Random Node Selection in Unstructured P2P Networks. In Proceedings of the 25th International Conference on Computer Communications, INFOCOM 2006, pages 1-12, Los Alamitos, CA, USA, 2006. IEEE.

A. Walters, D. Zage, and C. N. Rotaru. A Framework for Mitigating Attacks Against Measurement-Based Adaptation Mechanisms in Unstructured Multicast Overlay Networks. IEEE/ACM Transactions on Networking (TON), 16(6):1434-1446, Dec. 2008.

F. Wang, Y. Xiong, and J. Liu. mTreebone: A Collaborative Tree-Mesh Overlay Network for Multicast Video Streaming. IEEE Transactions on Parallel and Distributed Systems, 21(3):379-392, Mar. 2010.

X. Wang, Y. L. Yin, and H. Yu. Finding Collisions in the Full SHA-1. In Proceedings of the 25th Annual International Cryptology Conference, CRYPTO 2005, volume 3621 of Lecture Notes in Computer Science, pages 16-36, Heidelberg, Aug. 2005. Springer Berlin.

Y. Wang and M. P. Singh. Trust Representation and Aggregation in a Distributed Agent System. In Proceedings of the 21st National Conference on Artificial Intelligence, AAAI 2006, volume 2, pages 1425-1430, Boston, Massachusetts, July 2006. AAAI Press.

T. Weise, M. Zapf, and K. Geihs. Evolving Proactive Aggregation Protocols. In Proceedings of the 11th European Conference on Genetic Programming, EuroGP 2008, volume 4971 of Lecture Notes in Computer Science, pages 254-265, Heidelberg, Apr. 2008. Springer-Verlag Berlin.

B. Yang and H. Garcia-Molina. Designing a Super-Peer Network. In Proceedings of the 19th International Conference on Data Engineering, ICDE 2003, pages 49-60, Los Alamitos, CA, USA, Mar. 2003. IEEE Computer Society.

M. Yang and Z. Fei. A cooperative failure detection mechanism for overlay multicast. Journal of Parallel and Distributed Computing, 67(6):635-647, June 2007.

Y. Yang, R. Dunlap, M. Rexroad, and B. F. Cooper. Performance of Full Text Search in Structured and Unstructured Peer-to-Peer Systems. In Proceedings of the 25th International Conference on Computer Communications, INFOCOM 2006, pages 1-12, Los Alamitos, CA, USA, 2006. IEEE. 
L. Yuan and O. Wei Tsang. Distributed Construction of Resource-efficient Overlay Tree by Approximating MST. In Proceedings of the International Conference on Multimedia and Expo, ICME 2004, pages 1507-1510, Los Alamitos, CA, USA, 2004. IEEE.

J. Yuh-Jzer, F. Chien-Tse, and Y. Li-Wei. Keyword Search in DHT-Based Peer-to-Peer Networks. In Proceedings of the 25th IEEE International Conference on Distributed Computing Systems, ICDCS 2005, pages 339-348, Los Alamitos, CA, USA, Jan. 2005. IEEE.

E. Zeller and S. F. Alleyne. A History of Greek Philosophy - From the Earliest Period to the Time of Socrates, volume II. Longmans, Green, London, 1881.

G. Zhang, B. L. Smith, and J. Guo. Peer-to-Peer-Based Publish/Subscribe Architecture for Advanced Infrastructure Systems. Journal of Computing in Civil Engineering, 24 (1):65, Dec. 2010.

B. Zhao, L. Huang, J. Stribling, S. Rhea, A. Joseph, and J. Kubiatowicz. Tapestry: A Resilient Global-Scale Overlay for Service Deployment. IEEE Journal on Selected Areas in Communications, 22(1):41-53, Jan. 2004.

B. Y. Zhao, Y. Duan, L. Huang, A. D. Joseph, and J. Kubiatowicz. Brocade: Landmark Routing on Overlay Networks. In Proceedings of the 1st International Workshop on Peer-to-Peer Systems, IPTPS 2001, pages 34-44, London, UK, Mar. 2002. SpringerVerlag.

J. Zhao, F. Yang, Q. Zhang, Z. Zhang, and F. Zhang. LION: Layered Overlay Multicast With Network Coding. IEEE Transactions on Multimedia, 8(5):1021-1032, Oct. 2006.

D. Zhenhai, Z. Zhi-Li, and Y. Hou. Service Overlay Networks: SLAs, QoS and Bandwidth Provisioning. IEEE/ACM Transactions on Networking, 11(6):870-883, Dec. 2003.

H. Zhuge and L. Feng. Distributed Suffix Tree Overlay for Peer-to-Peer Search. IEEE Transactions on Knowledge and Data Engineering, 20(2):276-285, Feb. 2008. 



\section{Appendix A

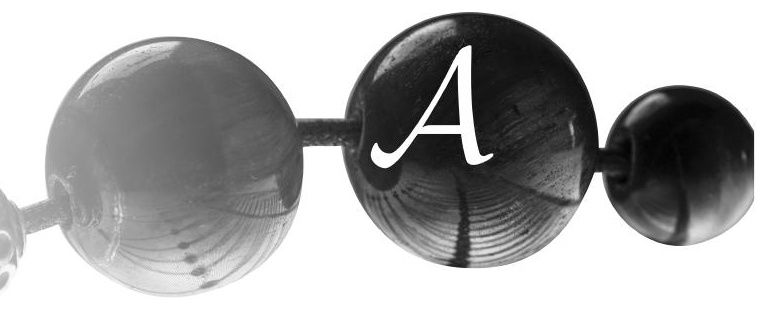

\section{Supplemental Material of Chapter 4}

Assume a ranked node $w_{i}$ that has in its tree view $\mathbf{v}_{i}($ tree $)$ the parent $w_{p}$ and the ordered $(>)$ set of children $\left\{w_{c_{\uparrow}}, \ldots, w_{c_{+}}\right\}$. This node performs coordination with a ranked node $w_{j}$ using horizontal interactions of ASMA. The request and removal messages are used as outgoing criteria and the acknowledgment and rejection messages as outgoing samples. In both cases, the weights of the source and destination are included using the getCriteria and get Samples subtasks. Algorithm A.1 and A.2 illustrate the peer-to-peer coordination between the ranked nodes $w_{i}$ and $w_{j}$ based on the initializeCoordination and finalizeCoordination subtasks. These subtasks are used by Algorithm 3.14 and 3.13 of the ASMA architecture respectively.

In the initializeCoordination subtask, a request is potentially sent to a candidate parent or child $w_{j}$ provided by the structuring level. This request is actually sent if and only if this candidate parent/child either fills the tree view or updates it by replacing a lower ranked node (line 2 and 9 of Algorithm A.1).

Coordination completes with the execution of the finalizeCoordination subtask. The response of a node to a request depends on the feedback that the generated structuring criteria define. Positive feedback corresponds to an acknowledgment message and negative feedback corresponds to a rejection message.

The organize subtask facilitates the coordination logic by reacting to the received messages. It is used in Algorithm 3.14 of the ASMA architecture and is executed as shown in Algorithm A.3. The rest of the algorithms illustrate the reactions to each of the received messages.

Algorithm A.4 outlines the reaction to a received parent request. There are two conditions under which a parent-child link is established. In the first condition (line 1 of Algorithm A.4), the tree view of the node $w_{i}$ is not full. In the second condition (line 2 of Algorithm A.4), the lowest ranked child $c_{\dashv}$ is replaced with the ranked node $w_{j}$ (lines 3-9 of Algorithm A.4) if it is ranked lower than $w_{j}$. The link establishment follows an acknowledgment message (lines 7-8 of Algorithm A.4), whereas in case none of these conditions are met, a rejection message (lines 14-15 of Algorithm A.4). Note that in case of an acknowledgment, the structuring criteria are built containing positive feedback and the weight $w_{j}$ of the new child (lines 10-12 of Algorithm A.4). 


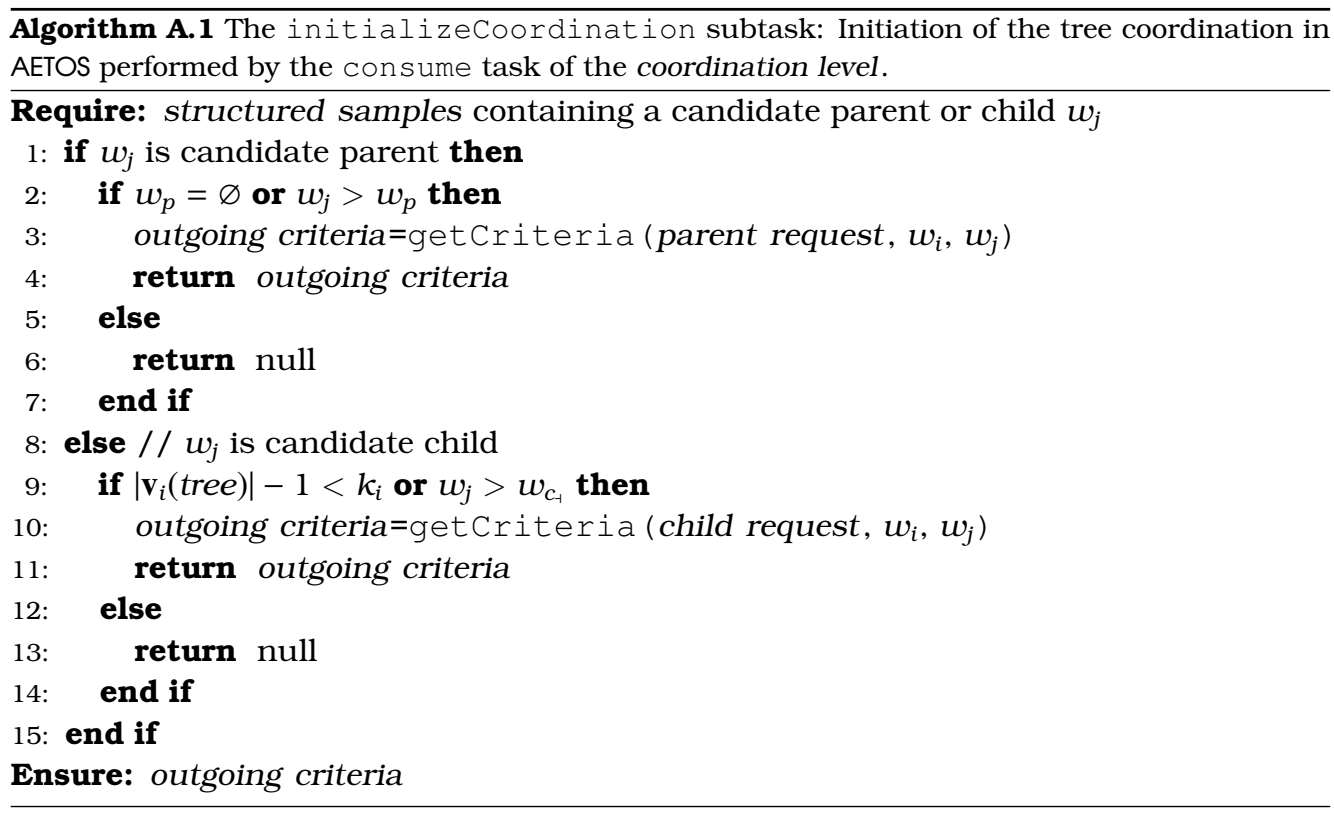

Algorithm A.2 The finalizeCoordination subtask: Completion of the tree coordination in AETOS performed by the adapt task of the coordination level.

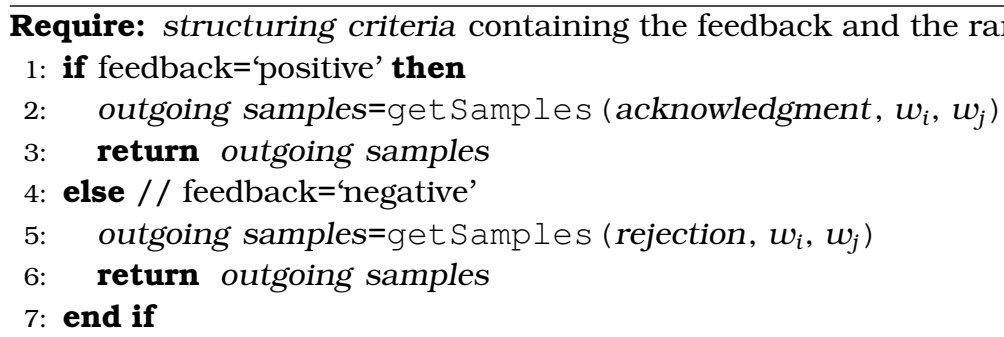

Ensure: outgoing samples

Algorithm A.5 illustrates the reactions to a received child request message that follow the same concept to the reactions of a received parent request.

Algorithm A.6 illustrates the reactions to a received acknowledgment message that results in the establishment of a new parent-child link. The sender node $w_{j}$, as a new parent or child, is added in the tree view $\mathbf{v}_{i}$ (tree) of node $w_{i}$ (line 7 and 17 of Algorithm A.6). A potential removal message is sent if a node is replaced by $w_{j}$ (lines 3-4 and 13-14 of Algorithm A.6). A positive feedback and the new ranked node added in the tree view form the structuring criteria for adapting the structuring level of AETOS (lines 8-9 and 18-19 of Algorithm A.6).

The reactions to a received rejection message do not introduce any changes in the tree view as illustrated in Algorithm A.7. However, a rejection message triggers structuring criteria containing a negative feedback and the weight of the sender node $w_{j}$.

Finally, the Algorithm A.8 shows that a ranked node $w_{j}$ is removed from the tree view if a removal message is received. A removal of a node from the tree view is always 


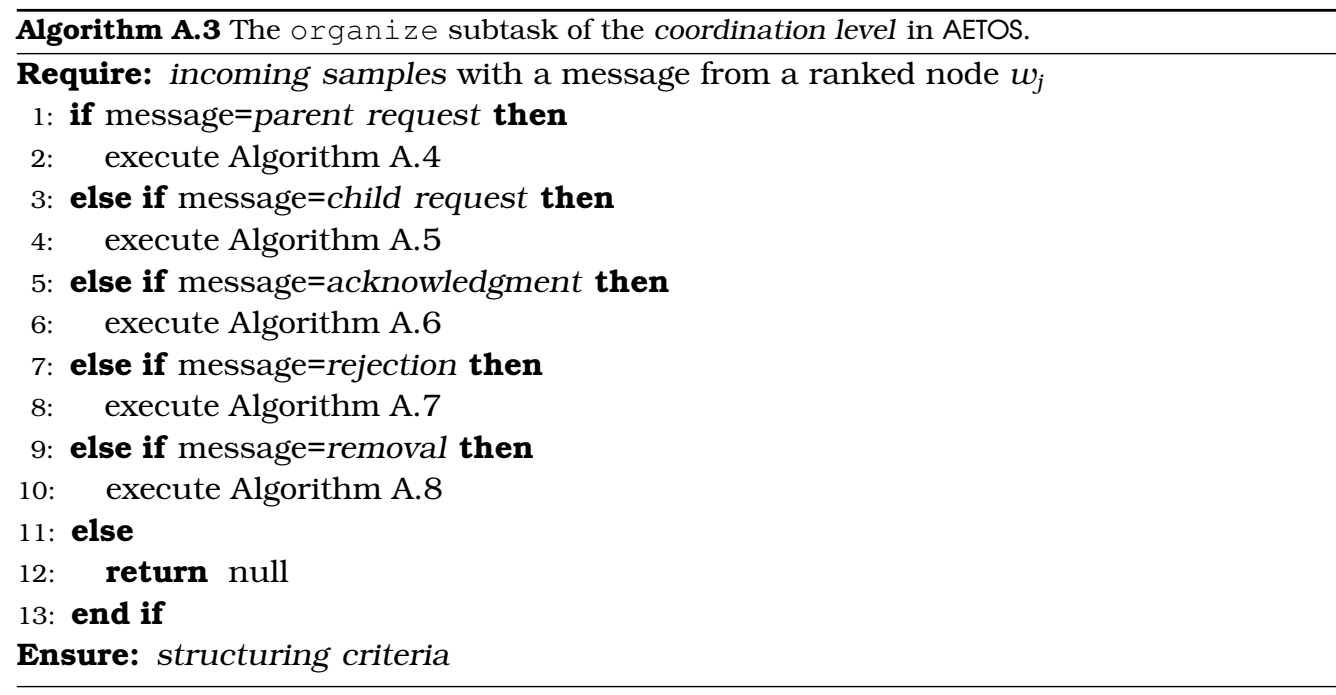

Algorithm A.4 The reactions to a parent request message received in the coordination level of AETOS.

Require: parent request message from a ranked node $w_{j}$

1: if $\mid \mathbf{v}_{i}$ (tree) $\mid-1<k_{i}$ or $w_{j}>w_{c_{\dashv}}$ then

2: $\quad$ if $\mid \mathbf{v}_{i}($ tree $) \mid-1=k_{i}$ and $w_{j}>w_{c_{+}}$then

3: $\quad$ outgoing criteria=getCriteria (removal, $w_{i}, w_{c_{-}}$)

4: $\quad$ configure (outgoing criteria)

5: $\quad\left\{w_{c_{\uparrow}}, \ldots, w_{c_{\uparrow}}\right\} \backslash w_{c_{\uparrow}}$

6: end if

7: $\quad$ outgoing samples=get Samples (acknowledgment, $\left.w_{i}, w_{j}\right)$

8: provide (outgoing samples)

9: $\quad\left\{w_{c_{\vdash}}, \ldots, w_{c_{\uparrow}}\right\} \cup w_{j}$

10: feedback='positive'

11: $\quad$ structuring criteria $=$ get Criteria (feedback, $w_{c_{-}}$)

12: return structuring criteria

13: else

14: $\quad$ outgoing samples=get Samples (rejection, $\left.w_{i}, w_{j}\right)$

15: provide (outgoing samples)

16: return null

17: end if

Ensure: structuring criteria

accompanied with structuring criteria containing a negative feedback.

The nodes of the tree view are the actual organized samples provided to the application. The condition of delivery (line 1 of Algorithm 3.11) is controlled by the organizational criteria provided by the application. Examples of criteria that can be engaged are a periodic delivery or a minimum rate of changes occurring in the tree view.

Finally, note that the coordination performed in the coordination level assumes a synchronous communication for the matters of simplicity in the illustration of the 


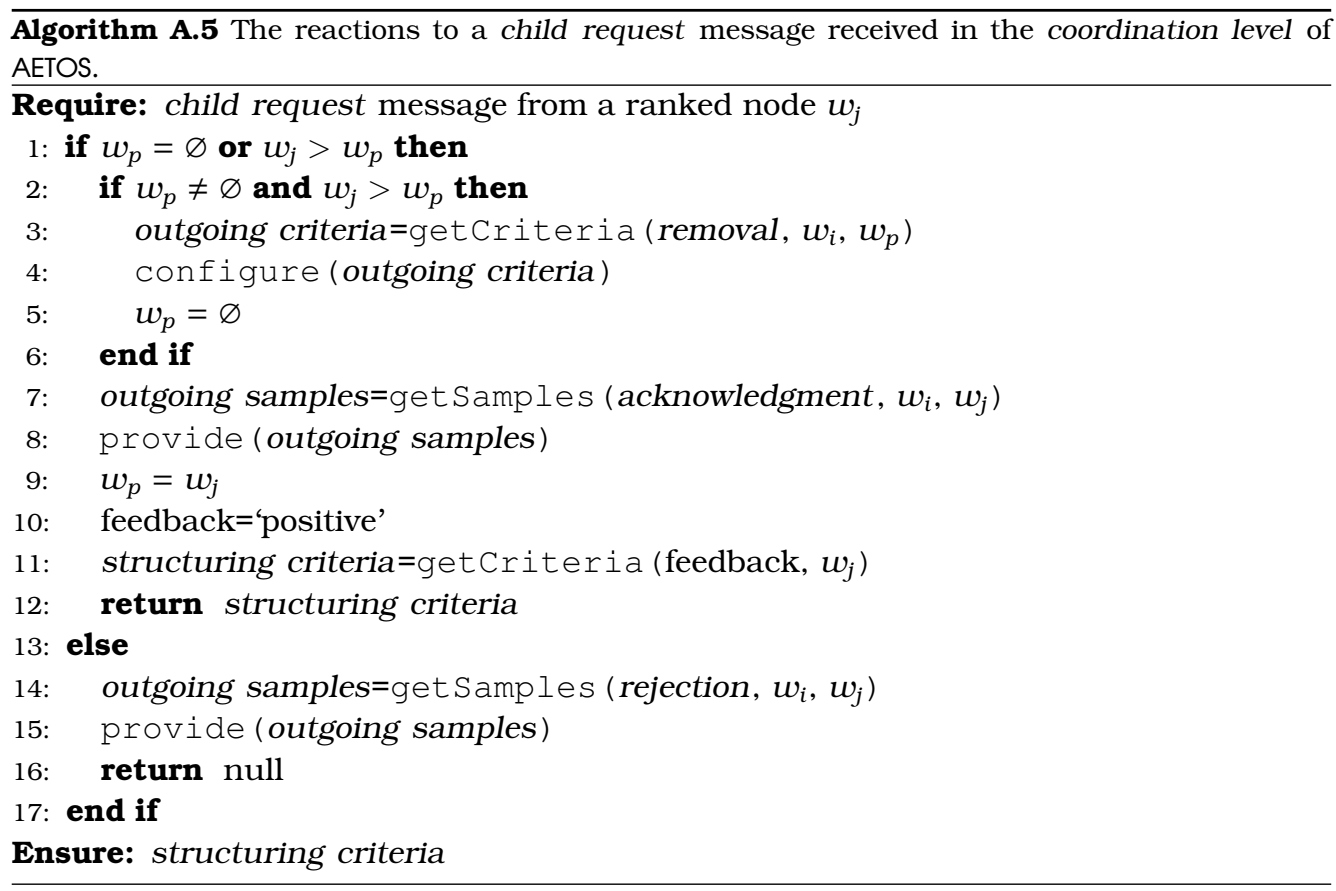

algorithms. Nonetheless, a concurrent version of the coordination level in AETOS is implemented in the simulation framework of AgentScape. In contrast to the coordination level, the gossiping realizations of the discovery level and structuring level tolerate the lack of concurrency with various solutions discussed by Jelasity et al. [2007]. 


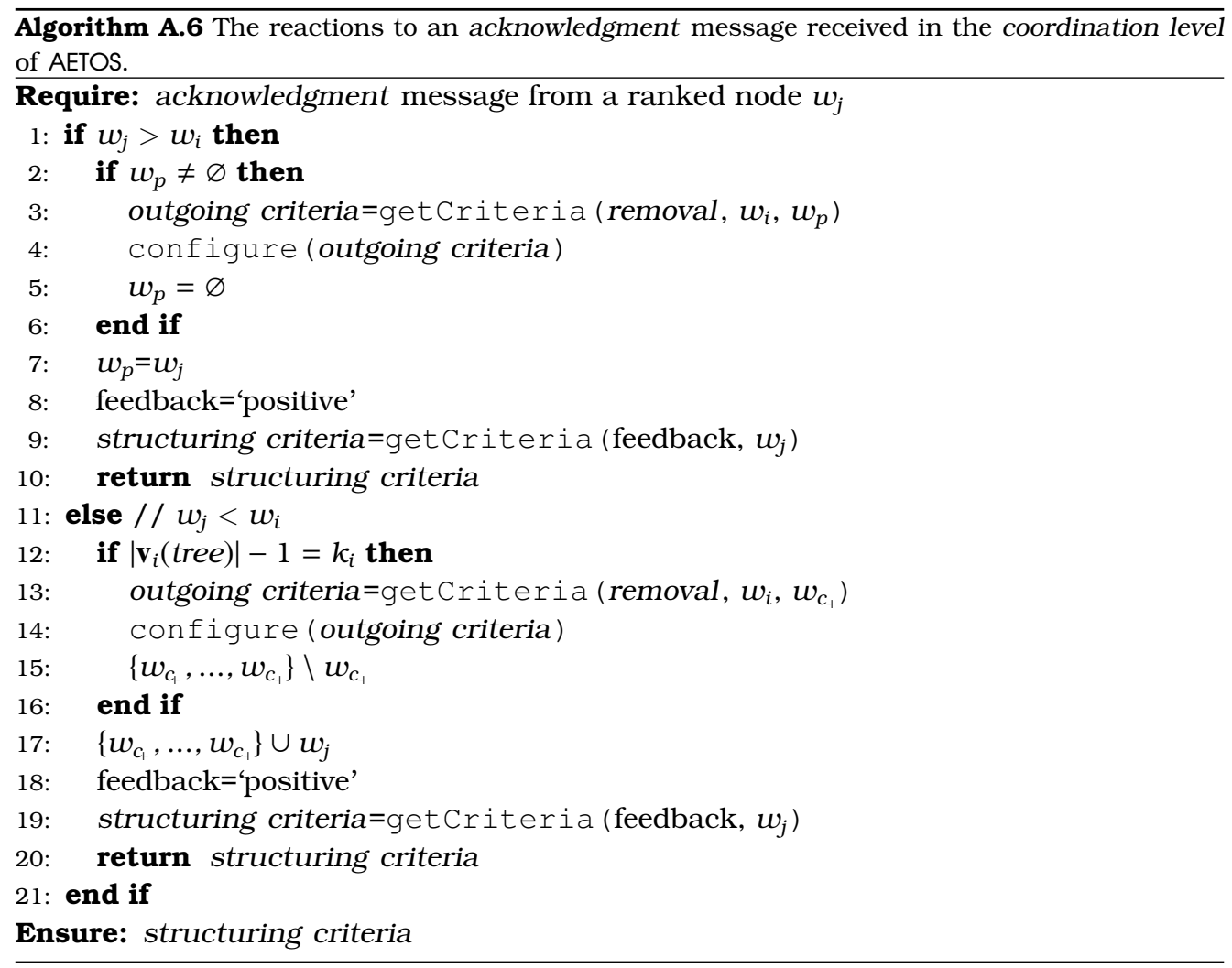

Algorithm A.7 The reactions to a rejection message received in the coordination level of AETOS.

Require: rejection message from a ranked node $w_{j}$

1: feedback='negative'

2: structuring criteria=getCriteria (feedback, $w_{j}$ )

3: return structuring criteria

Ensure: structuring criteria

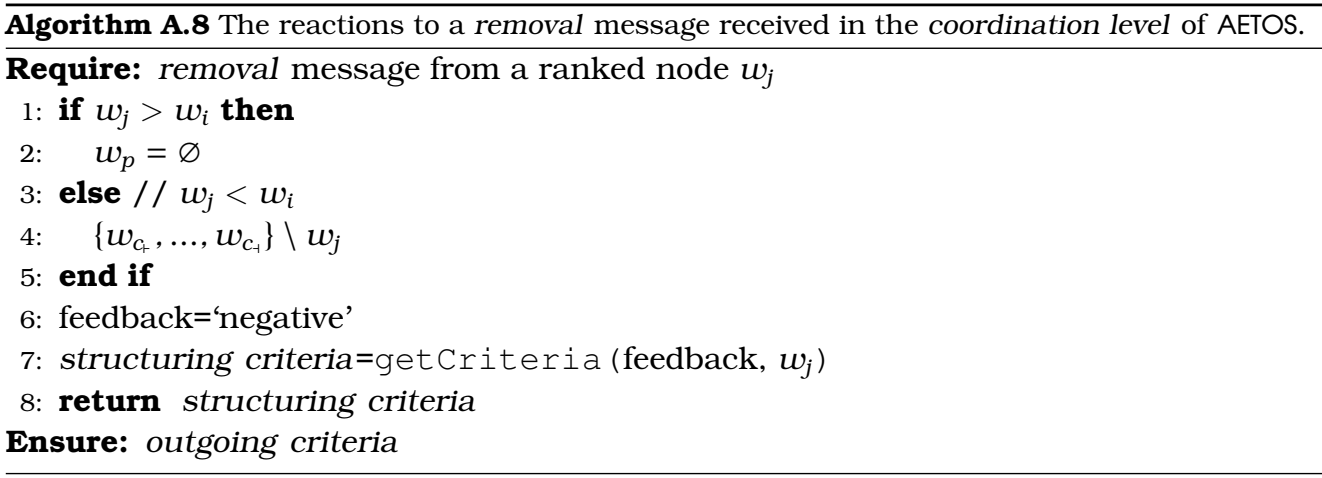





\section{Appendix B}

\section{Supplemental Material of Chapter 5}

Figure B. 1 shows the five beta distributions ${ }^{1}$ used for the parameterization of the possible states in the experimental evaluation of DIAS.

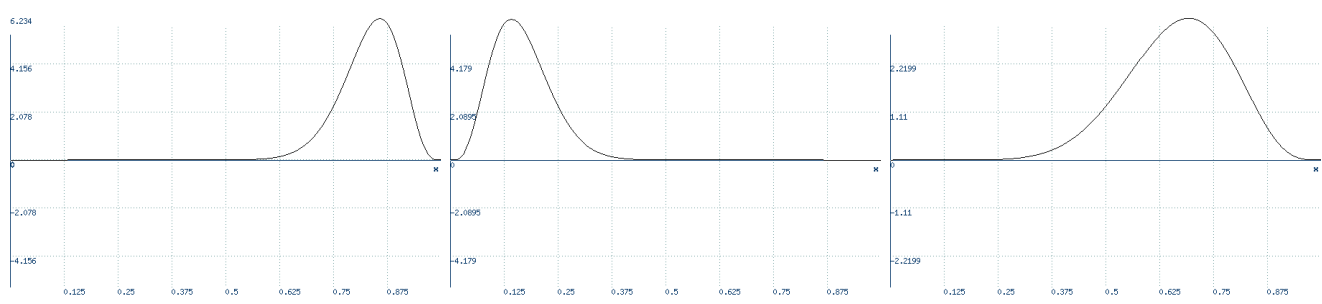

(a) alpha $=25$, beta $=5$.

(b) alpha $=5$, beta $=25$.

(c) alpha $=10$, beta $=5$.

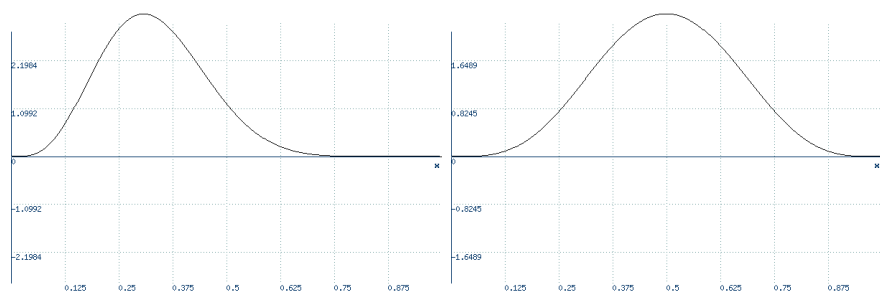

(d) alpha $=5$, beta $=10$.

(e) alpha $=5$, beta $=5$.

Figure B.1: Five beta distribution used as the input domain of the possible states. In each aggregation epoch, a random value from a given beta distribution is assigned to a possible state.

Figure B.2, B.3, B.4 and B.5 illustrate the source data of the experimental results based on which the accuracy and matching are computed.

\footnotetext{
${ }^{1}$ Generated by the Wessa online statistics software, available at: http: / /www. wessa. net/ (Last accessed: January 2013)
} 


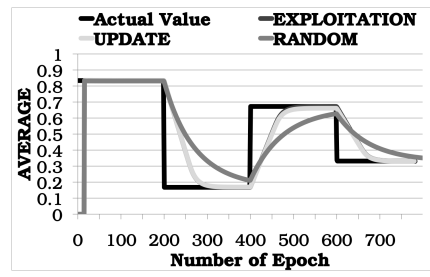

(a) Average.

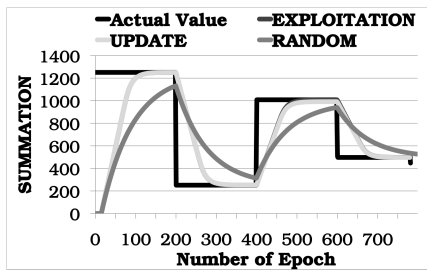

(b) Summation.

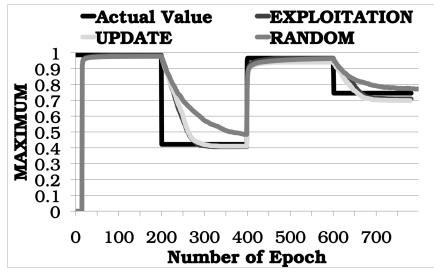

(c) Maхimum.

Figure B.2: Convergence of aggregates under synchronous changes for the EXPLOITATION, UPDATE and RANDOM strategies.

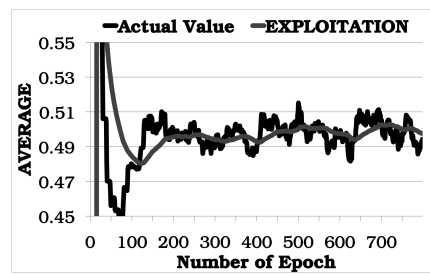

(a) Exploitation.

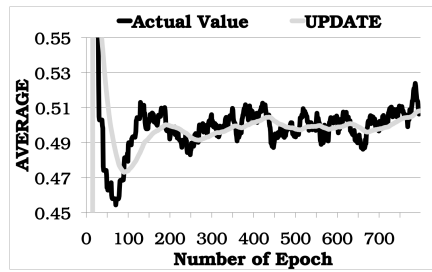

(b) UPDATE.

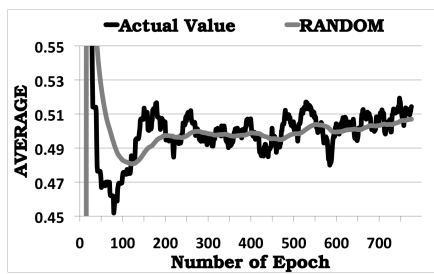

(c) RANDOM.

Figure B.3: Convergence of AVERAGE for the adaptation strategies under asynchronous changes.

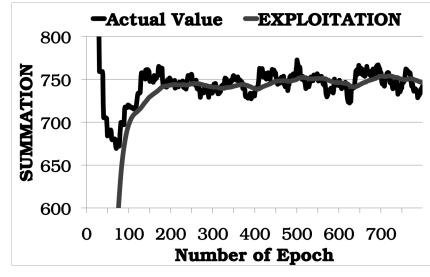

(a) Exploitation.

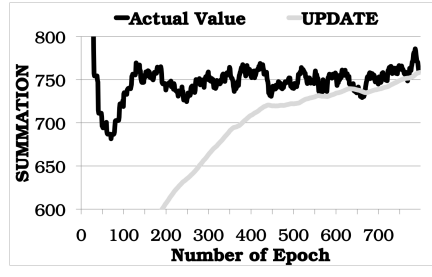

(b) UPDATE.

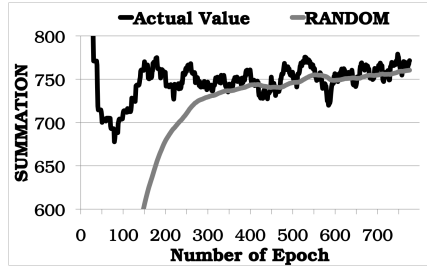

(c) RANDOM.

Figure B.4: Convergence of summation for the adaptation strategies under asynchronous changes.

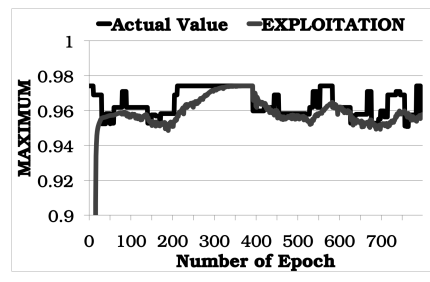

(a) Exploitation.

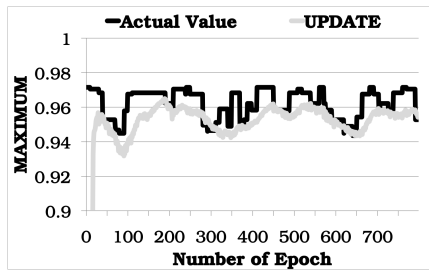

(b) UPDATE.

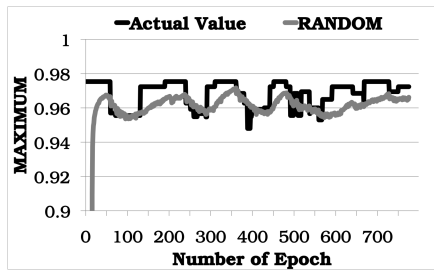

(c) RANDOM.

Figure B.5: Convergence of мАХІмUM for the adaptation strategies under asynchronous changes. 


\section{Appendix C}

\section{Supplemental Material of Chapter 6}

Figure C.1 illustrates the database schema of the data collected during the year of the Olympic Peninsula Project. The data used in Chapter 6 mainly concern the tables cust_tstat_trans and billing that contain information about the power demand and the contracts of consumers respectively during the project year. 


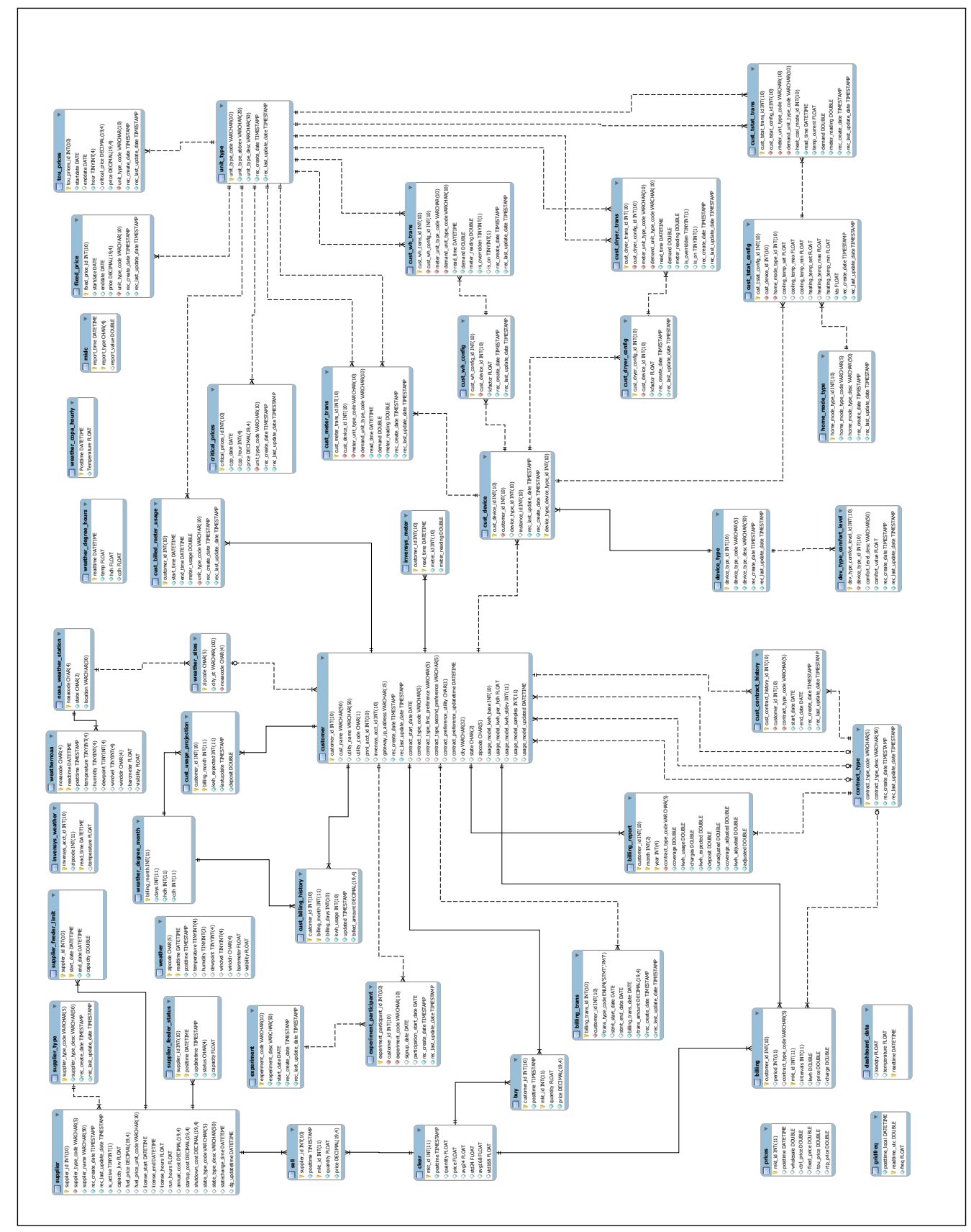

Figure C.1: The database schema of the Olympic Peninsula Project [Hammerstrom, 2007]. 


\section{List of Symbols}

$n \quad$ The number of nodes in an overlay network. $\ldots \ldots \ldots \ldots \ldots \ldots \ldots \ldots \ldots$

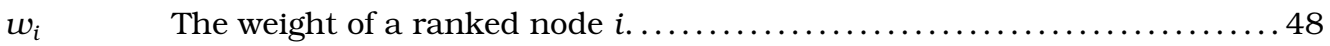

d The maximum number of overlay links that a node can establish.......48

$k \quad$ The maximum number of children in a tree view. $\ldots \ldots \ldots \ldots \ldots \ldots \ldots . \ldots 48$

$\mathbf{v}_{i}(x) \quad$ The links of an overlay network $x$ managed by a node $i \ldots \ldots \ldots \ldots \ldots 48$

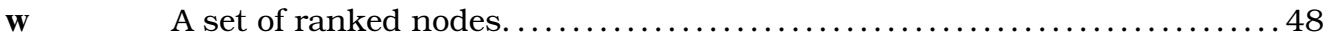

k A set with the maximum number of children for each node of a tree. ... 48

T A superset containing the levels of a tree as sets of nodes............49

$h \quad$ The number of levels (height) of a tree ........................ 49

$p_{\vdash} \quad$ The highest ranked node in a set of parents...................... 49

$p_{\dashv} \quad$ The lowest ranked node in a set of parents....................... 49

$c_{\vdash} \quad$ The highest ranked node of a set of children. $\ldots \ldots \ldots \ldots \ldots \ldots \ldots \ldots \ldots$

$c_{\dashv} \quad$ The lowest ranked node in a set of children. ..................... 49

$g_{u} \quad$ The number of nodes in the $u$ level of a tree....................... 49

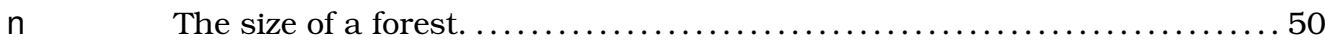

$\beta \quad$ The performance metric of connectedness in AETOS.................50

$\gamma \quad$ The performance metric of connectivity in AETOS.....................50

$\rho \quad$ The performance metric of fitness in AETOS........................ 50

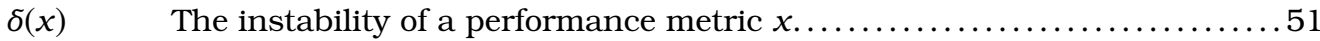

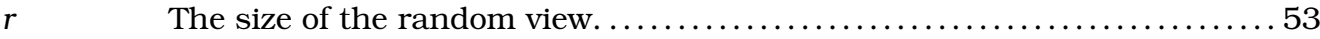

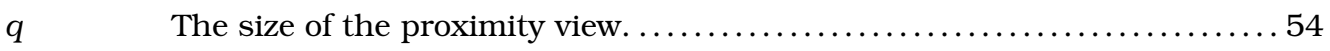

$f \quad$ The scaling factor that controls the size of the proximity view. .........54

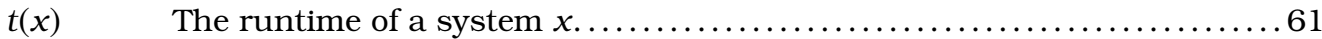


$t^{\prime}(x) \quad$ The bootstrapping time period of a system $x \ldots \ldots \ldots \ldots \ldots \ldots \ldots \ldots \ldots \ldots \ldots \ldots \ldots$

$z \quad$ The number of events generation.

$m$

The size of the T-MAN view.

The number of elements exchanged in T-MAN. .................. 62

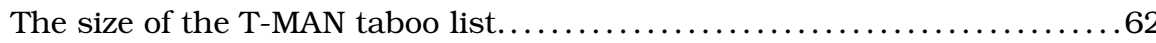

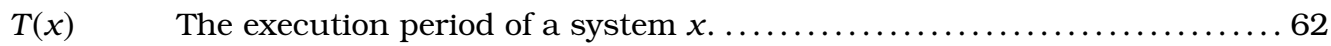

$\lambda(x) \quad$ The number of messages generated by a system $x \ldots \ldots \ldots \ldots \ldots \ldots \ldots \ldots$

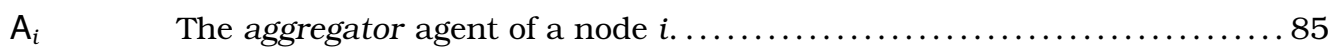

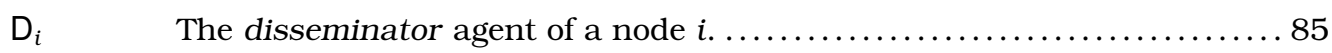

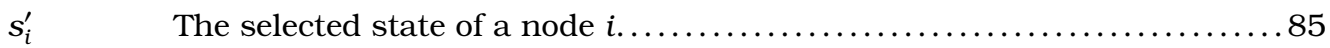

$v$

The number of possible states............................. 85

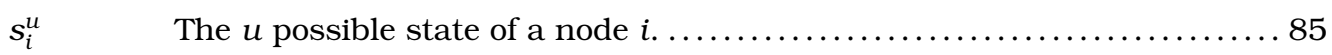

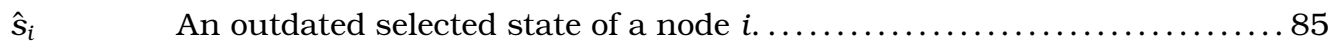

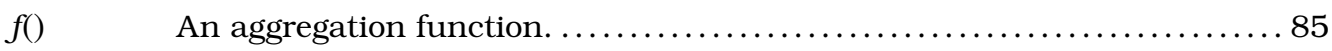

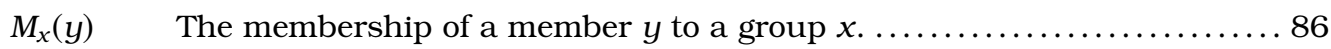

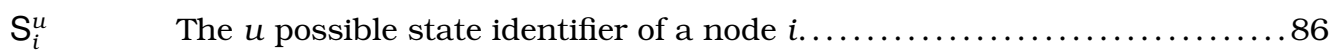

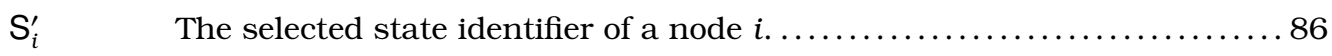

$\hat{\mathrm{S}}_{i} \quad$ An outdated selected state identifier of a node $i \ldots \ldots \ldots \ldots \ldots \ldots \ldots \ldots$

k The number of hash functions of a bloom filter................... 93

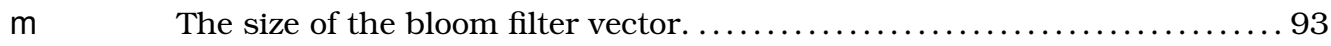

$P_{f p}(x) \quad$ The false positive probability of a bloom filter $x \ldots \ldots \ldots \ldots \ldots \ldots \ldots . \ldots 4$

$P_{c}(x) \quad$ The probability that a selected state changes according to factor $x \ldots \ldots 98$

$e \quad$ The number of aggregator samples classified in the aggregation pool... 100

v The number of possible plans that an agent generates............... 121

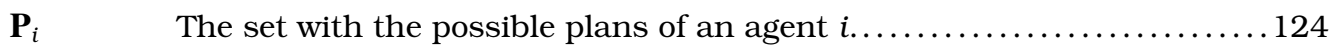

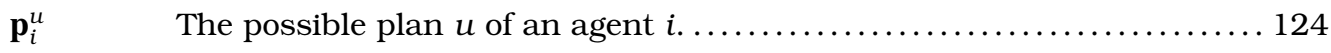

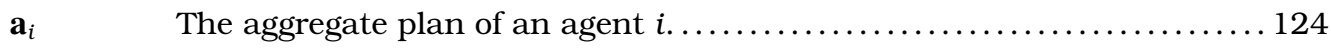

$\hat{\mathbf{a}}_{i} \quad$ An earlier aggregate plan of an agent $i \ldots \ldots \ldots \ldots \ldots \ldots \ldots \ldots \ldots \ldots \ldots \ldots \ldots$

$\mathbf{C}_{i} \quad$ The set with the combinational plans of an agent $i \ldots \ldots \ldots \ldots \ldots \ldots \ldots \ldots$ 


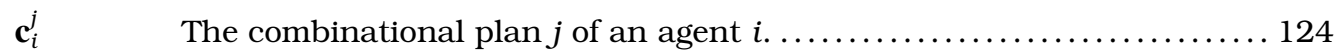

$\mathbf{c}_{i}^{\prime} \quad$ The selected combinational plan of an agent $i \ldots \ldots \ldots \ldots \ldots \ldots \ldots \ldots \ldots \ldots$

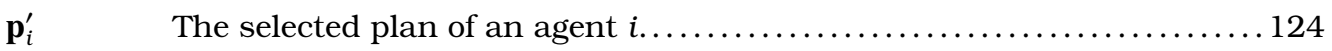

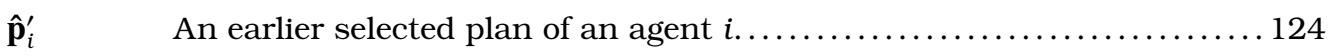

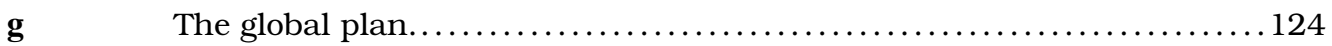

$f_{\mathrm{MD}}() \quad$ The fitness function of minimizing deviation. $\ldots \ldots \ldots \ldots \ldots \ldots \ldots \ldots \ldots$

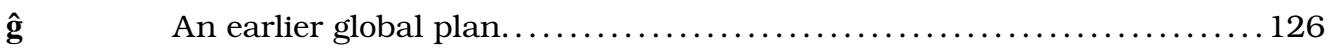

$f_{\mathrm{RD}}() \quad$ The fitness function of reversing deviation. ...................... 126

$D_{j}^{o} \quad$ The power demand of the total consumers that select the consumption mode $o$ during the day $j$ of the Olympic Peninsula Project.................. 139

$N_{j}^{o} \quad$ The number of samples (bids sent every five minutes) based on which the cumulative power demand $D_{j}^{o}$ is computed.................... 139

$N_{j} \quad$ The total number of samples (bids sent every five minutes) for the total consumers during the day $j$ of the Olympic Peninsula Project. ......... 139

$\hat{D}_{j}^{o} \quad$ The adjusted power demand of the total consumers that select the consumption mode $o$ during the day $j$ of the Olympic Peninsula Project. ........ 139

$\hat{D}_{j}^{\text {MIN }} \quad$ The minimum adjusted power demand of the total consumers during the day $j$ of the Olympic Peninsula Project.............................. 139

$\hat{D}_{j}^{\mathrm{MAx}} \quad$ The maximum adjusted power demand of the total consumers during the day $j$ of the Olympic Peninsula Project. ........................ 140 



\section{List of Figures}

1.1 An outline of this thesis. . . . . . . . . . . . . . . 8

2.1 The concept of overlay networks as a network virtualization over physical and other overlay networks. . . . . . . . . . . . . . . . 10

2.2 A simplified comparison of structured and unstructured overlay networks. 15

2.3 The four entities defined within the context of decentralized systems of overlay networks: (i) host, (ii) peer, (iii) agent and (iv) node. . . . . . . . . 18

2.4 The architecture of Protopeer (adjusted from Galuba et al. [2009]). . . . . 20

2.5 The main concepts and elements of the AgentScape middleware platform (adjusted from [Overeinder and Brazier, 2006]). . . . . . . . . . . . . 21

2.6 The main architectural components of AgentScape (adjusted from [Overeinder and Brazier, 2006]). . . . . . . . . . . . . . . . . . 22

2.7 A high-level illustration of the simulation framework architecture in AgentScape (adjusted from [Oey et al., 2010a]). . . . . . . . . . . . . . 23

3.1 The three levels of the ASMA architecture in a peer. . . . . . . . . . . 28

3.2 The executed tasks of an abstract ASMA level. . . . . . . . . . . . . 30

3.3 A two-level utilization of overlay services by distributed applications. . . . 38

4.1 An example of forming a connected undirected acyclic graph (tree). . . . 46

4.2 An example of forming a 2-ary (binary) ordered balanced complete tree. . 48

4.3 Centralized building of a binary, ordered, balanced, and complete tree by defining the tree levels incrementally. . . . . . . . . . . . . . . . 50

4.4 The AETOS middleware architecture. The architectural levels, criteria and samples realize the ones defined in ASMA as illustrated in Figure 3.1. . .

4.5 Managing the weights of agents in three levels with their views forming three layered overlay networks. . . . . . . . . . . . . . . .

4.6 Adaptations in the range of ranked nodes in the proximity view: (a) reset adaptation, (b) upgrade adaptation, (c) downgrade adaptation, (d) upgrade and downgrade adaptation. . . . . . . . . . . . . . . . . . . . 56

4.7 The selection schemes of the adaptation strategies in AETOS. . . . . . . . . 59

4.8 Convergence of connectedness for the adaptation strategies. . . . . . . . 63

4.9 Convergence of connectivity for the adaptation strategies. . . . . . . . . . 64

4.10 Convergence of fitness for the adaptation strategies. . . . . . . . . . . 65

4.11 Convergence of messages exchanged at the coordination level for the adaptation strategies. . . . . . . . . . . . . . . . . 
4.12 Visualization of the optimally organized tree topology of 1500 nodes. . . . 67

4.13 The performance of the hybrid adaptation strategies. . . . . . . . . . . 69

5.1 The DIAS middleware architecture. The architectural levels, criteria and samples realize the ones defined in ASMA as illustrated in Figure 3.1 . . 83

5.2 The aggregation memberships of DIAS. . . . . . . . . . . . 87

5.3 Classification of aggregators in the aggregation pool. . . . . . . . . . 89

5.4 The remote interactions of a bidirectional aggregation session. . . . . . . 90

5.5 Convergence of accuracy $a$ for the EXPLOITATION, UPDATE and RANDOM adaptation strategies. . . . . . . . . . . . . . . . 103

5.6 The number of outcomes for the EXPLOITATION, UPDATE and RANDOM adaptation strategies. . . . . . . . . . . . . . . . . . . . . . 104

5.7 Number of messages $\lambda$ (sessions) exchanged during the aggregation sessions for the EXPLOITATION, UPDATE and RANDOM adaptation strategies. . . . 105

5.8 The number of outcomes for the ExPLOITATION adaptation strategy using three bloom filter schemes: (i) $\mathrm{m}=16, \mathrm{k}=24$, (ii) $\mathrm{m}=14, \mathrm{k}=24$ and (iii) $\mathrm{m}=14, \mathrm{k}=6 \ldots \ldots \ldots \ldots \ldots$

5.9 Number of messages $\lambda$ (sessions) exchanged during the aggregation sessions for the EXPLOITATION adaptation strategy using three bloom filter schemes: (i) $\mathrm{m}=16, \mathrm{k}=24$, (ii) $\mathrm{m}=14, \mathrm{k}=24$ and (iii) $\mathrm{m}=14, \mathrm{k}=6$. . .

5.10 Number of messages $\lambda$ (sessions) exchanged during the aggregation sessions of DIAS for the ExPLOITATION adaptation strategy using (i) $z=10$ and (ii) $z=5 \ldots \ldots \ldots \ldots \ldots \ldots$ 108

6.1 A simplified illustration of two possible energy plans. Possible plan 'A' is a conventional one that always uses the same temperature setpoints. In contrast, possible plan ' $\mathrm{B}$ ' is generated by modifying the temperature setpoints. The power distribution of plan ' $\mathrm{B}$ ' over time is different than the one of plan 'A'. . . . . . . . . . . . . . . . . . . . . . . . . 122

6.2 The coordination algorithm of EPOS. . . . . . . . . . . . . . . 125

6.3 The planning information used by agents in decision-making. . . . . . . 126

6.4 The minimization of deviation achieved with centralized coordination, EPOS and the intended energy consumption in SETTing A. . . . . . . . . 130

6.5 The global plans of three consecutive coordination phases during which three consumption profiles are utilized. Results are computed using SETTING B. . . . . . . . . . . . . . . . . . . . . . 131

6.6 The reverse of deviation achieved with EPOS in SETTING C. . . . . . . . . 131

6.7 The effect of varying the number of possible plans $v$ that agents generate in EPOS. The data are collected using SETTING B. . . . . . . . . . . . . . . 132

6.8 The effect of varying the percentage of plan deviation from the average in EPOS. The data are collected using SETTING C. . . . . . . . . . . . . . 133

6.9 The effect of varying the number of children $k$ per agent in EPOS. The data are collected using SETTING D. . . . . . . . . . . . . .

6.10 Demand-side energy management using ALMA. Consumers select between different demand options representing levels of comfort and economy in their energy consumption. The selections are based on the aggregate demand and capacity in the Smart Power Grid. 
6.11 The actual, minimum and maximum adjusted power demand during the project year by performing selection of consumption modes.

B.1 Five beta distribution used as the input domain of the possible states. In each aggregation epoch, a random value from a given beta distribution is assigned to a possible state. . . . . . . . . . . . . . 175

B.2 Convergence of aggregates under synchronous changes for the EXPLOITATION, UPDATE and RANDOM strategies. . . . . . . . . . . . . . 176

B.3 Convergence of AVERAGE for the adaptation strategies under asynchronous changes. . . . . . . . . . . . . . . . . . 176

B.4 Convergence of summation for the adaptation strategies under asynchronous changes. . . . . . . . . . . . . . . . . 176

B.5 Convergence of MAXIMUM for the adaptation strategies under asynchronous changes. . . . . . . . . . . . . . . . . 176

C.1 The database schema of the Olympic Peninsula Project [Hammerstrom, 2007]. . . . . . . . . . . . . . . . . 178 



\section{List of Tables}

4.1 The experimental settings for the evaluation of AETOS. The bold values are

the default ones in the performed experiments. . . . . . . . . . .
4.2 Visualization of the adaptation strategies at four time points during the runtime of AETOS: (i) $20^{\text {th }}$, (ii) $35^{\text {th }}$, (iii) $50^{\text {th }}$ and (iv) $350^{\text {th }}$ epoch. . . . . . . .

4.3 Visualization of the tree topologies at three different epochs for three hybrid strategies on the (i) $245^{\text {th }}$, (ii) $255^{\text {th }}$ and (iii) $380^{\text {th }}$ epoch. . . . . . . . . . 70

4.4 Performance results of adaptation strategies for different tree topologies: (i) $k=3$, (ii) $k=5$ and (iii) $k=\operatorname{rand}(3,4,5)$. The values concern the (i) connectedness $\beta$, (ii) connectivity $\gamma$, (iii) fitness $\rho$ and (iv) the number of messages $\lambda($ coordination level). Each pair of values refers to the 30 th and 380th epoch of runtime. . . . . . . . . . . . . . . . . . 72

4.5 An overview of related decentralized mechanisms to AETOS. . . . . . . . . . 74

5.1 The experimental settings for the evaluation of DIAS. The bold values are the default ones in the performed experiments. . . . . . . . . . . . 99

5.2 An overview of related decentralized mechanisms to DIAS. . . . . . . . . . . 110

6.1 The experimental settings used for the evaluation of EPOS. . . . . . . . . . 129

6.2 The minimum adjustments of power demand relative to the actual one based on selections of occupancy modes, heating/cooling modes and their combination. The results concern the CONTROL group and the consumers with FIXED, TIME OF USE and REAL TIME PRICING contract. . . . . . . . . . . . .

The maximum adjustments of power demand relative to the actual one based on selections of occupancy modes, heating/cooling modes and their combination. The results concern the CONTROL group and the consumers with FIXED, TIME OF USE and REAL TIME PRICING contract. . . . . . . . . . . . . 



\section{List of Algorithms}

3.1 Generations of output events in an ASMA level. . . . . . . . . . . . . . . . 31

3.2 Reactions of input events in an ASMA level. . . . . . . . . . . . . . . . . . . . 31

3.3 Event generations by a gossip-based discovery level. . . . . . . . . . . . . . . . . . . 32

3.4 Event reactions by a gossip-based discovery level. . . . . . . . . . . . . . . . . . . . 32

3.5 The adapt task in a gossip-based discovery level. . . . . . . . . . . . . . . . 33

3.6 The consume task in a gossip-based discovery level. . . . . . . . . . . . 33

3.7 Event generations by the structuring level. . . . . . . . . . . . . . . . . . . . . . . . 34

3.8 Event reactions by the structuring level. . . . . . . . . . . . . . . . . . . . . . . . 35

3.9 The adapt task in the structuring level. . . . . . . . . . . . . . . 35

3.10 The consume task in the structuring level. . . . . . . . . . . . . . . 35

3.11 Event generations by the coordination level. . . . . . . . . . . . . 36

3.12 Event reactions by the coordination level. . . . . . . . . . . . . . . . . . . . . . . . . . . 37

3.13 The adapt task in the coordination level. . . . . . . . . . . . . . . . 37

3.14 The consume task in the coordination level. . . . . . . . . . . . . . 37

4.1 A centralized computation of an optimally organized tree topology with the properties introduced in Section 4.1.1. . . . . . . . . . . . . . 49

4.2 T-MAN functionality embedded in the adapt task of the structuring level in AETOS. . . . . . . . . . . . . . . . . . . . . . . . . . 55

4.3 T-MAN functionality embedded in the consume task of the structuring level in AETOS. . . . . . . . . . . . . . . . . . . . . . . . . .

5.1 The operations of an aggregation session embedded in the adapt task of the structuring level in DIAS. . . . . . . . . . . . . . . . . .

5.2 The operations of an aggregation session embedded in the consume task of the structuring level in DIAS. . . . . . . . . . . . . . . . . . . . . . . . 92

5.3 The first level of the nested mutual membership check for consistent aggregation sessions. . . . . . . . . . . . . . . . . . . . 96

5.4 The second level of the nested mutual membership check for consistent aggregation sessions. . . . . . . . . . . . . . . . . 97

6.1 The local coordination algorithm of EPOS. . . . . . . . . . . . . . . 123

A.1 The initializeCoordination subtask: Initiation of the tree coordination in AETOS performed by the consume task of the coordination level. 170

A.2 The finalizeCoordination subtask: Completion of the tree coordination in AETOS performed by the adapt task of the coordination level. . 170

A.3 The organize subtask of the coordination level in AETOS. . . . . . . . . 171

A.4 The reactions to a parent request message received in the coordination level of AETOS. . . . . . . . . . . . . . . . . . . . . . 171 
A.5 The reactions to a child request message received in the coordination level of AETOS. . . . . . . . . . . . . . . . . . . . . . . . . . 172

A.6 The reactions to an acknowledgment message received in the coordination level of AETOS. . . . . . . . . . . . . . . . . . . . . . . . 173

A.7 The reactions to a rejection message received in the coordination level of AETOS. . . . . . . . . . . . . . . . . . 173

A.8 The reactions to a removal message received in the coordination level of

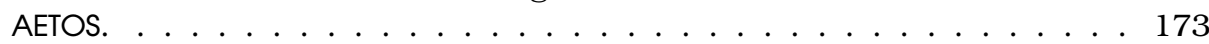




\section{Index}

A

abstraction, 3, 27

accuracy, 101

adaptation, 55

downgrade, 56

reset, 55

upgrade, 55

adaptation strategy, 34, 58, 88, 148

воттом, 59

вотTOM-UP, 59

GREEDY, 59

HUMBLE, 58

MYOPIC, 58

PRESBYOPIC, 58

TOP, 59

TOP-DOWN, 59

EXPLOITATION, 88

UPDATE, 88

AETOS, 39, 51, 127

agent, 18

AgentScape, 20, 60

simulation framework, 21, 60

aggregate, 39, 81

aggregation, 39, 81, 85

membership, 80, 86

phase, 85

pool, 88

session, 89

bidirectional, 85

consistent, 85

duplicate, 95

exploitation, 95

inconsistency, 96

unidirectional, 85

update, 95

value, 81

duplicate, 82

outdated, 82

static, 82 view, 88

aggregator, 83

exploited, 88

outdated, 88

unexploited, 88

ALMA, 135

application criteria, 38

application level, 38

application samples, 38

ASMA, 27, 51, 84

asynchronous change, 99

B

balancing, 47

behavioral science, 5

beta distribution, 98, 175

bidding, 138

bloom filter, 93

counting, 93

false negative, 94, 98

false positive, 94

simple, 93

brute force, 122

byzantine attack, 23

C

CAN, 12

candidate

children, 54

parents, 54

Chord, 12

classification, 88

clustering, 13, 53

coexistence, 9

comfort level, 135

completeness, 47

complex system, 1

component, 45

conceptual architecture, 27 
connectedness, 50

connectivity, 50

Constantine P. Cavafy, vii

consumer mode, 138

heating/cooling mode, 138

occupancy mode, 138

contract, 137

convergence, 51

coordination level, 28, 36, 51, 91

coordination phase, 123

coordination step, 123

criterion, 29

customization, 27

\section{D}

decentralized system, 2

degree-bounding, 46

Dehems, 118

demand-response program, 117

Democritus, 147

design science, 5

DIAS, 39, 82, 135

discovered samples, 29, 51, 53

discovery criteria, 29, 51

discovery level, 28, 31, 51, 52, 85

disseminator, 83

distributed hash table, 12, 33

distributed system, 2

\section{$\mathbf{E}$}

economy level, 135

Empedocles, 79

energy management

demand-side, 2, 116

automation level, 119

decentralized, 119

production-side, 2, 115

energy policy, 117

energy utilization, 120

global, 120

Epictetus, 115

EPOS, 120

F

feedback, 56

negative, 58

positive, 57

feeder, 137 fitness, 50

fitness function, 36, 126

flooding, 33

forest, 45

function-dependence, 81

G

Gnutella, 13, 17

gossiping, 13, 32, 54, 85

graph

acyclic, 45

connected, 45

undirected, 45

H

Heraclitus, 43

home automation technology, 117

host, 17

I

incentive, 117

incoming criteria, 30

incoming samples, 30

inheritance, 9

instability, 51

intended energy consumption, 122

interaction

horizontal, 29

vertical, 29

Internet of things, 10

Internet service provider, 10

Internet-scale Control System, 137

interpretivism, 5

iOverlay, 40

$\mathbf{J}$

JUNG, 62

$\mathbf{K}$

Kademlia, 12

L

load-adjustment, 116

load-shifting, 116, 143

M

MACEDON, 40

marginal price, 138

matching, 101 
message, 57, 90

acknowledgment, 57

rejection, 57

removal, 58

request, 57

middleware, 20, 27

minimum deviation, 120

mobility, 20

strong, 20

weak, 20

modularity, 3, 27

MOSAIC, 40

multicasting

application-level, 11

IP, 11

mutual membership check, 94

\section{$\mathbf{N}$}

Nikos Kazantzakis, 1

node, 18

\section{$\mathbf{0}$}

OCALA, 40

open overlay, 39

Opus, 40

ordering, 47

organizational criteria, 29, 51, 57

organized samples, 29, 51

structuring criteria, 56

outgoing criteria, 30

outgoing samples, 30, 55

overlay based services, 19

overlay network, 2,9

competitive, 16

cooperative, 16

hybrid, 17

structured, 11, 12

super-peer, 12

tree, $12,39,44$

depth, 46

height, 46

unstructured, 11

overlay service, 18

quality, 19

$\mathbf{P}$

Pastry, 12

path uniqueness, 45 peer, 17

peer sampling service, 14,52

peer-to-peer, 10, 57

peerlet, 19

plan, 121

combinational, 122

global, 122

possible, 121

selected, 122

PlanetLab, 19, 150

positivism, 4

Protopeer, 19, 60, 98

purist vs pluralist argument, 23

$\mathbf{R}$

random walk, 14, 33

ranking distance, 54

realization, 28

recursion, 9

resource-based economy, 150

reversed deviation, 121

routing-dependence, 81

\section{$\mathbf{S}$}

sample, 29

scaling factor, 54

secure hash algorithm, 12

security, 10, 22, 145, 150

self-

optimization, 36,44

organization, 41,48

service overlay network, 18

service-level agreement, 16, 19

skip list, 12

Smart Power Grid, 1

stabilization, 120

state, 84

outdated, 85

possible, 85

selected, 85

structured samples, 29

structuring criteria, 29, 51

structuring level, 28, 33, 51, 53, 85

structured samples, 51

sustainable development, 150

synchronous change, 99

synopsis diffusion, 110 
T-MAN, 54

Tapestry, 12

temperature setpoint, 121

Thales of Miletus, 27

The Olympic Peninsula Project, 137

thermostatic appliance, 120

trade-off, 70, 77, 107, 149

$\mathbf{U}$

utilization level, 38

$\mathbf{V}$

Viceroy, 12

view, 18

proximity, 54

random, 53

tree, 48

virtual power plant, 117

virtualization, 9

visualization, 62

$\mathbf{x}$

XSiena BloomFilter, 100 


\section{Samenvatting (Dutch Summary)}

Het ontwerp en beheer van gedecentraliseerde, grootschalige, netwerk vormende systemen is geen geringe uitdaging. Het is gebruikelijk om dergelijke systemen te organiseren in virtuele netwerken; de zogenaamde overlaynetwerken. Een overlaynetwerk is gepositioneerd in de applicatielaag, bovenop fysieke of andere overlaynetwerken. Overlaynetwerken implementeren complexe applicaties waarbij de organisatorische functionaliteit niet wordt gefaciliteerd door onderliggende netwerkstructuren. Deze ontwerpmethodiek heeft als nadeel dat zij een gebrek heeft aan abstractie, modulariteit en de mogelijkheid om applicaties gebaseerd op overlaynetwerken eenvoudig te kunnen herconfigureren. Dit proefschrift introduceert daarom de conceptuele architectuur ASMA (Adaptive Selforganization in a Multi-level Architecture). ASMA, de voornaamste bijdrage van dit proefschrift, is ontworpen om de zogenaamde overlayservices te kunnen ontwikkelen en biedt zo de mogelijkheid om middleware te construeren voor gedistribueerde applicaties.

Door de multi-level benadering van ASMA wordt abstractie, modulariteit en herconfigureerbaarheid verkregen. ASMA definieert derhalve drie conceptuele lagen binnen overlaynetwerken welke interactiepatronen, localisatie, structurering en coördinatie van individuele systeem entiteiten aanbieden zonder dat de aanwezigheid van een centrale beheersentiteit vereist is. De terugkoppelingsmechanismen tussen deze drie ASMA lagen verhogen stapsgewijs de kwaliteit van de aangeboden diensten. Dit proefschrift laat zien dat eenvoudige algoritmen bestaande uit slechts enkele expressies voldoende zijn om dergelijke complexe functionaliteit te realiseren. Bovendien worden twee overlayservices geïntroduceerd: (i) AETOS (de Adaptive Epidemic Tree Overlay Service) en (ii) DIAS (de Dynamic Intelligent Aggregation Service). Beide overlayservices bieden generieke mogelijkheden voor gedistribueerde systemen en verleggen hiermee technische grenzen. AETOS creëert en beheert overlaynetwerken georganiseerd in boomstructuren en voldoet aan uiteenlopende criteria gesteld aan applicaties. DIAS berekent verschillende aggregatie functies over een set van aan verandering onderhevige waarden welke gedistribueerd zijn binnen het overlaynetwerk. Van beide overlayservices wordt een experimenteel ontwerp getoond. In vergelijking met overlayservices in gerelateerde literatuur biedt ASMA een hogere abstractie, modulariteit en herconfigureerbaarheid echter tegen hogere communicatie kosten in de netwerken.

AETOS voorziet in zelforganisatie van de topologie, resulterend in een geordende, gebalanceerde en volledige boom. AETOS identificeert individuele knopen met behulp van een 'gossiping' model. De knopen, gerangschikt op basis van de applicatiecriteria, worden geclusterd op basis van hun euclidische afstand waarbij kandidaatouder en -kind knopen worden aangewezen. Deze techniek heeft een hogere kosteneffectiviteit dan bijvoorbeeld willekeurige zoektechnieken. Met behulp van 'verzoek', 'bevestigd', 'gewei- 
gerd' en 'verwijder' interactie patronen wordt er onderhandeld over verbindingen tussen deze ouders en kinderen. Verschillende boom topologieën kunnen met deze strategie worden gecreëerd; waarbij bovendien the complexe clustering en configuratie zich gedecentraliseerd afspeelt. Voor verschillende topologieën zijn experimenten uitgevoerd om overwegingen die een rol spelen bij de prestaties en herconfigureerbaarheid van netwerken inzichtelijk te maken. Deze experimenten laten bovendien zien dat AETOS inderdaad een generieke en flexibele overlayservice is voor zelforganiserende boom topologieën.

DIAS maakt aggregaties, bijvoorbeeld: gemiddelde, sommatie, maximum, etc., lokaal beschikbaar voor elke node in het overlaynetwerk. In tegenstelling tot menig andere aggregatiemethode is DIAS functie en route mechanisme onafhankelijk. Bovendien worden de aggregaties dynamisch uitgevoerd zodat wijzigingen in de invoer worden opgenomen in het resultaat. DIAS bereikt deze abstractie en flexibiliteit door gebruik te maken van zogenaamde aggregatielidmaatschappen. Een aggregatielidmaatschap bevat historische informatie over een eerder berekende aggregatie en een indicatie of dat resultaat actueel, verouderd of een duplicaat is. Dit onderscheid garandeert een accurate berekening van de aggregatiefunctie. Ook biedt dit onderscheid de mogelijkheid tot verschillende adaptatiestrategieën waarbij gekozen kan worden om gebruik te maken van verouderde of actuele resultaten. Volledige, gecentraliseerde, opslag van de aggregatielidmaatschappen is geen schaalbare oplosing. Desalniettemin bewaart DIAS de aggregatielidmaatschap in een probabilistische datastructuur: de bloom filters. Een bloom filter levert een grote opslagbesparing met als prijs de introductie van valspositieven. Een gedistribueerd mechanisme in DIAS detecteert deze vals-positieven en verzekert daarmee een accurate uitkomst in aggregatieberekeningen. Verschillende experimenten bevestigen de hoge accurate uitkomsten van DIAS in diverse opstellingen en met prestatie criteria.

De toepasbaarheid van AETOS en DIAS is bestudeerd in het domein van de Smart Power Grid. Meer specifiek, twee gedecentraliseerde, vraag gestuurde, beheersmechanismen zijn geconstrueerd op basis van de twee overlayservices: (i) EPOS, (Energy Plan Overlay Self-stabilization) en (ii) ALMA, (Adaptive Load Adjustment by Aggregation). EPOS en ALMA zijn de belangrijkste bijdrage in dit proefschrift aan het applicatiedomein van Smart Power Grids.

EPOS coördineert het energieverbruik van een groot aantal thermostaat gestuurde apparaten; zoals koelkasten en vrieskasten. EPOS zorg voor zelf-stabilisatie van het netwerk, wanneer gewenst, door de reductie van oscillatie effecten en pieken in het energieverbruik. De thermostaat gestuurde apparaten worden gecontroleerd door onderling communicerende software agenten die een geaggregeerd gegenereerd controle plan selecteren en uitvoeren zonder dat de impact direct voelbaar is voor consumenten. EPOS behaalt deze stabiliteit in energieverbruik door gebruik te maken van AETOS om de agenten te organiseren in een drielaags overlaynetwerk waarin een geaggregeerd gedecentraliseerd proces het lokale energieverbruik gereguleert. Experimentele evaluatie, op basis van synthetische data, laat zien dat een grote mate van stabilisatie bereikt wordt in verschillende scenario's.

ALMA is een aanvulling voor EPOS wanneer, in uitzonderlijke situaties zoals een storing in het netwerk of excessief energieverbruik in het energienet, een daadwerkelijke verlaging of verhoging van het verbruik noodzakelijk is. ALMA beïnvloed het geaggregeerde energieverbruik door lokaal verbruik aan te passen op basis van diverse vooraf gedefinieerde comfortniveaus van consumenten. Geaggregeerde informatie aangaande het energieverbruik kan door een DIAS overlayservice aan consumenten beschikbaar 
worden gesteld. De technische haalbaarheid van ALMA is analytisch aangetoond met behulp van data van een operationeel Smart Power Grid: het Olympic Peninsula Smart Grid Demonstration Project.

Concluderend: dit proefschrift laat zien dat de introductie van gedecentraliseerde computersystemen in nieuwe, kritieke, applicatiedomeinen, zoals het Smart Power Grid, een grote belofte is voor duurzame ontwikkeling in de maatschappij. 



\section{SIKS Dissertation Series}

\section{9}

2009-01 Rasa Jurgelenaite (RUN)

Symmetric Causal Independence Models

2009-02 Willem Robert van Hage (VU)

Evaluating Ontology-Alignment Techniques

2009-03 Hans Stol (UvT)

A Framework for Evidence-based Policy Making Using IT

2009-04 Josephine Nabukenya (RUN)

Improving the Quality of Organisational Policy Making using Collaboration Engineering

2009-05 Sietse Overbeek (RUN)

Bridging Supply and Demand for Knowledge Intensive Tasks - Based on Knowledge, Cognition, and Quality

2009-06 Muhammad Subianto (UU)

Understanding Classification

2009-07 Ronald Poppe (UT)

Discriminative Vision-Based Recovery and Recognition of Human Motion

2009-08 Volker Nannen (VU)

Evolutionary Agent-Based Policy Analysis in Dynamic Environments

2009-09 Benjamin Kanagwa (RUN)

Design, Discovery and Construction of Service-oriented Systems

2009-10 Jan Wielemaker (UVA)

Logic programming for knowledge-intensive interactive applications

2009-11 Alexander Boer (UVA)

Legal Theory, Sources of Law \& the Semantic Web

2009-12 Peter Massuthe (TUE, Humboldt-Universitaet zu Berlin)

Operating Guidelines for Services

2009-13 Steven de Jong (UM)

Fairness in Multi-Agent Systems

2009-14 Maksym Korotkiy (VU)

From ontology-enabled services to service-enabled ontologies (making ontologies work in e-science with ONTO-SOA)

2009-15 Rinke Hoekstra (UVA)

Ontology Representation - Design Patterns and Ontologies that Make Sense

2009-16 Fritz Reul (UvT)

New Architectures in Computer Chess

2009-17 Laurens van der Maaten (UvT)

Feature Extraction from Visual Data

2009-18 Fabian Groffen (CWI)

Armada, An Evolving Database System

2009-19 Valentin Robu (CWI)

Modeling Preferences, Strategic Reasoning and Collaboration in Agent-Mediated Electronic Markets

2009-20 Bob van der Vecht (UU)

Adjustable Autonomy: Controling Influences on Decision Making

2009-21 Stijn Vanderlooy (UM)

Ranking and Reliable Classification

2009-22 Pavel Serdyukov (UT)

Search For Expertise: Going beyond direct evidence
2009-23 Peter Hofgesang (VU)

Modelling Web Usage in a Changing Environment

2009-24 Annerieke Heuvelink (VUA)

Cognitive Models for Training Simulations

2009-25 Alex van Ballegooij (CWI)

RAM: Array Database Management through Relational Mapping 2009-26 Fernando Koch (UU)

An Agent-Based Model for the Development of Intelligent Mobile Services 2009-27 Christian Glahn (OU)

Contextual Support of social Engagement and Reflection on the Web

2009-28 Sander Evers (UT)

Sensor Data Management with Probabilistic Models

2009-29 Stanislav Pokraev (UT)

Model-Driven Semantic Integration of Service-Oriented Applications

2009-30 Marcin Zukowski (CWI)

Balancing vectorized query execution with bandwidth-optimized storage 2009-31 Sofiya Katrenko (UVA)

A Closer Look at Learning Relations from Text

2009-32 Rik Farenhorst (VU) and Remco de Boer (VU)

Architectural Knowledge Management: Supporting Architects and Auditors

2009-33 Khiet Truong (UT)

How Does Real Affect Affect Affect Recognition In Speech?

2009-34 Inge van de Weerd (UU)

Advancing in Software Product Management: An Incremental Method Enginee ing Approach

2009-35 Wouter Koelewijn (UL)

Privacy en Politiegegevens; Over geautomatiseerde normatieve informatie uitwisseling

2009-36 Marco Kalz (OUN)

Placement Support for Learners in Learning Networks

2009-37 Hendrik Drachsler (OUN)

Navigation Support for Learners in Informal Learning Networks

2009-38 Riina Vuorikari (OU)

Tags and self-organisation: a metadata ecology for learning resources in a multilingual context

2009-39 Christian Stahl (TUE, Humboldt-Universitaet zu Berlin)

Service Substitution - A Behavioral Approach Based on Petri Nets

2009-40 Stephan Raajjmakers (UvT)

Multinomial Language Learning: Investigations into the Geometry of Language 2009-41 Igor Berezhnyy (UvT)

Digital Analysis of Paintings

2009-42 Toine Bogers (UvT)

Recommender Systems for Social Bookmarking

2009-43 Virginia Nunes Leal Franqueira (UT)

Finding Multi-step Attacks in Computer Networks using Heuristic Search and Mobile Ambients

2009-44 Roberto Santana Tapia (UT)

Assessing Business-IT Alignment in Networked Organizations

2009-45 Jilles Vreeken (UU) 
Making Pattern Mining Useful

2009-46 Loredana Afanasiev (UvA)

Querying XML: Benchmarks and Recursion

\section{0}

2010-01 Matthijs van Leeuwen (UU)

Patterns that Matter

2010-02 Ingo Wassink (UT)

Work flows in Life Science

2010-03 Joost Geurts (CWI)

A Document Engineering Model and Processing Framework for Multimedia doc uments

2010-04 Olga Kulyk (UT)

Do You Know What I Know? Situational Awareness of Co-located Teams in Multidisplay Environments

2010-05 Claudia Hauff (UT)

Predicting the Effectiveness of Queries and Retrieval Systems

2010-06 Sander Bakkes (UvT)

Rapid Adaptation of Video Game AI

2010-07 Wim Fikkert (UT)

A Gesture interaction at a Distance

2010-08 Krzysztof Siewicz (UL)

Towards an Improved Regulatory Framework of Free Software. Protecting user freedoms in a world of software communities and eGovernments

2010-09 Hugo Kielman (UL)

A Politiele gegevensverwerking en Privacy, Naar een effectieve waarborging 2010-10 Rebecca Ong (UL)

Mobile Communication and Protection of Children

2010-11 Adriaan Ter Mors (TUD)

The world according to MARP: Multi-Agent Route Planning

2010-12 Susan van den Braak (UU)

Sensemaking software for crime analysis

2010-13 Gianluigi Folino (RUN)

High Performance Data Mining using Bio-inspired techniques

2010-14 Sander van Splunter (VU)

Automated Web Service Reconfiguration

2010-15 Lianne Bodenstaff (UT)

Managing Dependency Relations in Inter-Organizational Models

2010-16 Sicco Verwer (TUD)

Efficient Identification of Timed Automata, theory and practice

2010-17 Spyros Kotoulas (VU)

Scalable Discovery of Networked Resources: Algorithms, Infrastructure, Applications

2010-18 Charlotte Gerritsen (VU)

Caught in the Act: Investigating Crime by Agent-Based Simulation

2010-19 Henriette Cramer (UvA)

People's Responses to Autonomous and Adaptive Systems

2010-20 Ivo Swartjes (UT)

Whose Story Is It Anyway? How Improv Informs Agency and Authorship of Emergent Narrative

2010-21 Harold van Heerde (UT)

Privacy-aware data management by means of data degradation

2010-22 Michiel Hildebrand (CWI)

End-user Support for Access to Heterogeneous Linked Data

2010-23 Bas Steunebrink (UU)

The Logical Structure of Emotions

2010-24 Dmytro Tykhonov

Designing Generic and Efficient Negotiation Strategies

2010-25 Zulfiqar Ali Memon (VU)

Modelling Human-Awareness for Ambient Agents: A Human Mindreading Perspective

2010-26 Ying Zhang (CWI)

XRPC: Efficient Distributed Query Processing on Heterogeneous XQuery Engines

2010-27 Marten Voulon (UL)

Automatisch contracteren
2010-28 Arne Koopman (UU)

Characteristic Relational Patterns

2010-29 Stratos Idreos (CWI)

Database Cracking: Towards Auto-tuning Database Kernels

2010-30 Marieke van Erp (UvT)

Accessing Natural History - Discoveries in data cleaning, structuring, and retrieval

2010-31 Victor de Boer (UVA)

Ontology Enrichment from Heterogeneous Sources on the Web

2010-32 Marcel Hiel (UvT)

An Adaptive Service Oriented Architecture: Automatically solving Interoperability Problems

2010-33 Robin Aly (UT)

Modeling Representation Uncertainty in Concept-Based Multimedia Retrieval

2010-34 Teduh Dirgahayu (UT)

Interaction Design in Service Compositions

2010-35 Dolf Trieschnigg (UT)

Proof of Concept: Concept-based Biomedical Information Retrieval

2010-36 Jose Janssen (OU)

Paving the Way for Lifelong Learning; Facilitating competence development through a learning path specification

2010-37 Niels Lohmann (TUE)

Correctness of services and their composition

2010-38 Dirk Fahland (TUE)

From Scenarios to components

2010-39 Ghazanfar Farooq Siddiqui (VU)

Integrative modeling of emotions in virtual agents

2010-40 Mark van Assem (VU)

Converting and Integrating Vocabularies for the Semantic Web

2010-41 Guillaume Chaslot (UM)

Monte-Carlo Tree Search

2010-42 Sybren de Kinderen (VU)

Needs-driven service bundling in a multi-supplier setting - the computational e3-service approach

2010-43 Peter van Kranenburg (UU)

A Computational Approach to Content-Based Retrieval of Folk Song Melodies

2010-44 Pieter Bellekens (TUE)

An Approach towards Context-sensitive and User-adapted Access to Heterogeneous Data Sources, Illustrated in the Television Domain

2010-45 Vasilios Andrikopoulos (UvT)

A theory and model for the evolution of software services

2010-46 Vincent Pijpers (VU)

e3alignment: Exploring Inter-Organizational Business-ICT Alignment

2010-47 Chen Li (UT)

Mining Process Model Variants: Challenges, Techniques, Examples

2010-48 Withdrawn

2010-49 Jahn-Takeshi Saito (UM)

Solving difficult game positions

2010-50 Bouke Huurnink (UVA)

Search in Audiovisual Broadcast Archives

2010-51 Alia Khairia Amin (CWI)

Understanding and supporting information seeking tasks in multiple sources 2010-52 Peter-Paul van Maanen (VU)

Adaptive Support for Human-Computer Teams: Exploring the Use of Cognitive Models of Trust and Attention

2010-53 Edgar Mejj (UVA)

Combining Concepts and Language Models for Information Access

\section{1}

2011-01 Botond Cseke (RUN)

Variational Algorithms for Bayesian Inference in Latent Gaussian Models

2011-02 Nick Tinnemeier(UU)

Organizing Agent Organizations. Syntax and Operational Semantics of an Organization-Oriented Programming Language

2011-03 Jan Martijn van der Werf (TUE) 
Compositional Design and Verification of Component-Based Information Systems

2011-04 Hado van Hasselt (UU)

Insights in Reinforcement Learning: Formal analysis and empirical evaluation of temporal-difference learning algorithms

2011-05 Base van der Raadt (VU)

Enterprise Architecture Coming of Age - Increasing the Performance of an Emerging Discipline

2011-06 Yiwen Wang (TUE)

Semantically-Enhanced Recommendations in Cultural Heritage

2011-07 Yujia Cao (UT)

Multimodal Information Presentation for High Load Human Computer Interaction

2011-08 Nieske Vergunst (UU)

BDI-based Generation of Robust Task-Oriented Dialogues

2011-09 Tim de Jong (OU)

Contextualised Mobile Media for Learning

2011-10 Bart Bogaert (UvT)

Cloud Content Contention

2011-11 Dhaval Vyas (UT)

Designing for Awareness: An Experience-focused HCI Perspective

2011-12 Carmen Bratosin (TUE)

Grid Architecture for Distributed Process Mining

2011-13 Xiaoyu Mao (UvT)

Airport under Control. Multiagent Scheduling for Airport Ground Handling

2011-14 Milan Lovric (EUR)

Behavioral Finance and Agent-Based Artificial Markets

2011-15 Marijn Koolen (UvA)

The Meaning of Structure: the Value of Link Evidence for Information Retrieval

2011-16 Maarten Schadd (UM)

Selective Search in Games of Different Complexity

2011-17 Jiyin He (UVA)

Exploring Topic Structure: Coherence, Diversity and Relatedness

2011-18 Mark Ponsen (UM)

Strategic Decision-Making in complex games

2011-19 Ellen Rusman (OU)

The Mind's Eye on Personal Profiles

2011-20 Qing Gu (VU)

Guiding service-oriented software engineering - A view-based approach

2011-21 Linda Terlouw (TUD)

Modularization and Specification of Service-Oriented Systems

2011-22 Junte Zhang (UVA)

System Evaluation of Archival Description and Access

2011-23 Wouter Weerkamp (UVA)

Finding People and their Utterances in Social Media

2011-24 Herwin van Welbergen (UT)

Behavior Generation for Interpersonal Coordination with Virtual Humans On Specifying. Scheduling and Realizing Multimodal Virtual Human Behavior

2011-25 Syed Waqar ul Gounain Jaffry (VU)

Analysis and Validation of Models for Trust Dynamics

2011-26 Matthijs Aart Pontier (VU)

Virtual Agents for Human Communication - Emotion Regulation and InvolvementDistance Trade-Offs in Embodied Conversational Agents and Robots

2011-27 Aniel Bhulai (VU)

Dynamic website optimization through autonomous management of design patterns

2011-28 Rianne Kaptein(UVA)

Effective Focused Retrieval by Exploiting Query Context and Document Structure

2011-29 Faisal Kamiran (TUE)

Discrimination-aware Classification

2011-30 Egon van den Broek (UT)

Affective Signal Processing (ASP): Unraveling the mystery of emotions

2011-31 Ludo Waltman (EUR)

Computational and Game-Theoretic Approaches for Modeling Bounded Rational

2011-32 Nees-Jan van Eck (EUR
Methodological Advances in Bibliometric Mapping of Science

2011-33 Tom van der Weide (UU)

Arguing to Motivate Decisions

2011-34 Paolo Turrini (UU)

Strategic Reasoning in Interdependence: Logical and Game-theoretical Invest gations

2011-35 Maaike Harbers (UU)

Explaining Agent Behavior in Virtual Training

2011-36 Erik van der Spek (UU)

Experiments in serious game design: a cognitive approach

2011-37 Adriana Burlutiu (RUN)

Machine Learning for Pairwise Data, Applications for Preference Learning and Supervised Network Inference

2011-38 Nyree Lemmens (UM)

Bee-inspired Distributed Optimization

2011-39 Joost Westra (UU)

Organizing Adaptation using Agents in Serious Games

2011-40 Viktor Clerc (VU)

Architectural Knowledge Management in Global Software Development

2011-41 Luan Ibraimi (UT)

Cryptographically Enforced Distributed Data Access Control

2011-42 Michal Sindlar (UU)

Explaining Behavior through Mental State Attribution

2011-43 Henk van der Schuur (UU)

Process Improvement through Software Operation Knowledge

2011-44 Boris Reuderink (UT)

Robust Brain-Computer Interfaces

2011-45 Herman Stehouwer (UvT)

Statistical Language Models for Alternative Sequence Selection

2011-46 Beibei Hu (TUD)

Towards Contextualized Information Delivery: A Rule-based Architecture for the Domain of Mobile Police Work

2011-47 Azizi Bin Ab Aziz (VU)

Exploring Computational Models for Intelligent Support of Persons with Depression

2011-48 Mark Ter Maat (UT)

Response Selection and Turn-taking for a Sensitive Artificial Listening Agent

2011-49 Andreea Niculescu (UT)

Conversational interfaces for task-oriented spoken dialogues: design aspects influencing interaction quality

\section{2}

2012-01 Terry Kakeeto (UvT)

Relationship Marketing for SMEs in Uganda

2012-02 Muhammad Umair (VU)

Adaptivity. emotion, and Rationality in Human and Ambient Agent Model 2012-03 Adam Vanya (VU)

Supporting Architecture Evolution by Mining Software Repositories

2012-04 Jurriaan Souer (UU)

Development of Content Management System-based Web Applications

2012-05 Marijn Plomp (UU)

Maturing Interorganisational Information Systems

2012-06 Wolfgang Reinhardt (OU)

Awareness Support for Knowledge Workers in Research Networks

2012-07 Rianne van Lambalgen (VU)

When the Going Gets Tough: Exploring Agent-based Models of Human Performance under Demanding Conditions

2012-08 Gerben de Vries (UVA)

Kernel Methods for Vessel Trajectories

2012-09 Ricardo Neisse (UT)

Trust and Privacy Management Support for Context-Aware Service Platforms

2012-10 David Smits (TUE)

Towards a Generic Distributed Adaptive Hypermedia Environment

2012-11 J.C.B. Rantham Prabhakara (TUE)

Process Mining in the Large: Preprocessing, Discovery, and Diagnostic 
2012-12 Kees van der Sluijs (TUE)

Model Driven Design and Data Integration in Semantic Web Information Systems

2012-13 Suleman Shahid (UvT)

Fun and Face: Exploring non-verbal expressions of emotion during playful interactions

2012-14 Evgeny Knutov (TUE)

Generic Adaptation Framework for Unifying Adaptive Web-based Systems

2012-15 Natalie van der Wal (VU)

Social Agents. Agent-Based Modelling of Integrated Internal and Social Dynam ics of Cognitive and Affective Processes

2012-16 Fiemke Both (VU)

Helping people by understanding them-Ambient Agents supporting task execution and depression treatment

2012-17 Amal Elgammal (UvT)

Towards a Comprehensive Framework for Business Process Compliance

2012-18 Eltjo Poort (VU)

Improving Solution Architecting Practices

2012-19 Helen Schonenberg (TUE)

What's Next? Operational Support for Business Process Execution

2012-20 Ali Bahramisharif (RUN)

Covert Visual Spatial Attention, a Robust Paradigm for Brain-Computer Interfacing

2012-21 Roberto Cornacchia (TUD)

Querying Sparse Matrices for Information Retrieval

2012-22 Thijs Vis (UvT)

Intelligence, politie en veiligheidsdienst: verenigbare grootheden?

2012-23 Christian Muehl (UT)

Toward Affective Brain-Computer Interfaces: Exploring the Neurophysiology of Affect during Human Media Interaction

2012-24 Laurens van der Werff (UT)

Evaluation of Noisy Transcripts for Spoken Document Retrieval

2012-25 Silja Eckartz (UT)

Managing the Business Case Development in Inter-Organizational IT Projects: A Methodology and its Application

2012-26 Emile de Maat (UVA)

Making Sense of Legal Text

2012-27 Hayrettin Gurkok (UT)

Mind the Sheep! User Experience Evaluation \& Brain-Computer Interface Games

2012-28 Nancy Pascall (UvT)

Engendering Technology Empowering Womer

2012-29 Almer Tigelaar (UT)

Peer-to-Peer Information Retrieval

2012-30 Alina Pommeranz (TUD)

Designing Human-Centered Systems for Reflective Decision Making

2012-31 Emily Bagarukayo (RUN)

A Learning by Construction Approach for Higher Order Cognitive Skills Improve ment, Building Capacity and Infrastructure

2012-32 Wietske Visser (TUD)

Qualitative multi-criteria preference representation and reasoning

2012-33 Rory Sie (OUN)

Coalitions in Cooperation Networks (COCOON)

2012-34 Pavol Jancura (RUN)

Evolutionary analysis in PPI networks and applications

2012-35 Evert Haasdijk (VU)

Never Too Old To Learn - On-line Evolution of Controllers in Swarm- and Modular Robotics

2012-36 Denis Ssebugwawo (RUN)

Analysis and Evaluation of Collaborative Modeling Processes

2012-37 Agnes Nakakawa (RUN)
A Collaboration Process for Enterprise Architecture Creation

2012-38 Selmar Smit (VU)

Parameter Tuning and Scientific Testing in Evolutionary Algorithms

2012-39 Hassan Fatemi (UT)

Risk-aware design of value and coordination networks

2012-40 Agus Gunawan (UvT)

Information Access for SMEs in Indonesia

2012-41 Sebastian Kelle (OU)

Game Design Patterns for Learning

2012-42 Dominique Verpoorten (OU)

Reflection Amplifiers in self-regulated Learning

2012-43 Withdrawn

2012-44 Anna Tordai (VU)

On Combining Alignment Techniques

2012-45 Benedikt Kratz (UvT)

A Model and Language for Business-aware Transactions

2012-46 Simon Carter (UVA)

Exploration and Exploitation of Multilingual Data for Statistical Machine Translation

2012-47 Manos Tsagkias (UVA)

Mining Social Media: Tracking Content and Predicting Behavior

2012-48 Jorn Bakker (TUE)

Handling Abrupt Changes in Evolving Time-series Data

2012-49 Michael Kaisers (UM)

Learning against Learning - Evolutionary dynamics of reinforcement learning algorithms in strategic interactions

2012-50 Steven van Kervel (TUD)

Ontologogy driven Enterprise Information Systems Engineering

2012-51 Jeroen de Jong (TUD)

Heuristics in Dynamic Scheduling: a practical framework with a case study in elevator dispatching

\section{3}

2013-01 Viorel Milea (EUR)

News Analytics for Financial Decision Support

2013-02 Erietta Liarou (CWI)

MonetDB/DataCell: Leveraging the Column-store Database Technology for Efficient and Scalable Stream Processing

2013-03 Szymon Klarman (VU)

Reasoning with Contexts in Description Logics

2013-04 Chetan Yadati (TUD)

Coordinating autonomous planning and scheduling

2013-05 Dulce Pumareja (UT)

Groupware Requirements Evolutions Pattern

2013-06 Romulo Gonzalves (CWI)

The Data Cyclotron: Juggling Data and Queries for a Data Warehouse Audience

2013-07 Giel van Lankveld (UT)

Quantifying Individual Player Differences

2013-08 Robbert-Jan Merk (VU)

Making enemies: cognitive modeling for opponent agents in fighter pilot simulators

2013-09 Fabio Gori (RUN)

Metagenomic Data Analysis: Computational Methods and Applications

2013-10 Jeewanie Jayasinghe Arachchige (UvT)

A Unified Modeling Framework for Service Design 


\section{Publications}

Evangelos Pournaras, Martijn Warnier, and Frances M. T. Brazier. Adaptive Decentralized Aggregation. 2013a. (submitted journal).

Evangelos Pournaras, Martijn Warnier, and Frances M. T. Brazier. Peer-to-peer Aggregation for Dynamic Adjustments in Power Demand. 2013b. (submitted journal).

Evangelos Pournaras, Mark Yao, Ron Ambrosio, and Martijn Warnier. Organizational Control Reconfigurations for a Robust Smart Power Grid. In Internet of Things and Inter-cooperative Computational Technologies for Collective Intelligence, volume 460 of Studies in Computational Intelligence, chapter 8, pages 189-206. Springer-Verlag, January 2012.

Evangelos Pournaras and Shah Jahan Miah. From metaphor towards paradigm - A computing roadmap of digital ecosystems. In Proceedings of the 6th International Conference on Digital Ecosystems and Technologies, DEST 2012, pages 1-6. IEEE, June 2012.

Evangelos Pournaras, Mark Yao, and Ron Ambrosio. Dynamic composition and reconfiguration of Internet-scale control systems. In Proceedings of the 5th International Conference on Digital Ecosystems and Technologies, DEST 2011, pages 233-240. IEEE, May 2011.

Evangelos Pournaras, Martijn Warnier, and Frances M. T. Brazier. Self-Optimised Tree Overlays Using Proximity-Driven Self-Organised Agents. In Complex Intelligent Systems and their Applications, volume 41 of Springer Optimization and its Applications, chapter 7, pages 137-161. Springer New York, New York, NY, August 2010a.

Evangelos Pournaras, Martijn Warnier, and Frances M. T. Brazier. Adaptation Strategies for Self-management of Tree Overlay Networks. In Proceedings of the 11th IEEE/ACM International Conference on Grid Computing, Grid 2010, pages 401-409, Los Alamitos, CA, USA, October 2010b. IEEE.

Evangelos Pournaras, Martijn Warnier, and Frances M. T. Brazier. Local Agent-based Self-stabilisation in Global Resource Utilisation. International Journal of Autonomic Computing, 1(4):350 - 373, December 2010c.

Evangelos Pournaras, Martijn Warnier, and Frances M. T. Brazier. Towards Emergent Energy Synchronization using Agents. In Proceedings of the 1st International Workshop on Agent Technologies for Energy Systems, ATES 2010, Toronto, Canada, May 2010d. 
Evangelos Pournaras, Martijn Warnier, and Frances M. T. Brazier. Adaptive AgentBased Self-Organization for Robust Hierarchical Topologies. In Proceeding of the International Conference on Adaptive and Intelligent Systems, ICAIS 2009, pages 6976, Los Alamitos, CA, USA, September 2009a. IEEE.

Evangelos Pournaras, Martijn Warnier, and Frances M. T. Brazier. A Distributed Agentbased Approach to Stabilization of Global Resource Utilization. In Proceedings of the International Conference on Complex, Intelligent and Software Intensive Systems, CISIS 2009, pages 185-192. IEEE, March 2009b.

Evangelos Pournaras, Georgios Exarchakos, and Nick Antonopoulos. Load-driven neighbourhood reconfiguration of Gnutella overlay. Computer Communications, 31 (13):3030-3039, August 2008a.

Evangelos Pournaras, Martijn Warnier, and Frances M. T. Brazier. Using intelligent agents for self-adaptation and self-optimization of energy consumption in power networks. In Proceeding of the International Workshop on Agents for Autonomic Computing, AAC 2008, Chicago, IL, USA, June 2008b.

Evangelos Pournaras, Martijn Warnier, Frances M. T. Brazier, and Elth Ogston. Towards Adaptive Energy Plan Aggregation over a Peer-to-Peer Tree Overlay. In Proceedings of the International Workshop on Peer-to-Peer Systems, P4P2P 2008, Warwick, UK, May 2008c.

Evangelos Pournaras and Athina Lazakidou. Trust and innovativeness in virtual organisations. International Journal of Business Innovation and Research, 2(3):262-274, March 2008. 


\section{Biography}

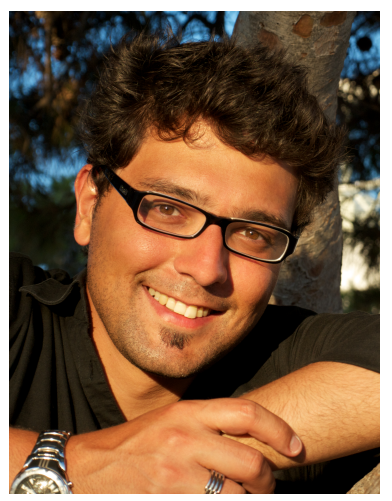

Evangelos Pournaras was born on 19th of April 1984 in Athens, Greece. He holds a BSc (2006) in Technology Education and Digital Systems from University of Piraeus in Athens. His BSc thesis focuses on distributed multimedia systems and more specifically on 3-D multiplayer games. The experimental game engine he designed and developed, JautOGL, has been a cornerstone effort on Java game development by gaining an international popularity. JautOGL was presented at the JAZOON conference and has been extensively referenced in books and online development communities. From 2003 to 2005, Evangelos worked in parallel with his BSc studies as a multimedia engineer at Largovidiaria S.A. TV Services, in Athens. In 2007, Evangelos expanded his educational background and received a MSc with Distinction in Internet Computing from University of Surrey, UK. His MSc dissertation concerns load-balancing issues in peer-to-peer systems and resulted in a journal publication.

From 2009, Evangelos became a PhD candidate of the Systems Engineering Section, Faculty of Technology, Policy and Management at Delft University of Technology in the Netherlands after moving from VU University Amsterdam where he started his PhD studies in 2007. His internship work at IBM Thomas J. Watson Research Center (2010) in the USA contributed to the Pacific Northwest Smart Grid Demonstration Project and provided him invaluable industry experience.

Evangelos has published over 14 papers in peer-reviewed international conferences and journals. He has been member in technical program committees of international conferences and workshops. The focus of his research is the self-management of decentralized systems and their application. Evangelos is currently a postdoctoral researcher in the Network Architecture and Services (NAS) group, Department of Computer Science and Engineering, Faculty of Electrical Engineering, Mathematics and Computer Science at Delft University of Technology. His postodoctoral project concerns robustness issues in the Smart Power Grid. 




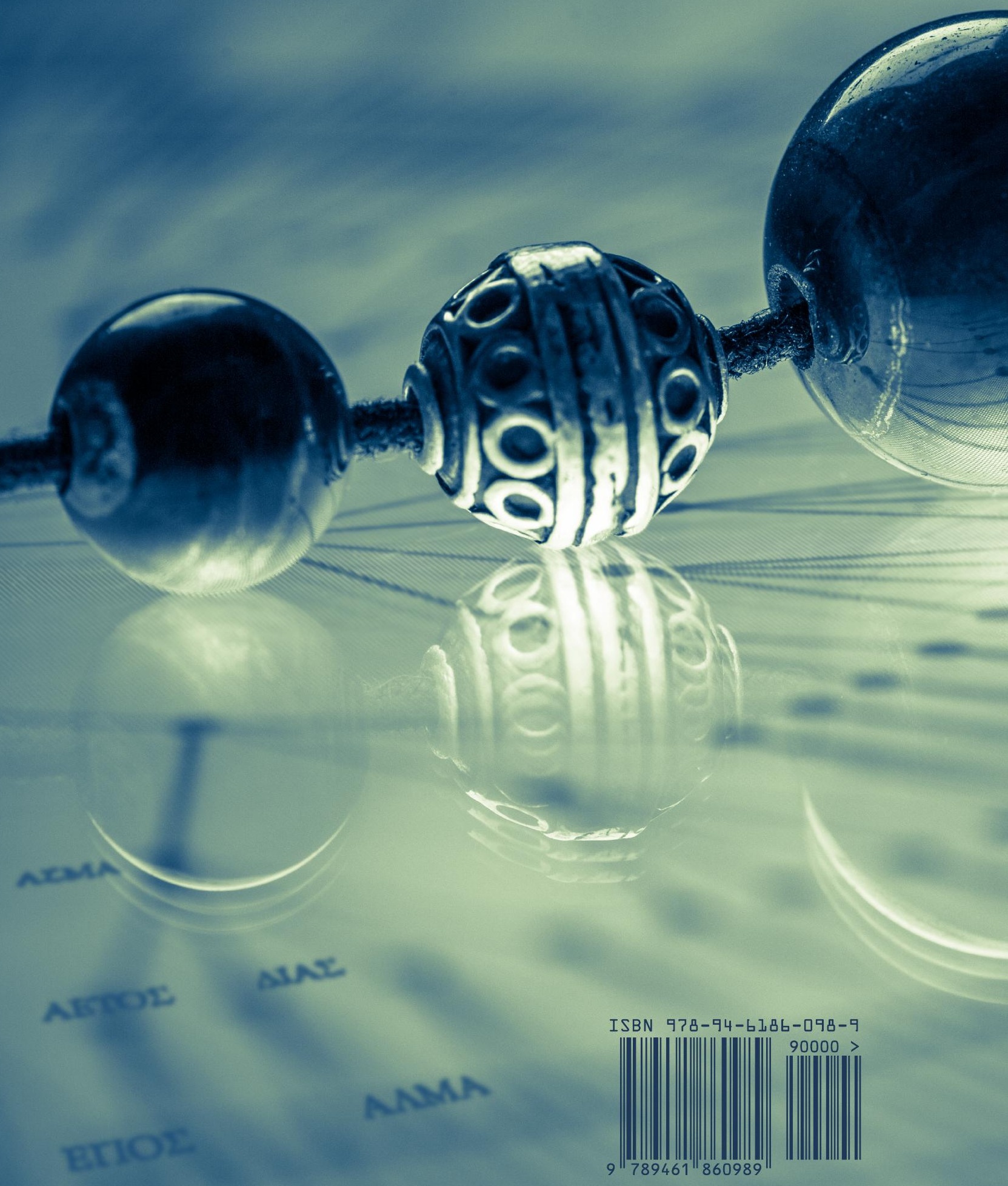

